\title{
Alternative Multimedia Regulatory Programs for Next-Generation Refineries
}

Environmental Assessment Division Argonne National Laboratory

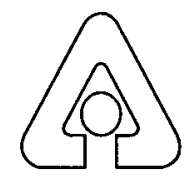

Operated by The University of Chicago, under Contract W-31-109-Eng-38, for the

United States Department of Energy 


\section{Argonne National Laboratory}

Argonne National Laboratory, with facilities in the states of Illinois and Idaho, is owned by the United States Government, and operated by the University of Chicago under the provisions of a contract with the Department of Energy.

This technical memo is a product of Argonne's Environmental Assessment Division (EAD). For information on the division's scientific and engineering activities, contact:

Director, Environmental Assessment Division

Argonne National Laboratory

Argonne, Illinois 60439-4815

Telephone (630) 252-3107

Presented in this technical memo are preliminary results of ongoing work or work that is more limited in scope and depth than that described in formal reports issued by the EAD.

Publishing support services were provided by Argonne's Information and Publishing Division (for more information, see IPD's home page: http://www.ipd.anl.gov/).

\section{Disclaimer}

This report was prepared as an account of work sponsored by an agency of the United States Government. Neither the United States Government nor any agency thereof, nor The University of Chicago, nor any of their employees or officers, makes any warranty, express or implied, or assumes any legal liability or responsibility for the accuracy, completeness, or usefulness of any information, apparatus, product, or process disclosed, or represents that its use would not infringe privately owned rights. Reference herein to any specific commercial product, process, or service by trade name, trademark, manufacturer, or otherwise does not necessarily constitute or imply its endorsement, recommendation, or favoring by the United States Government or any agency thereof. The views and opinions of document authors expressed herein do not necessarily state or reflect those of the United States Government or any agency thereof, Argonne National Laboratory, or The University of Chicago. 


\section{Alternative Multimedia Regulatory Programs for Next-Generation Refineries}

by D. Elcock, J. Gasper, R. Arguero, ${ }^{\star}$ and D. Emerson*

Environmental Assessment Division,

Argonne National Laboratory, 9700 South Cass Avenue, Argonne, Illinois 60439

February 2000

Work sponsored by the U.S. Department of Energy, Office of Policy and Office of Fossil Energy, and U.S. Environmental Protection Agency, Office of Air and Radiation, under Environmental Technology Initiative Project DW89937330-01-0

* Arguero and Emerson are affiliated with Analytical Services, Inc., Columbia, Maryland. 
This report is printed on recycled paper. 


\section{Contents}

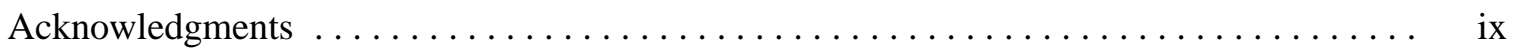

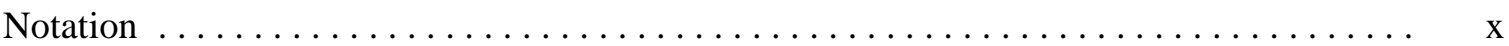

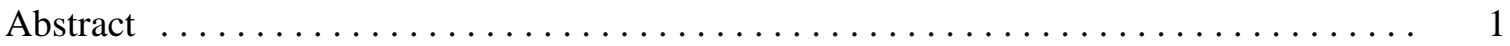

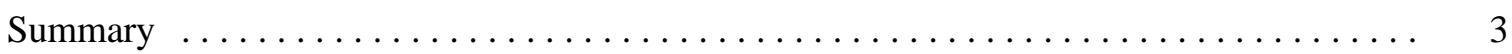

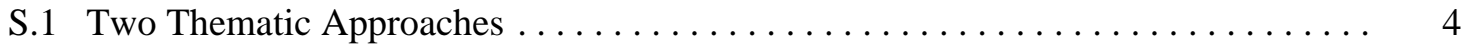

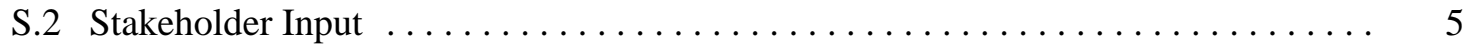

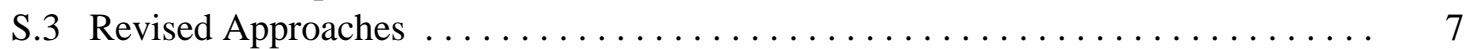

S.3.1 Revised RBB . . . . . . . . . . . . . . . . . . . . . . . 7

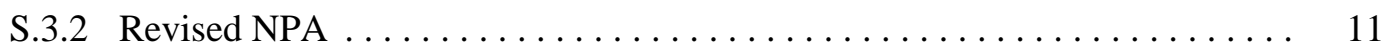

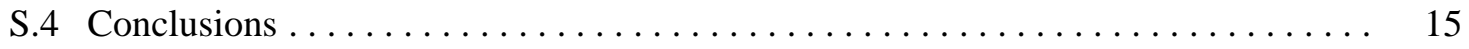

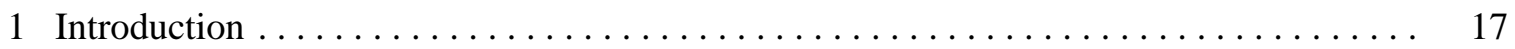

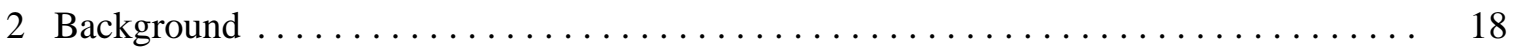

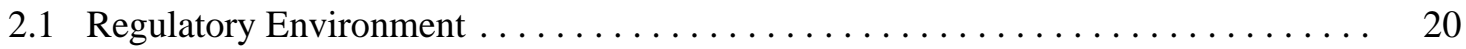

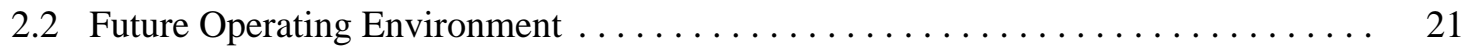

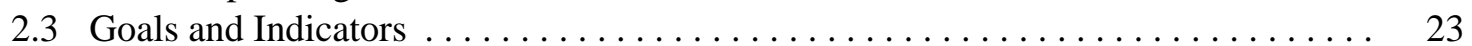

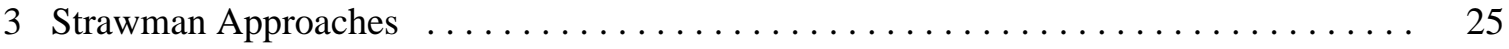

3.1 Methodology for Developing Strawman Approaches $\ldots \ldots \ldots \ldots \ldots \ldots \ldots \ldots$

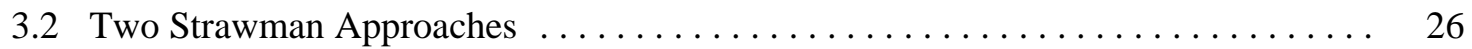

3.2.1 Elements Common to Both the RBB and the NPA Strawman

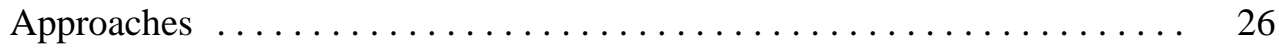

3.2.2 Elements Unique to the RBB Strawman Approach $\ldots \ldots \ldots \ldots \ldots \ldots .27$

3.2.3 Elements Unique to the NPA Strawman Approach $\ldots \ldots \ldots \ldots \ldots \ldots .28$

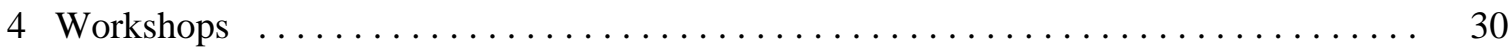

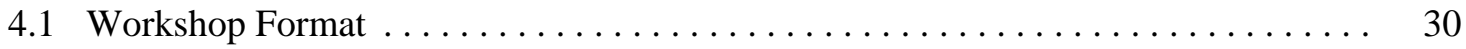

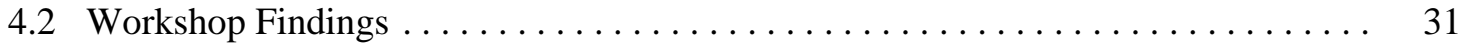

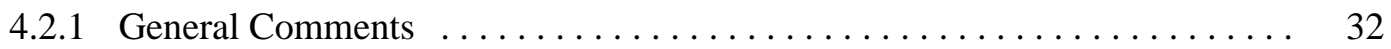

4.2.1.1 Current Regulatory Approaches Need Improvement . . . . . . . . 32

4.2.1.2 Meaningful Public Participation Is Important

throughout the Process $\ldots \ldots \ldots \ldots \ldots \ldots \ldots \ldots \ldots \ldots \ldots$ 


\section{Contents (Cont.)}

4.2.1.3 Environmental and Economic Goals Are Equally Important . . . . . 33

4.2.1.4 Approaches Need Good Performance Indicators and Measures of Success . . . . . . . . . . . . . . . . . 34

4.2.1.5 Approaches Need More Details and Specifications . . . . . . . . . . 34

4.2.1.6 Implementation Issues Must Be Addressed . . . . . . . . . . . . 34

4.2.1.7 The Boundaries Set by the Guidelines and Principles May Limit the Success of the Approaches .............. 35

4.2.1.8 Three-Component Structure Is Appropriate . . . . . . . . . 35

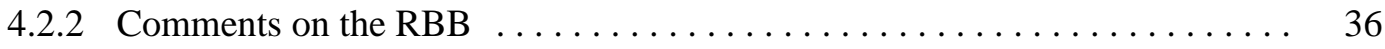

4.2.2.1 Establish Baseline ....................... 36

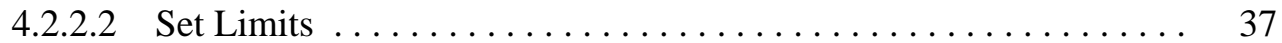

4.2.2.3 Assure Compliance ........................... 39

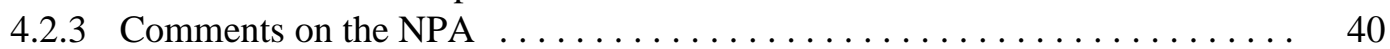

4.2.3.1 Establish Baseline . . . . . . . . . . . . . . . . . . 40

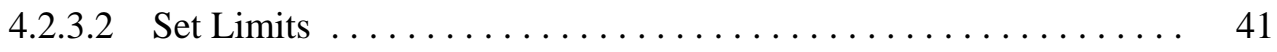

4.2.3.3 Assure Compliance ..................... 42

4.3 Using the Findings to Revise the Approaches $\ldots \ldots \ldots \ldots \ldots \ldots \ldots \ldots \ldots$

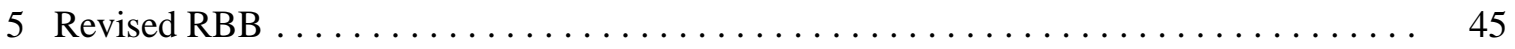

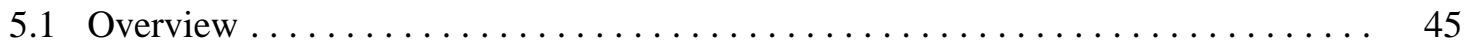

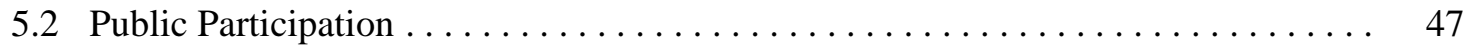

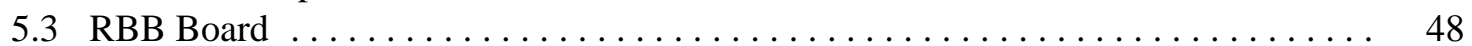

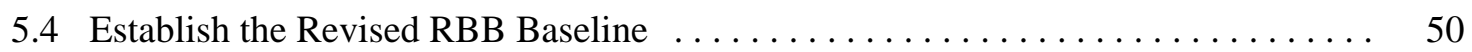

5.4 .1 Develop Inventory of Refinery Releases $\ldots \ldots \ldots \ldots \ldots \ldots \ldots \ldots \ldots . \ldots \ldots$

5.4 .2 Characterize Environment Affected by Releases $\ldots \ldots \ldots \ldots \ldots \ldots \ldots 2$

5.4 .3 Characterize Pollutants of Potential Concern ................. 54

5.4.4 Screen and Prioritize Pollutants for Setting Release Limits . . . . . . . . . . 55

5.4.4.1 California Air Toxics Program ................... 56

5.4.4.2 Minimal Risk Levels for Hazardous Substances . . . . . . . . . . 56

5.4 .4 .3 Sector Facility Indexing Project $\ldots \ldots \ldots \ldots \ldots \ldots \ldots \ldots \ldots$

5.4.4.4 Risk Screening Environmental Indicators Project . . . . . . . . . 56

5.4 .5 Document and Communicate Results . . . . . . . . . . . . . . . . 58

5.5 Set Risk-Based Release Limits in the Revised RBB . . . . . . . . . . . . . . 59

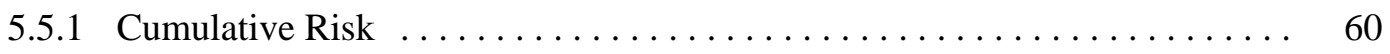

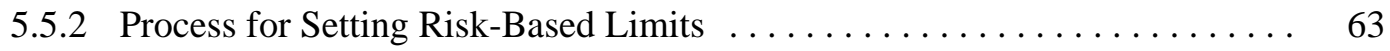

5.5.2.1 Determine Acceptable Risk . . . . . . . . . . . . . . . . 64

5.5.2.2 Calculate Initial Estimate of Total Cumulative Risk . . . . . . . . 65

5.5.2.3 Establish Initial Pollutant-Specific Risk-Based Release Limits . . . 66

5.5.2.4 Develop Alternative Risk-Based Release Limits . . . . . . . . . 67

5.5.2.5 Document and Communicate Results .............. 68

5.5.2.6 Periodically Reexamine Release Limits ............... 68

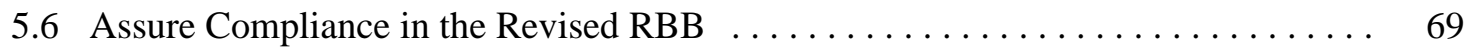

5.6.1 Compliance Assurance Measures $\ldots \ldots \ldots \ldots \ldots \ldots \ldots \ldots \ldots \ldots$ 


\section{Contents (Cont.)}

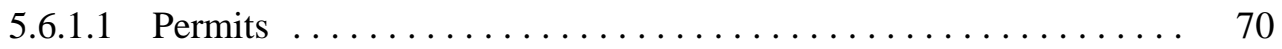

5.6.1.2 Incentives and Disincentives $\ldots \ldots \ldots \ldots \ldots \ldots \ldots \ldots \ldots \ldots$

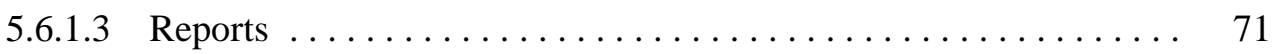

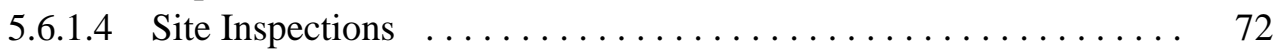

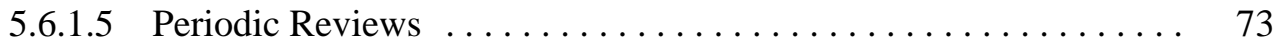

5.6.2 Preference for Monitored Exposure-Point Data $\ldots \ldots \ldots \ldots \ldots \ldots \ldots$

5.6.2.1 Accounting for Uncertainty in Risk-Based



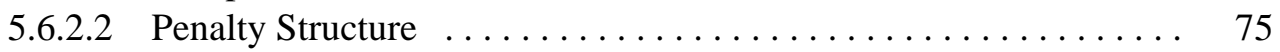

5.6.2.3 Performance-Based Measurement Systems . . . . . . . . . . . 75

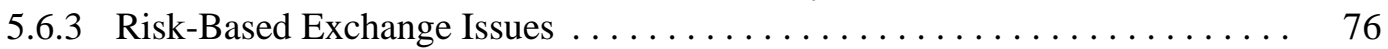

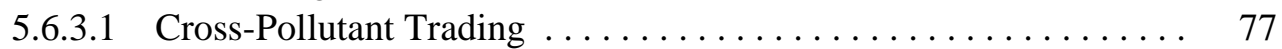

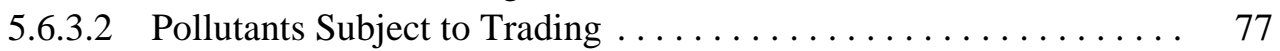

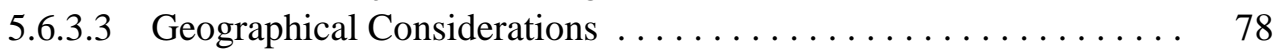

5.6.3.4 Trading as a Purely Economic Incentive $\ldots \ldots \ldots \ldots \ldots \ldots . \ldots \ldots$

5.6.3.5 Environmental Considerations $\ldots \ldots \ldots \ldots \ldots \ldots \ldots \ldots \ldots$

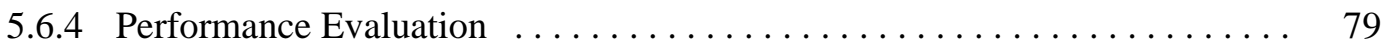

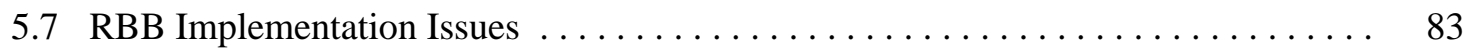

5.7.1 Issues Addressed by Ongoing Improvements in Science, Technology,

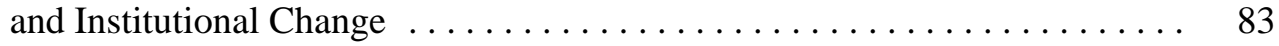

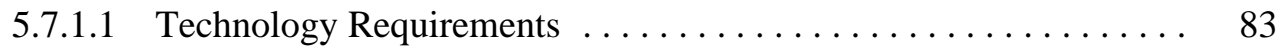

5.7.1.2 Setting Risk-Based Limits $\ldots \ldots \ldots \ldots \ldots \ldots \ldots \ldots \ldots \ldots$

5.7.1.3 Institutional Impediments to Multimedia Approaches . . . . . . . . . 84

5.7.1.4 Resource Requirements ...................... 84

5.7.1.5 Nontechnical Issues $\ldots \ldots \ldots \ldots \ldots \ldots \ldots \ldots \ldots \ldots \ldots . \ldots \ldots$

5.7 .2 Issues Addressed by Legislation $\ldots \ldots \ldots \ldots \ldots \ldots \ldots \ldots \ldots \ldots$

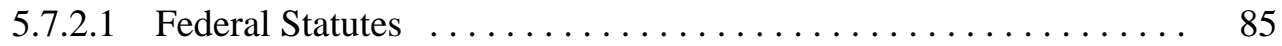

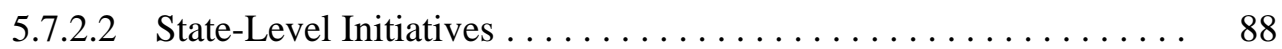

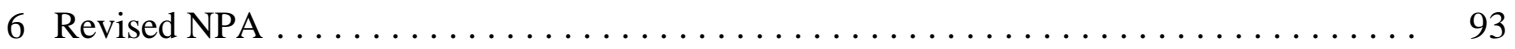

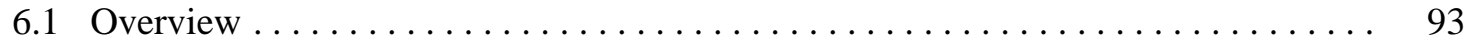

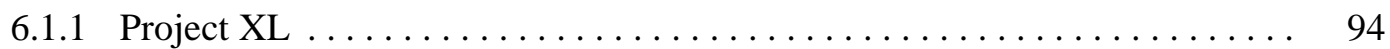

6.1.2 The Netherlands Covenants Program $\ldots \ldots \ldots \ldots \ldots \ldots \ldots \ldots \ldots . \ldots \ldots$

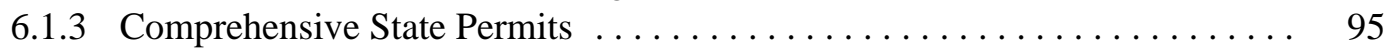

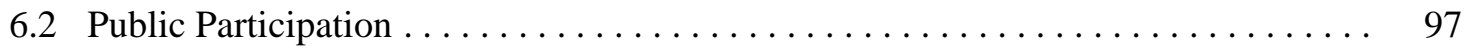

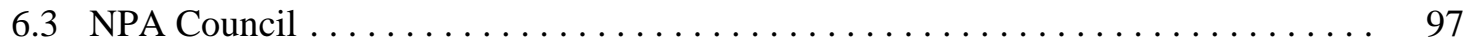

6.4 Establish Goals for the Revised NPA $\ldots \ldots \ldots \ldots \ldots \ldots \ldots \ldots \ldots \ldots \ldots \ldots$

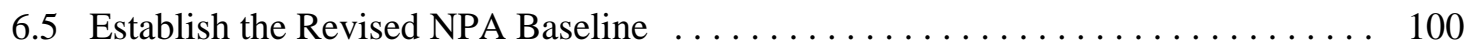

6.5.1 Develop Inventory of Refinery Releases $\ldots \ldots \ldots \ldots \ldots \ldots \ldots \ldots \ldots$

6.5.2 Develop Inventory of Environmental Management Costs . . . . . . . . 102

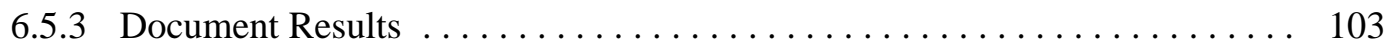

6.5.4 Prioritize Pollutants for Setting Release Limits . . . . . . . . . . . . . . . . . . 104

6.6 Set Risk-Based Release Limits in the Revised NPA . . . . . . . . . . . . . . . . . . 104 


\section{Contents (Cont.)}

6.6.1 Background on Negotiations, Covenants, and Permits . . . . . . . . . . 105

6.6.1.1 Negotiated Rulemakings ... . . . . . . . . . . . . . . . . . 105

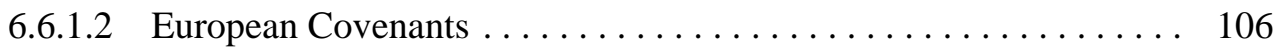

6.6.1.3 Technology Compacts ....................... 108

6.6.1.4 Japanese Environmental Agreements . . . . . . . . . . . . . . . . 108

6.6.1.5 Multimedia, Facilitywide, Integrated Permits . . . . . . . . . . 108

6.6 .2 Factors to Consider . . . . . . . . . . . . . . . . . . . . . . . . . . . . . 110

6.6.2.1 Public Participation ..................... 110

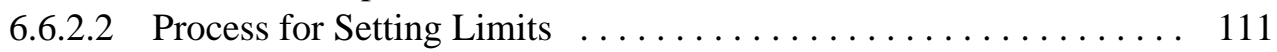

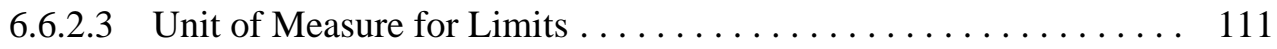

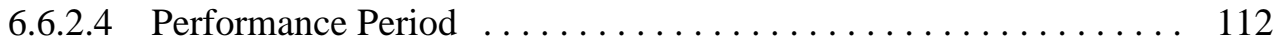

6.6.2.5 Format and Content of Agreement . . . . . . . . . . . . . . . 112

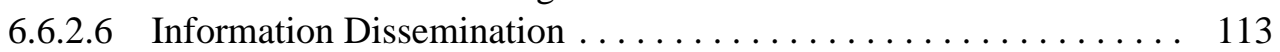

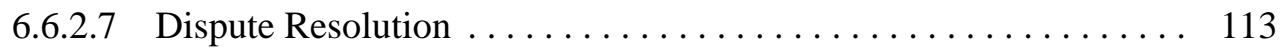

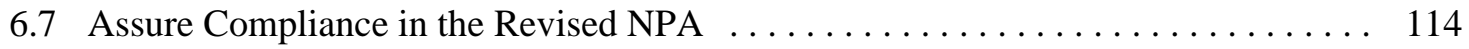

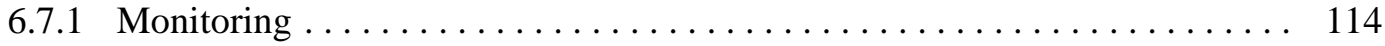

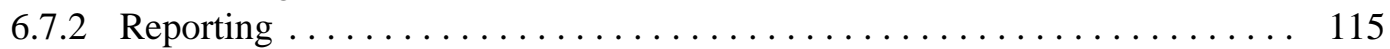

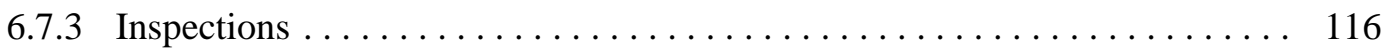

6.7 .4 Incentives . . . . . . . . . . . . . . . . . . . . . . . 117

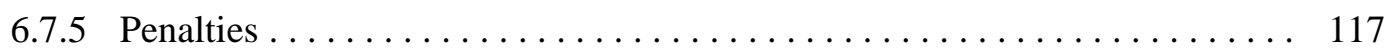

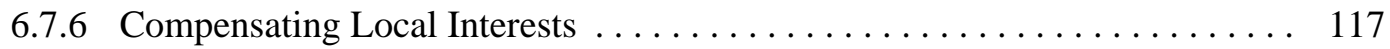

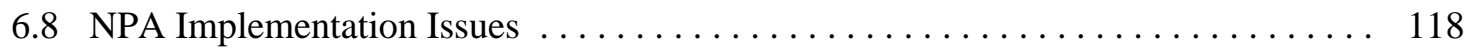

6.8.1 Resource Requirements .......................... 118

6.8.1.1 Financial Resources ... . . . . . . . . . . . . . . . . . . . . 118

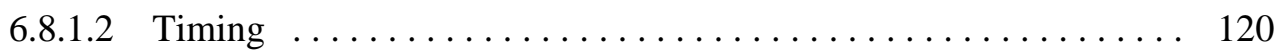

6.8 .2 Jurisdictional Issues . . . . . . . . . . . . . . . . . . 120

6.8.3 Role of National Environmental Groups . . . . . . . . . . . . . . . . . . . . 121

6.8.4 Obtaining Buy-in for New Ideas . . . . . . . . . . . . . . . . . . . 121

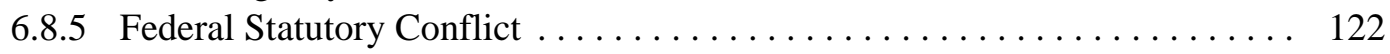

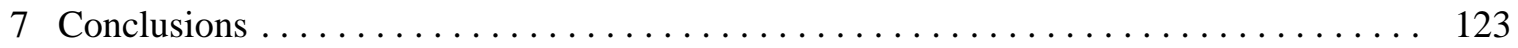

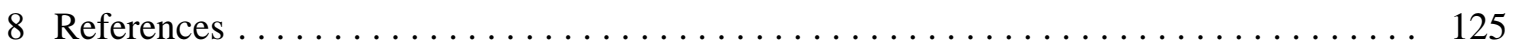

\section{Appendixes}

Appendix A: Recent Regulatory Reform Efforts $\ldots \ldots \ldots \ldots \ldots \ldots \ldots \ldots \ldots \ldots \ldots$

Appendix B: Guidelines and Principles for Developing Alternative

Regulatory Approaches . . . . . . . . . . . . . . . . . . . 135

Appendix C: Environmental Laws and Regulations Affecting Petroleum Refineries .... 137 


\section{Appendixes (Cont.)}

Appendix D: Goals and Indicators for Regulatory Options $\ldots \ldots \ldots \ldots \ldots \ldots \ldots \ldots$

Appendix E: $\quad$ Summary of Workshop Comments $\ldots \ldots \ldots \ldots \ldots \ldots \ldots \ldots \ldots \ldots .143$

Appendix F: Studies and Efforts That Support the Concepts and Provisions

of the Risk-Based Bubble ... . . . . . . . . . . . . . . . . . . . 149

Appendix G: Summary of Approaches to Legislative Change $\ldots \ldots \ldots \ldots \ldots \ldots \ldots \ldots$

Appendix H: Studies and Efforts That Support the Concepts and Provisions

of the Negotiated Performance Agreement . . . . . . . . . . . . . . . . 157

Appendix I: $\quad$ XL Projects Relevant to the Revised Negotiated

Performance Agreement $\ldots \ldots \ldots \ldots \ldots \ldots \ldots \ldots \ldots \ldots \ldots \ldots \ldots \ldots \ldots$

\section{Figures}

$1 \quad$ Regulatory Alternative Development Process $\ldots \ldots \ldots \ldots \ldots \ldots \ldots \ldots \ldots \ldots$

2 Trends in American Petroleum Institute Gravity, 1984-1998 . . . . . . . . . . . . . 22

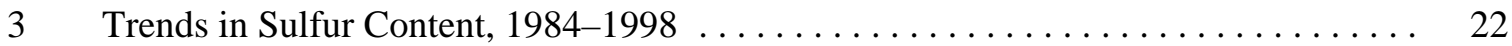

$4 \quad$ Worldwide Trends in Refinery Product Demand $\ldots \ldots \ldots \ldots \ldots \ldots \ldots \ldots \ldots$

$5 \quad$ Three-Component Regulatory Approach Structure $\ldots \ldots \ldots \ldots \ldots \ldots \ldots \ldots \ldots$

\section{Tables}

$1 \quad$ Key Elements of Current Regulatory Program $\ldots \ldots \ldots \ldots \ldots \ldots \ldots \ldots \ldots \ldots$

2 Key Elements of the Risk-Based Bubble Strawman Approach $\ldots \ldots \ldots \ldots \ldots \ldots .28$

3 Key Elements of the Negotiated Performance Agreement Strawman Approach . . . 29

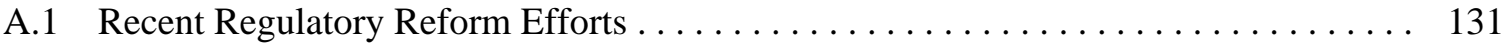

B.1 Guidelines for Developing Alternative Regulatory Approaches . . . . . . . . . . . . 135

B.2 Principles for Developing Alternative Regulatory Approaches $\ldots \ldots \ldots \ldots \ldots \ldots 136$

C.1 Environmental Laws and Regulations Affecting Petroleum Refineries . . . . . . . 137 


\section{Tables (Cont.)}

F.1 Studies and Efforts That Support the Concepts and Provisions of the Risk-Based Bubble . . . . . . . . . . . . . . . . . . . . . . . . . . . . . . . . . 149

G.1 Summary of Approaches to Legislative Change $\ldots \ldots \ldots \ldots \ldots \ldots \ldots \ldots \ldots \ldots$

H.1 Studies and Efforts That Support the Concepts and Provisions of the Negotiated Performance Agreement . . . . . . . . . . . . . . . . . . 157

I.1 XL Projects Relevant to the Revised Negotiated Performance Agreement . . . . . . . . 161 


\section{Acknowledgments}

The authors wish to thank the sponsors of this effort, including project director David O. Moses in the U.S. Department of Energy (DOE), Office of Policy; Arthur Hartstein in the DOE Office of Fossil Energy; James Durham and Doris Maxwell in the U.S. Environmental Protection Agency (EPA), Office of Air and Radiation; and Steven H. Souders in the EPA Office of Solid Waste and Emergency Response. The authors extend their special appreciation to the representatives of potentially affected parties for the valuable comments they provided during the seven workshops that were held to solicit their views on the approaches as they were being developed. 


\section{Notation}

$\begin{array}{ll}\text { ADR } & \text { alternative dispute resolution } \\ \text { APA } & \text { Administrative Procedures Act } \\ \text { API } & \text { American Petroleum Institute } \\ \text { ATSDR } & \text { Agency for Toxic Substances and Disease Registry } \\ \text { BACT } & \text { best available control technology } \\ \text { CAA } & \text { Clean Air Act } \\ \text { CAAA } & \text { Clean Air Act Amendments of 1990 } \\ \text { CARE } & \text { Clean Air Responsibility Enterprise } \\ \text { CEP } & \text { company environmental plan } \\ \text { CO } & \text { carbon monoxide } \\ \text { CO } & \text { carbon dioxide } \\ \text { CREAM } & \text { Cumulative Risk Estimating and Assessment Model } \\ \text { CWA } & \text { Clean Water Act } \\ \text { DEQ } & \text { Department of Environmental Quality } \\ \text { DOE } & \text { U.S. Department of Energy } \\ \text { ECOS } & \text { Environmental Council of the States } \\ \text { EPA } & \text { U.S. Environmental Protection Agency } \\ \text { ETI } & \text { U.S. Environmental Technology Initiative } \\ \text { FWP } & \text { facilitywide permit } \\ \text { GAO } & \text { U.S. General Accounting Office } \\ \text { GIS } & \text { geographic information system } \\ \text { GPRA } & \text { Government Performance and Results Act } \\ \text { HEAST } & \text { Health Effects Assessment Summary Table } \\ \text { HQ } & \text { hazard quotient } \\ \text { IRIS } & \text { Integrated Risk Information System } \\ \text { IRP } & \text { Integrated Risk Project } \\ \text { ISO } & \text { International Organization for Standardization } \\ \text { MACT } & \text { maximum achievable control technology } \\ \text { MIR } & \text { maximum individual risk } \\ \text { MPCA } & \text { Minnesota Pollution Control Agency } \\ \text { MRL } & \text { minimum risk level } \\ \text { NEPP } & \text { National Environmental Policy Plan } \\ \text { NESCAUM } & \text { Northeast States for Coordinated Air Use Management } \\ \text { NESHAP } & \text { National Emission Standards for Hazardous Air Pollutants } \\ \text { NHEXAS } & \text { National Human Exposure Assessment Survey } \\ \text { NJDEP } & \text { New Jersey Department of Environmental Protection } \\ \text { NOx } & \text { nitrogen oxides } \\ \text { NPA } & \text { Negotiated Performance Agreement } \\ \text { OAQPS } & \text { Office of Air Quality Planning and Standards } \\ \text { OECA } & \text { Office of Enforcement and Compliance Assistance } \\ & \end{array}$




\section{Notation (Cont.)}

OPPPC Office of Pollution Prevention and Permit Coordination (NJDEP)

OPPT

PAL Office of Prevention, Pesticides, and Toxic Substances (EPA)

PBMS

PPA

RBB plantwide applicability limit

RCRA

Rfc

Rfd

RFO

RSEI

performance-based measurement system

Pollution Prevention Alliance

risk-based bubble

SAB

Resource Conservation and Recovery Act

SEP

SFIP

$\mathrm{SO}_{2}$ reference concentration

reference dose

regulatory flexibility order

risk screening environmental indicator

Science Advisory Board

supplemental environmental project

Sector Facility Indexing Project

TNRCC Texas Natural Resources and Conservation Commission

TRI Toxic Release Inventory

TRIM total risk integrated methodology

TRRP Texas Risk Reduction Program 
A 


\section{Abstract}

Recently, many industrial, regulatory, and community leaders have expressed concern that the current environmental regulatory structure disregards multimedia environmental impacts, provides few incentives to develop and use new technologies, and fails to consider site-specific conditions. For the U.S. petroleum refining industry, faced with the need to produce higher-quality fuels from poorer-quality feedstocks, such criticisms are expected to increase. This report offers two alternative environmental regulatory programs to apply to existing petroleum refineries when they operate in the future. These alternative approaches are multimedia in scope, provide for new technology development and use, and allow flexibility in the means for meeting environmental goals. They have been reviewed and critiqued by various stakeholders, including industry representatives, regulators, and local and national community and environmental organizations. 
A 


\section{Summary}

The 25-year-old "command-and-control" environmental regulatory structure in the United States has resulted in significant environmental improvements. Recently, however, its limitations (e.g., rigid application regardless of site-specific conditions, disregard of cross-media and multimedia impacts, limited incentives for new technology development and use) have become increasingly apparent. New regulatory approaches that recognize current and anticipated economic constraints, new knowledge of environmental processes and impacts, and the benefits of new technologies are needed. Such approaches could be especially important for the U.S. petroleum refining industry. This industry operates under thin profit margins, releases chemicals that can produce adverse health and environmental impacts, and must meet the technological challenges of producing more highly refined fuels from poorer quality feedstocks.

Under a grant from the Environmental Technology Initiative (ETI), Argonne National Laboratory and its subcontractor, Analytical Services, Inc. — together, the "project team" developed two alternative environmental regulatory programs for next-generation petroleum refineries. (In this report, "next-generation refineries" refers to the refineries of today as they operate in the next 20 or more years rather than to fully reengineered future refineries.) The objective of the ETI refinery project was to develop future-oriented regulatory programs for nextgeneration refineries that will expand the use of innovative technologies, encourage pollution prevention, demonstrate environmental responsibility, and maintain refinery economic performance.

The ETI refinery project differs from other regulatory reform efforts in several ways. For example, it recognizes that the changing operational characteristics of future refineries and the environmental impacts associated with these changes will require fundamentally different programs. Rather than suggesting targeted, short-term modifications to existing media-specific command-and-control regulations, the ETI project suggests the use of new approaches that are broader and more flexible. It addresses cross-media and multimedia impacts. It recognizes that giving refineries flexibility in meeting environmental protection goals can stimulate new technology development and use. Unlike most U.S. Environmental Protection Agency (EPA) reinvention efforts, which seek results in 12 to18 months, this ETI effort assumes a time frame of 20 years or more. It also assumes that existing laws and regulations can be changed.

An iterative and interactive process was used by the project team to develop the alternative approaches. Information and stakeholder input were integrated to provide for constant revision and improvement. First, guidelines and principles were established to bound the study and set parameters for developing the approaches. (For example, the approaches pertain to refinery operations and do not include exploration, production, or product use.) Next, existing and projected environmental laws and regulations affecting petroleum refineries were examined to identify areas needing change. Then, to understand future challenges and opportunities, the projected refinery operating environment was described in terms of feedstock, product, 
technology, and economics. Finally several goals and indicators for assessing and comparing the alternatives were identified.

On the basis of this background information, more than 60 options that could efficiently and effectively protect human health and the environment were identified. These options ranged from fundamental changes in program philosophy to procedural improvements. After the options were evaluated against the goals and indicators, many of them were integrated into two separate thematic paradigms: a risk-based paradigm and a goal-based paradigm. Each paradigm was then developed into a draft, or strawman, approach consisting of three structural components: (1) establish a baseline, (2) set residual release limits, and (3) assure compliance. Both approaches share this common structure. Some of the elements within the components are common to both approaches, and some differ.

Elements common to both approaches include the following:

- Establish the baseline. In establishing the baseline, the refinery and the regulator jointly identify residuals for which release limits must be established.

- Set residual release limits. The refinery and the regulator jointly specify release limits on a facilitywide rather than a source-specific basis. A facilitywide permit documents the release limits.

- Assure compliance. Incentives provide the basis for assuring compliance, and flexibility in the compliance method is encouraged. Penalties apply if releases exceed the limits, and reporting requirements are streamlined relative to current practices.

\section{S.1 Two Thematic Approaches}

Thematically, the approaches differ. In the risk-based approach - the Risk-Based Bubble (RBB) - limits are set on the basis of risk, and releases can be traded across environmental media and pollutants. In the goal-based program - the Negotiated Performance Agreement (NPA) - release limits, which require specific reductions from current requirements, are negotiated.

Elements unique to the RBB, identified by component, include the following:

- Establish the baseline. In addition to identifying environmental releases, the RBB baseline also characterizes site-specific environmental conditions and information on receptors surrounding the refinery for use in setting the risk-based limits.

- Set residual release limits. Jointly, the refinery and the regulator set release limits on the basis of risk to public health and the environment. The process for setting limits starts with establishing the residuals of concern on the basis of toxicity or other known health or environmental effects. Then the site-specific receptors and conditions identified in the baseline are considered to establish acceptable cumulative health and ecological risk levels. The process uses risk models to establish pollutant-specific release limits, which 
are designed to keep risk within acceptable levels. The regulator and refinery reexamine the limits periodically to incorporate new information or changed conditions.

- Assure compliance. Refiners can select or develop their own approaches for meeting the limits and can trade releases across media and pollutants on the basis of risk. Linking of electronic monitoring results with risk models will facilitate trading and help assure compliance.

Elements unique to the NPA include the following:

- Establish the baseline. The NPA baseline inventories both current releases and environmental management costs. The baseline provides a starting point for identifying more cost-effective environmental management options.

- Set residual release limits. Limits are negotiated to achieve pollutant- and media-specific reductions, expressed as percentage or actual reductions, from the baseline.

- Assure compliance. The environmental performance agreement specifies the release limits and compliance assurance requirements, which remain in force for a specified period of time. Progress in reducing releases is measured against the baseline. If limits or reduction goals are not met, affected interests (e.g., local citizens) may be compensated.

\section{S.2 Stakeholder Input}

To meet user needs, it was deemed essential to incorporate feedback and comments in developing the approaches. Thus, before advancing details of the two approaches, several workshops were conducted to exchange information and obtain input for refining and improving the two approaches. To promote candid dialogue, the workshops were small and lasted for one day, and each followed the same format and was attended by representatives from a single interest group. Represented interest groups included petroleum refiners and trade associations, EPA headquarters offices, Texas and Louisiana state regulatory representatives, Texas and Louisiana citizens groups, national environmental groups, Mid-Atlantic state regulatory representatives, and Mid-Atlantic citizens groups. In each workshop, a facilitator solicited comments and feedback on the three-component structure and the two strawman approaches. Participants provided constructive criticism, candid observations, and thoughtful suggestions for improving the approaches. Most agreed that the current environmental regulatory system needs to be improved. However, opinions on the degree and nature of required change varied, and participants stressed that they needed to see more details before they could endorse or oppose specific elements or approaches. Other commonly expressed comments include the following:

- Meaningful public participation is required throughout the process. Trust between stakeholders, industry, and regulators is necessary, and increased participation can increase trust. Workshop representatives asked about the nature and level of participation envisioned for the approaches and decision-making process. 
- Environmental and economic goals are equally important. Several participants noted that the approaches should not favor economic goals over environmental goals, and they must provide both environmental and economic benefits.

- Approaches need good performance indicators and measures of success. At least two types of indicators will be required. One type should measure health and environmental improvement and a second should evaluate the performance of the approach(es.)

- Implementation issues must be addressed. The draft approaches contain elements significantly different from those of existing regulatory programs. Most participants observed that some federal laws and regulations would have to be changed in order to implement either of the strawman approaches.

- The three-component structure is appropriate for developing the approaches. Some participants suggested slight variations to the structure, but most agreed that it was an appropriate way to view future-oriented regulatory programs and compare the approaches.

Workshop representatives also provided valuable input on the specific elements of the two approaches. Most of the RBB commenters asked for clarification and details on how the concept of risk would be incorporated. Many participants endorsed the concept of a risk-based approach but questioned how the RBB could be implemented, given the number of technical questions requiring answers. Participants also asked for information on decision-making processes and stressed the need for public participation in all components.

Examples of specific comments and questions related to the RBB, organized by component, include the following:

- Establish the baseline. What criteria would be used to identify residuals of concern? How would the data on residuals and on nearby sources that are needed to characterize the environment be obtained and used in the characterization? What kinds of quality assurance/quality control (QA/QC) procedures would be followed to verify information obtained from various sources?

- Set residual release limits. How would cumulative risk be defined? How would acceptable risk levels be determined? How would uncertainties be addressed? Where would the data come from? (Most participants said they preferred actual data from exposure-point monitors over modeled data.) Under what conditions would reopeners (opportunities to reexamine the limits) occur?

- Assure compliance. How would cross-pollutant and cross-media trading of releases be accomplished and tracked? How would releases eligible for trading be identified? How and where would monitoring be conducted, and how could citizens obtain monitoring results? 
Regarding the NPA, most participants sought additional explanation and clarification, and many stressed the need for public participation in all components. Examples of specific NPArelated comments and questions include the following:

- Establish the baseline. Would pollutants be ranked (e.g., to reflect human health effects) or would they be given equal weight? What would prevent refineries from setting the baseline lower than it actually is in order to show progress relative to that baseline? Could the baseline be considered a target, thereby limiting incentives to improve beyond the baseline levels?

- Set residual release limits. Who would conduct the negotiations, and what would the roles of the negotiators be? How long would the NPA last? How would the NPA account for changes in production and environmental conditions that could increase releases or make goals otherwise inappropriate? How would flexibility be built into an agreement designed to last for several years?

- Assure compliance. What, if any, compliance milestones would the refinery need to meet during the course of the agreement? How would penalties be structured, and could they be assessed before the end of the agreement? How would affected interests be compensated? How would information be made available to the public?

\section{S.3 Revised Approaches}

Most workshop participants stressed the need for more detail and clarification; however, few specifically endorsed or rejected individual components or elements. Thus, the focus was not on modifying a favored approach to reflect recommended changes (an outcome originally expected from the workshops) but on revising both approaches to incorporate the requested detail and clarification. To conduct this task, the findings of other studies and reports on regulatory reinvention and risk assessment were used to answer participant questions and to provide support for specific concepts embodied in the two approaches.

\section{S.3.1 Revised RBB}

The RBB defines allowable releases of pollutants from the refinery such that the total risk resulting from all releases and all sources, when considered in the context of the surrounding community, does not exceed a predetermined total cumulative acceptable risk level. Several sitespecific factors are considered in setting the release limits. These include types of pollutants released from the refinery, individual pollutant toxicities, exposure pathways, and exposed populations. The RBB treats refinery operations as though a bubble surrounded the plant, with source-specific releases coming from the bubble rather than from individual stacks. The approach considers the synergistic and cumulative effects of pollutants released from the refinery and from nearby facilities that affect public health and the environment. Because the controlling factor is total risk, pollutant-specific releases can be modified or exchanged with other releases, as long as the total risks from all pollutants and the individual risks from specific pollutants remain within the acceptable levels. The approach gives plant managers flexibility in controlling releases from disparate sources, as long as total cumulative risk remains acceptable. 
Many of the workshop concerns about the RBB, particularly those regarding the implementation of risk-related provisions, were not trivial. However, given the number of existing and proposed efforts to expand risk-related data collection and the increasing number of government and nongovernment organizations calling for increased emphasis on risk in regulatory reform efforts, the project team believes that these concerns will be resolved and that the RBB will thus be able to be demonstrated, at least on a pilot scale, within the next 15 to 20 years.

Some of the modifications and amplifications incorporated into the revised RBB to address workshop comments include the following:

- Decision making. The revised RBB envisions a decision-making body consisting of representatives from the refinery, the regulatory body, and the local citizenry. The number of representatives on this "RBB Board" should be large enough to represent the views of all stakeholders and small enough to operate efficiently and effectively. The RRB Board would be responsible for agreeing on the baseline, approving the risk-based limits, and approving compliance assurance measures.

- Public participation. The revised RBB incorporates two levels of public participation in all three components. The first level consists of local citizens who would represent broad community values and would take a long-term, active role by participating on the RBB Board. The second level consists of community representatives who might not have the time or desire to be on the RBB Board but would be interested in the process and would contribute by communicating with the community, exchanging information, and otherwise consulting with and for the RBB Board.

- Baseline. The purpose of the revised RBB baseline is to provide information to develop refinery-specific release limits. Although these limits can change, and releases can be exchanged as long as the risks associated with those releases remain within acceptable levels, the baseline provides an initial starting point. To establish the baseline, the RBB Board should direct the following five activities:

1. Develop an inventory of refinery releases.

2. Characterize the environment affected by releases by using information collected under other auspices (e.g., EPA monitoring efforts) combined with information from local citizens, reviewed for scientific objectivity.

3. Characterize pollutants of potential concern by using existing risk assessments and other peer-reviewed scientific literature.

4. Screen and prioritize pollutants and set release limits by using data from other research efforts (e.g., EPA's Risk Screening Indicators Project.)

5. Document and communicate results. 
- Setting limits. The revised RBB sets release limits so that resources are targeted toward activities and releases that provide the greatest contributions to total human and ecological risk. The goal of setting chemical-specific release limits is to ensure that refinery releases, when combined with other pollutants in the environment, will result in a total cumulative risk level that is considered acceptable by all potential stakeholders. Cumulative risk refers to the potential risks presented by multiple stressors in the aggregate; it recognizes that combinations of pollutants from various sources through various environmental media over various time periods affect human and ecological receptors. Several ongoing cumulative risk projects serve as models for setting release limits in the RBB. ${ }^{1}$ While no single existing model can project the total cumulative risk associated with releases of all refinery pollutants combined with all other pollutants in the area to which human and ecological populations may be exposed, the EPA and others are beginning to develop tools to conduct such assessments. Thus, the modeling system to be developed should use existing and to-be-developed information on pollutant toxicities, interactions among pollutants, cause-and-effect relationships, fate and transport, and dose-response relationships in conjunction with site-specific data to calculate total cumulative risk as well as the risks associated with individual pollutants. This cumulative risk evaluation and assessment model (CREAM) will help set release limits that reflect uncertainties in data and methods and will provide a means to track and help assure compliance. The following six steps should guide the RBB Board in setting the limits.

1. Determine acceptable risk, referring to other studies that have defined acceptable risk and incorporating public input.

2. Calculate an initial total cumulative risk estimate based on existing baseline emissions and monitored emissions from surrounding sources and exposure points by using CREAM.

3. For priority pollutants identified in the baseline, establish initial release targets.

4. Develop alternative limits for individual pollutants, allowing for exchanges across pollutants and media, as long as the total risk remains acceptable.

5. Document agreed-upon release limits and make them accessible to the public.

1 Such projects include the EPA's Chicago Cumulative Risk Initiative, which strives to measure and reduce cumulative risk to Chicago-area residents; the EPA's Total Risk Integrated Methodology (TRIM), which is a multipollutant, multimedia, multipathway assessment model to help evaluate and regulate health risks from air emissions; the EPA's 1999 revised Guidance for Conducting Health Risk Assessments of Chemical Mixtures; and the EPA's Human Health Risk Assessment Protocol for Hazardous Waste Combustion Facilities, which assesses risks of hazardous waste combustors from direct and indirect pathways. 
6. Conduct periodic reexaminations of release limits to incorporate new information. Reopeners could be initiated upon the suggestion of interested parties who have documented evidence that a change may be warranted.

- Compliance assurance. The revised RBB gives the refinery flexibility in selecting compliance methods, requiring only that refinery releases do not result in (1) a total cumulative risk that exceeds the agreed-upon acceptable limits or (2) pollutant-specific releases that exceed risk-based maximum levels. The revised RBB assures compliance through the use of direct, real-rime monitoring of releases and resulting concentrations linked to the cumulative risk model, or CREAM. CREAM also tracks pollutant exchanges. Release data and resulting risk levels would be made publicly available via the Internet and would be accompanied by several explanatory reports that highlight refinery actions to reduce pollution, penalties imposed, monitoring data, and public participation activities. The release limits and the penalty structure contain incentives to use exposure-point monitoring rather than modeled data.

- Risk-based release exchanges. A key element of the revised RBB is the ability to exchange or trade releases across pollutants and media on the basis of risk. Such exchanges, which are designed to provide flexibility in meeting environmental goals, are expected to occur primarily within the refinery bubble. However, under certain circumstances, exchanges between the refinery and one or more nonrefinery sources may occur. The ability to exchange is based on the assumption that risk provides the measure (i.e., currency) on which exchanges can be made. CREAM accounts for cross-pollutant and cross-media exchanges by running data on various combinations of the refinery's releases to identify pollutant-specific release limits that would keep total cumulative risk within acceptable levels. Thus, releases that produce high risk would be reflected in an increased cumulative risk, and if that risk exceeded the acceptable risk level, the exchange would not occur. Exchanges could be prohibited for certain pollutants until pollutant mechanisms were sufficiently understood so that exchanges would not result in unacceptable risks. As information on the appropriateness of certain air pollutants for trading is developed, adjustments would be made to account for uncertainties.

- Performance evaluation. The revised RBB includes two types of indicators: environmental indicators and performance indicators. Because the RBB links releases to health and ecological benefits, it can be argued that by design, the approach contains a built-in environmental indicator. However, because the link between releases and risk relies on assumptions, other measures may more accurately indicate changes in human health and the environment. Thus, the revised RBB incorporates additional environmental indicators (e.g., local disease rates linked to refinery emissions, fish-tissue advisories.) Performance indicators (e.g., greater public access to information, cost savings) measure the effectiveness of the RBB in meeting its goals.

- Implementation. The project team and workshop participants identified concerns about RBB implementation that included technology requirements, information for setting riskbased limits, and institutional resistance. Because the revised RBB is not intended to be implemented immediately, increased scientific and technical knowledge, combined with 
expanding regulatory reinvention processes, are expected to help resolve these issues. However, many of the revised RBB provisions (e.g., multimedia approaches, participatory standards development process, use of risk in setting limits) conflict with existing federal and state laws and regulations; therefore, implementing the approach could require Congressional action. Three options for addressing these statutory implementation issues were identified. First, a pilot-study waiver, which could be included in an appropriations bill, would allow the EPA to waive the environmental requirements necessary to implement the revised RBB on a pilot scale. Second, a strategic waiver would allow the EPA to waive compliance for any number of refineries (or other entities) on which the EPA, the regulated entity, and other stakeholders could agree. Third, individual statutory changes would permanently modify specific environmental statutes to enable implementation of reforms such as those contained in the revised $\mathrm{RBB}$.

State laws and regulations may also inhibit RBB implementation, because state rules can be more numerous and stringent than federal rules. Potential implementation constraints and facilitating mechanisms were examined for the State of Texas, where several refineries are located. Although Texas environmental regulations are not considered to be more strict than federal regulations, Texas has some additional requirements that do not exist at the federal level. Nonetheless, Texas is developing regulatory initiatives that could facilitate RBB implementation. These include regulatory flexibility orders, which allow applicants to propose alternatives to current rules; flexible air permits, which allow for plantwide emissions caps; the Texas Risk-Reduction Program, which uses risk assessment techniques to set protective concentration levels in environmental media; and the permitting of "grandfathered facilities," which helps ensure that all sources of releases are identified and that compliance requirements are based on local health considerations.

\section{S.3.2 Revised NPA}

The revised NPA is a goal-based, facilitywide, multimedia regulatory program that incorporates several innovative regulatory concepts found in alternative approaches being implemented in the United States and abroad. The baseline, which includes an inventory of residuals and an inventory of environmental management costs, reflects current, refinery-specific operating conditions and provides a starting point for setting release limits and a benchmark for measuring progress toward meeting those limits. In the revised NPA, release limits are negotiated by a council that includes representatives of the refinery, the regulatory agencies, and local citizens groups. The limits are negotiated on the basis of goals established by state or federal environmental policies or by the parties involved in the negotiations and current refinery operating conditions and releases. The negotiated limits should not exceed existing and anticipated future regulatory limits.

The negotiated limits provide the basis for the facilitywide permit, or negotiated performance agreement, which would remain in effect for a negotiated period of time of at least 10 to 15 years. At the end of the period, the refinery would be expected to have met its release 
reduction goals. To provide for continuous improvement, the limits could become more stringent over time, and milestones could be set to assess progress.

The revised NPA gives the refinery more flexibility in meeting its environmental protection goals than does the current system. Thus, rather than requiring the refinery to meet a variety of source-specific technology, permitting, scheduling, and other requirements, the revised NPA allows the refinery to use the most cost-efficient and effective means it can identify to meet the NPA goals. The revised NPA envisions an electronic reporting system that is integrated with emissions monitors to reduce staff burden and increase reporting accuracy. Compliance is assured, in part, through publicly available reports.

Many of the elements of the revised NPA are similar to regulatory innovations that have been developed and successfully implemented in the United States and abroad. These include the EPA's Project XL Program, which offers a facility increased flexibility in how it meets its environmental responsibilities (usually via relief from a specific regulation) in exchange for "superior environmental performance"; the Netherlands Covenants Program, in which representatives of industrial sectors negotiate with regulatory agencies to meet certain environmental goals over a specified time period; and comprehensive state environmental permits. Several states (e.g., New Jersey, Oregon, Texas) have begun implementing comprehensive facilitywide or multimedia permits to reduce regulatory burden, increase operational flexibility, and provide equal or better environmental performance. Each of these programs shares common elements with the revised NPA. However, none is identical in all aspects. For example, XL projects are generally much narrower in scope than the revised NPA, which seeks to substitute negotiated limits for all pollutants from all media in a single agreement. In the Dutch Program, covenants are signed at the sector level rather than the facility level, and those covenants are tied to national-level environmental goals. The NPA envisions agreements negotiated at the refinery level, and the revised NPA calls for the negotiated limits to reflect goals set at the beginning of the process. However, because the United States has not developed national-level environmental goals to the extent that the Netherlands has, the revised NPA cannot be linked to these goals as closely as may be desired. Comprehensive state permits share with the revised NPA the concept of covering pollutants from all media in a single, facilitywide permit, but they generally rely less on public participation and negotiation than does the revised NPA. Regardless of the differences between these programs and the revised NPA, each provides support for the development and testing of the approach.

Most of the workshop comments on the NPA pertained to the need for additional detail, explanation, and clarification of the NPA elements. They also stressed the need for meaningful public participation in all three components. Some participants suggested improving the NPA structure by adding an initial component to establish refinery-specific NPA goals. Some of the additional details and clarifications incorporated into the revised NPA include the following:

- Public participation. The revised NPA includes public participation in all three components via a refinery-specific "NPA Council." The NPA Council would consist of representatives from the refinery, the regulatory agencies, and the local citizenry. A suggested model would include six voting members and roughly 14 to 19 nonvoting members, for a total of 20 to 25 members. The voting member component would be 
similar to the RBB Board in that it would include representatives (suggested to be two each) from the refinery, the regulatory agencies, and the local citizenry. Nonvoting members could include other refinery or parent company representatives, additional regulatory agency representatives, additional citizens group representatives, local elected officials, and representatives of national environmental groups. The NPA Council would be responsible for the success of the entire NPA process at a given refinery. Thus, it would be responsible for formulating the initial goals, setting the baseline, negotiating release limits, and assuring compliance.

- Establishment of goals. Some workshop participants suggested that a goal-setting component added to the beginning of the process would help guide the baseline-setting process, assist in negotiating the release limits, and aid in scaling incentives and penalties for compliance assurance. Goals could be expressed in such terms as types of pollutants to be reduced, environmental impacts to be mitigated, pollution prevention expectations, or anticipated new technology implementation. The revised NPA includes the establishment of refinery-specific NPA goals as a first step in the NPA development process.

- Baseline. The purpose of the revised NPA baseline is to provide a benchmark for setting release limits and for assessing progress in meeting those limits. As such, it should reflect current operating conditions at the refinery. To establish the baseline, the NPA Council would oversee the following three activities:

- Develop inventory of refinery residuals.

- Develop inventory of refinery environmental management costs.

- Document results.

Facilitywide observations, interviews, record reviews, and other methods would be used to identify current residuals, regardless of whether the residuals were currently regulated. Designating the release sources would facilitate the identification of pollution prevention opportunities. To ensure that the scope of this potentially resource-intensive task would be manageable and to account for potential differences in pollutant toxicities that might affect how the limits are set, the NPA Council might use a screening process to prioritize residuals to be measured in the baseline. Factors to consider in such a screening process could include national goals, human health effects data, and pollutant interactions with other constituents in the local environment. The results of the residuals baseline should be publicly available (e.g., by posting on the Internet).

Because any benefits associated with implementing an alternative regulatory program that would be realized by a refinery would be measured by reduced costs, an accurate identification of baseline costs could give the refinery a realistic basis on which to measure the impacts of the revised NPA. Environmental costs are frequently misallocated (generally to overhead) and thus can be difficult to track, 
evaluate, and reduce. The NPA Council should consider using total cost allocation methods described in the current accounting literature to facilitate the cost collection activities. The NPA Council would need to consider confidentiality issues related to giving the public access to cost data and should review confidentiality programs in states such as New Jersey where such programs have been successfully implemented.

- Setting limits. The revised NPA uses various regulatory innovations to form the basis of a negotiated agreement and incorporates a time period long enough to allow the refinery to implement effective and efficient design or process changes to meet mutually agreedupon goals. The revised NPA calls for limits to be set on a refinery-specific rather than a source-specific basis. In contrast to the revised RBB, however, the revised NPA does not allow releases to be traded across pollutants and media within the bubble. Nonetheless, the revised NPA is a multimedia permit, because it incorporates release limits for multiple pollutants and media in a single document. In setting the limits, the NPA Council should consider such factors as the refinery-specific NPA goals, baseline emissions, current regulatory requirements, anticipated regulatory requirements, recent investments the refinery may have made to reduce emissions, and potential changes in refinery production. In addition to pollutant-specific limits, the permit might also include performance measures such as the introduction of new process or control technologies, incorporation of stakeholder views in the decision-making process, reduced time and effort for compliance assurance activities, and cost savings to the refinery and the regulator. The negotiated performance measures should be publicly available through venues such as the Internet.

- Compliance assurance. The revised NPA requires more interaction among stakeholders to assure compliance than do traditional permitting approaches. The NPA Council would develop compliance assurance mechanisms, which might include public participation in compliance assurance activities such as assisting in monitoring inspections or developing meaningful reports. The revised NPA does not necessarily require less reporting but does require more efficient, effective, and transparent reporting. For example, the linking of NPA reports with electronic monitoring results should enhance speed and accuracy. Revised NPA reports should also include information on pollution prevention results, other refinery actions taken to meet the limits, and examples of enhanced public participation.

The revised NPA provides for incentives. For example, the amount or frequency of reporting could be reduced if the refinery were to meet the goals ahead of schedule or produce fewer releases than allowed in the agreement. The revised NPA also specifies actions for which penalties may be assessed and the nature of such penalties. The penalties could be structured to increase or decrease depending on the nature and extent of the violation. For example, penalties could be assessed that link the cost per ton of pollutant exceeding the limit to the potential harm of the pollutant. The revised NPA provides that monetary and nonmonetary penalties can be used to benefit local communities. For example, the NPA Council could direct penalties to specific projects (e.g., wetlands restoration, implementation of disease 
detection and treatment programs) within the local community. The agreement might also provide for severe violations to result in a reversion to the existing command-and-control regulations in addition to high monetary penalties.

- Resource requirements. Many workshop participants noted that developing and implementing the revised NPA would be time- and cost-intensive. Reports on resources used to implement typical XL projects support these claims. However, most of the time and dollars for the NPA will be spent in the early stages (convening the NPA Council, setting the baseline and limits). Over the longer term, resource requirements are expected to decrease as stakeholders move along the learning curve, see the results of similar reinvention projects, and realize the benefits of electronic monitoring and reporting. The NPA Council could also limit resource requirements by using the established goals to prioritize the pollutants for which limits would be set.

- Jurisdictional and legal issues. The current environmental protection system in the United States involves numerous responsibilities shared by different levels and jurisdictions of government. Implementing the revised NPA would require, at the least, a greater degree of communication and cooperation among these various regulatory agencies than typically exists today, and it could entail a complete reworking of the regulatory structure to accommodate a facilitywide, multimedia approach. Optimism about changes in these directions derives from experience in other countries, such as the Netherlands, where the Covenants Program has required multiple federal, state, and local agencies covering various subject areas to meet together with individual companies to develop environmental plans consistent with Covenant goals. The revised NPA, as does the revised RBB, envisions concepts and actions (e.g., multimedia permits, incentives for pollution prevention) that conflict with certain existing environmental statutes. Thus, the revised NPA requires that some of these laws be modified before it can be implemented. Such modifications could be accomplished by the same methods as those suggested for the revised RBB (i.e., pilot study waivers, strategic waivers for a number of refineries, or amendments to resolve conflicting aspects of individual statutes).

\section{S.4 Conclusions}

Two future-oriented environmental regulatory program approaches for petroleum refineries were developed by the ETI project team. These approaches - a risk-based approach (the RBB) and a goal-based approach (the NPA) - strive to meet the potentially conflicting goals of environmental responsibility and economic performance by incorporating pollution prevention and technology innovation. A variety of government and nongovernment studies support the concepts of regulatory reform reflected in the ETI approaches. The alternative environmental regulatory programs described in this report represent compilations of EPA and other reinvention activities that can provide tools to integrate a diversity of reform ideas.

To address workshop comments, the results of other regulatory reform and related research activities were considered. The approaches were revised to a point where they could benefit from further comment and eventual pilot testing. Conducting a pilot test at an actual refinery or a 
hypothetical case study would advance the iterative and integrated process of testing ideas, generating feedback, and continually refining and improving the approaches.

Several individuals and organizations (e.g., state regulators, national environmental groups, the EPA's Regulatory Reinvention Office) have expressed interest in participating in or tracking further development and implementation of the approaches. Perhaps more importantly, several local citizens groups have endorsed the ETI approach, thereby increasing the potential of acceptance and implementation.

The development of the two future-oriented alternative environmental regulatory approaches for petroleum refineries also produced benefits beyond those originally intended. These include exchanging information with public interest groups on scientifically based approaches to environmental regulation and providing lessons learned to the EPA for its broader reinvention efforts. There is also a potential for applying these prototypes to other industrial sectors. 


\section{Section 1 Introduction}

The U.S. Environmental Protection Agency (EPA), Congress, and other organizations are exploring initiatives to address the perceived inflexibility of existing environmental laws and regulations in various industries. At the same time, the petroleum refining industry is facing challenges that include an increased demand for more highly refined products, a decline in feedstock quality, and uncertainties in petroleum industry economics and environmental requirements. An environmental regulatory program for the petroleum industry should reflect efforts to reform existing regulations and consider new approaches for meeting environmental and economic performance objectives in the context of these operational challenges.

The EPA and the Office of Policy and the Office of Fossil Energy in the U.S. Department of Energy (DOE) have sponsored a project to develop alternative, future-oriented environmental regulatory programs for refineries. The project began as a U.S. Environmental Technology Initiative (ETI) grant. The ETI seeks to accelerate environmental protection, strengthen the U.S. industrial base, and increase exports of U.S. technologies and expertise.

The ETI petroleum refining project used the expertise of DOE and its national laboratories to develop two alternative, multimedia, environmental regulatory approaches for existing refineries that will operate in the future under the conditions noted above. The ETI project differs from other EPA regulatory reform efforts, which are directed toward short-term solutions. It addresses future operating conditions and regulatory programs that encourage technology innovation and use. The ETI project focuses on environmental requirements at refineries; it does not address occupational health and safety concerns, reformulated fuels requirements, or other product-related regulations.

The ETI project team responsible for developing the alternative multimedia regulatory approaches includes staff from the Environmental Assessment Division of Argonne National Laboratory and from Analytical Services, Inc., in Columbia, Maryland. A manager in the DOE Office of Policy directed the project with support from a manager in the DOE Office of Fossil Energy.

This report presents the results of the project. It contains six sections in addition to this introductory section. Section 2 provides background information on the current environmental regulatory environment and the projected future operating environment for refineries. Section 3 describes two draft strawman approaches: a goal-based approach and a risk-based approach. Section 4 describes (1) the way that workshops with representatives from regulatory, industry, local community, and environmental groups that could be affected by the alternative approaches were used to obtain input on the approaches and (2) the findings of those workshops. Sections 5 and 6 describe how the approaches were revised to reflect the comments from the workshops for the RBB and the NPA, respectively. Section 7 provides the conclusions of the study. 


\section{Section 2 Background}

In the early 1970s, Congress enacted several environmental laws in response to the public's demands to reduce air, water, and solid and hazardous waste pollution in the United States (e.g., the Clean Air Act [CAA] and Clean Water Act [CWA]). To implement the laws, the EPA developed regulations that are generally characterized as single-medium, technology-based, "end-of-pipe" requirements. As such, they are typically enforced without regard to cross-media and multimedia impacts and site-specific conditions, and they often limit incentives for new technology development and use. Although most government and private-sector observers agree that these environmental regulatory programs significantly improved many aspects of the nation's air, water, and land resources, they also express concern that their ability to meet the challenges posed by new technological knowledge and changing environmental concerns is being stretched. For example, the single-medium orientation of the current regulatory system limits the ability to prevent negative cross-media and multimedia environmental impacts. These impacts occur when cleaning one environmental medium (e.g., air contaminated by industrial emissions) leads to unintended adverse environmental impacts in other media (e.g., pollutants from waste generated during air pollution control leach into the groundwater). The current approaches may also not address many risks (e.g., health impacts on the elderly). In addition, they lack incentives to develop and use new technologies. Moreover, the current regulatory approaches must meet the challenges posed by increasingly well-informed citizens and newly emerging environmental issues (such as persistent bioaccumulative toxics, aesthetics, and sensitive populations). Meeting these challenges in more competitive economic environments requires the development and evaluation of fundamentally new, scientifically based regulatory approaches that recognize economic constraints, new knowledge of environmental effects, and the benefits of using new technologies.

During the past few years, government organizations, think tanks, industry trade associations, and others have recognized these problems, and many have undertaken efforts to suggest new approaches. Appendix A highlights several of these efforts. However, most of these regulatory reinvention efforts are reactive, and many strive for 12-month to 18 - month fixes of specific regulations.

The ETI project has taken a different direction. Rather than attempting to address specific problems with existing regulations, this effort emphasizes the need to be proactive and future oriented. Its time frame -20 years or more - is much longer than those of most other regulatory reinvention activities. It reflects the projected future operating environment for petroleum refineries, recognizing the challenges presented to the industry by changing feedstocks, product demands, technological needs, and economic conditions. It also seeks to address changing environmental requirements, especially multimedia impacts and the benefits of preventing pollution before it occurs rather than removing it after it has been generated. To develop the alternative regulatory approaches, the project team used an interactive and iterative process, which is summarized below and presented graphically in Figure 1. 


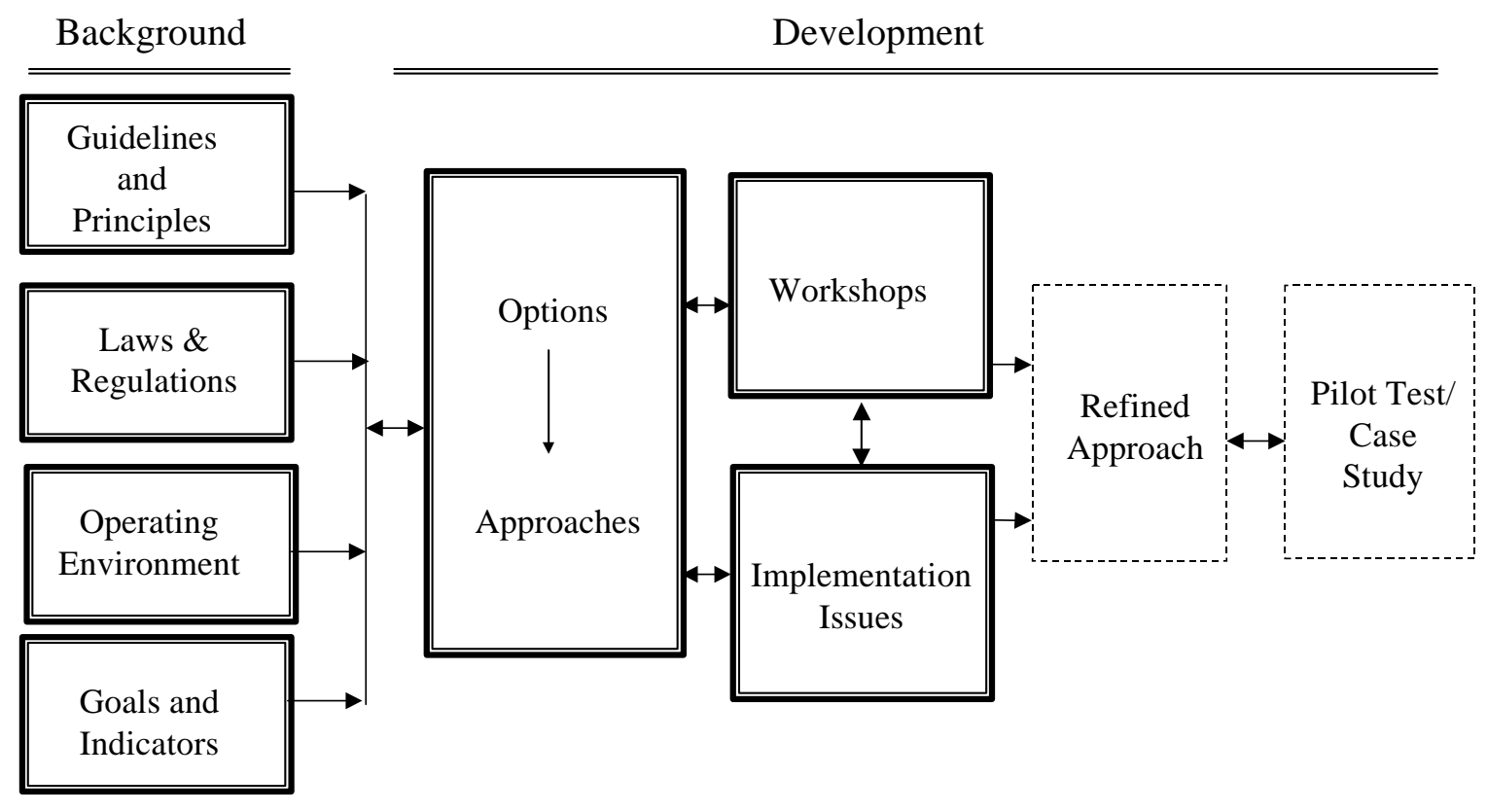

Figure 1 Regulatory Alternative Development Process

First, the project team established a set of guidelines and principles to bound the study and set parameters for developing the approaches. For example, one of the guidelines provided that the approaches would pertain only to refinery activities, and therefore, regulatory impacts and changes associated with exploration and production or of products would not be addressed in this study. Appendix B lists the guidelines and principles. Background investigations on regulatory needs and the projected future operating environment for refineries were then conducted to provide a basis for identifying and evaluating alternative approaches. A set of goals and indicators to help compare and assess the alternatives was also developed. After collecting and assessing this background information, the team identified and evaluated numerous options to help meet the needs of an alternative regulatory approach. From these options, two separate thematic approaches - a risk-based approach and a goal-based approach - were distilled. Before expanding or developing details for the approaches, the project team sought input and feedback from potentially affected parties. To do so, a series of workshops with seven separate groups of stakeholders was conducted. The team also presented the approaches to a meeting of the Petroleum Refining Subcommittee for the Common Sense Initiative. On the basis of the findings from these meetings and supplemental research on potential implementation issues, the project team then revised the two draft approaches to address concerns raised by potentially affected stakeholders and to provide details for further evaluation. Thus, the development process was interactive in that it sought and used findings from meetings with potentially affected parties, and it was iterative in that findings from various steps (e.g., workshop findings) helped modify the approaches. The team hopes that the revised approaches will benefit from continued review by stakeholders and that pilot testing with a real refinery or a hypothetical case study will provide further opportunity for continued improvement and refinement. The following sections summarize the findings of the background investigations. 


\subsection{Regulatory Environment}

To develop regulatory approaches that would optimize the chances for cost-effective results, the project team needed to understand the environmental laws and regulations governing the control and reporting of air, water, and waste releases from petroleum refineries. Environmental laws and regulations directed at product specifications or occupational health and safety were not addressed. After reviewing the laws and regulations within its purview, the team summarized major statutes and regulations currently affecting refineries, recent rule makings and impending regulations expected to affect petroleum refineries, barriers and incentives to pollution prevention, and opportunities for innovation. It found that the most technologically, economically, and administratively burdensome environmental laws affecting and projected to continue affecting petroleum refineries are the CAA and the Clean Air Act Amendments of 1990 (CAAA), the CWA, and the Resource Conservation and Recovery Act (RCRA.) The regulations associated with these laws either apply specifically to the petroleum refining sector, such as effluent guidelines and other water quality standards for the petroleum refining point-source category, or they apply to all industrial sectors, such as standards for hazardous waste generators. Petroleum refiners must comply with permitting processes for air emissions and water discharges under state operating-permit programs. Many refiners also require RCRA permits (e.g., for operating hazardous waste incineration units.) Appendix $\mathrm{C}$ summarizes environmental laws and regulations affecting the petroleum refining industry.

Implementing these laws and regulations has improved environmental conditions significantly. However, most observers of the refining industry agree that changes to these laws and their implementing regulations (which have evolved in response to Congressional laws and mandates written over the past 25 years) could reduce overlap, increase cost-effectiveness and efficiency, and maintain environmental protection. For example, most regulations pertain to a single environmental medium and require residual releases to be less than or equal to the releases that would result from the application of a specific control technology. As a result, the current regulatory system provides refiners with few incentives to prevent pollution or to try alternative means and technologies to reduce environmental degradation. The maximum achievable control technology (MACT) requirements mandated by the CAAA are commonly cited as regulations focused on single pollutants that result in duplication and limit incentives to develop pollution prevention or new technological approaches to reduce toxic air pollution. ${ }^{2}$ Requirements to reduce pollution in one medium may actually increase pollution in one or more other media, so that actual pollution (measured in terms of health or ecological impact) is not necessarily reduced. On the basis of these findings, the project team concluded that an alternative regulatory approach should provide refiners with the flexibility to meet environmental protection goals through a multimedia program that reduces redundancies and overlap and provides incentives for pollution prevention.

2 For example, the Northeast States for Coordinated Air Use Management (NESCAUM) and the Northeast Waste Management Officials' Association recently reported to the EPA that MACT rules do not adequately support pollution prevention, are difficult to integrate with other regulatory programs, create disincentives for pollution prevention, and have resulted in only limited reductions of hazardous air emissions in the Northeast (NESCAUM 1999). 


\subsection{Future Operating Environment}

To develop approaches consistent with the needs of next-generation refineries, the project team needed to understand and make assumptions about the future refinery operating environment. Although refiners may expand or modify existing operations, no one expects new refineries to be built in the United States in the foreseeable future. Thus, the term "nextgeneration refineries" refers not to fully reengineered future refineries but to the refineries of today operating within the next 20-plus years. The team identified four key factors expected to affect refineries operating in the future. It also identified the implications of these factors that should be addressed by a regulatory approach. The factors — feedstock, product, technology, and economics - are summarized below.

1. Feedstock. The feedstock reaching U.S. refineries is changing as a result of decreasing domestic crude resources. In general, the crude is becoming heavier (Figure 2), and the sulfur content is increasing (Figure 3). These trends are expected to continue for the foreseeable future.

2. Product. Responding to reformulated fuels requirements mandated in the CAAA and reduced demand for heavier fuels, refinery products are becoming lighter. Figure 4 shows that recent and projected growth in worldwide refinery product demand is for the lighter products.

3. Technology. Refiners will need new technologies to produce increasing amounts of lighter products from poorer-quality feedstocks. Continued technological development is expected in the following three key areas:

- Technological changes to make specific refinery processes more efficient (e.g., new catalysts to increase product yield and quality);

- Technological changes responding to environmental concerns at the refinery (e.g., improved sulfur removal processes); and

- Technology improvements for overall refinery operations (e.g., with electricity deregulation, more refiners may sell electric power in addition to products).

4. Economics. Determinants of refinery economics include the following:

- Profitability. U.S. refiners generally exhibit lower profits relative to other parts of the petroleum industry and to foreign refiners.

- Capital expenditures. Installing new capital equipment can increase the flexibility of a refinery to process various crudes, but capital costs cannot always be recovered by increasing the price of products.

- $\quad$ Price of crude. Higher crude prices reduce refinery profit margins. 


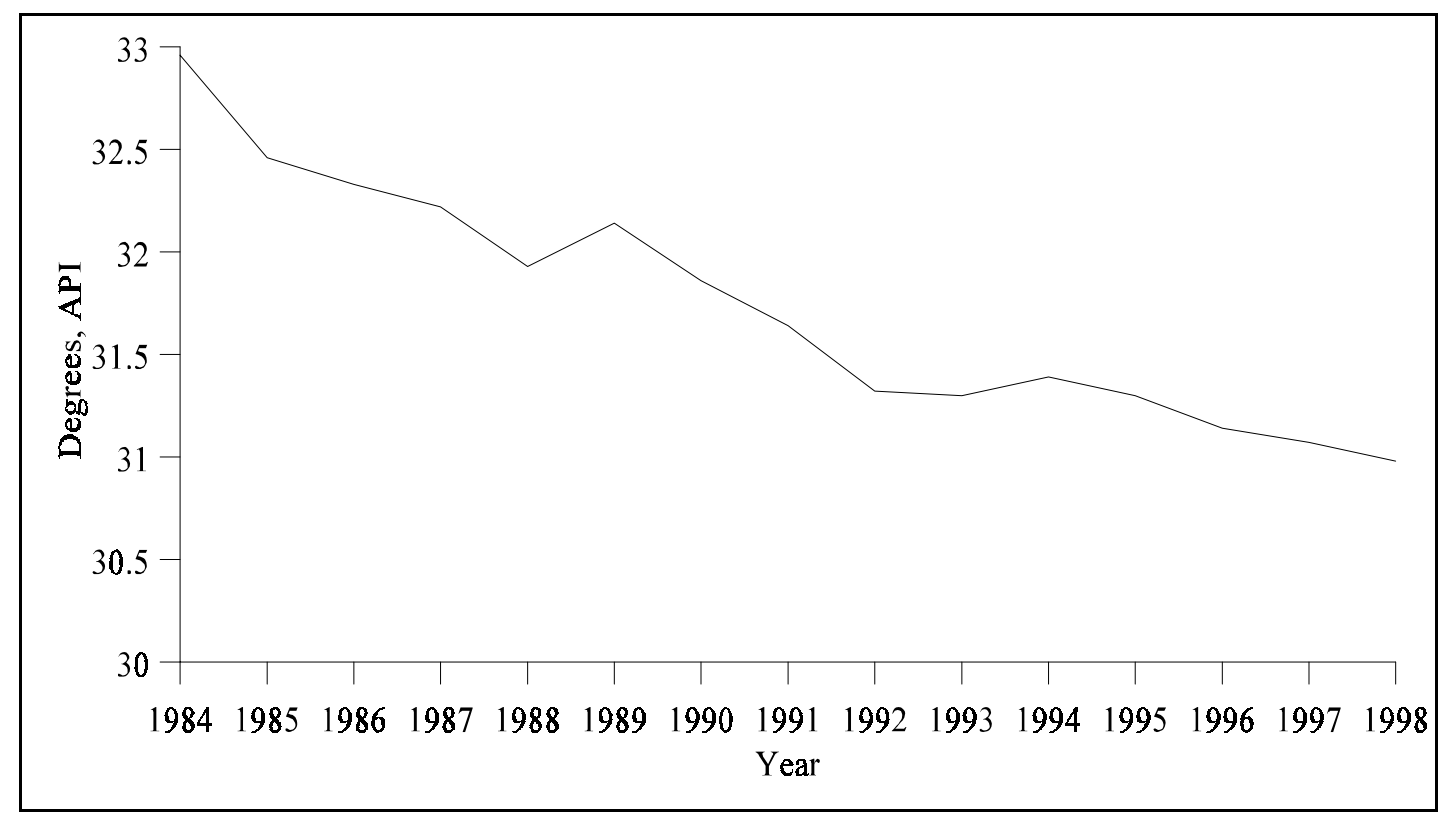

Figure 2 Trends in American Petroleum Institute (API) Gravity, 1984-1998

(Source: Energy Information Administration, 1984-1998) Note: API gravity is an arbitrary scale that measures the density of liquid petroleum products. It is measured in degrees API; the formula is degrees API $=(141.5 /$ specific gravity $)-131.5$. Specific gravity is the density of a material divided by the density of water. The lower the API gravity, the heavier the material.

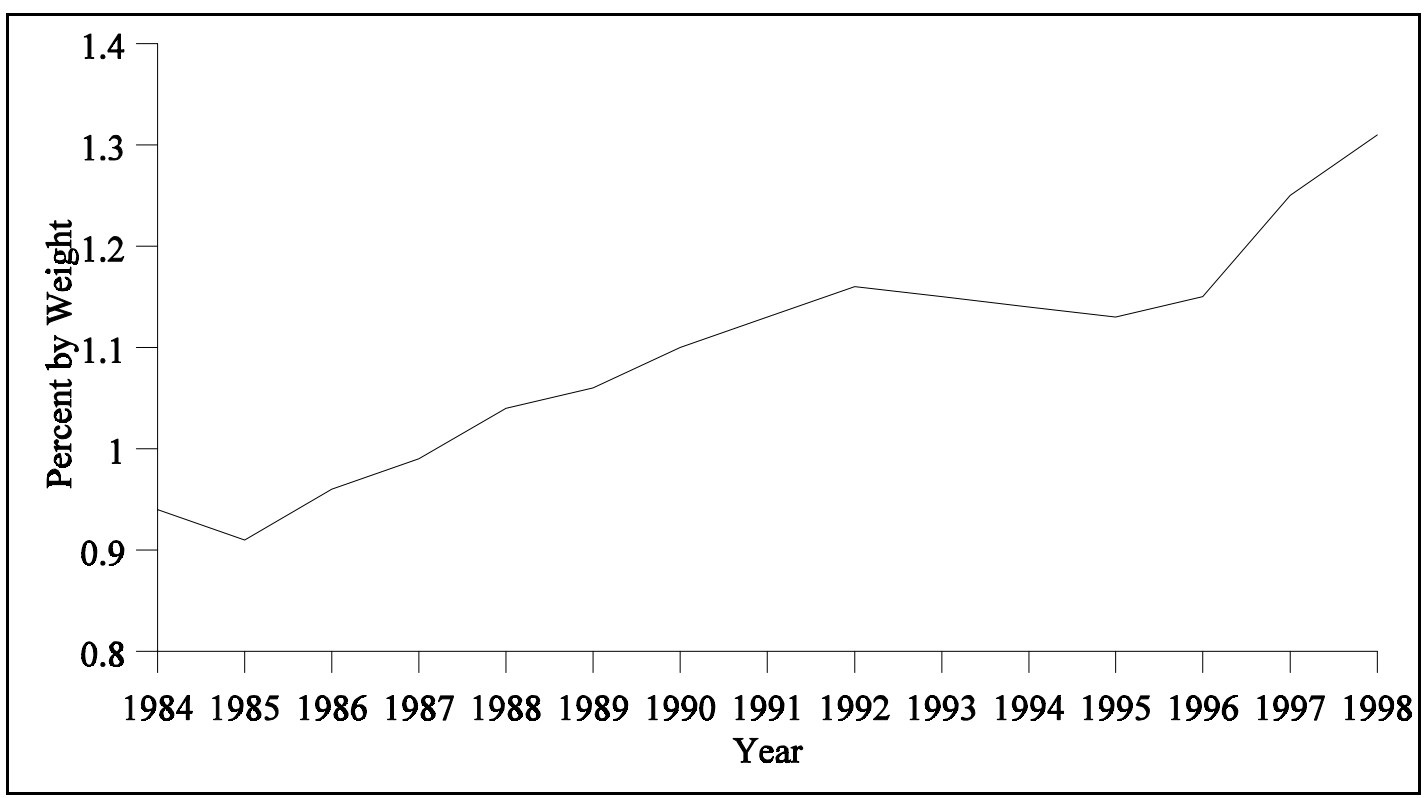

Figure 3 Trends in Sulfur Content, 1984-1998 (Source: Energy Information Administration, 1984-1998) 


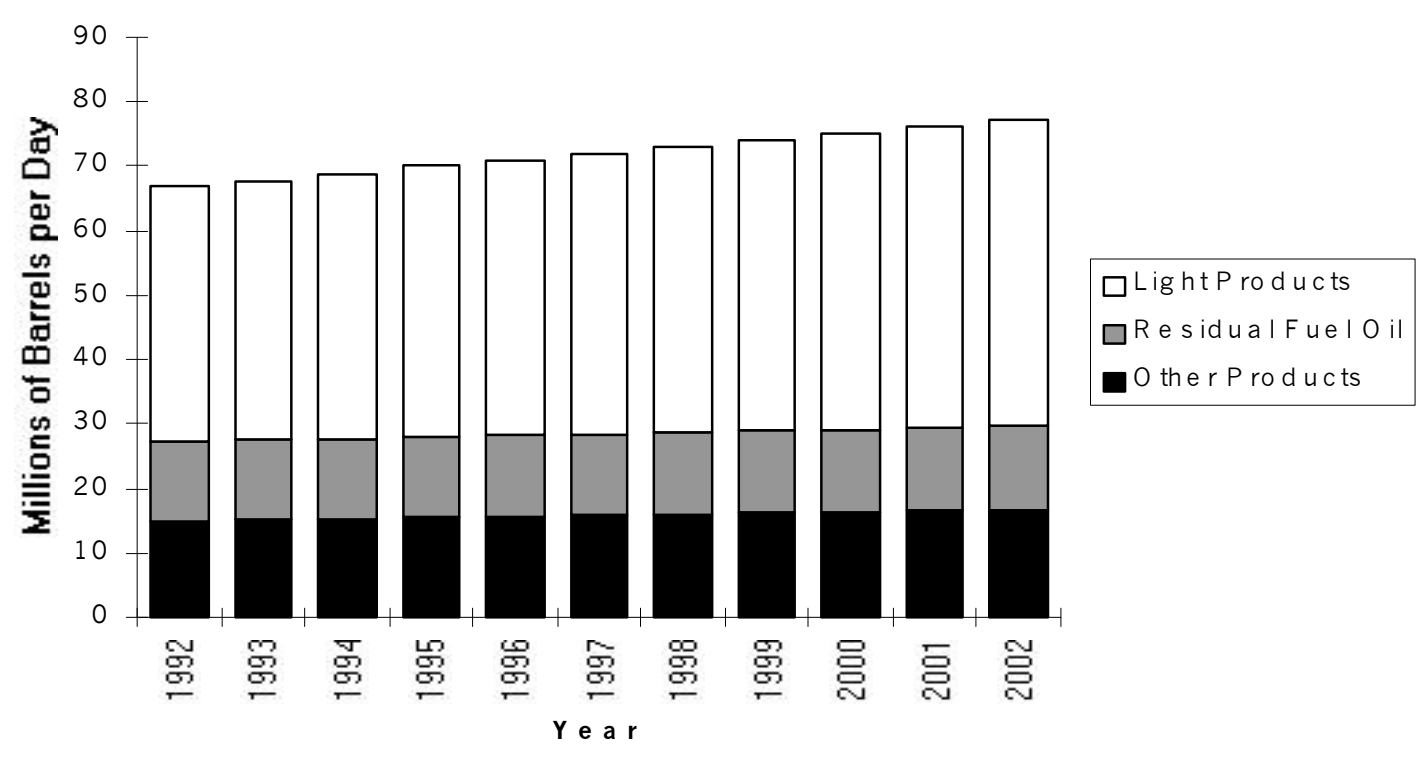

Figure 4 Worldwide Trends in Refinery Product Demand (Source: Data from Dosher and Carney 1994)

- Environmental regulations. Environmental regulatory costs affect refinery economics. For example, unexpected changes to environmental regulations can negatively affect cash flows.

These and other factors will continue to affect refinery economics. However, significant uncertainties associated with these factors limit the ability to predict the future operating environment of refiners with respect to economics.

In summary, the need to balance poorer-quality crudes with increasing demands for lighter products, the urgency to use new technologies, and economic uncertainties characterize the operating environment for future refineries. To be compatible with such a future, the project team concluded that alternative regulatory approaches should provide flexibility, recognize and incorporate the benefits of developing and using new technology, consider refinery-specific rather than source-specific release limits, and reduce costs.

\subsection{Goals and Indicators}

To assess and compare alternative regulatory approaches, the project team established a set of goals and indicators. Goals reflect the purposes of the approach, and indicators measure whether a regulatory program meets those goals. In no particular order, the following goals reflect the guidelines and principles established at the beginning of the development process: 
- Environmental responsibility. The approaches should provide for the protection of public health and the environment; they do not need to explicitly address worker health or safety.

- Economic performance. No regulatory approach should weaken the economic performance of U.S. refineries. A fundamental intent is to decrease, or at least not increase without commensurate benefits, environmental regulatory costs.

- Pollution prevention technology. Pollution prevention technology should be employed to help meet the goals of environmental responsibility and economic performance.

On the basis of criteria that reflect the objectives, scope, and resources of the project, indicators to show the extent to which alternative approaches would achieve these goals were selected. For example, data availability was a criterion. Also, indicators relying on numerical projections were avoided, because the project resources could not support the requisite model development. In addition, the indicators had to show a direct link between the contribution of the regulatory program and the achievement of the goal. Thus, an increase in profits was not an acceptable indicator because profits can reflect numerous nonregulatory factors, such as the price of crude. Appendix D describes the suggested goals and indicators. 


\section{Section 3 Strawman Approaches}

After summarizing the methodology used to draft alternative regulatory approaches, this section highlights the elements of the two resulting strawman approaches: the Risk-Based Bubble (RBB) and the Negotiated Performance Agreement (NPA.) Because the overall methodology for developing the approaches was participatory and iterative, the project team created draft, framework-level strawman approaches to serve as starting points that could be revised and more fully developed on the basis of input from various stakeholder groups. Thus, the strawman approaches presented in this section and to the workshop participants (see Section 4) are intentionally skeletal; they are further developed in Sections 5 and 6.

\subsection{Methodology for Developing Strawman Approaches}

By following the guidelines and principles and using the information on the regulatory and future refinery operating environments, the project team identified more than 60 options, or elements, that could make up a regulatory program for next-generation refineries. These options were diverse. Examples included reducing toxic releases by certain amounts, risk-reduction approaches, exchanging delayed compliance for greater future performance, and using International Organization for Standardization (ISO) 14000 environmental management systems. Options also included implementation alternatives and procedural improvements. To narrow and focus these options, each one was evaluated against the indicators described in Section 2.3. Because the team strove to remain cognizant of the goals, most of these options satisfied the indicators and hence met those goals. Thus, while the evaluation eliminated few of the options, two thematic paradigms, a risk-based paradigm and a goal-based paradigm, resulted from the distillation.

Each thematic paradigm was then developed into a draft, or strawman, approach that consisted of three common components, which are listed below and shown graphically in Figure 5:

1. Establish the baseline,

2. Set residual release limits, and

3. Assure compliance with the regulatory program.

The risk-based approach and the goal-based approach share this common structure. However, the details (the elements or options) that make up each individual component often differ for the two approaches. The previously identified 60 options provided the source of most of the specific 


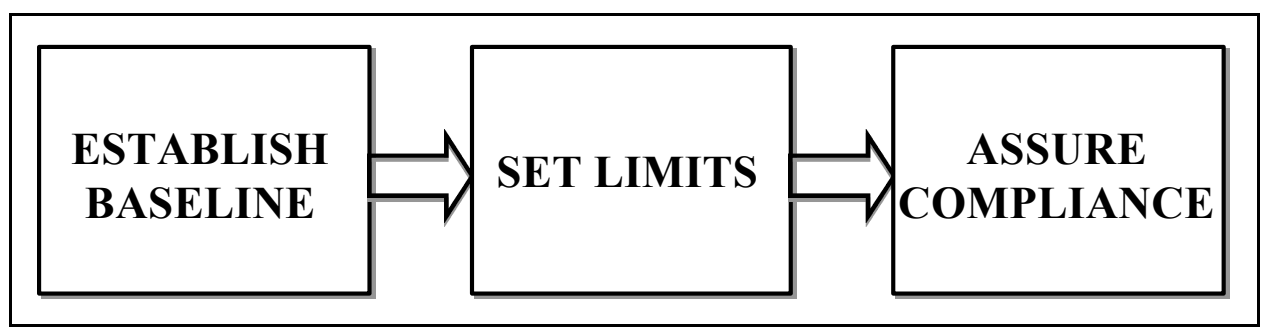

Figure 5 Three-Component Regulatory Approach Structure

elements contained in the two approaches. ${ }^{3}$ The remainder of this section describes the two strawman approaches.

\subsection{Two Strawman Approaches}

Each strawman approach differs thematically from the current system. In general, the current system reflects existing laws and regulations, uses technology-based limits, and provides few incentives for alternative methods to protect public health and the environment (Table 1).

The two alternative approaches are the RBB and NPA. In the RBB, risk provides the basis for setting release limits. As a result, releases can be traded across environmental media and pollutants. The NPA is a goal-based, facilitywide, multimedia regulatory program. Release limits in the NPA are negotiated on the basis of current and expected future regulations, and the resulting agreement serves as a facilitywide multimedia permit. The following subsections summarize elements common to both the RBB and NPA, elements unique to the RBB, and elements unique to the NPA. The strawman approaches were intentionally developed to be fairly broad; they were designed to provide basic concepts and structures to which potentially affected parties could react. Input and comments obtained during workshops were integrated into the two basic approaches later.

\subsubsection{Elements Common to Both the RBB and the NPA Strawman Approaches}

The RBB and NPA strawman approaches share some common elements. For example, in establishing the baseline, both strawman approaches identify residuals for which release limits

3 Because the approaches are future-oriented and not tied to existing laws or regulations, the terms used here in conjunction with the approaches may not be consistent with those used in existing laws and regulations. For example, here the terms "pollutants," "contaminants," and "residuals" are used interchangeably. These terms refer to any element, substance, compound, or mixture in any medium or phase (e.g., solid, liquid, gas), which has been released from the refinery (or other facility) and which, upon exposure to any organism, can be expected to cause adverse human health or environmental effects. When one of these substances enters the environment as the result of escaping, leaking, leaching, or being emitted, spilled, emptied, dumped, disposed of, or discharged (in a form other than a product), it becomes a release. 
Table 1 Key Elements of Current Regulatory Program

\begin{tabular}{|lll|}
\hline \multicolumn{1}{|c|}{ Establish Baseline } & Set Limits & Assure Compliance \\
\hline $\begin{array}{c}\text { C Identifies residuals for which } \\
\text { release limits must be set }\end{array}$ & C Often bases limits on technology & C Uses single-medium permits \\
$\begin{array}{c}\text { C Identifies residuals on the basis } \\
\text { of statutes or regulations }\end{array}$ & C Uses one limit for many cases & C Assesses penalties for \\
$\begin{array}{c}\text { n Identifies residuals separately } \\
\text { for each medium and source } \\
\text { within a facility }\end{array}$ & $\begin{array}{c}\text { C Typically sets limits for individual } \\
\text { sources on the basis of current }\end{array}$ & $\begin{array}{c}\text { C Is based on only limited use of } \\
\text { incentives }\end{array}$ \\
& C Provides only limited \\
& & opportunities for innovation \\
\end{tabular}

must be established, and these residuals are not limited to those currently reported or regulated. In addition, the refiner and the regulator jointly establish the baseline.

The limits are specified on a facilitywide basis rather than a source-specific basis, and they are specified in a process conducted jointly by the refiner and the regulator. In both cases, a facilitywide permit documents the release limits and the parameters of those limits.

For both approaches, incentives provide the basis for ensuring compliance, and flexibility in the compliance method is encouraged. Penalties apply if releases exceed the limits, and reporting requirements are streamlined relative to current practices.

\subsubsection{Elements Unique to the RBB Strawman Approach}

The RBB contains several elements that distinguish it from the current system and from the NPA. For example, establishing the baseline in the RBB includes not only identifying environmental residuals but also characterizing the general environmental conditions surrounding the refinery. Information on site-specific environmental conditions and receptors is the input used to set the risk-based limits. In characterizing site-specific environmental conditions, the refiner and regulator solicit data from potentially affected parties.

In the RBB, the refinery and the regulator set release limits on the basis of risk to public health and the environment; they do not use current regulatory requirements to set those limits. The process for setting limits starts with establishing the residuals of concern on the basis of toxicity or other known health or environmental effects. The process then establishes acceptable cumulative health and ecological risk on the basis of the site-specific receptors and conditions identified in the baseline. Best available scientific information is used to estimate risk. The process then employs risk modeling to establish pollutant-specific release limits, which are designed to keep risk within acceptable levels. The regulator and the refinery reexamine the limits periodically to incorporate information on new or changed conditions, such as the toxicities of residuals or other emissions entering or leaving the area. 
Compliance in the RBB is assured by allowing each refinery to select or develop its own approaches for meeting the limits. The use of electronic monitors linked to risk models allows the regulator to verify that the limits are not exceeded. A key difference between the strawman approaches in assuring compliance is that in the RBB, refiners are allowed to trade releases across media and pollutants on the basis of risk. Linking of electronic monitoring results to risk models to document allowable trading facilitates such trading. Table 2 summarizes key elements of the RBB strawman approach.

\subsubsection{Elements Unique to the NPA Strawman Approach}

The NPA strawman approach contains several unique elements. For example, in addition to inventory of current releases, the NPA baseline also requires an inventory of environmental management costs. The baseline thus provides a starting point for identifying more cost-effective environmental management options.

Table 2 Key Elements of the Risk-Based Bubble Strawman Approach

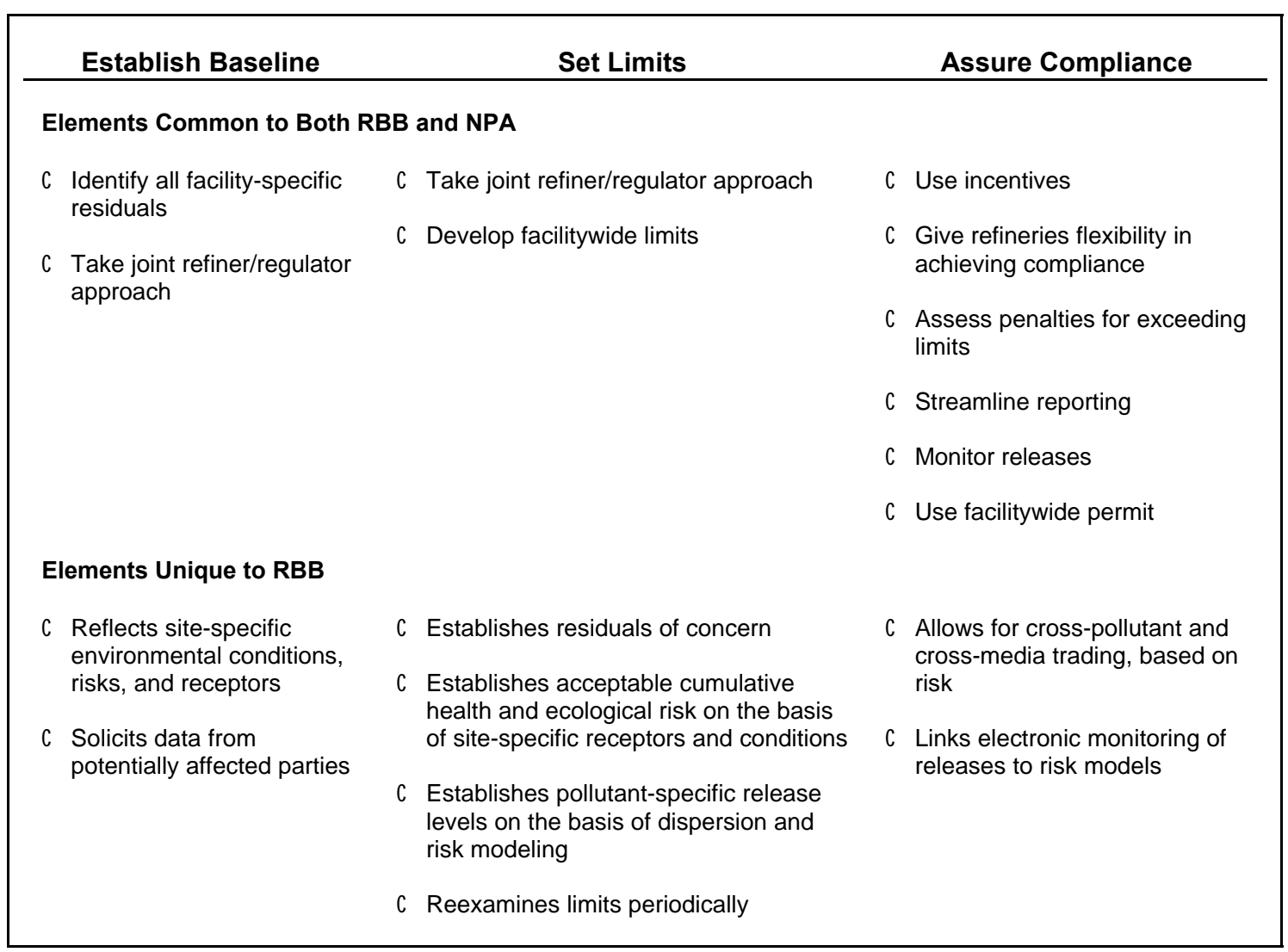


In the NPA strawman approach, the refinery and the regulator negotiate residual release limits on the basis of the inventory of actual residuals, current regulatory requirements, and anticipated regulatory requirements as stated in applicable federal laws and projected in the EPA's semiannual regulatory agenda. The limits are set to achieve a specified net reduction from current requirements. Public participation in setting the limits is a key element of the NPA strawman approach.

Compliance is assured via the environmental performance agreement, which contains release limits for specific pollutants, overall reduction goals, and compliance assurance requirements. This agreement also serves as a facilitywide permit. The terms of the NPA remain in force for a specified period of time. Progress in reducing releases is measured against the baseline. If limits or reduction goals are not met, affected interests (e.g., local citizens) are compensated. If disputes arise on any aspects of the NPA, a predesignated arbitration panel hears and rules on them. Table 3 summarizes key elements of the NPA strawman approach.

Table 3 Key Elements of the Negotiated Performance Agreement Strawman Approach

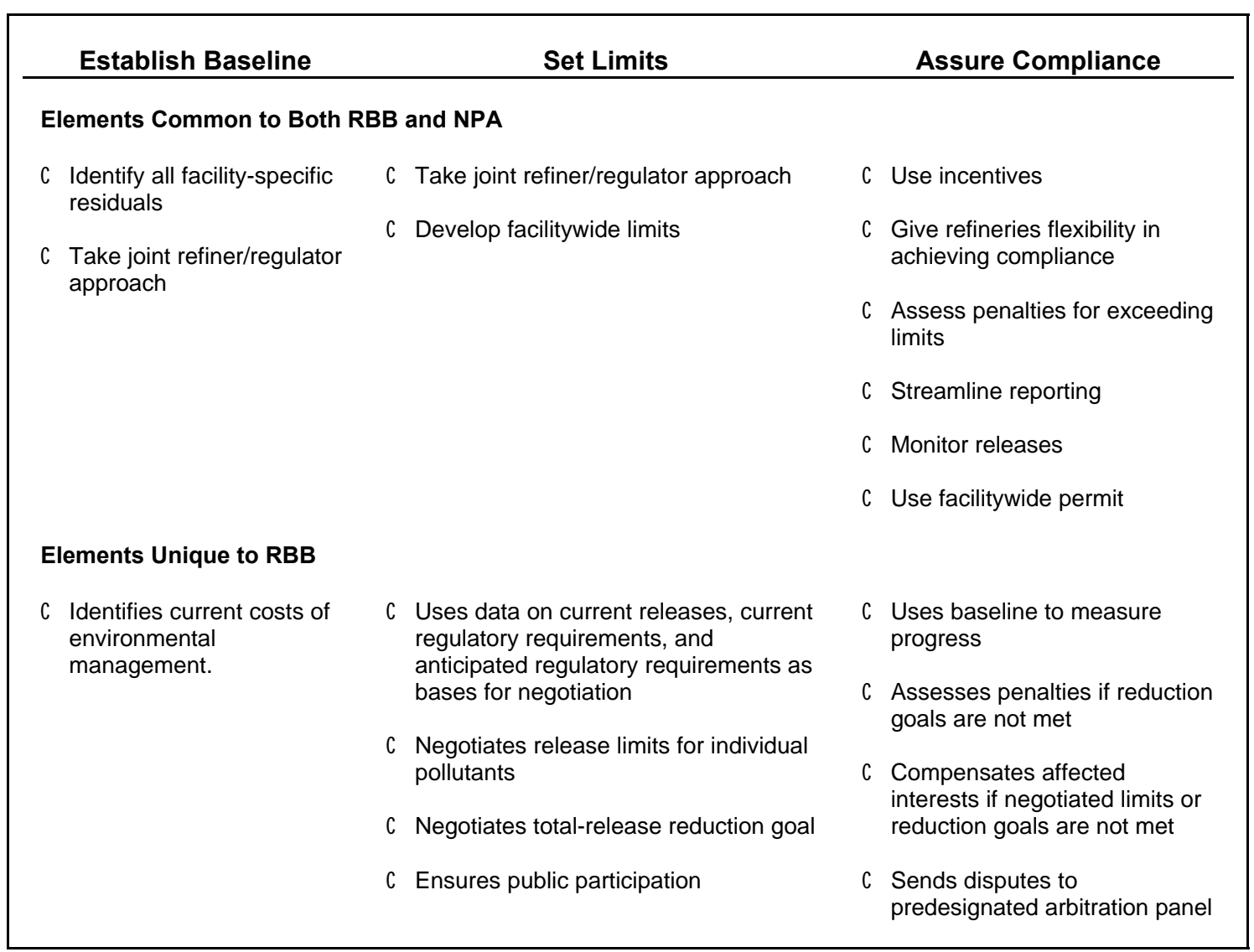




\section{Section 4 \\ Workshops}

The strawman approaches contain elements significantly different from those of existing regulatory programs, and implementation could fundamentally change the operating procedures of both refiners and regulators. The project team thus deemed it necessary to obtain input and feedback from interested parties early in the approach development process. After drafting the strawman approaches, the team conducted a series of workshops with representatives of various potentially affected parties. To promote candid dialogue and useful input, small, one-day workshops, each with participation by representatives from a single interest group, were planned. Seven workshops were held with the following stakeholder groups:

1. Petroleum refineries and trade associations,

2. EPA headquarters offices,

3. Texas and Louisiana state regulatory representatives,

4. Texas and Louisiana citizens groups,

5. National environmental groups,

6. Mid-Atlantic state regulatory representatives, and

7. Mid-Atlantic citizens groups.

To help ensure comparable results, the format for each workshop remained constant. Section 4.1 summarizes the workshop format, and Section 4.2 summarizes the workshop findings.

\subsection{Workshop Format}

The each workshop was conducted according to a consistent, structured format with the following components:

- Identification of and invitation to 6 to 10 representatives from a particular interest group. Representatives were identified on the basis of their previous contacts with project team members, perceived interest in regulatory reform, knowledge of refinery operations and environmental regulations, and knowledge of their organization's positions regarding reform efforts.

- Distribution of background reports and detailed agenda. Before each workshop, participants received background materials, including guidelines and principles, reports 
on laws and regulations applicable to refineries, and reports on the future operating environment for refineries.

- Presentations summarizing background information and draft approaches. At each workshop, the previously distributed background materials were summarized, and participants were asked to comment on the adequacy and accuracy of the information. The structures and concepts of the two draft approaches, duly noted as preliminary strawman approaches, were then presented. Participants were asked to provide their perspective on elements they would prefer in the draft approaches.

- Exchange of information regarding the components and elements of the two draft approaches. The focus of each workshop was a facilitated exchange of ideas among participants. Participants shared their concerns, criticisms, suggestions, and other comments on the overall three-component structure and on the elements of the RBB and the NPA.

- Summary of findings. At the end of each workshop, the project team summarized what it had heard, asked participants whether they agreed with the conclusions, and asked them to provide any additional comments or data they believed would be of use in developing the approaches.

\subsection{Workshop Findings}

Each workshop elicited constructive criticism, candid observations, and thoughtful suggestions for improving the approaches. A particularly noteworthy finding was that while differences were expected among the various stakeholder groups, the differences expressed by participants in an individual workshop often diverged as much as, if not more than, the differences expressed by participants in different workshops. For example, according to representatives at the refinery/trade association workshop, refiners do not have a common position on regulatory reform. Some seek major changes, some seek relatively minor changes to improve specific aspects of the regulatory system, and some prefer maintaining the status quo. In general, therefore, specific issues and concerns can rarely be attributed to specific stakeholder groups; thus, the team made no attempt to link comment type with stakeholder type.

The following sections describe workshop findings. Section 4.2.1 highlights comments commonly expressed in all workshops and applicable to both approaches. Section 4.2.2 summarizes comments pertaining to the RBB, and Section 4.2.3 describes comments regarding the NPA. Appendix E lists comments, organized by area of concern, expressed by one or more workshop participants; thus, it includes comments heard repeatedly as well as comments that may have been expressed by only one or a few participants. (Because of the frequent overlap of concerns among the various groups, the organization of comments by area of concern was deemed to be more efficient than by stakeholder group.) 


\subsubsection{General Comments}

\subsubsection{Current Regulatory Approaches Need Improvement}

Most participants agreed that the current environmental regulatory system needs to be improved. Despite initial skepticism in some cases, most workshop participants supported the project team's endeavors to develop alternative regulatory approaches for the petroleum industry. However, opinions varied regarding the degree and nature of required change, and several participants stressed that they would need to see more details before they could endorse or oppose specific elements or approaches. For example, most refinery representatives agreed that the costs of the current regulatory system were high and wanted to see reductions in such costs. However, they said they needed more information before they could evaluate how the strawman approaches would reduce those costs. Some expressed concern regarding anything new, noting that previous attempts to "fix" environmental regulatory problems resulted in increased costs. They also expressed concern about the resources required to implement these approaches, noting that small, independent refiners often prefer "command-and-control" approaches because they lack the staff to implement the types of changes envisioned by the alternative approaches. However, some participants enthusiastically endorsed the ideas embodied in the alternative approaches and explained that they were actively pursuing some of the elements contained in the strawman approaches.

Many local citizens group representatives said that the current regulatory system was unsatisfactory because, from their perspective, environmental and health conditions around refineries needed improvement. They also said that citizens should be more involved in refinery activities that can damage human health or the environment and that they would oppose any approach that would allow environmental conditions to deteriorate. They cautioned that sometimes increased flexibility - a key aspect in both approaches — can result in poorer environmental conditions, especially in the short term. They noted that such situations can occur when a company is offered a delay in compliance in exchange for an agreement to provide even greater environmental improvement at a future date.

Several participants noted that even though a facility may be complying with the current regulations, it may be releasing emissions that negatively impact the environment. However, citizens and regulators have no recourse, because technically the facility may be operating according to its permit. Some national environmental group representatives said that the current regulations governing petroleum refinery operations were neither sufficiently comprehensive nor transparent.

While most participants agreed that change was required, some state regulatory representatives argued that the current system provided sufficient flexibility, and that rather than replacing the current system, steps should be taken to improve the current system. Some refiners suggested that the approaches should be presented as alternatives to, rather than substitutes for, the current system, allowing for choice among various options. Several participants commented that the refinery should match any increased flexibility with increased accountability to the regulator and to the public. 


\subsubsection{Meaningful Public Participation Is Important throughout the Process}

Most workshop participants stressed that public participation from local stakeholders should be integrated into each step of each approach. They noted that while the strawman approaches call for community input for certain elements (e.g., for the environmental characterization aspect of baseline establishment in the RBB), such involvement is important for all elements.

Most participants noted the importance of trust, and many linked increased trust to increased public participation. Significant distrust exists among various parties (citizens groups, state regulators, refineries, national environmental groups, EPA regulators), and any steps to increase trust would be welcome. Several participants noted that trust will grow as citizen involvement increases.

Although they agreed on the importance of stakeholder participation, several commenters expressed concern over the resources needed to support such participation. They suggested that while in the long run, the overall costs of regulatory development and implementation may decrease as trust increases, securing the additional resources needed to incorporate public participation in the early stages may be difficult.

An important component that would encourage public participation and trust is public education. Information on the significance of certain chemical emissions, transformations that occur after a pollutant is released, the role of risk assessment, and other technical issues will help stakeholders understand the tradeoffs from the existing system, focus on higher-priority concerns, and make informed decisions.

The commenters also noted a need for the independent verification of information provided by citizens groups and other groups. Some regulators said that local surveys can be biased or unauthenticated, and that university-sponsored studies need to be peer-reviewed.

Several participants asked about the nature and level of public participation. Questions included these: Who sits at the table? Who sets the goals? Will the public be involved in decision making? Rules for public participation that identify participants, describe the roles of the participants, and determine the extent of that participation must be defined. Representatives from national environmental groups noted that the personalities of the participants and their abilities to work together can significantly affect the outcomes of public participation activities.

\subsubsection{Environmental and Economic Goals Are Equally Important}

Several participants noted that the approaches should not favor economic goals over environmental goals, and that to be attractive, the approaches must provide both environmental and economic benefits. Some participants wanted assurance that the alternative approaches would actually provide environmental benefits and not just reduce costs of meeting current environmental regulatory requirements. Others observed that refineries would have to see clear economic benefits before they would accept alternative approaches. Noting that three fourths of capital investments made by the refining industry are used to meet environmental requirements, one refinery representative said that the key question for refiners is how to reduce overall costs. 
Refiners would be unlikely to adopt any alternative approach without first seeing a demonstration that such an approach would pay back the capital invested or significantly reduce compliance costs.

\subsubsection{Approaches Need Good Performance Indicators and Measures of Success}

At least two types of indicators are required. One set should evaluate the success of the alternative approaches. Participants suggested that these indicators could include overall time and effort reduction and community satisfaction. A second set, which could be considered a subset of the first, should measure changes in public health and environmental conditions. National environmental group participants said that indicators should reflect environmental cost accounting.

\subsubsection{Approaches Need More Details and Specifications}

Several participants said that while they endorsed the concepts embodied in the approaches, they needed more details to provide a critical evaluation. For example, one refinery

representative stressed that he would like to see how the options would be implemented and how research on new tools (e.g., site-specific risk assessment, measurement of results) would be linked to overall regulatory approaches. He noted that the development of different methodological tools (e.g., monitoring, risk assessment techniques) can be as important as developing a comprehensive approach. Some participants expressed concern over the use of the baseline, suggesting that if it were used as a permanent target, there would be little incentive for refiners to reduce releases below the baseline.

Many participants said that certain terms such as flexibility, pollution prevention, and better environmental performance needed clarification. Several participants asked how "streamlined reporting" would be defined. They noted that the environmental community wants information and that streamlined reporting could be interpreted as providing less information. Some local citizens group representatives suggested that streamlining should mean that data are reliable, accessible, meaningful, and transparent; data should be presented clearly, placed in context, and require minimal interpretation. Participants also noted the importance of defining what will be enforced and how enforcement will occur. Refiners said that clearly expressed limits facilitate compliance and minimize the potential for misunderstanding. Several representatives asked for additional information and examples of incentives and disincentives. Some regulators asked what the penalties would be if, despite good-faith negotiations, refiners exceeded the release limits.

\subsubsection{Implementation Issues Must Be Addressed}

Almost all participants observed that federal laws and regulations would have to be changed in order for either of the strawman approaches to be implemented. EPA headquarters

representatives said that they would want clear statutory authority to promote pilot studies. Some regulators questioned whether they had the expertise to set refinery-specific rather than sourcespecific emissions limits, and said that they did not want to be placed in a position of relying on information provided by the refiners. Almost all participants commented on the importance of 
electronic monitoring. Some refiners said that a key factor affecting the acceptability of the alternative approaches is the availability of suitable tools, particularly for monitoring and modeling of baseline and compliance activities. They noted that adequate monitoring and measuring tools can provide a "comfort blanket" to state regulators. Some state regulators said that electronic emissions monitoring embodies good science, helps increase trust, reduces the need for modeling resources, builds good public relations, and, over time, provides economic benefits for regulators and refiners. Citizens group representatives suggested that the participation of citizens in monitoring could benefit all parties and that cooperative efforts could help identify and solve problems. They also suggested increasing the numbers and locations of monitors. They emphasized that because of the chemical changes that occur as emissions leave the stack, monitoring of emissions in the community provides more useful data than monitoring emissions in the stacks.

\subsubsection{The Boundaries Set by the Guidelines and Principles May Limit the Success of the Approaches}

Several participants questioned the principle that the alternative approaches apply to refinery operations only and do not extend to exploration and production activities or to product end use. Some national environmental group representatives said that the guidelines and principles, and hence the approaches, may be too limiting to provide the kinds of results that they would want to see. They and some state regulators observed that many refiners are beginning to view themselves as providers of energy rather than of petroleum products, and that with increasing consolidation in the industry, many parent companies have broad interests, with refining representing only one piece of a larger portfolio. Refiners also noted that today, environmental requirements associated with end-use fuel specifications have a greater impact on capital investment decisions than refinery environmental requirements. As a result, refiners may be more interested in approaches that address product requirements instead of or in addition to refinery requirements. Some EPA headquarters representatives suggested considering the benefits of cleaner products versus changing refinery processes. Several participants also noted that the approaches should consider worker health and accidents in addition to public health and environmental concerns.

\subsubsection{Three-Component Structure Is Appropriate}

As described in Section 3.1, the project team drafted the two strawman approaches to be consistent with a structure that calls for establishing the baseline, setting the limits, and assuring compliance. In general, the workshop participants agreed with this approach. However, an EPA headquarters participant suggested adding a component at the beginning to establish the need and a component at the end to evaluate performance. One citizens group representative suggested adding a component at the end that would measure progress, and others suggested making "assure compliance" the fourth component, and adding a third component that would measure performance. Some national environmental group representatives suggested that the second component, setting limits, implies that the approaches were limited to meeting those limits and that once those limits were met, efforts to achieve further release reductions would not be necessary. They suggested that the second component should include setting goals as well as limits and that the goals should include credibility and public disclosure. They also said that the 


\section{$\Delta$}

"assuring compliance" component should include assuring accountability and that the components should include the identification of pollution prevention opportunities.

Some state regulatory participants suggested that the three components imply a static system in which progress is measured against a baseline. Regulators said that the system should allow for continuous improvement; i.e., continuous reductions in releases. Although refiners want certainty, the system must be able to incorporate and reflect changes in science that will occur over time. They suggested adding an arrow from the third component (assure compliance) back to the first component (establish baseline), which would provide a loop to help assure continuous improvement and indicate that the process is dynamic. They noted that with this loop, the threecomponent structure could be viewed as an environmental management system, which, they noted, could provide a sound foundation for alternative regulatory programs.

\subsubsection{Comments on the RBB}

Many of the comments regarding the RBB pertained to the need for more knowledge and understanding of specifically how risk would be used in the approaches. Many participants endorsed the concept of a risk-based approach, but most questioned how the RBB could be implemented, especially in the short term, given the number of technical questions that would need to be answered. For example, EPA headquarters representatives noted the need to understand possible synergistic effects among pollutants and how people and animals react differently to different environmental contaminants. They also noted that the EPA has been reluctant to embrace multimedia approaches because of knowledge gaps; when the EPA has promoted such concepts in the past, the public and others have attacked the scientific basis of such concepts and, in the process, undermined the credibility of the agency. Mid-Atlantic regulators noted that misperceptions about risk would need to be addressed. For example, they noted that many people assume that a risk of one in one million means that for every million people, one will die instead of one may die. To correct such misperceptions, the RBB would need to include a public education element or develop alternative measures of risk.

Lacking confidence in available data, some observed that implementing the RBB would require more and better information than is currently available. They suggested that the approach may be viable in the longer term, and taking incremental steps to begin implementing the RBB in the nearer term may "drive the science to make it happen." One participant noted that the RBB may help address an issue with the current standards; i.e., that existing environmental regulations "keep clean areas clean and dirty areas dirty." The RBB strives to keep all areas within acceptable risks.

\subsubsection{Establish Baseline}

- Residuals of concern. Given that risk-based limits would not be established, at least initially, for all of the residuals released from refinery operations, the approach would need to identify the residuals of concern for which limits would be set and prioritize those residuals. Participants generally agreed with this approach, but asked how priorities would be set and what criteria would be used to identify residuals of concern. 
- Public availability of baseline information. Several participants asked if information on the contextual meaning of residuals (e.g., toxicity, exposure routes, health effects, ecological effects) would be made available to the public and, if so, how. Texas and Louisiana state regulators noted that making such information available would increase understanding of how residuals of concern were selected, how the baseline was established, and what bases were used for setting appropriate risk-based limits.

- Characterization of environment. Several participants asked how information on residuals and nearby sources necessary for characterizing the environment surrounding the refinery would be obtained and used in the characterization. Texas and Louisiana regulators explained that characterizing and understanding local environmental conditions is not as scientific as many would hope. Typically, perceptions are built into such characterizations, and submission of contradictory data is likely. Mid-Atlantic citizens group representatives noted that various organizations (e.g., regulators) often ask them for information but then never use it. Thus, these representatives said they wanted assurance that the environmental characterization data solicited from them would be used.

- Information verification. Regulators cautioned that information should be viewed in the context of the credibility of the organization supplying the data, the ability of the data to sustain peer review, and the possibility that the news media could publish unsupported data. Given that information for characterizing the environment would be solicited from local citizens, workshop participants asked what kinds of quality assurance/quality control procedures would be used to select data sources and independently verify information.

\subsubsection{Set Limits}

- Citizen participation. Several participants felt that citizen participation should be required not only in establishing the baseline but also in setting limits and in assuring compliance. Texas and Louisiana state regulators said that it would be impossible to implement the RBB if citizens were allowed to participate in only one step and not in subsequent steps.

- Acceptable risk. Several participants asked how the acceptable risk levels would be determined. They wanted to know who would determine acceptable risk. They also wanted to know if all communities would use the same acceptable risk level or if different communities could establish different risk goals. Some citizens group representatives expressed concern that if acceptable risk levels varied from community to community, industry would move to those communities with the higher acceptable risk levels. Mid-Atlantic state regulators said that because of resource constraints, states should not bear the burden of determining acceptable risk limits; for consistency, one single federal agency should be responsible for establishing acceptable risks.

- Cumulative risk. Participants asked for a definition of cumulative risk. Mid-Atlantic citizens group representatives suggested that cumulative risk could mean different things. 
For example, it could refer to the effects of releases that enter multiple environmental media, or it could refer to multiple chemicals released simultaneously over time, or it could refer to multiple facilities releasing the same or different chemicals. Some participants noted that while the approach calls for the incorporation of nonrefinery releases in the calculation of cumulative risk, disagreements regarding the sources of those releases could affect actual implementation. Participants wanted to know what process would be used for working with other industries to establish risk-based limits. Some citizens groups said that cumulative effects should be considered in setting the limits even if the effects of those pollutants may not be experienced for 20 years.

- Chronic/acute risk. Some participants asked how the RBB would distinguish between acute risks and chronic risks.

- Data limitations. Texas and Louisiana citizens group representatives noted that it is often difficult to prove whether a chemical does or does not cause cancer. There is always a perception, perhaps based on previous studies, that a specific chemical is linked to a specific disease, even if the cause cannot be proven clinically. In some areas (e.g., Calcasieu County, Louisiana) where many health problems exist, it may be impossible to convince residents that refinery emissions did not cause, or at least contribute to, these health problems. Citizens group participants asked how the program would address the lack of certainty associated with linking pollutants to probabilities.

- Modeling and monitoring. Noting that risk models would be used to calculate the emissions limits, participants wanted to know the sources of data that would be used in the models, how such data would be obtained, and how modeling quality would be ensured. Citizens group representatives also expressed concern over the use of modeling to estimate pollutant concentrations and strongly supported using monitors rather than models. They also noted that the monitors should be placed where the people are, not at the fence line.

- Reopeners. Noting that the RBB allows for reexamination of the risk-based limits when new information could affect such limits, several participants asked what conditions would be required for such reopeners to occur (e.g., they asked if consensus would be required). Texas and Louisiana state regulators noted that reexamining limits in the light of new data was important, but that from a practical perspective, refiners may resist such reexaminations. They explained that if a plant manager has been operating under certain assumptions about the chemicals being controlled, introducing new information may require the manager to change refinery processes, leading him or her to view such changes as taking time and resources away from other activities. Similarly, they suggested that once a refinery operator has invested capital in a particular process to reduce a certain pollutant, the operator may feel that he or she has been misled if new data indicate that the pollutant has different effects than previously believed. If the pollutant is less harmful than originally believed, the refinery may "lose credit" for reductions of that pollutant. Alternatively, if the pollutant is found to be more harmful than originally believed, new equipment or processes may be required to respond to the new information. Refiners may prefer standards that are not subject to change. Citizens 
also may balk at reexamining limits, especially if new information suggests that a chemical may pose less risk than previously thought.

\subsubsection{Assure Compliance}

- Trading of releases. Some participants raised issues about provisions that would allow trading of releases of the same pollutant across different environmental media and allow trading of releases among different pollutants. Texas and Louisiana regulators noted that trading across media and pollutants can become complicated. Given the lack of knowledge about many compounds, the perception that some chemicals are dangerous regardless of the amount, and the negative publicity that could result from attempting to trade some of these chemicals, regulators suggested that the RBB, at least initially, prohibit the trading of certain pollutants. National environmental group representatives suggested that trading pollutants beyond the plant boundary should be limited to pollutants such as sulfur dioxide $\left(\mathrm{SO}_{2}\right)$ and nitrogen oxides $\left(\mathrm{NO}_{\mathrm{X}}\right)$ and that toxic pollutant trading raised questions about health impacts. Participants also asked the following questions:

- How would pollutants eligible for trading be identified; who would be responsible for deciding what chemicals would be traded?

- What tools would be developed and used to implement cross-media and crosspollutant trading?

- How would the initial allocations for trading be made for each pollutant for each refinery, particularly when other sources are nearby? (Several participants said that they viewed trading within a facility to be acceptable, but that trading across facilities raised concerns.)

- Would caps be set for pollutants traded across media, and, if so, how would those caps be established?

- How would the temporal aspects of trading be considered? (Some participants noted that trading seems to provide flexibility as a primary benefit, but environmental benefits are often secondary to the financial benefits.)

- Monitoring. Participants wanted more details on how and where monitoring would be conducted. Citizens groups stressed the need for monitors to be placed in strategic locations (e.g., near schools). They also suggested that citizens could be trained to operate the monitors, and that doing so might reduce costs and mitigate citizen distrust of contractor-operated monitors. Participants also asked how the public could obtain monitoring results, noting that such public availability would help assure compliance. 


\subsubsection{Comments on the NPA}

Most of the comments regarding the NPA sought additional explanation and clarification of the various elements in the three components. Virtually all participants agreed that public participation must occur in all NPA components. Some state regulators suggested that in the NPA, an initial component should be added that would set goals. Thus, before identifying current emissions, the goals of the program would be agreed upon. (They noted that this additional component would not be needed in the RBB, if one assumes that the acceptable risk levels, or goals, are determined by an outside authority.)

Several participants said that the NPA appears to require less change from current practice than does the RBB and that it shares characteristics with other regulatory reinvention approaches. For example, Mid-Atlantic state regulators noted similarities between the NPL and the plantwide applicability limit (PAL). A PAL is an emissions level established to limit allowable emissions of a source so that major new source reviews are not required for physical or operational changes to the facility as long as the total emissions from that facility remain under the PAL. Regulators suggested that the key difference between the PAL and the NPA is that the PAL pertains only to air pollutants, while the NPA considers all environmental media. One participant suggested that the PAL could be used as a "stepping stone" toward fuller implementation of the NPA. Also, national environmental group representatives suggested that the NPA was essentially a Project XL approach, and this seemed to be acceptable. As such, they noted that the NPA may be easier to implement than the RBB in the short term.

\subsubsection{Establish Baseline}

- Ranking of residuals. A common comment pertained to whether all releases, regardless of pollutant, would be treated in the same way. Participants wanted to know whether refinery residuals, and thus their releases, would be ranked in some way (e.g., related to human health effects) or whether they would all receive equal weight. Some participants suggested that the NPA should reflect the concept that some residuals are more potent than others, and others suggested that the residuals be placed in some kind of context, such as how they affect human health or how they compare with previous releases.

- Baseline definition. Several participants asked whether the baseline would reflect current operating experience or current regulations. Some national environmental group representatives suggested that using current regulations would be unsatisfactory, because current regulations are not sufficiently comprehensive or transparent. Several participants also noted that all residuals (i.e., those that are currently regulated and those that are not) should be included in the baseline.

- Pollution prevention. Some state regulators suggested that in addition to identifying residuals and costs, the baseline should also identify opportunities for pollution prevention. 
- "Gaming” the baseline. Several participants asked what controls would be used to prevent refineries from setting the baseline lower than it actually is in order to show progress relative to that baseline.

- Baseline as a target. Some regulators expressed concern that the baseline would be a static target, thereby limiting potential environmental improvements to the baseline and not allowing for improvement beyond the levels set in the baseline. They said that the approaches needed to provide a means for continuous improvement.

- Public participation. Several participants commented that the public should be involved in establishing the baseline. Most regulators said that local citizens groups as well as the refiners and the regulators need to be involved in establishing the baseline. Citizens groups noted that community participation should involve community residents, not just elected officials or community advisors appointed by the refinery.

- Costs in the baseline. Several participants questioned the role of costs in the baseline. Some national environmental group representatives asked for a clear explanation of how costs would be defined and how the identification of costs would relate to the negotiated agreement. Some state regulators asked how the approach would protect the confidentiality of cost information, and Mid-Atlantic regulators noted that clear rules should govern confidentiality of information. Several participants noted that costs should reflect economic externalities (e.g., changes in property and land use values) as well as environmental management costs and that environmental management costs should include monitoring as well as capital costs. Mid-Atlantic regulators expressed concern that refineries might use past capital expenditures to argue against new environmental regulations. Others recommended using total cost accounting or life-cycle approaches to identify costs.

- Source identification. Texas and Louisiana state regulators suggested that sources of releases should be identified. Even though limits would be set on a facilitywide rather than a source-specific basis, knowing the sources of the releases would help in identifying causes of such releases and might help in developing means to reduce those releases.

- Data validation. Mid-Atlantic regulators expressed concern that in establishing a baseline, refineries might overstate both residuals and environmental costs, and that regulators do not have the resources to verify the residual or cost information provided by refineries.

\subsubsection{Set Limits}

- Pubic participation. Several participants asked who would be involved in the negotiations, and many emphasized that in addition to the refinery and the regulators, the public needed to be involved. Texas and Louisiana citizens groups noted that community representatives would be at a disadvantage because they are less knowledgeable, and "knowledge is power." They suggested using technical assistance grants to educate 
citizen participants. They noted that many citizens are already somewhat knowledgeable in environmental areas, and that the environmental movement and increased awareness are making it easier to educate community representatives. Other participants asked how stakeholders would be selected and who would determine which stakeholders would participate. They asked for details on the process of including the public in the negotiations.

- Roles of the negotiators. Many participants said that the approach needs to specifically define the parties that would participate in the negotiations. State regulators asked if all those "at the table" would be involved in the negotiations and what roles elected officials would play. They also asked what processes would be used for contesting the limits.

- Information dissemination. In addition to identifying the stakeholders, several participants said that the approach needs to consider and define who would receive the information generated as the limits were set.

- Reaching agreement. State regulators asked how agreement would be reached. While several approaches are possible (e.g., consensus, majority), participants would need to know up-front when agreement had been reached.

- Early reductions. Several participants asked how, in setting the limits, the NPA would consider reductions in releases made by the refinery before the baseline was established.

- Implications of using anticipated regulations. Noting that the NPA limits would be set on the basis of existing and anticipated regulations, some participants asked what would happen when the promulgated regulations were more stringent than the limits.

- Term of agreement. Several representatives suggested that the life of the NPA be defined.

- Measurement units. Some participants mentioned that the NPA needed to ensure that the measurement units used for the release limits reflect the impacts they were intended to address. For example, they noted that reductions defined in tons per year might still allow significant short-term adverse impacts and, therefore, might not be appropriate.

- Flexibility. The NPA would be in force for several years. State regulators noted that provisions need to account for changes in production and environmental conditions that could increase releases or make goals otherwise inappropriate. State regulators also asked how flexibility could be built into an agreement that designed to last for several years.

\subsubsection{Assure Compliance}

- Milestones. Noting that the NPA would provide the refinery with a specified time frame (in years) in which to meet the required reductions, several participants asked what, if any, compliance milestones the refinery would have to meet during the course of the 
agreement. Mid-Atlantic regulators noted that communities are much more interested in day-to-day refinery emissions than in long-term limits. Texas and Louisiana state regulators explained that if milestones were not required, citizens might not accept the program, because they would be afraid that at the end of the period, the refinery might not reach the goals. Regulators questioned whether they would have the resources to enforce the milestones.

- Penalties. State regulators asked what would happen if, at the end of the performance period, the goals had not been met. They also said that the timing of penalties needed to be specified. They asked if penalties could be assessed at any point in time during the NPA, or only at the end of the agreement period. Assuming penalties could be assessed during the interim, they asked what process would be used to determine the kinds of penalties that would be assessed and under what conditions.

- Measurements against the baseline. State regulators asked how releases would be measured against the baseline. They emphasized the amount of skepticism that exists over the use of calculated estimates versus measured values. Calculated estimates lead to distrust; community groups feel that refineries "fudge the numbers" and "play games" with results. A system that would use actual monitoring results would be more likely to be accepted than one that would rely on calculated estimates.

- Failure to meet goals. Texas and Louisiana citizens groups asked how the NPA would control for the possibility that the goals would be set too high and therefore not be met. They asked how the refineries would be penalized in such cases.

- Compensating affected interests. Several participants asked for specific examples illustrating how affected interests would be defined and how they would be compensated if refineries failed to meet the goals.

- Dispute resolution. Mid-Atlantic regulators suggested considering alternative ways to resolve disputes besides the proposed arbitration panel. They said that while common ground may be reached through use of an arbitration panel, the common ground reached may not be protective of human health.

- Information dissemination. Citizens groups in Texas and Louisiana said that the NPA needs to place more explicit emphasis on information management. The community has a strong desire for access to reports. Acknowledging that raw data may not be understood, they suggested that an interpretation sheet accompany all reports.

- Liability. Some national environmental group representatives suggested adding liability as an element for assuring compliance and accountability.

\subsection{Using the Findings to Revise the Approaches}

As described in Section 3, the project team developed two draft strawman approaches as a starting point for discussion with potentially interested parties. The strawman approaches were 


\section{$\Delta$}

drafted to meet the goals of environmental responsibility, economic performance, and pollution prevention technology development and use, and to be consistent with the guidelines and principles described in Section 2. Feedback obtained from the seven workshops was used to expand and revise the approaches.

Because most of the workshop comments stressed the need for more information and clarification, and few specifically endorsed or rejected individual elements, the team combined the workshop comments with results from studies and reports on regulatory reinvention and risk assessment to provide additional details. Many of the workshop comments applied to both the RBB and NPA, and both revised approaches address many of the issues and concerns issues in the same way.

The team tried to incorporate as many suggestions into the revised approaches as possible. Nonetheless, responses to two categories of questions were deferred. The first category of questions asked for details on setting risk-based limits. The project team, recognizing that risk assessment concepts, data, and understanding have grown significantly in the past 20 years, believes that such data and understanding will continue to increase during the next several years. Therefore, some of the risk-related questions raised in the workshops may be able to be answered better over the next several years. Because these alternative approaches are future-oriented and not expected to be implemented in the near term, deferring a response is consistent with the scope and concept of the process being used to develop alternative approaches. Second, some questions pertaining to the negotiated agreement in the NPA were deferred because they should be addressed during the actual negotiations. For these types of questions (e.g., length of term for performance agreement), the project team offers suggestions but recognizes that situationspecific needs will dictate the results of individual negotiations. 


\section{Section 5 Revised RBB}

As described in Section 4.2.2, many workshop participants endorsed the concept of the $\mathrm{RBB}$, but, upon considering the amount of data needed to set risk-based limits and the state of knowledge regarding risk assessments, they raised questions about implementation. They also asked for clarification and details about concepts such as acceptable risk, cumulative risk, and risk-based trading of pollutants. Most participants stressed the need for public participation in all components.

The project team acknowledges that these issues are not trivial. However, given the number of existing and proposed efforts to expand risk-related data collection and the increasing number of government and nongovernment organizations calling for increased emphasis on incorporating risk in regulatory reform efforts, the project team believes that these concerns will be resolved to that extent that the revised RBB could be implemented, at least on a pilot scale, within the next 15 to 20 years. This section describes the revised RBB, which reflects the input obtained during the workshops. It also identities ongoing research activities and related efforts that could facilitate application of the RBB.

\subsection{Overview}

The RBB defines allowable releases of pollutants from the refinery such that the total risk resulting from all releases and all sources, when considered in the context of the surrounding community, does not exceed a predetermined, acceptable, total cumulative risk level. Thus, several site-specific factors must be considered to set the release limits. These include the types of pollutants released from the refinery (whether currently regulated or reported or not), individual pollutant toxicities (to human and ecological receptors), exposure pathways, and exposed populations (including sensitive populations and populations who are located far away from the refinery but may be affected by pollutants that are transported over long distances). The RBB treats refinery operations as though a bubble surrounded the plant, with source-specific releases coming from the bubble rather than from individual stacks and other refinery sources. The approach considers the synergistic and cumulative effects of pollutants released from the refinery and from nearby facilities on public health and the environment. Because the controlling factor is total risk, pollutant-specific release rates can be modified as long as the total risks from all pollutants and the individual risks from specific pollutants remain within acceptable levels. The approach gives plant managers flexibility in controlling releases from disparate sources, as long as the total cumulative risk remains acceptable.

The RBB incorporates three key features. First, it directly links release limits to public health and environmental protection. Because these links consider toxicity, exposure routes, and affected populations, they ensure that reduced releases will result in improved public and environmental health. Second, because the target is expressed in terms of overall risk, refineries do not have to use specific technologies or target specific pollutants. Thus, the approach provides 
incentives to develop and implement new pollution prevention and reduction approaches. Third, the approach embodies public participation and communication provisions to promote understanding and acceptance by all affected parties. These features differentiate the RBB from the current system, and, as such, provide for meeting the goals of environmental responsibility, economic performance, and pollution prevention technology development and use.

The revised RBB approach comports with other efforts to improve regulatory decision making. For example, the revised RBB shares many elements with the draft findings of the Integrated Risk Project (IRP), a recent major undertaking of the EPA's Science Advisory Board $(\mathrm{SAB})$. The SAB initiated the IRP in response to requests from the EPA and Congress to develop a science-based approach to integrated decision making, considering environmental risks and risk-reduction strategies. SAB's draft summary report states that "scientific understanding of the causes and effects of environmental risk, including the interrelated effects on humans and ecosystems from multiple stressors, has improved substantially in the past decade" and that "those advances can help improve environmental decision making" (EPA 1999e). Several of the SAB's draft findings support the RBB approach. These include the following:

- It is likely that the greatest remaining threats to the environment and public health involve multiple stressors, sources, endpoints, and routes of exposure. Therefore, the greatest opportunities for risk reduction need to be sought by means of an integrated examination of risks.

- Risk management strategies can be tailored to specific communities to reduce multiple risks to both human health and ecosystems simultaneously.

- The next generation of environmental decision making will focus on demonstrated improvements in the environment resulting from integrated action rather than on a simple, single-pollutant strategy that infers, but does not demonstrate, a connection between process-based compliance and environmental improvements.

- The best strategy is not necessarily the one that reduces the worst risk to a de minimis level, but the one that reduces the most risk with the resources available.

- To incorporate qualitative and quantitative values information, formal deliberation among stakeholders can be useful in raising and defining qualitative issues and in assuring that quantitative issues are fully and properly included.

- Focusing on goals defined in terms of improved human health and ecosystem outcomes, rather than simply documenting steps taken, is consistent with the accountability mandates of the Government Performance and Results Act (GPRA).

- Transition to an integrated approach will require changes in the types and amounts of information collected, the kinds of analyses used to support decision making, the specific environmental goals to be achieved, and the ways in which progress is measured. 
- Decision-making procedures should make clear to the public the reasoning behind environmental decisions and include a stronger system of public accountability. Complete communication among those involved assures that all activities are coordinated and complementary.

Appendix F highlights several recent studies, including the IRP, which share common themes and concepts with the RBB.

The project team recognizes that detailed methodologies to translate pollutant interactions and resulting risk into release limits may not be currently available. However, the growing body of risk information that various organizations ${ }^{4}$ are developing should continue during the next several years, and scientific advances in the areas of chemical interactions and human and ecological effects should hasten the development of acceptable risk-based methodologies. In the interim, refiners, regulators, and other stakeholders can begin work on several RBB elements, so that once technical and other experts resolve scientific issues, program participants will be ready to implement the approach. The following subsections explain the elements that make up the revised RBB. Because the revised RBB requires stakeholder involvement in all components, and because the same or similar stakeholder provisions apply to each component, the first subsection describes the stakeholder involvement/public participation process, which is integrated throughout the revised RBB. The remaining subsections discuss the decision-making body (the RBB Board) and the three components (establish baseline, set limits, and assure compliance).

\subsection{Public Participation}

The RBB strawman approach required input from the local community to characterize the environment surrounding the refinery. It also provided that limits could be reviewed upon petition by refiners, regulators, or local citizens groups. However, it did not explicitly provide for public participation in the other components. Workshop participants and other recent studies have stressed the need for meaningful participation in all phases of regulatory processes. For example, the Presidential/Congressional Commission on Risk Assessment and Risk Management found that decisions based on public participation are "more durable, effective, acceptable, and implementable" (Presidential Commission 1997). Thus, the revised RBB calls for community involvement in all three components.

The revised RBB envisions two types of public participation. The first would involve local community members who are committed to taking long-term, active roles in implementing the

4 Examples of this growing body include (1) the Agency for Toxic Substances and Disease Registry (ATSDR), in its development of minimum risk levels for 280 hazardous substances; (2) the EPA's Office of Prevention, Pesticides, and Toxic Substances (OPPT), in its recently released risk screening environmental indicators, which combine toxicity data with exposure data for more than 500 chemicals and 38,000 sites to provide quantative indicators of relative risk; (3) the EPA Office of Air Quality Planning and Standards (OQAQPS), in its Report to Congress, which identifies current risk assessment techniques, data sources, and data gaps; (4) an August 1999 proposal that requires chemical manufacturers to provide exposure data for the EPA's Chemical Substances Inventory; and (5) various technology developments in the areas of open-path and fence-line monitoring. 
three components. These participants should represent broad community values, preferences, and concerns and should be selected by the communities they represent. These local community representatives would work together with representatives of the refinery and the regulatory agencies to implement the revised RBB on a refinery-specific basis. The second type of public participation is broader and would probably involve participants who are interested in sharing information but unwilling or unable to devote the necessary time and energy to the entire RBB process. These participants could offer data or information, raise issues for consideration, communicate results, and review data and other materials used in the decision making process. However, they would not have a direct role in decision making. The revised RBB recognizes the need for this level of public participation. It helps to ensure that all interests are considered, thereby reducing the chances of future challenges to decisions (e.g., on baselines, limits, penalty assessments). It helps ensure objectivity, since the public includes community groups as well as industry and regulatory representatives.

\subsection{RBB Board}

The RBB requires a decision-making body, which, for discussion purposes, is referred to here as the RBB Board. The size of the RBB Board should be large enough to represent the views of all stakeholders and small enough to operate efficiently and effectively. While the actual structure and composition of the RBB Board would depend on refinery-specific conditions, one possible format would consist of six members: two each from the refinery, the regulatory body, and the local citizens groups. These six individuals would constitute the decision-making body. As such, they would be responsible for agreeing on the baseline, approving the risk-based limits, and approving the compliance assurance system. All representatives would have to be flexible and willing to negotiate. They would also have to be knowledgeable or willing to learn about refinery, health, ecological, and risk issues. Workshop participants stressed that while participation should be broad (it should include all stakeholders who have an interest), careful selection of the participants who actually "sit at the table" is critical. The personalities of the participants and their abilities to work together can significantly affect the outcome of public participation activities. One possible option is to have a separate committee identify criteria for and select RBB Board members. Criteria could include an individual's credibility (within the group he or she represents), knowledge (or willingness to obtain necessary knowledge), negotiating and problem solving skills, commitment, and ability to be a collaborative group member. Facilitators could help identify, recruit, and interview potential participants.

Because the revised RBB requires the collection and analysis of significant amounts of data combined with an understanding of environmental and refinery processes, none of the RBB Board members could be expected to know all the information necessary to make the kinds of informed decisions that this approach requires. For example, because the current regulatory system reflects a single-medium approach that results in several regulatory agencies having authority over a single refinery, representatives of various regulatory bodies would need to exchange information and share it with the RBB Board. Similarly, different citizens groups have different areas of concern and expertise, which the RBB must reflect. Finally, because the RBB integrates pollution control measures into refinery processes, the plant manager may need to be a Board member, while relying on input from various process and environmental engineers. 
Therefore, the second type of public participation would enhance RBB operations by promoting the sharing of information in meetings, communication of results, solicitation of data, and consultations with and for the RBB Board. The RBB Board would use the information from the second type of public participation as input for its decision-making process, which would help keep Board resource demands to manageable levels.

The project team envisions a meeting at the beginning of the process in which the RBB Board would identify priorities, specify schedules, develop ideas on how technical and nontechnical information would be exchanged, and set ground rules. In recognition of the fact that the nature and degree of RBB Board workings would vary, depending on the refinery and the specific phase or element of the process, key facets of possible RBB Board operations, including public participation in those operations, are summarized below.

- Ground rules. Establishing ground rules will facilitate the smooth operation of the public participation process. Examples of the types of ground rules that should be considered include the following:

- Set objectives and goals (e.g., agree on baseline, agree on acceptable risk, develop release limits that allow for flexibility and accountability, and develop measures to indicate success).

- Develop schedules and milestones.

- Agree on communications outside the operating meetings (e.g., posting on the Internet, establishing library, managing confidential information).

- Define the decision-making process (e.g., consensus, majority, who makes the decisions). One option could be to enact decisions if four of the six RBB Board members vote to do so.

- Provide for membership changes (e.g., consider establishing terms to ensure continuity and rotations to encourage fresh ideas and limit burnout).

- Determine how meetings and decisions will be documented or recorded.

- Meaningful public participation. Public participation is time consuming. Most citizens interested in working with regulators and industry are volunteers, with a variety of commitments, often including participation on more than one board or committee. Their time is limited. Similarly, regulators and refiners must attend to a variety of regulatory and business functions, and finding time to work with the community may be difficult. Therefore, all time investments must be productive. Trying to explain to stakeholders why certain actions were taken after the fact, rather than exchanging information and ideas prior to taking action, is not productive. Public hearings where comments offered by stakeholders are not considered and carefully evaluated by a decision-making body are not productive. Public participation must be meaningful, broad, and continuous. 
Knowledgeable representatives. RBB Board members must be knowledgeable or willing to become knowledgeable and committed to reaching agreement. In the workshops with local community groups, the project team found that local community representatives understand the issues and are willing to learn more in order to participate effectively in discussions. The team suggests that refineries, regulators, or interested nongovernmental organizations provide training resources to educate citizens group representatives in technical areas such as health, risk, and ecological concerns so that all RBB Board members and as many other stakeholders as possible work from a common knowledge base. Possible training options include workshops, seminars, and Internet-based training. Public participation should provide a learning opportunity for all involved. In the long run, resources to provide training will be well spent. Similarly, regulators and refiners need to understand the concerns of the other parties involved; the public brings local knowledge and insights on factors contributing to risk that are otherwise unavailable.

- Use of facilitators. The RBB Board may wish to consider using a facilitator at its own meetings and should use a facilitator whenever it opens the meetings to outside participants. The additional cost for a professional skilled in eliciting views, building consensus, and resolving disputes is expected to pay off in more efficient allocation of time resources and better communication, which will reduce potential future conflicts and misunderstandings. The RBB Board may also consider conducting meetings on neutral ground, alternating meeting times, and providing day care and transportation.

- Role of consensus. Ideally, all participants will agree on baselines, release limits, compliance assurance methods, and performance indicators needed in the RBB. However, such consensus may be elusive. The project team believes that as long as all quantitative and qualitative issues have been fairly expressed and considered, the RBB Board can reach a decision without consensus, if the reasons for the decision are clearly explained. Disagreement and controversy are to be expected. The important issue is that all views are carefully considered.

Various organizations have reported on successful and unsuccessful public participation approaches. ${ }^{5}$ The RBB Board may wish to draw on the findings of such studies to help optimize decision-making processes. The following sections describe the revised components of the RBB, which the RBB Board will implement.

\subsection{Establish the Revised RBB Baseline}

As does the NPA, the RBB establishes a site-specific baseline for each refinery. However, while the NPA baseline considers refinery releases and costs, the RBB baseline considers refinery releases, the environment into which pollutants releases enter, and the characteristics of those pollutants that affect public health and ecology. Whereas the NPA baseline serves as a benchmark against which future releases can be compared, the purpose of the RBB inventory is

5 For example, OPTT has developed a Constructive Engagement Resource Guide, which draws on the experiences of representatives from the computers and electronic industry as they participated with the public in the Common Sense Initiative (EPA 1999a). 
to provide information to develop the initial refinery-specific release limits. Although these limits can change, as long as the risks associated with the release remain within acceptable levels, the baseline provides an initial starting point. The inventory also facilitates pollution prevention by providing release data to help target pollution prevention activities. The refinery, the regulatory body, and the community participate in establishing the baseline through the RBB Board.

To establish the RBB baseline, the RBB Board should direct five separate activities, each of which is listed below and described in the following paragraphs.

- Develop an inventory of refinery releases,

- Characterize the environment that is affected by releases,

- Characterize the pollutants of potential concern,

- Screen and prioritize the pollutants for setting release limits, and

- Document and communicate results.

\subsubsection{Develop Inventory of Refinery Releases}

Refinery releases can increase the risk of harm to human health and the environment. To identify optimal means for limiting these releases and to ensure that the total risks presented to public health and the environment are not unacceptable, the releases must be identified and characterized. This characterization entails a source-by-source identification of all releases from the refinery system (including leaks and fugitive emissions.) Findings of the 1991 Amoco/EPA Pollution Prevention Project in Yorktown, Virginia, show why all residuals - not just those currently regulated or reported - need to be identified (Klee and Podar 1992). In this project, the Amoco/EPA team first identified and assessed releases from the refinery and then developed and evaluated options to reduce those releases. The comprehensive release inventory, which included chemical type, quantity, source, and medium of release, correlated poorly with the releases measured for regulatory purposes. For example, the Toxic Release Inventory (TRI), commonly used to measure releases from reporting industries, covered only $9 \%$ of the total hydrocarbons released by the Yorktown refinery and only $2.4 \%$ of all releases to all media. (The TRI does not require reporting of criteria pollutants such as carbon monoxide [CO], $\mathrm{NO}_{\mathrm{X}}$, or $\mathrm{SO}_{2}$.) Similarly, the EPA does not require record keeping for certain activities and emissions sources. At the Yorktown refinery, emissions from barge loading made up roughly $20 \%$ of total benzene emissions, but the EPA did not require record keeping for such emissions (Klee and Podar 1992).

Establishing this all-encompassing inventory may be time consuming and costly. (The analysis of 15 to 20 chemicals from roughly 1,000 samples at the Yorktown project cost about $\$ 1$ million and required a year to complete.) Although costs may be high, workshop participants generally agreed that a comprehensive inventory is necessary to identify and understand the sources of risk and to identify cost-effective means to reduce unacceptable risks. The Yorktown 
study showed that about $95 \%$ of the release reductions required by regulatory programs could be achieved for $20 \%$ to $25 \%$ of the actual costs to the refinery of those regulatory programs (Klee and Podar 1992).

The process for identifying releases could be modeled on the Yorktown study by using the lessons learned from that approach and incorporating stakeholder input. Uncertainties and data gaps should be documented for later verification or investigation.

\subsubsection{Characterize Environment Affected by Releases}

The risks associated with pollutant releases from refineries depend not only on the quantities of chemicals released but also on the site-specific hydrogeologic and climatic conditions and the population characteristics of the environment into which they are released. Thus, information about the environmental conditions within and surrounding the bubble (the area of potential impact) must be characterized. Such characterization entails a detailed identification and mapping of the local conditions that can affect the likelihood that specific refinery releases will translate into health or ecological risks. Information needed to characterize the baseline includes, but is not limited to, the following:

- Locations of populations, including sensitive populations (e.g., children, elderly), schools, day care centers, hospitals, and nursing homes, relative to the refinery;

- Water bodies and associated watershed areas and flow rates, including tributaries that may be affected by refinery releases;

- Wind speed and direction - both local and long-range - to identify potential risk areas that are located away from the source (e.g., risks from long-range transport of $\mathrm{SO}_{2}$ );

- Sources of drinking water and potential pathways of releases to that water;

- Current and reasonably anticipated land uses;

- Buildings, terrain, and other geographical features that may affect dispersion of air pollutants;

- Other polluting sources in or near the community, the releases from which could interact with those of the refinery and affect public health or the environment;

- Potential exposure scenarios;

- Plant types; and

- Soil characteristics.

For most releases, the affected communities will be those near the refinery. However, for pollutants that can be transported or transformed over long distances (e.g., $\mathrm{SO}_{2}$ ), affected 
communities may be far from the refinery. Thus, potential exposure data must be obtained not only from citizens near the refinery but also from regulators or other organizations that can address the potential impacts of refinery releases on distant populations. The RBB Board should seek assistance from organizations (e.g., regulatory bodies in other jurisdictions) to assist in estimating the fate and transport of ozone and acid rain precursors over long distances and in identifying potential receptors (including significant ecological receptors) in these areas.

Much of the information needed to characterize the environment is currently available. For example, U.S. Geological Survey topographical maps, maps of land use and land cover, and aerial photographs can provide initial data. The local community should verify this input. Peerreviewed surveys or studies conducted by local universities could also supplement basic information. The RBB Board should provide for the evaluation of data and should ensure that all information used in the characterization is referenced. Sources of uncertainty and discrepancy should also be documented, and data gaps should be indicated. Such documentation will help answer questions that could arise in the future and can help direct future research activities.

The role of public participation in characterizing the environment cannot be overemphasized. Local community members can identify specific exposure conditions in particular parts of the community and populations subject to increased risk. Such information can help in setting priorities when release limits are being established and may also help to identify more effective nonrefinery solutions for lowering the risks of specific receptors (e.g, the installation of air purifying devices in the home.)

Once the environment is characterized, the RBB Board should identify the significance and contribution of various exposure pathways. An exposure pathway consists of a release source, release mechanism (e.g., fugitive emissions), transport medium (e.g., air), exposure point (e.g. residence), exposure route (e.g., inhalation), and receptor (e.g., resident.) This information will help determine priorities for setting limits. Unless evidence suggests otherwise, not all possible pathways will need to be scrutinized. For example, in its human health and risk assessment protocol for hazardous waste combustion facilities, the EPA initially recommended evaluating direct inhalation exposure pathways for all receptors. However, because available data indicated that the contributions to human health risk of combustion emissions from certain pathways (e.g., ingestion of groundwater, inhalation of resuspended dust, dermal exposure to soil) was typically small, the EPA suggested that not all pathways needed to be evaluated (EPA 1998b). A similar evaluation for refinery releases may identify pathways with insignificant contributions to human health risk that could receive lower priority. Of course, pathways with evidence indicating human health or ecological risk should be analyzed.

The above information will be used to assess exposures and to determine health-based risk levels. As the field of ecological risk assessment becomes more developed, baseline environmental information will be used to establish ecologically based risk levels as well as health-based levels. 


\subsubsection{Characterize Pollutants of Potential Concern}

Traditional environmental regulatory approaches typically limit releases of specific pollutants to the amount remaining after the best available technology has been applied to the release source. Health effects may be considered in deciding which pollutants should be regulated, but the limits themselves typically do not consider pollutant interactions, potential for harm, or other factors that reflect actual health and ecological impact. The RBB sets limits on the basis of the risks of the releases to human health and the environment. It does not require or even suggest the use of particular technologies. Rather, it allows the refinery to use whatever approaches it finds most appropriate as long as the risk-based limits are not exceeded.

Because setting risk-based limits requires significant data, analysis, and a participatory process, it will not be possible, nor necessary, for the RBB Board to establish risk-based limits for all refinery releases at the outset. Attempting to set limits for the entire array of refinery releases could overwhelm the undertaking and defeat the objective of trying to control those pollutants with the greatest contributions to overall risk. To establish limits for releases with the greatest contributions to overall total risk, information on the amount and source of releases (from the inventory) and the environment into which they enter (from the environmental characterization) must be combined with pollutant-specific data on toxicity, interactions with other pollutants, and exposure pathways. This step (pollutant characterization) applies data from a variety of sources to the site-specific refinery residuals to increase understanding of the potential harm specific residuals could contribute to public health and the environment. It thus provides information for setting priorities on residuals for which release limits will be established. Pollutants whose toxicity or exposure conditions are very uncertain could be flagged as needing additional research.

The kinds of pollutant-specific data to be collected in this step include the following:

- Potential health effects (perhaps from epidemiological studies of various subpopulations);

- Severity, persistence, and reversibility of effect;

- Toxicological data (human data would be preferable to animal data, but animal data could be extrapolated to humans if human data were not available);

- Degradation characteristics (pollutants that accumulate in the environment would generally be of greater concern than those that degrade rapidly);

- Exposure pathways (how the pollutant is transported through the environment to the human or ecological receptor);

- Exposure routes (inhalation, ingestion, dermal, etc.); and 
- Potential synergistic or antagonistic reactions with other pollutants that might be released from the refinery or other nearby sources or that might otherwise exist in the local environment.

The EPA has conducted comprehensive risk assessments that contain much of the above information for many pollutants. For some pollutants, other peer-reviewed scientific literature may contain the necessary data. For other pollutants, data may be limited, but, as noted above, risk assessment research continues to expand, so information on such pollutants will probably be available in the next several years.

Regardless of the source, the data also can be expected to reflect discrepancies and uncertainties. In addition, RBB Board members will probably disagree with each other, and data may change as new studies are conducted. These issues should not halt the process. Rather, the RBB Board should attempt to resolve such issues, and it should flag issues that cannot be resolved for further consideration when setting the limits. The objective is to use the best possible data, recognizing that changes will occur. All data should be referenced and contain notes regarding uncertainties, gaps, or discrepancies. Data not derived from scientifically peerreviewed sources should only be used in the absence of peer-reviewed data and if agreed upon by the RBB Board and scrutinized for accuracy.

\subsubsection{Screen and Prioritize Pollutants for Setting Release Limits}

Petroleum refineries can emit more than 150 individual pollutants to various environmental media (EPA 1995a). Setting priorities so that those releases with the greatest risks to the local community are addressed first will facilitate the ability to reduce or maintain total risks within acceptable levels. Several organizations have emphasized the need to prioritize pollutants for regulatory purposes. For example, in its draft summary IRP report, the SAB noted that "to make the most effective, outcomes-based decisions to improve human and environmental health," it will be necessary to "identify the priorities among and interrelationships between the major environmental and human health problems" (EPA 1999e).

Similarly, in its 1997 report, Framework for Environmental Health Risk Management, the Presidential/Congressional Commission on Risk Assessment and Risk Management recommended that regulatory agencies should "propose and implement actions that address the most significant sources of total exposure to hazards under review" (Presidential Commission 1997).

In this step, the RBB Board uses information obtained in the above steps (e.g., amounts and types of pollutants released, characterization of the receiving environment, and characterization of the pollutants) to determine priorities for establishing the risk-based limits. The exact number of high-priority pollutants will depend on refinery and local environmental conditions. Release limits would be set initially for roughly 10 to 20 pollutants.

The RBB Board needs to set criteria (e.g., pollutant toxicity, quantity released, potential exposure pathways) for ranking the pollutants. The RBB Board can develop its own criteria and weighting scheme, adapting some of the recently developed approaches for ranking pollutants 
according to risk. Four examples are summarized in the following subsections. Regardless of the method used to set priorities, the RBB Board must recognize and convey the limitations of the data, models, and methods used.

\subsubsection{California Air Toxics Program}

This integrated regulatory strategy estimates the overall risk attributable to a particular chemical rather than assessing risks from individual sources. Once the program concludes that the risk warrants action, all identified stationary, mobile, and area sources of the chemical are examined to determine the most cost-effective strategy for reducing emissions and exposure (Presidential Commission 1997). This strategy, which considers overall risk from a given air toxic, could be adapted to set priorities in the RBB. Because the strategy pertains to air toxics only, the RBB Board would need to modify it to consider cross-media and multimedia impacts.

\subsubsection{Minimal Risk Levels for Hazardous Substances}

The ATSDR developed 280 substance-specific minimal risk levels (MRLs) for hazardous constituents commonly found at toxic waste sites. These MRLs are designed to help public health professionals screen for hazardous substances that are "likely to be without appreciable risk of noncancer health effects" and thus facilitate identification of contaminants of potential concern at hazardous waste sites. These MRLs are expressed in terms of concentration for inhaled substances and in terms of daily human doses (milligrams of inhaled substances per kilogram of human body weight per day) for ingested substances (ATSDR 1999).

\subsubsection{Sector Facility Indexing Project}

The EPA's Office of Enforcement and Compliance Assistance (OECA) developed the Sector Facility Indexing Project (SFIP) to combine TRI data - which report quantities of emissions releases - with toxicity weighting factors to generate an index that reflects reported pollutant quantities and associated chronic human health hazards (EPA 1998c). Such an index is important because on the basis of TRI data alone, one could conclude that the risk associated with the emissions of 100 tons of chemical A was the same as that for 100 tons of chemical B. The SFIP data could show, for example, that because chemical $\mathrm{A}$ is a persistent bioaccumulative high-potency carcinogen, while chemical B is a short-lived, low-potency carcinogen, chemical A would produce a higher potential risk. OECA has developed the SFIP for five sectors, one of which is petroleum refining. However, the SFIP does not include exposure data. Therefore, the actual risk could be greater for chemical B than for chemical A if, for example, chemical B moved through the environment more quickly and was more readily absorbed by humans than chemical A. By not including exposure data, the SFIP may provide misleading indications about risk. Therefore, to be useful in setting priorities in the RBB, exposure data would need to be added to the SFIP data.

\subsubsection{Risk Screening Environmental Indicators Project}

Ten years ago, the EPA OPPT began the Risk Screening Environmental Indicators (RSEI) project to facilitate priority setting and to direct resources to areas with the greatest opportunities 
to reduce health and environmental risk. The idea was to integrate toxicity, exposure, and population considerations to provide a screening-level, risk-related perspective for relative comparisons of chemical releases. Thus, the RSEI project takes the SFIP a step further by adding exposure data to generate risk indicators. In July 1999, the EPA issued a report describing the RSEI model and noted strengths and limitations of the approach (EPA 1999b). The model uses the following information to derive a facility-specific, chemical-specific, and medium-specific "indicator element":

- Toxicity weight. Each chemical is scored by considering its exposure route (inhalation, ingestion) and class of effect (cancer, noncancer). Toxicity data come primarily from EPA's Integrated Risk Information System (IRIS), which contains peer-reviewed data, or from EPA's Health Effects Assessment Summary Tables (HEASTs).

- Surrogate dose. Exposure potential is estimated by using a "surrogate dose," which is developed for each medium-specific release pathway (considering physical and chemical properties and site characteristics when available). It is used to estimate the concentration of the chemical in the medium into which the chemical is released. For example, a steady-state Gaussian plume model estimates long-term pollutant concentration downwind of a stack or area source. A first-order decay equation (along with estimated river discharge and velocity) estimates surface-water concentrations for distances up to $20 \mathrm{~km}$ downstream of the release site. The model combines these concentrations with standard human exposure assumptions to estimate surrogate dose. The model adjusts the surrogate dose depending on the uncertainties associated with the estimates. (The use of assumptions and extrapolations in the absence of actual data increases uncertainty.)

- Exposed population. The model estimates the size and racial mixture of pathway-specific exposed populations by using block-level and county-level census data.

The facility/chemical/medium-specific indicator element is the product of the toxicity weight (for the specific chemical and medium), the surrogate dose (for each facility, chemical, and medium) and exposed population (for each facility and medium). The indicator elements can be used to compare chemical, facility, and medium combinations or can be added to obtain an overall indicator value. The indicators can be used to prioritize and rank for strategic planning purposes, risk-related targeting, and trend analyses.

Strengths of using RSEIs for setting priorities in the RBB include the following:

- The RSEI model includes toxicity, exposure, and population data.

- The model and data are accessible via personal computer in a Windows-based format.

- The model can perform multimedia analyses.

- The model can consider cancer and noncancer endpoints. 
- The indicator methodology is peer-reviewed (by EPA's SAB and others).

- The model contains data for 576 TRI chemicals and could be expanded to include nonTRI chemicals.

Limitations of using RSEIs in the RBB include the following:

- Some assumptions may not be realistic; for example, the model assumes that air concentrations are equal for indoor and outdoor exposures and that populations are continuously exposed.

- Not all dermal or ingestion pathways are evaluated.

- Only chronic human effects are considered; currently the model does not provide indicators for acute human health or ecological effects.

- Several technical caveats regarding toxicity, exposure, and population components of the model may limit its use (EPA 1999b). For example, exposure levels come from models rather than monitors.

The RSEI project integrates large amounts of peer-reviewed data and research to help set priorities. While the model may not be an ideal match for the RBB, it demonstrates that such approaches exist and are undergoing continual improvement. The EPA is already addressing some of the current limitations of the model, and OPPT plans to develop similar indicators for acute human health risks and chronic and acute ecological effects. The EPA states that future versions of the model may allow users to edit model data and import and export files and that a future version will be compatible with a geographical information system (GIS) format. If the RBB Board were ready to use the model and significant RSEI limitations remained, the Board could work with the EPA to address those limitations (e.g., substitute actual monitoring data for modeled data if available). It could also use local citizen input to modify assumptions as appropriate.

\subsubsection{Document and Communicate Results}

A documented baseline will provide an agreed-upon set of data readily available for use in setting risk-based limits and in changing limits as release levels, environmental conditions, and other location-specific data change and as uncertainties are added or removed. All underlying assumptions and findings of the release inventory, environmental characterization, and priority setting should be made publicly available (with the possible exception of any confidential refinery process information.) Most of this information (e.g., TRI release data) is already publicly available. Similarly, if the RSEI model were used in the screening and ranking process, the underlying assumptions and data used in the RSEI model would be publicly available; no additional effort by the RBB Board would be needed. To increase stakeholder understanding of the technical data and its significance, the RBB Board should also provide key peer-reviewed documents and Internet addresses for reference and explanatory materials. The form of documentation is not critical, but it must be straightforward, clear, and transparent. As the 
revised $\mathrm{RBB}$ develops, it is possible that a template could be prepared to facilitate baseline data collection and reporting.

All RBB participants should recognize that the baseline is subject to change. Some activities (e.g., addition of a new refinery process, significant change in crude, construction or removal of another industrial facility in the area, shifting of population toward or away from emissions sources) might affect the baseline and release limits. Similarly, public concern might increase if, for example, studies provided new information about the toxicity or transport mechanism of a given pollutant. When these types of significant changes occur, the RBB team should meet to revisit the baseline. Other, more subtle changes (e.g., short-term feedstock modifications, exchange of one similar process for another) might not (individually) significantly affect the baseline. Over time, the accumulation of these subtle, individual changes, as well as changes in the understanding and knowledge of toxicities and in exposure assumptions, might affect the baseline. Thus, a periodic (e.g., five-year) re-examination of the baseline should be undertaken.

\subsection{Set Risk-Based Release Limits in the Revised RBB}

If unlimited resources were available for environmental and human health protection, the prudent approach would be to strive for no releases. Because there are unknowns and uncertainties associated with the effects of releases on public health and the environment, the only truly safe way to ensure protection would be to mandate zero releases to the environment. However, most stakeholders agree that such an approach is not practical. The RBB aims to set release limits so that resources are targeted toward those activities and releases that provide the greatest contributions to total human and ecological risk. The goal of setting chemical-specific release limits is to ensure that refinery releases, when combined with other pollutants in the environment, will result in a total cumulative risk level that is considered acceptable by all potential stakeholders.

The following subsections address the concept of cumulative risk and how it can be used in environmental regulation and protection. The RBB addresses both public health and environmental risks. However, the methodology for setting release limits emphasizes setting limits that protect human health. The reason is because even though the amount of ecological risk information is increasing, more data and experience exist in the area of health risks. Therefore, the process is easier to depict for health risks than for ecological risks. As the scientific community increases its understanding of ecological risks, the RBB Board should use this information to set total ecological risk levels as well as health-based risk levels. The remainder of the section suggests a participatory approach for setting health-risk-based release limits so that the total cumulative risk does not exceed acceptable risk levels. Several reviewers of the RBB strawman approach cautioned that exchanging the releases of one pollutant for those of another could cause the releases of one or more particular pollutants to increase significantly, thereby increasing human or ecological impact. As a result, the revised RBB, while allowing for the exchange of releases across media and pollutants, also establishes certain pollutant-specific limits that cannot be exceeded. (Standard risk assessment methodologies would be used to ascertain these pollutant-specific limits.) 


\subsubsection{Cumulative Risk}

The term cumulative risk connotes different things to different observers. For the RBB, the term cumulative risk refers to the potential risks presented by multiple stressors in the aggregate, and it captures the fact that combinations of pollutants from various sources through various environmental media over various time periods affect human and ecological receptors.

The importance of cumulative risk in protecting the nation's public health and the environment was highlighted in a July 1997 memo from EPA Administrator Carol Browner and Deputy Administrator Fred Hansen to all EPA offices (Browner and Hansen 1997). The memo asked all offices to ensure that all major risk assessments embrace cumulative risk assessment "so that we can better advise all citizens about the environmental and public health risk they face, and improve our ability to protect the environment and public health for the nation." It said that "today, better methods and data often allow us to describe and quantify risks that Americans face from many sources of pollution, rather than by one pollutant at a time. We are increasingly able to assess not simply whether a population is at risk, but how the risk presents itself." Accompanying the memo was guidance developed by EPA's Science Policy Council that directs each office to integrate multiple sources, effects, pathways, stressors, and populations for cumulative risk analyses. The guidance acknowledges that current statutory constraints, combined with limited data and relatively limited experience in the practice of cumulative risk assessment, challenge researchers' abilities to address cumulative risk. However, it also says that the EPA will support research to improve the understanding of cumulative risk and to develop methods that account for the multiple elements of risks that affect humans, animals, plants, and their environment.

A number of ongoing cumulative risk-related projects serve as models for setting release limits in the RBB. While not directly transferable, they each provide applicable data or methodologies. Five such efforts are summarized below.

1. Chicago Cumulative Risk Initiative. This initiative involves several efforts to measure and reduce cumulative risks to Chicago metropolitan area residents. Responding to a citizen's suit that questioned approving industrial air permits on a site-specific rather than a cumulative basis, the project provides for collecting information and planning actions to address multimedia pollution sources. The project includes the following components:

- Generation of an environmental loading profile to establish quantities and destinations of toxics released into the Chicago-area environment (similar to the baseline inventory in the RBB);

- Facilitated workshop to discuss loading profile data, risk assessment, and pollution prevention/remediation options (similar to the public participation aspects of the RBB); 
- Development of a scientifically valid methodology for approximating the cumulative hazards for local residents (a step necessary for setting the risk-based releases in the $\mathrm{RBB}$ ); and

- Implementation of pollution prevention/remediation activities (akin to the compliance assurance component of the RBB).

The screening phase of the cumulative risk assessment, which focuses on air toxics and their sources in a two-county area, is underway. Goals of the screening phase include developing a basis for selecting study sites, mapping the "hazardscape" in the Chicago area, assessing cumulative hazards, and gathering information on susceptible populations (Butler and Nieves 1998).

2. National Human Exposure Assessment Survey (NHEXAS). This interagency program is designed to address some of the limitations of traditional single-chemical, singleexposure route approaches to environmental regulation (EPA 1999d). The NHEXAS team randomly selected and asked hundreds of subjects in three separate regions to participate in a study that (1) measures levels of chemicals in the air they breathe; the food, water, and beverages they consume; and the soil and dust around their homes; (2) measures chemicals in biological samples (e.g., blood, urine); and (3) identifies via questionnaires possible sources of exposure to chemicals. The study seeks to:

- Identify subgroups that may be highly exposed or susceptible to chemicals in their environment,

- Relate known pollution sources to actual exposures in people,

- Provide a baseline of normal exposure ranges of chemicals to compare with results from other investigations conducted at particular sites of concern or from specific routes,

- Evaluate and improve the accuracy of models developed to predict exposures of people to chemicals, and

- $\quad$ Test different techniques for conducting multimedia human exposure studies.

Chemicals include volatile organic compounds, including benzene and trichloroethylene, and metals and pesticides. Sample collection began in mid-1995, and peer-reviewed results are expected to be available in 2000 .

3. Total Risk Integrated Methodology (TRIM). The EPA OAQPS is designing a multipollutant, multimedia, multipathway assessment model to help evaluate and regulate health risks from air emissions. The model, which is designed to be flexible and used by a variety of stakeholders, will characterize uncertainty and variability. When complete, it will contain several modules, including the following: 
- An environmental fate, transport, and exposure model to predict movement of the pollutant mass through the ecosystem;

- A pollutant uptake model to determine the quantity of pollutant that entered an organism during a specific exposure event;

- A biokenetics model to estimate health effects resulting from pollutant uptake; and

- A risk characterization model to present risk estimates, assumptions, and uncertainties.

In its 1998 review of TRIM, EPA's SAB found the model to have a technically defensible approach and a flexible structure to accommodate changes and integration with other data sources (e.g., GIS). It also found that the model is consistent with EPA's ecological and human health risk assessment guidelines. Areas of concern include the massive amount of effort needed to meet input data needs, the assumptions about uniform exposure distributions, and the limited use for criteria air pollutants. SAB recommended testing the model with real data and working with potential users to refine it (EPA 1998a).

4. Guidance for Conducting Health Risk Assessments of Chemical Mixtures. In 1986, the EPA issued a 20-page guide describing broad concepts related to toxicities and exposures associated with chemical mixtures (EPA 1986). Since then, the EPA has worked continuously to refine this guidance. In May 1999, it issued a 150-page supplement that reflects "the evolutionary scientific development in the area of chemical mixtures risk assessment" (EPA 1999h). The guidance defines mixtures as any combination of two or more chemical substances, regardless of the source or of the spatial or temporal proximity that can influence the risk of chemical toxicity in the target population. The 1999 guidance is designed to assist decision makers by characterizing health risks for particular exposure conditions. Emphasizing that the quality and quantity of pertinent information available for risk assessment varies considerably for different mixtures, the guidance describes procedures that can be used, depending on the nature and type of available data (EPA 1999m). The guidance includes procedures for three types of data: data on the mixture of concern, data on a toxicologically related mixture, and data on the component chemicals of the mixture. On May 20-21, 1999, an outside scientific panel reviewed the draft guidance. While it expressed concern over several areas (e.g., the guidance concentrated on hazard identification and dose-response assessment and did not address exposure assessment), the panel chairman said that the "guidance will be a good interim step toward a future ideal world of comprehensive, aggregate, cumulative chemical risk assessment and public health risk management" (BNA 1999a).

5. Human Health Risk Assessment Protocol for Hazardous Waste Combustion Facilities. The 1996 preamble to the proposed MACT rule recommended that site-specific risk assessments be conducted as part of the RCRA permitting process for hazardous waste combustors. Finding that in many cases, risks from indirect exposure (pathways other 
than direct inhalation) can constitute the majority of risk from a hazardous waste combustor, the EPA prepared a protocol in 1998 to help assess risks of hazardous waste combustors from direct as well as indirect pathways (EPA 1998b). The protocol provides procedures for assessing the risks, explains the basis for the procedures, and includes data sources needed to complete the assessments. The protocol calls for the use of existing, site-specific information in the risk assessment, and it recommends a process for evaluating reasonable (not theoretical, worst-case maximum) potential risks to receptors. It provides guidance on compiling facility baseline information, including identifying compounds of potential concern, modeling transport and fate, identifying exposure scenarios, estimating media-specific contamination, estimating exposure, characterizing cancer and noncancer risks, and documenting uncertainties.

No single existing model can project the total cumulative risk associated with releases of all refinery pollutants combined with all other pollutants to which human and ecological populations may be exposed from all pathways and all media. However, the above examples illustrate that the science of risk assessment is moving in the direction of developing tools to conduct such assessments. Several factors are driving such development. These include legislative mandates (e.g., the Food Quality Protection Act of 1996, which requires estimating cumulative risk of exposure to pesticides in the diets of children); citizen requests for new types of risk analyses (e.g., identifying the risks of siting hazardous waste facilities in the context of existing health risks); and several reports from various advisory panels urging the EPA to design regulations that reflect mixtures as found in the environment rather than single chemical risks. Thus, the development of the RBB is consistent with current trends that recognize the importance of cumulative risk and with the data collection and modeling efforts required to estimate such risk.

\subsubsection{Process for Setting Risk-Based Limits}

The process for setting risk-based limits depends on several factors, including, but not limited to, site-specific conditions, stakeholder input, and changing knowledge. The following paragraphs suggest an approach that expands on the initial strawman approach and attempts to address comments made during the workshops. Because this is a future-oriented approach, some of the details will be determined as the science of risk assessment grows. All decisions required in each of the following six steps would be made by the RBB Board, whose members would include representatives of the refinery, the relevant regulatory bodies, and local citizens.

1. Determine acceptable risk.

2. Calculate an initial total cumulative risk estimate on the basis of existing baseline emissions and emissions from surrounding sources.

3. For priority pollutants identified in the baseline, establish initial release targets.

4. Develop alternative limits for individual pollutants, providing for exchanges across pollutants and media, as long as total risk is not exceeded.

5. Document agreed-upon release limits and make them accessible to the public. 
6. Conduct periodic reexaminations of release limits to incorporate new information.

\subsubsection{Determine Acceptable Risk}

The overall objective of the RBB is to ensure that refinery releases, when combined with releases from other sources, do not adversely affect human or ecological health. Given the inability to translate pollutant releases into actual health effects, the generally agreed-upon surrogate measure is risk. Thus, it is important to note that cancer risk is an incremental probability that an individual will develop cancer over a lifetime as a result of exposure to a potential carcinogen; it is not a conclusion that the individual will necessarily die as a result of exposure to the potential carcinogen. Scientists and nonscientists have written volumes on the uses and misuses of risk and risk assessment, and the project team acknowledges that the term "risk" can trigger an automatic reluctance to move toward further discussions. One recent report (EPA 1999e) suggests an alternative terminology, ${ }^{6}$ and the revised RBB could be recast to use this terminology. However, the term "risk" will continue to be used in this description because it serves as a useful way to express the general desire to protect human health and the environment in a scientifically defensible manner. Similarly, it is important to note that many of our existing regulations that seek to protect human health contain implicit or explicit risk goals.

The 1989 National Emission Standards for Hazardous Air Pollutants (NESHAP; 54 FR 38044) for benzene illustrates how the EPA employs the concept of "acceptable risk" to protect public health (EPA 1989). In the preamble to that standard, the EPA explained that in protecting public health with an ample margin of safety under Section 112 of the CAAA, it strives to provide "maximum feasible protection" against health risks from hazardous air pollutants by (1) protecting the greatest number of persons possible to an individual lifetime risk of no greater than roughly one in one million and (2) limiting to no higher than roughly one in ten thousand the estimated risk that a person living near a plant would have if he or she were exposed to the maximum pollutant concentrations for 70 years. These goals are implemented via a two-step process, which stems from a 1987 court decision (NRDC v. EPA, 824 F.2d at 1146, 1987) known as the vinyl chloride decision. In that decision, the court required the EPA to (1) determine a "safe" or "acceptable" level of risk based on a judgment of "what risks are acceptable in the world in which we live" considering only health factors and (2) set a standard that provides an "ample margin of safety" that may consider "costs, feasibility, and other relevant factors."

In the first step, the EPA considers the estimated risk to an individual exposed to the maximum level of a pollutant for a lifetime (the maximum individual risk or MIR). The EPA generally assumes that the risk to that individual is acceptable if it is less than or equal to one in

6 Rather than expressing cancer risk in terms of predicted incidence or numbers of deaths per unit population, the "margin of exposure" expresses risk as the ratio of a specified dose derived from a tumor bioassay, epidemiologic, or other study (such as the dose associated with a $10 \%$ response rate) to an actual or projected human exposure. A lower margin of exposure indicates a greater concern. The indicator is similar to the margin of protection indicator used in "hazard quotient" (HQ) approach for noncancer risk assessment. The HQ compares an estimated exposure to a reference dose (Rfd) or reference concentration (Rfc) value (EPA 1999c). 
ten thousand. The MIR is developed on the basis of conservative assumptions, such as continuous exposure for 24 hours per day for 70 years. The EPA recognizes that such a conservative risk level does not necessarily reflect true risk but rather an upper bound that is unlikely to be exceeded. Thus, the EPA considers an MIR of roughly one in ten thousand to be the upper end of the range of acceptability. As risks increase above this level, they become less acceptable and would be weighted in conjunction with other health risk measures and information in making an overall judgment on acceptability. These factors include, but are not limited to, the following: the overall incidence of cancer or other serious health effects within the exposed population, estimation uncertainties associated with the risk measures, weight of scientific evidence for human health effects, and effects that result from the co-location of facilities.

In developing this limit, the EPA first conducted a Survey of Societal Risk to place risk estimates in perspective and to provide background and context for its judgment on the acceptability of risks in the world in which we live. In the survey, the lifetime risk of premature death ranged from one in ten to one in ten million, and incidence levels ranged from less than one case per year to as high as 20,000 cases per year. On the basis of this survey, the EPA concluded that no specific factor in isolation could be identified as defining acceptability under all circumstances and that acceptable risk depends on a variety of factors and conditions. Nonetheless, the EPA concluded that the presumptive level of approximately one in ten thousand established for the MIR is within the range for individual risk in the survey and provides health protection at a level lower than many other risks common in the world in which we live.

Second, the EPA set the actual standard at a level that provides "an ample margin of safety," considering all health information, including the number of persons at risk levels higher than approximately one in one million and other relevant factors such as cost, economic impact, and technological feasibility.

The determination of acceptable risk provides a health- and ecology-based target. The RBB Board also could use two acceptable risk levels: one for the general population and one for susceptible populations. For example, it could begin with the acceptable risk level for the general population and then adjust it (e.g., make it more stringent) on the basis of the number of people near the refinery who are subject to higher risks. The important element is that the Board must fully evaluate the initial acceptable risk target. In so doing, it might seek input from interested outside parties. Once the acceptable risk level is agreed upon, the Board should document it as such. Some workshop participants raised philosophical questions as to who determines acceptable risk, and some suggested that the determination should be made at the national level. If the RBB is adopted broadly, such national-level decisions may be appropriate. However, initially, determinations should be made by the local, site-specific RBB Board.

\subsubsection{Calculate Initial Estimate of Total Cumulative Risk}

By using whatever models constitute the state of the art in cumulative risk assessment at the time of implementation, the RBB Board would develop an initial estimate of total cumulative risk to affected populations from all sources, including refinery releases. The models would use data from the refinery's inventory of releases and similar inventories from other sources, 
information on exposure pathways and environmental conditions developed during the environmental characterization, and information developed on the toxicities, exposure potentials, and, where possible, monitored (rather than modeled) pollutant concentrations developed in the baseline. Affected populations would include the following: (1) the residential population exposed to releases from the refinery; (2) susceptible populations (e.g., elderly, children) exposed to refinery releases; and (3) populations exposed to refinery releases that are transported long distances. Contributions to this total risk would be identified by chemical, medium, and source, facilitating the identification of the refinery's contributions to total risk.

As more information becomes available on pollutant toxicities, interactions among pollutants, cause-and-effect relationships, fate and transport, dose-response relationships, and other subjects needed to calculate total cumulative risk, the development of a modeling system that will use this information in conjunction with site-specific data to calculate total cumulative risk and the risks associated with individual pollutants is anticipated. Such a system will allow users to estimate not only cumulative risk but also to identify the contributions of individual pollutant risks to total cumulative risk. Ultimately, such a system will also include ecological risks as well as health risks, and it will account for seasonal variations (e.g., ozone and nonozone seasons). Because many of the remaining steps in the RBB methodology to set limits and assure compliance will use such a modeling system, the eventual existence of a cumulative risk estimating and assessment model (CREAM, the modeling system described above) is assumed.

\subsubsection{Establish Initial Pollutant-Specific Risk-Based Release Limits}

If the initial total cumulative risk estimates calculated in CREAM were less than the acceptable risk(s), the RBB Board would still develop pollutant-specific release limits. Pollutantspecific release limits can help ensure that releases of specific pollutants do not result in excess risk or "toxic hot spots." As noted above, some workshop participants cautioned that unlimited exchanges of pollutant releases could, theoretically, lead to situations in which releases of a pollutant with very high control costs would be traded by all sources, so even if other pollutant releases were reduced and the overall risk was still acceptable, the effects from the increased releases of the costly pollutant could cause public health or ecological damage. Ultimately, CREAM would identify such increases before such trades occurred, but in the interim, pollutantspecific limits would be established to prevent such increases. Pollutant-specific release limits also provide a refinery with the option to use different (more cost-effective) methods to meet compliance objectives, and they provide targets against which refiners can compare alternative control strategies for different pollutants. The compliance assurance component of the RBB provides additional incentives for the refinery to reduce releases beyond those to meet acceptable risks, thus providing for continuous improvement. While incentives can apply to the total risk reduction, they can also be directed toward specific pollutants that may have greater risks than others.

Two types of release limits would be established for pollutants on the baseline priorities list. The first type would be the maximum risk-based release limit, which could not be exceeded, even with trading. The second release limit would apply if the releases of that pollutant were to be exchanged with those of another pollutant while maintaining a total cumulative risk less than the total acceptable risk limit. 
To set the maximum risk-based release limits that could not be exceeded (e.g., by exchange or trading), standard, site-specific, single-pollutant risk assessment methods would be used. These methods would use the data collected in the baseline (release inventory, environmental characterization, pollutant characterization), combined with an acceptable single-pollutant risk level, to "back-calculate" release limits that could not be exceeded. These limits would be calculated by using site-specific data and would be developed for each pollutant in the priority list and for each environmental medium through which human or ecological receptors would be exposed.

For pollutants subject to exchange, CREAM would identify relative contributions of different pollutant releases to total cumulative risk. For each pollutant, the initial target would be the release limit that resulted in a total cumulative risk that was less than or equal to the total acceptable risk, assuming releases of all other pollutants remained constant at the baseline inventory level. Because the idea would be to increase flexibility for the refinery while assuring human and ecological protection as measured by acceptable risk, these targets could be modified as other pollutant releases would increase or decrease, as long as the overall acceptable risk was not exceeded. In the early implementation stages of the RBB, significant uncertainties and variabilities will exist, because information is missing on many issues that are poorly understood, complex, or difficult to quantify accurately. For example, data uncertainties can result from equipment limitations and sampling errors, and modeling uncertainties can result from a variety of input assumptions (e.g., dose-response models, animal-to-human data extrapolations.)

Some stakeholders may argue that these uncertainties are too great to allow for the development of reliable limits. However, few, if any, environmental regulations have ever been developed on the basis of definitive, undisputed data and models; therefore, some uncertainty is acceptable. Nonetheless, if stakeholders believe that CREAM is underestimating risk, the RBB Board can tighten the limits to reflect such uncertainty. Such adjustments would help assure concerned parties that modeling and data uncertainties will not lead to unprotective limits. They would also provide incentives to reduce uncertainties by collecting more data or conducting more scientific research. For example, the project team expects that as measurement technologies continue to improve, the contributions to overall total risk from the various pollutants will reflect real-time measurements rather than modeled contributions. Specifically, uncertainty could be captured in the limits according to a table that would list, for each pollutant, the key sources of uncertainty (e.g., assumptions, model algorithms, accuracy of input data), the amounts of such uncertainty (high, medium, low), how these uncertainties could affect the limits (overestimate, underestimate, no significant effect), the magnitude of effect (e.g., factor of 2), and the resulting, adjusted limits. The RBB Board would ensure that all limits were documented and that the underlying data and assumptions would be publicly available.

\subsubsection{Develop Alternative Risk-Based Release Limits}

Alternative risk-based release limits (i.e., alternatives to existing release limits) give the refiner the flexibility to use new technologies, incorporate pollution prevention and process changes, and take advantage of other options controlled by the refinery to protect human health and the environment. With the exception of not exceeding the maximum single-pollutant riskbase release limits established in the previous section, the refinery will be able to exchange 
releases among pollutants as long as total cumulative risk does not increase beyond the acceptable risk level. Before actually implementing CREAM, the RBB Board will use CREAM to develop alternative risk-based limits for the various pollutants. With such testing, the refinery can plan cost-effective approaches to reduce risk and can obtain feedback from the RBB Board and other interested parties on potential impacts. Once the alternative release limits are developed, they will be documented and used in CREAM. CREAM will run constantly, so that the cumulative risks will be estimated, and any variations will be recorded. This process will provide the RBB Board (and others) with real-time data regarding the compliance status of the refinery. If the risk level begins to approach the acceptable level, the refinery will take immediate steps to ensure that the risk is not exceeded. Similarly, if the refiner wants to make minor changes in pollutant releases, the model can be used to test the changes, and if the total risk remains constant or declines, such changes could be implemented. Major changes may require RBB Board review.

Advances in computer technology and data presentation will facilitate the implementation of this step. For example, CREAM, which governs the generation of release limits based on total risk, is expected to contain GIS capabilities enabling the ready identification of potential "hot spots." Such identification will not only allow the Board to develop means to address such potential hot spots by adjusting refinery releases, but it may also be used to flag other, potentially more cost-effective mitigating measures.

\subsubsection{Document and Communicate Results}

The maximum risk-based release limits will be documented and could be made available to the public via avenues such as the Internet. Besides posting the risk-based release limits, the RBB Board could also place CREAM on the Internet, enabling interested parties to view (but not change) the underlying data and assumptions. The public could also comment on the model during the initial implementation. Through such public comment, potential gaps might be identified, errors might be uncovered, and additional data sources might be identified. These review comments are expected to lead to model improvements that will ensure model credibility before actual use. Internet posting could allow interested parties to comment on an ongoing basis and the Board to integrate new information as appropriate. In addition, monitors would collect data electronically, so the public would be able to review not only the model results but also the model inputs on a real-time basis. This pubic availability responds to comments made by workshop participants who indicated a need for more public access to data to increase trust.

\subsubsection{Periodically Reexamine Release Limits}

As noted, information on pollutant interactions, health and ecological impacts, and numerous other factors that pertain to risk will increase over the next several years. To ensure that such information is incorporated into the process, the RBB Board will schedule periodic meetings to provide opportunities for stakeholders to present and review such information. This information may or may not lead to the modification of release limits. For example, if new data

indicate that a particular chemical has a previously unknown but significant effect on a sensitive population, the risk-based limits need to reflect this information. If the refiner is made aware of this issue, it could investigate alternative means to reduce its releases of that chemical. The 
refiner could also support research on fate and transport mechanisms, and, depending on the results, suggest alterative approaches for the sensitive populations to reduce the risk. Thus, new information does not necessarily trigger a release limit change.

The process for changing limits on the basis of new information will help ensure against arbitrary changes. RBB Board members will have reasonable time frames to acquire the data needed to support or refute the new information. Changing the limits will require the same deliberative process used to set the original, or previously established, risk-based release limits.

\subsection{Assure Compliance in the Revised RBB}

The RBB assures compliance through the use of direct, real-time monitoring of releases and resulting concentrations that are linked to the cumulative risk model (CREAM). Acknowledging the need for refinery flexibility, workshop participants emphasized that increased flexibility calls for increased accountability. The RBB provides maximum flexibility to the refinery by allowing it to develop and use innovative technologies, redesign processes, change catalysts, implement pollution prevention, and so on to meet the risk-based limits. The revised RBB also requires that the results of these actions be made available, in a clear and transparent fashion, to all stakeholders. As described in Section 5.5, CREAM estimates total cumulative risk from all refinery releases to all media in the context of local environmental conditions, and CREAM includes releases from other nearby sources. The model accounts for variations in refinery releases of specific pollutants in real time. These model results could be posted electronically on the Internet, along with explanatory information on chemicals, the model, and other contextual information to facilitate data interpretation.

Such real-time data dissemination would benefit all stakeholders. First, it would respond to the commonly expressed concern that citizens groups do not have access to refinery or regulatory data. Instant access could be available electronically over the Internet, and those without Internet access (expected to be few in the context of the time frame for this approach) could obtain hard copies of data from the RBB Board, the refinery's public affairs office, or the regulatory body. Any apparent anomalies could be brought to the attention of the RBB Board via the Internet or other communication methods and be addressed in a timely fashion. Second, real-time data dissemination would serve as compliance documentation, thereby reducing the reporting burden not only for the refinery but also for the regulatory body, which currently must collect, review, and file the reports required by existing environmental regulations.

Because its goal is to attain and maintain acceptable risk levels, the RBB has no sourcespecific or pollutant-specific technological requirements. Instead, a refiner can evaluate and select the most efficient and cost-effective means of pollution control, on the basis of its particular location, capital plant, and operating conditions. The refinery will be in compliance if it meets the following three requirements:

1. Refinery releases do not result in a total cumulative risk that exceeds the agreed-upon acceptable limits.

2. Pollutant-specific risk-based maximum levels are not exceeded. 
3. Data on actual releases and resulting risk levels are publicly available via the Internet.

The remainder of this section provides additional details on the following specific compliance assurance topics:

- Measures to be developed and overseen by the RBB to assure compliance;

- The importance of using data collected from monitors as opposed to data developed through models;

- Risk-based exchange issues;

- Performance evaluation; and

- RBB implementation issues.

\subsubsection{Compliance Assurance Measures}

The RBB Board would most likely develop the compliance assurance measures as it develops the risk-based limits, since both the development and enforcement of those limits use CREAM. The Board would meet regularly to review compliance issues (e.g., reporting) and other topics requiring such periodic review (e.g., pollutant-specific limits, baseline assumptions). When necessary, the Board could schedule interim meetings to address issues that would need attention before the next regularly scheduled meeting.

This section discusses compliance assurance measures that the RBB Board needs to develop, agree upon, and implement. These measures include the following:

\subsubsection{Permits}

The RBB does not use pollutant- or source-specific permits. Instead, the RBB Board develops an overall agreement that describes the baseline inventory and conditions, how initial pollutant-specific targets are set, and how they will be allowed to vary as long as total acceptable risk level is not exceeded. The agreement describes the modeling system (e.g., CREAM) that is used to develop the release limits and to calculate resulting risk estimates needed to evaluate and ensure compliance. The agreement also defines the penalty structure for violations and reporting requirements, and it provides incentives to collect and use monitored (as opposed to modeled) data. Relevant regulatory and refinery personnel and all RBB Board members sign the agreement.

\subsubsection{Incentives and Disincentives}

The RBB is an incentive-based system, and the primary incentive is the increased flexibility to implement nonprescriptive, cost-effective, and efficient approaches. The required public reporting and accountability provides a strong compliance incentive. However, to ensure against 
lax behavior or system abuse, monetary and/or other penalties will be assessed for the following actions:

- Exceeding the risk-based release limits;

- Failing to maintain the proper functioning of monitors (e.g., assuring accuracy, minimal downtime); and

- Failing to maintain the reporting system or generating inaccurate reports.

The RBB Board determines the penalty structure. Nonmonetary disincentives could include tightening the risk-based release limits or requiring the installation of better monitors. Both the monetary and nonmonetary disincentives could be structured to increase as the degree of offense would increase or as the number of repeated occurrences would increase. The RBB Board should ensure that the penalties are large enough to discourage noncompliance. Additional disincentives may be developed through the use of punitive penalties such as those provided in the Acid Rain Program. For example, for each ton of $\mathrm{SO}_{2}$ emitted above the allowed amount, the Acid Rain Program imposes an automatic penalty of $\$ 2,000$ per ton (indexed yearly for inflation) and a reduction in allowance holdings. A recent U.S. General Accounting Office (GAO) study reported that this monetary penalty is about 25 times higher than current allowance prices (GAO 1997a). The RBB board may want to work with taxation authorities to ensure that the refinery cannot deduct the penalties as operating or other costs.

\subsubsection{Reports}

Public reporting of clear, well-defined, and easily understood compliance measures is a cornerstone of the RBB, and publicly available information is expected to provide a powerful compliance incentive. The RBB Board could use an Internet-based reporting system, which would include a variety of reports. Users could select reports from a menu that would list the names and provide one-line descriptions of each report. The RBB Board would determine the form and content of the reports. Suggested reports include the following:

- Pollution prevention and other refinery actions. This report would highlight actions taken by the refinery to reduce pollutant releases and to engage in community outreach activities. Serving to showcase positive actions, it could highlight specific technologies, processes, and other approaches. Web-based reports issued by certain manufacturing establishments and coordinated by the Chemical Manufacturers Association could serve as a model for this report. ${ }^{7}$

- Penalties. This report would provide information on penalties assessed on the refinery. It would include the reasons for, dates of, and nature of penalties (e.g., monetary, release limit adjustment).

7 The Chemical Manufacturers Association-sponsored Internet site provides data on (among other things) environmental, health, and safety performance and community service activities for more than 100 U.S. manufacturing sites. The address is http://www.chemicalguide.com. 
Summary of release limits and resulting risks. This report would contain real-time reporting (generated by CREAM) of the estimated total cumulative risk to the community and the contributions of the refinery to that risk. To facilitate interpretation, the report would also include a summary, in laymen's terms, of the purpose and methodology of CREAM, and other explanatory information to put the risk results in context. The report would also provide links to other sources (e.g., the Agency for Toxic Substances and Disease Registry) that provide information on toxicity, pathways, exposures, and other characteristics of individual pollutants.

- Data used to develop the risk estimates. This report would show the data used in CREAM and identify the data sources (e.g., toxicity data from EPA sources). The report would identify any models used to generate data and the underlying assumptions. It would identify sources of uncertainty and explain the processes used to reflect such uncertainty in the release limits.

- Monitoring information. This report would provide details on the monitors, such as the types of monitors used, their locations, reliability, sources of uncertainty, and downtimes.

- Public participation. This report would highlight how public participation is integrated into the RBB decision-making process. It would identify, for example, new information produced by the community and how such information has been or could be used to adjust risk-based limits.

Once CREAM is operating and the report formats are designed, the reporting will require little human intervention. Labor will decrease, and accuracy and consistency will increase. For example, CREAM will generate the summary risk reports and the reports that provide data assumptions and inputs used to develop the risk estimates. Similarly, the monitoring information reports will come in large part from the monitors themselves or from the overall monitoring support system. The remaining reports (the refinery pollution prevention report, the penalties report, and the public participation reports) can be updated as necessary. Reports and monitoring results should be kept for a minimum number of years (e.g., five) in case they are needed to check for errors or resolve disagreements.

\subsubsection{Site Inspections}

Because compliance is assured by the refinery's application of cost-effective and efficient methods, and no specific technologies are required, the RBB envisions relatively few inspections. Should the refinery exceed any of the risk-based limits, the state or local regulatory agency may come on the site to help identify and remedy the problem. Routine site inspections in the RBB are generally limited to inspections of the exposure-point monitors to ensure that they are working properly. The reduced need for site inspections is expected to save refiner and regulatory resources. 


\subsubsection{Periodic Reviews}

Because processes, scientific knowledge, and environmental conditions change, the RBB Board should require periodic reviews of the approaches for setting limits and exchanging releases. New information can support increasing or decreasing release limits to ensure that the overall program goals are being met. Required reviews could occur every five years, and the refinery, regulator, or local community could petition for interim reviews if sufficient information were to warrant them.

\subsubsection{Preference for Monitored Exposure-Point Data}

Actual data derived from accurate monitors offer numerous benefits over modeled data. Few stakeholders trust models. Models embody assumptions regarding input data and algorithms and can lack credibility and consistency. Because models require assumptions about complex transport and fate mechanisms and interactions with other pollutants that are not transparent to the user, real data collected via monitors at the receptor's exposure point will provide a better indication of potential health or ecological risks than will modeled data.

The emphasis of traditional regulatory programs on end-of-pipe treatments has led to the design and development of monitors that measure pollutants at the release point (e.g., a stack) rather than at the exposure point (e.g., a human). Because regulatory agencies often specify monitoring/measurement equipment designs, and because there is no demand (under the current regulatory system) for monitors that measure exposure-point concentrations, manufacturers spend few research and development dollars to develop these types of monitors. Although monitoring systems that identify and measure pollutant concentrations along the perimeter (i.e., fence line) of the refinery are commercially available, and monitors to measure concentrations at other exposure points are being developed, such systems are expensive.

The recent installation of fence-line monitors at the Tosco refinery in Rodeo, California, illustrates how new technologies can be implemented when there is some outside impetus (Oil and Gas Journal 1999). In 1994, the Tosco refinery, part of which is adjacent to a residential community, released a large amount of catacarb, a carbon dioxide $\left(\mathrm{CO}_{2}\right)$ absorber used to purify hydrogen. Following the release, local community groups pressured the state regulatory agency to deny permits for new construction until certain conditions were met. One of these conditions was the implementation of a fence-line monitoring system - something that had never before been required of a refinery. To identify the most appropriate technology for the system, Tosco

established a "fence-line working group" consisting of community and refinery representatives to review information on available technologies from various manufacturers. The working group ultimately selected a system that used three different technologies to identify, monitor, and measure 38 chemicals released by the refinery. The system displays real-time data from the monitoring systems on a computer screen and produces an alarm if certain levels are exceeded. The fence-line working group, the refinery, and the local health department agreed to these levels. The refinery maintains the monitoring infrastructure, but to avoid potential credibility concerns, a third-party company collects and supplies data summaries to the community and local authorities. 
Implementation of the fence-line monitoring system at the Tosco refinery highlights several lessons relevant to the revised $\mathrm{RBB}$. These include the following:

- Local citizen participation in decision making is increasing, and it is possible that this particular case could set a precedent for other citizen-initiated suits. Implementation of the revised $\mathrm{RBB}$, with its ongoing public participation provisions, could help limit the number or severity of such suits.

- The affected public increasingly provides the impetus to change the environmental regulatory system. As a result, including public representation in the decision-making process can be more efficient and less costly in the long term than litigation.

- Many of the technologies required by the RBB (e.g., fence-line monitors) are being developed or can be developed, given a strong-enough incentive.

- Incentives such as court cases or regulatory changes can drive technology development.

- Working groups consisting of refinery, community, and local government agencies successfully investigated and selected a state-of-the-art fence-line monitoring system and jointly determined maximum release levels. The RBB relies on such joint research, development, and decision making to reach mutually agreeable and environmentally beneficial solutions.

Without a court case or similar directive, it is unlikely that refineries that have already installed source or stack monitors would install additional monitors. However, the RBB does not necessarily require stack monitors, so over time, as technology develops and increased demand helps lower their cost, exposure-point monitors may substitute for release-point monitors. Also, depending on the size and nature of the refinery, fewer monitors might be required to characterize pollutant concentrations at exposure points than at the stack. Other international and national activities may spur the demand for exposure-point monitors. For example, fence-line monitoring systems are being developed and marketed in Europe and Japan. In the United States, the EPA recently issued a concept paper for a national air toxics monitoring program, in which it says that as new assessment tools are developed to address risks associated with toxic emissions, the EPA will modify its current goal of reducing emissions to focus on risk reductions associated with exposure to air toxics. In working toward this risk-based goal, the EPA says it will focus on the cumulative effects of air toxics, multimedia effects on water bodies, and effects on sensitive populations. "We have a long-term goal of eliminating unacceptable risks of cancer and other significant health problems from exposures to air toxics and to substantially reduce or eliminate adverse effects on our natural environment" (EPA 1999f). To meet these goals, the EPA plans (among other activities) national, regional, and community-based initiatives to focus on multimedia and cumulative risks; expanded air toxics monitoring; and national- and local-scale air quality and exposure modeling. 


\subsubsection{Accounting for Uncertainty in Risk-Based Compliance Estimates}

As noted above, the cumulative risk estimating and analysis model (CREAM) calculates total cumulative risk estimates and provides for adjustments in refinery releases to ensure that total risk is less than or equal to acceptable risk levels. When actual data are unavailable, CREAM uses modeled data. For example, when data cannot be obtained from exposure-point monitors, data are obtained from stack monitors and used in complex transport and fate models to estimate exposure-point concentrations; these estimates are used to estimate risks. However, fate and transport model assumptions can contribute significant uncertainty to the results. The revised RBB seeks to reduce the need for modeled data in CREAM (thereby increasing the reliability and certainty of the risk estimates) to the greatest extent possible. As such, it provides incentives to substitute real (monitored) data for modeled data. Risk estimates calculated on the basis of modeled data reflect the uncertainty caused by the use of modeling assumptions via an upward adjustment to the calculated cumulative risk estimate. In other words, all else being equal, a cumulative risk estimate derived by using modeled data will be adjusted upward (i.e., refinery releases result in a higher total cumulative risk) when compared with a cumulative risk estimate derived on the basis of actual, monitored data at the receptor point. Therefore, more control would be required to keep risk levels within the acceptable range.

\subsubsection{Penalty Structure}

A similar incentive (or disincentive) for using monitored data occurs if release limits are not met (i.e., if the total cumulative risk estimate derived from CREAM is higher than the total acceptable risk level, or if a pollutant-specific risk estimate calculated by using CREAM is higher than the maximum risk-based release limit.) If modeled data were used to develop the risk estimate, the penalty would be greater than if monitored data were used. ${ }^{8}$

\subsubsection{Performance-Based Measurement Systems}

Although the CREAM results ("the bottom line") are presented in terms of risk, the underlying measurements and calculations must be available for public and regulatory review. Under the current regulatory system, facilities typically prove that they are meeting specified emissions limits by using measurement technologies approved, endorsed, or prescribed by the EPA. However, adherence to such prescribed technologies provides no incentives to, for example, develop options to reduce laboratory costs or develop innovative, more cost-effective measurement technologies; it can, in fact, result in inferior data measurements. The revised RBB would build on an existing EPA initiative, known as performance-based measurement system (PBMS) procedures, under which facilities can use alternative measurement systems (BNA 1999b). Such alternative systems would reflect the specific needs of the individual refinery. The refinery could thus demonstrate compliance by using any scientifically appropriate analytical test method, provided the method meets EPA-established performance criteria for characteristics such as precision and accuracy. Thus, flexibility is allowed in the way that measurements are

8 RBB implementation assumes that the exposure-point monitors identify the sources of pollutants (i.e., pollutants released by the refinery and pollutants released by other sources) and that CREAM uses this information to develop risk estimates. 
taken, and accountability is ensured because the alternative measurement technologies must meet performance-based criteria. The ability to use cost-effective alternative measurement technologies provides an incentive to develop more efficient technologies.

\subsubsection{Risk-Based Exchange Issues}

A key element of the RBB is the ability to exchange or trade releases across pollutants and media on the basis of risk. Such exchanges help provide flexibility in meeting the environmental goals of human health and ecological protection as measured by acceptable risk. Primarily, exchanges are expected to occur within the refinery bubble. However, under certain circumstances, exchanges between the refinery and one or more nonrefinery sources may occur. The ability to exchange is based on the assumption that risk provides the measure (or currency) on which exchanges can be made. The revised RBB invokes a terminology change from the strawman RBB approach. The strawman approach referred to these exchanges as trades. In existing trading programs (e.g., the Acid Rain Trading Program), the currency is usually tons or dollars, neither of which captures any information with respect to toxicity, exposure route, population exposed, or other factors related to public health or the environment. However, the RBB currency - risk - does account for these factors and is therefore more appropriately considered an exchange. (The RBB exchange can be considered analogous to a diet exchange, where a certain amount of one kind of food is exchanged on the basis of nutritional and other health-based factors for another.)

CREAM accounts for cross-pollutant and cross-media exchanges. CREAM uses an agreedupon acceptable risk level as the "control total." It then runs various combinations of the refinery's releases to identify pollutant-specific release limits that will keep total cumulative risk within acceptable levels. The following paragraph gives an example to illustrate the RBB exchange concept.

A refinery may have one or more pollutant releases that are particularly difficult to reduce. At the same time, the refinery plant manager may have information indicating that a new process, catalyst change, or pollution prevention technique could reduce emissions of one or more other pollutants at the refinery. In such a case, the plant manager would estimate the media-specific quantities of releases of the pollutants after applying the process, change, or technique. These revised estimates would be input to CREAM, which would then calculate a revised total cumulative risk estimate on the basis of the revised pollutant-specific releases. CREAM would then identify combinations of acceptable risk-based release limits for the remaining contaminants, which the refiner and the RBB Board could review. The refiner could then select and implement a given RBB Board-approved combination of pollutant release limits. In this way, the total cumulative risk would remain acceptable, thereby protecting human health and the environment, and the refiner could meet environmental protection goals more cost effectively than without the exchange.

Because CREAM would account for and track uncertainty, the revised limits could be adjusted (made more strict) in cases where uncertainty exceeded a certain factor. The model could also adjust the allowable exchange to reflect the use of monitored versus modeled data, so that an exchange based on monitored data would be worth more than one based on modeled data. 
(CREAM would also provide details on emissions and assumptions used to generate each of the revised limits as well as information on the geographic areas in which the releases would be made. These details would be publicly available.)

The strawman RBB approach also provided for the banking of emissions or release credits. Thus, if the baseline releases from the refinery contributed to a total cumulative risk that was significantly less than the agreed-upon acceptable risk, the refinery could "bank" some of those release credits for use in the future when, for example, changing feedstocks led to increased emissions. The revised RBB does not provide for banking of releases for two reasons. First, because risk is measured at a specific point in time, it would be inappropriate to try to apply previously earned release credits to a current risk scenario. Also, the "cashing in" of banked release credits by multiple sources at some future date could result in "hot spots," where total cumulative risk was significantly greater than the acceptable risk level. If the total cumulative risk level at the time a refinery wanted to use its banked credit was greater than the acceptable risk level, the refinery would not be able to use that credit. It is possible that the refinery could never use the credit, which could raise issues of fairness and possibly of "regulatory takings," if credits were considered property rights and were never allowed to be used.

During the workshops, several participants questioned the use of trading. The following paragraphs summarize specific concerns and describe how the revised RBB addresses them.

\subsubsection{Cross-Pollutant Trading}

Most participants supported the concept of allowing the refinery to trade releases of a given pollutant among various sources within the refinery bubble. Most also agreed that the same pollutant could be traded across media, within the bubble. However, several participants opposed the concept of cross-pollutant trading regardless of where it occurred. They suggested that different pollutants had different health and ecological impacts and that spikes of certain pollutants (e.g., those that were particularly difficult or costly to control) could result from trades that involved more than one pollutant. Because CREAM accounts for the risks of all pollutants to produce a cumulative total risk, any releases that lead to high risk would be reflected in the cumulative risk and therefore would not be allowed. (In the interim, as CREAM is developed, the maximum risk-based release limits would be enforced.)

\subsubsection{Pollutants Subject to Trading}

Some participants cautioned that trading may be appropriate for criteria air pollutants that can travel long distances, such as $\mathrm{SO}_{2}$, particulate matter, and $\mathrm{NO}_{\mathrm{X}}$. However, they suggested that some pollutants, such as toluene and benzene, have particularly high impacts on public health and the environment near the emission sources. Therefore, while trades may be possible from within the same source, off-site trading of these pollutants should not be allowed. Some said that a lack of adequate exposure data and scientific studies on human health effects means that a better understanding of the health effects of these pollutants is required before trading should be implemented. Others said that certain pollutants should not be traded under any circumstances, because there is insufficient information to develop equivalent trades. Still others said that because the public considers some pollutants to be very harmful, attempting to trade such 
pollutants would provoke negative reactions. The project team recognizes that more information is needed before the approach can be implemented, but it maintains that as such information becomes available, it will be appropriate to allow for exchanges, as long as the health effects are understood and the total cumulative risk remains acceptable. In the interim, adjustments can be made (more stringent emissions limits) to account for uncertainties.

\subsubsection{Geographical Considerations}

The harm caused by a given pollutant may be greater in one geographic area than another. Similarly, because air is cleaner in some areas than others, emissions reductions generated in areas with clean air should not be used to allow emissions increases in areas with more polluted air. Because CREAM accounts for environmental conditions, the revised RBB contains a built-in mechanism for avoiding such unequal trades.

\subsubsection{Trading as a Purely Economic Incentive}

Some stakeholders said they viewed trading in the traditional sense (i.e., the ability to buy the right to release a ton of $\mathrm{SO}_{2}$ at one facility and provide for an equivalent reduction at another facility) as a purely economic incentive, which does not contribute to overall reductions in pollutant releases or improvements in public health. The revised RBB approach does not trade on the basis of tons but rather on the basis of risk. Thus, it accounts for the fact that a ton of $\mathrm{SO}_{2}$ in one region may result in a greater risk when released from a facility directly upwind of a large population center than a ton released from a facility where there is much less potential for exposure. Therefore, the revised RBB provides for the improvement of environmental conditions (or the maintenance of environmental conditions that are already considered acceptable).

\subsubsection{Environmental Considerations}

In 1992, the South Coast Air Quality Management District in California approved a program in which five oil companies earned emission credits by buying and scrapping old, highly polluting cars. The credits allowed the companies to forgo emission controls on tankers that load and unload fuel. However, two local citizens groups sued the oil companies, charging that while

the trading program reduced smog levels in the entire Los Angeles region, vapors released by the tankers directly affected only one small community (Wood 1997). The RBB exchange system would prevent such occurrences, because it accounts for local impacts and ensures that exchanges are made on a risk-equivalent basis. To provide additional assurance that exchanges result in equivalent risk, potentially controversial trades should be discussed with input from potentially affected parties and approved by the RBB Board. The EPA noted that economic incentive programs, such as trading, have the potential to cause unacceptable local increases in toxic emissions. They can also provide options to mitigate that potential, which include the following (EPA 1999g):

- Restrict actions that would increase releases to above pollutant-specific de minimis levels. "In this option, the state or locality could establish acceptable increments of risk ... and could presumably establish de minimis emission rates based on a simple modeling analysis of the emissions level that would typically cause the concentration for 
each pollutant that would be estimated to cause the acceptable increment of risk to be exceeded."

- Conduct a site-specific analysis for each prospective trading activity. Recognizing that the costs to do so could be high enough to limit trading activity, the EPA suggests that analysis be required only for emission increases of specified hazardous air pollutants.

- Establish zones, with inequitably high risks, in which trades of hazardous air pollutant emissions would be prohibited.

- Disallow activities that increase emissions of "very hazardous toxics." Very serious toxics would include hazardous air pollutants that are "potent carcinogens or for which long- or short-term exposure to low concentrations can cause serious noncancer effects."

- Require activities that allow the emission of a hazardous air pollutant to increase to also require a compensating equal-mass decrease of an emission of a hazardous air pollutant that is equally or more toxic.

The revised RBB incorporates each of these options to some degree via CREAM and the RBB Board review and approval process.

\subsubsection{Performance Evaluation}

Various government and nongovernment organizations emphasize the importance of performance evaluation. For example, in its final report, Framework for Environmental Health Risk Management, the Presidential/Congressional Commission on Risk Assessment and Risk Management said that "evaluation is critical to accountability and to ensure wise use of scarce resources" (Presidential Commission 1997). Similarly, of the 10 recommendations offered by EPA's SAB in its May 1999 report on integrated environmental decision making, two pertain to performance evaluation (EPA 1999e). Thus, the SAB urges the EPA to "identify, collect, and disseminate scientifically based environmental metrics organized in new ways to support a more integrated approach to managing environmental risks." It also recommends that the EPA develop a system of "report cards" to provide information on the status of human and ecological health and quality of life to assess the effectiveness of environmental decisions and to guide future environmental management decisions.

Several workshop participants also stressed the need for alternative regulatory approaches to provide for performance measurement. They identified two types of measurement needs. The first uses environmental data and information to determine the effects of regulatory programs on public health and the environment. These environmental indicators are measured or observed values that provide information on environmental conditions or trends in the area of potential refinery impact. Several organizations (e.g., World Resources Institute) are working to develop environmental indicators to measure such conditions, or outcomes, on local, regional, national, and global scales. The second type of performance measure indicates the success or effectiveness of the alternative regulatory approach in meeting its goals (i.e., for the RBB, ensuring that total cumulative risk does not exceed acceptable levels.) 
Reflecting the needs expressed by stakeholders and other organizations, the revised RBB provides two types of performance evaluation. As noted, the revised RBB strives to improve public health and the environment by requiring refineries to control emissions such that the total cumulative risk to public health and the environment is within agreed-upon, acceptable risk levels. Because this approach links releases to health and ecological benefits, it can be argued that by design, the RBB contains a built-in environmental indicator. However, today, the link between releases and risk depends on a number of assumptions. It is possible that measures other than risk could show direct links between release reductions and health and environmental benefits, without relying on assumptions. Thus, the first set of indicators measures environmental conditions linked to refinery releases.

Examples of such environmental indicators include the following:

- Unhealthy air events;

- Local disease and hospitalization rates linked to refinery emissions;

- For specific pollutants, the ratio of the actual concentration to the acceptable concentration, where the acceptable concentration is calculated by using the acceptable risk levels;

- Streams and rivers fully supporting aquatic life uses;

- Fish killed by pollution and fish tissue advisories;

- Plant and animal diversity;

- Stream miles open to migratory fish; and

- Percentage of native plants, fish, and wildlife species that are endangered, threatened, or of special concern.

Many environmental indicators require data that would not necessarily be collected by the refinery (e.g., health and ecological conditions, baseline and surveillance information about disease incidence rates linked to environmental causes). Thus, meaningful application of environmental indicators will be enhanced by the extent to which such data are collected and made publicly available. Recent efforts by EPA headquarters offices and other organizations to increase data collection and dissemination will hasten the development and use of meaningful environmental indicators.

The second type of indicator - the performance indicator - measures the effectiveness of the RBB in meeting its goals. While the critical measure from an environmental perspective is the actual benefits to human health and the environment, the critical measure from a performance perspective is how efficiently and effectively an approach meets those goals. For example, if an approach is too costly, or if stakeholders continue to feel excluded from providing input to the decision-making process, that approach will not be effective. Recent GAO testimony criticized 
the EPA's regulatory reinvention activities because they displayed an uneven record in evaluating the success of the EPA initiatives (GAO 1997b). Similarly, refinery workshop participants involved in the Yorktown Pollution Prevention Project noted that failing to establish and include performance measures in the design of that project limited its success (Klee and Podar 1992). Performance indicators could be used to help identify components or elements of the revised RBB that need improvement.

Examples of performance indicators include the following:

- Community satisfaction;

- Number of refineries that have adopted the revised RBB approach;

- Reduced amount of time and effort devoted to environmental compliance (by refineries, regulators, and the local community);

- Incorporation of public concerns into decision-making processes;

- Diversity of views represented;

- Improved understanding of alternative viewpoints and interests;

- Increased understanding of scientific underpinnings;

- Greater public access to information;

- Better working relationships and increased trust;

- Reduced amount of litigation;

- Cost savings realized by the refinery and regulator (on the basis of total cost accounting techniques);

- Impacts on civil rights/environmental justice issues;

- Increased number of positive and/or decreased number of negative news reports about the refinery;

- Increased use of science and data in setting limits and/or reduced use of assumptions;

- Reduced amount of environmental fines and penalties; and

- Positive media publicity about the program or community.

The RBB Board should develop both types of indicators by using generic information from entities that develop such indicators and site-specific input from local stakeholders. The RBB 
Board should consider the following factors in developing environmental and performance indicators:

- Uses and users of the indicators. The RBB Board should identify who will use the indicators and how they will be used. For example, the RBB Board may use environmental indicators to assess whether the risk-based limits need to be adjusted. Others may use the indicators to compare the performance of the revised RBB with that of other regulatory alternatives or to set priorities in other areas of environmental protection. Similarly, the RBB Board may use performance indicators to assess program operations and identify areas for improvement, and local community groups may use these indicators to gauge the degree and nature of public participation. Stakeholders may use performance indicators to determine whether actions were successful, identify lessons learned, and identify information gaps. The indicators should measure outcomes rather than procedures.

- Timing of evaluations. Evaluations may need to measure short- and long-term impacts. Initial measures may focus on progress in implementation of the program, with later evaluations directed toward the success of the program in meeting environmental risk reduction targets. The RBB Board may want to consider indicators that can identify trends in environmental changes.

- Data availability. The RBB Board should consider the kinds of data available for developing the indicators, but it should not eliminate potentially good indicators solely because needed data are not currently available. Indicators should relate to identified environmental issues and goals, not necessarily to available data. Recent advances in data collection and management techniques indicate that the amount, nature, and accuracy of data will increase over time. In addition, some environmental indicators may help identify and communicate data gaps, prompting actions to fill them.

- Communication to intended audience. All indicators should be explicit and well documented, so that users can understand how they were derived. As should other information reported on the revised RBB, the indicators should be reliable, accessible, meaningful, and transparent.

- Presentation. As visualization techniques are developed, the RBB Board should consider using graphic indicators in addition to explanatory and numerical indicators. For example, information on exposures could be related to effects by using geographic mapping systems.

- Number of indicators. The project team suggests that the RBB Board select or develop a relatively small number of indicators. A limited number will help maintain simplicity in data collection and interpretation and provide a focus for measurement efforts.

- Flexibility to change as needs change. Many factors (e.g., public comment, research, additional options) could clarify or redefine the goals and objectives of the program. As 
such, the RBB Board should be prepared to use an iterative process in developing and modifying the indicators to maintain flexibility and responsiveness to changing needs.

- Leveraging of other efforts. The RBB Board should avail itself of indicator development efforts underway in organizations such as the ISO, Environmental Council of the States (ECOS), and EPA to assist in its development of meaningful environmental and performance indicators.

\subsection{RBB Implementation Issues}

The project team and workshop participants identified a variety of concerns associated with implementing the RBB, particularly in the near term. As noted, the RBB is not intended to be implemented immediately; thus, many of the issues should be resolved as part of the overall regulatory reinvention process. Section 5.7.1 reviews such issues. However, implementation constraints caused by existing federal and state laws and regulations may require Congressional action. The following subsections first review issues that can be addressed by ongoing regulatory reinvention efforts and research, then review issues that may require changes in existing federal and state legal requirements.

\subsubsection{Issues Addressed by Ongoing Improvements in Science, Technology, and Institutional Change}

The following paragraphs highlight concerns that could impede implementation of the RBB in the short term.

\subsubsection{Technology Requirements}

Because the RBB is designed for future implementation, many of the provisions assume the use of technologies that may not have been demonstrated or may not be widely available commercially. Refinery representatives noted that suitable implementing tools, particularly for monitoring and modeling baseline and compliance activities, would facilitate acceptability of the RBB. As noted above, fence-line and exposure-point monitors require further development and commercialization to become cost effective. However, most participants agreed that such monitors will be commercially available, and that alternative regulatory programs such as the $\mathrm{RBB}$, which require such monitors, will help reduce the costs and improve the technology of fence-line and other exposure-point monitors.

\subsubsection{Setting Risk-Based Limits}

Theoretically, risk-based limits can be calculated for any pollutant, assuming the data pertaining to toxicity assessment, hazard assessment, dose-response relationships, transport mechanisms, and so on are available. Today, much of this information is not available. However, advances in data collection, management, and analysis, combined with calls for increased use of risk assessment by a variety of government and nongovernment organizations, should drive the development of such data, so that the ability to set such limits will become more practical over time. Nonetheless, prior to implementation, the availability and accuracy of the data for each 
residual for which limits would be set must be determined. Uncertainties and data gaps must be identified, addressed, and documented.

\subsubsection{Institutional Impediments to Multimedia Approaches}

Federal and state environmental regulations reflect the single-medium laws from which they are derived. Often, regulatory offices that are concerned with just one environmental medium have difficulty implementing multimedia approaches, because employees remain accountable for meeting the requirements of medium-specific laws (GAO 1997c). EPA workshop participants noted that many agency staff have not promoted multimedia approaches because the lack of information on multimedia interactions leads stakeholders to attack EPA's science, which, in turn, undermines the agency's credibility. Texas regulators said they were willing to consider innovative regulatory approaches but expressed frustration over the unwillingness of the EPA to embrace innovation. Noting that "programs, regulations, and procedures developed under current statutes often preclude an integrated approach," the Presidential/Congressional Commission on Risk Assessment and Risk Management offered several recommendations to overcome such impediments (Presidential Commission 1997). For example, it recommended that Congress coordinate the activities of committees with overlapping or related jurisdictions and competing responsibilities to reinforce integrated approaches for reducing risks in industrial sectors and geographic areas.

\subsubsection{Resource Requirements}

Establishing baselines and developing the modeling system that will set the risk-based limits and monitor compliance will require significant time, effort, training, and money. However, data collected under other auspices should facilitate implementation of the RBB, and such activities will not be the sole responsibility of the parties involved at a particular refinery undertaking the RBB. Some citizens group representatives suggested that interim activities such as increased education on risk, health effects, and related topics will facilitate future implementation.

\subsubsection{Nontechnical Issues}

Workshop participants raised several nontechnical issues that can impede implementation. These include the following:

- Reluctance to change. Many stakeholders are concerned that changing the current system will set precedents that could "unravel" improvements and requirements that have been instituted only after years of hard work.

- Fear offlexibility. Some refiners and regulators are more open to change than others. Corporate-level personnel may be more likely to favor a flexible approach than plantlevel personnel, who may prefer conventional, technology-based requirements. Even if flexibility is allowed, a plant manager may resist investing resources into a new approach that regulators may not approve. As one regulator stated, "Lots of people have bought into the box that we have." 
- Public acceptance. Many of the RBB elements have not been tested. Lack of experience with risk-based tools and possible biases against their use suggest that interested parties must become more knowledgeable about the RBB concepts and approaches before they are implemented. Educational briefings, meetings, focus groups, and other techniques designed to share information and gain public acceptance will be required.

The above implementation issues are not trivial. However, current trends in regulatory reinvention, stakeholder participation, multimedia approaches, technology development, enhanced communication, increased sophistication with risk assessment and other models, and expanding capabilities to collect, analyze, and disseminate data, combined with the fact that government and nongovernment entities recognize that the current system must become more integrated and results oriented, will address many, if not all, of these issues and thereby facilitate implementation of the RBB. However, implementation constraints caused by existing federal and state laws and regulations may require Congressional action. These concerns are discussed in the remainder of this section.

\subsubsection{Issues Addressed by Legislation}

State laws typically reflect federal legislation. Thus, federal statutory requirements that could conflict with the elements of the RBB are discussed first. Then state-level issues and efforts that could facilitate implementation of the RBB by providing regulatory flexibility are described for Texas, a state with several refineries and a progressive regulatory agency. Because none of these state-level initiatives is designed to usurp any federal laws, none is sufficient to allow immediate implementation of the RBB.

\subsubsection{Federal Statutes}

This section summarizes (1) sources of potential inconsistencies between RBB provisions and federal laws, (2) specific statutory provisions that could limit implementation of the RBB approach, and (3) options for statutory modification.

1. Sources of potential inconsistencies. The following five areas may lead to potential inconsistencies between the RBB and current statutory requirements:

- Multimedia approach. The RBB uses a multimedia rather than a single-medium approach to environmental regulation. Congress wrote laws to address problems with individual media, but there are few, if any, provisions to address cross-media impacts or release limits. Specific multimedia provisions that may be incompatible with existing laws include prioritizing releases for setting release limits, using a multimedia permit where existing laws contain permit requirements that would not be met by a multimedia permit (e.g., specific monitoring requirements), and trading of releases across environmental media.

- Standards development process. The revised RBB requires regulators, refineries, and citizens groups to develop risk-based release limits. To do so, these stakeholders will need to apply their knowledge to evaluate the environmental conditions into which 
emissions and effluents will be released. However, existing statutes can limit stakeholder participation of this type. For example, the courts have interpreted the CWA as not allowing the condition of the receiving water to be considered when setting effluent limitation guidelines. Such a consideration would be necessary for developing release limits under the revised RBB.

- Basis for setting release limits. The RBB uses risk as the basis for setting release limits. In contrast, many existing statutes use technologies or other factors for setting release limits. For example, the standards of performance for new stationary sources under the CAA are to reflect the degree of emission limitation that is achievable by applying the best system of emission reduction that has been adequately demonstrated. Similarly, the CWA allows the EPA to require more stringent riskbased standards to help ensure protection of public health and the environment for cases in which technology-based standards may fail to do so. However, the CWA does not allow the use of less stringent risk-based standards, even if they sufficiently protect public health and the environment.

- Incorporation of pollution prevention. The RBB relies on pollution prevention as a means for achieving environmental protection goals and includes incentives to promote pollution prevention. Many statutes lack pollution prevention incentives. For example, the CAA allows limits to be set for a source or an entire facility. However, few, if any, incentives exist to reduce emissions from sources that are not regulated.

- Compliance assurance. The approach requires flexibility for ensuring compliance beyond that available in existing statutes. Because some of the provisions are inconsistent with existing statutory and regulatory requirements, refineries operating under these provisions could be subject to administrative orders and civil and criminal penalties. Similarly, citizens can sue the EPA for failing to comply with statutory requirements.

2. Statutory provisions. Congressional legislation requires the EPA to write rules implementing medium-specific statutory requirements. In response, the EPA has organized activities and established program offices to reflect the environmental media and substances covered in the laws. Today, numerous individual statutes govern the EPA's activities. Implementing the RBB could breach specific statutory provisions in the CAA, CWA, and RCRA that relate to permits, schedules, enforcement, and requirements for technology-based standards.

In addition, these statutes and their implementing regulations, which have evolved independently of other statutes and regulations, are not well coordinated or integrated. No legislative or regulatory provisions consider the relative importance and priorities of various statutory objectives, deadlines, schedules, and standards. Furthermore, existing statutes do not authorize the EPA to relax certain requirements under one law in return for control beyond the statutory requirements of another law. For example, although relaxing a particular effluent limit in return for a site-specific waste management 
initiative could produce a net reduction in overall adverse public health impacts, existing individual statutes do not provide for such tradeoffs.

Concerns over statutory requirements can limit the ability and desire of state regulators to experiment with integrated, multimedia environmental management programs. As long as regulatory personnel are accountable for meeting the requirements of the federal laws, they are not likely to foster approaches that deviate from established protocols.

3. Statutory modification options. Three options were identified for mitigating statutory implementation issues. These options are summarized below and are compared in Appendix G.

- Pilot-study waivers. The simplest of the three options would be to include a provision in an appropriations bill specifically authorizing the EPA to waive environmental requirements necessary to implement the RBB on a pilot scale. Such an amendment would cite examples of the types of requirements to be waived and would allow other regulations to be waived as necessary. Time limits for the provision, restrictions on the number of refineries to which the provision would apply, and a statement that the waiver is not designed to set a precedent would be included. This technique has been used in the past; Congress frequently attaches provisions to appropriations bills to foster quick passage. Drafting such an amendment would be relatively simple, and chances for passage of an appropriations bill would be better than for a standalone bill (because appropriations bills must pass). The Presidential/Congressional Commission on Risk Assessment and Risk Management recommended that Congress "explicitly authorize EPA and state agencies to enter into compliance agreements that waive certain current regulatory requirements if alternative controls can credibly achieve equal or, whenever feasible, greater environmental protection" (Presidential Commission 1997).

- Strategic waivers. Broader than pilot-study waivers, strategic waivers would allow the EPA to waive compliance for any number of refineries (or other entities) on which the EPA, the regulated entity, and other stakeholders could agree. The vehicle would be a standalone bill that would make the waiver available to any facility as long as appropriate criteria (to be developed by the EPA) were met. There is a precedent for this type of waiver. In October 1997, Sen. Joseph Lieberman (D-CT) introduced a bill (S. 1348) that would authorize the EPA to approve proposals for experimental projects seeking regulatory flexibility in exchange for superior environmental performance. The bill effectively grants legislative approval for Project XL agreements as long as significant public participation by all stakeholders has occurred and several specific factors have been considered. These factors include how the strategy prevents pollution, reduces risks, improves environmental conditions that are priorities to affected parties, addresses community and health priorities of the public, and results in environmental performance that is more protective than the best performance practice of comparable facilities. Rather than pertaining specifically to Project XL, strategic waivers would apply to the revised 
RBB more comprehensively. Criteria would differ from those outlined in S. 1348, but the concepts in S. 1348 and the strategic waiver option are similar. Strategic waivers would be broader and could take much more time to implement than pilotstudy waivers because of the processes needed to introduce, debate, and pass such legislation.

- Individual statutory changes. This option would make permanent changes to individual environmental statutes (e.g., CWA, CAA, RCRA), enabling implementation of a number of regulatory reforms such as those contained in the revised RBB. Although most interested parties recognize the need for regulatory reform, the effort required to analyze each statute to identify needed changes and their implications, combined with the need to build support for such changes, could make the effort unachievable in the near term. Also, statutory changes of this sort could have major ramifications on operating practices in a number of industries, and support for such far-reaching changes may not be extensive.

\subsubsection{State-Level Initiatives}

As noted, federal environmental statutes extend to the state level, and while state rules can be more stringent than federal rules, they cannot be less stringent. Recognizing that state regulations can limit flexibility and inhibit innovation to improve environmental conditions at reduced costs, several states are developing regulatory reform programs to provide for such incentives. While these programs can provide flexibility (e.g., facilitywide permitting with a total cap on emissions), federal statutory constraints remain. The remainder of this section describes potential RBB implementation issues and regulatory reform initiatives underway in Texas.

Most Texas environmental regulatory programs are not considered more strict than their federal counterparts. However, some Texas programs have no corresponding federal program. For example, all new sources in Texas must be permitted by exemption, by a standard permit, or by a case-specific permit. At the federal level, only major sources (those emitting 100 tons per year or more of any pollutant) require permits. Sometimes, the Texas legislature will incorporate an EPA regulation into a state statute. For example, every rule that pertains to an air quality state implementation plan must be submitted to the EPA, which adopts it and publishes it in the Federal Register. Thus, before RBB implementation, both the federal statutory issues and the state rules and the contracts and agreements between the state and the federal government would need to be considered.

General procedural requirements could affect implementation of the RBB in Texas. For example, the Texas model for public notice for permitting and enforcement differs from the national model. In Texas, all permit hearings are contested case matters; that is, each case requires a trial. The federal model is based on notice and comment, reflecting an administrative law approach. Because a "permit hearing" in Texas requires months of intensive regulatory staff preparation time, the preparations required to implement the RBB could also be significant.

Despite these implementation issues, Texas is developing some regulatory initiatives that could facilitate RBB implementation. Four such initiatives are described below. 
1. Regulatory flexibility. The Texas Natural Resources and Conservation Commission (TNRCC) has recognized that while existing environmental laws and regulations play critical roles in protecting public health and the environment, such protection could be enhanced by implementing innovative environmental regulatory methods. It has sought to promote regulatory methods that do the following:

- Encourage facility owners and operators to innovate, set measurable and verifiable goals, and implement the most effective pollution reduction strategies for their particular facilities, while complying with verifiable and enforceable pollution limits;

- Reduce time and money spent by agencies and facility owners and operators on paperwork and other administrative tasks that do not benefit the environment; and

- Increase public participation in the development of innovative environmental regulatory methods and in monitoring the environmental performance of projects under the program.

In 1997, the Texas legislature authorized the TNRCC to issue regulatory flexibility orders (RFOs) to applicants requesting exemptions from Texas statutes or TNRCC rules regarding the abatement or control of pollution (30 Texas Administrative Code 90.)

To obtain an RFO, applicants must summarize their proposals, including the specific statutes or rules for which an exemption is sought; explain how the proposed alternative is at least as protective of the environment and public health as the method or standard that would otherwise apply; provide an implementation schedule that addresses monitoring, record keeping, and reporting; identify transfers of pollutants between media; and explain how the local community is or was involved in developing the proposal. The program addresses noncompliance with and amendments to the RFO.

2. Flexible permits for air. The flexible permit rule, which became effective in July 1998, provides facility owners and operators with flexibility in how they meet established emissions limits. According to the rule, if all of the individual units at a facility have installed best available control technology (BACT), and they are meeting the total emissions goal associated with the BACT, then the owner/operator can opt for a plantwide emissions cap. Caps are established on a pollutant-specific basis, by calculating the emissions from each source based on application of BACT, and then summing the emissions over all sources. The cap allows facilities to change throughput or feedstock as long as additional steps are taken to ensure that overall emissions do not increase.

The flexible permit rule allows a plantwide cap on emissions. However, because it requires that the emissions limits be set on the basis of BACT and not on the basis of risk, it would need further refinement before it could help in implementing the RBB. 
3. Texas Risk Reduction Program (TRRP). The TNRCC administers several sets of corrective action regulations under the Superfund, Voluntary Cleanup, Petroleum Storage Tank, Industrial and Hazardous Waste, and Underground Injection Control programs. The rules under these various programs contain different and, in some cases, contradictory regulatory approaches, requirements, and cleanup objectives. As a result, industry, the public, and the regulators must learn, implement, and comply with different regulations, and the results - under comparable circumstances at the same site - can be inconsistent.

The TNRCC has been developing the TRRP for several years. It adopted an early version in 1993, and in 1995 it adopted a risk-based petroleum storage tank rule. Since then, continued advances in science have extended the scope of those rules. The current version of the TRRP incorporates new, nationally developed corrective action methods. The TNRCC believes that by incorporating updated standards in risk reduction, the new TRRP rule will improve protection of human health and the environment, while enhancing flexibility and containing costs for the regulated community.

The goals for the new TRRP include the following:

- Create a unified, performance-based approach to corrective action;

- Establish risk-based corrective action for waste-related programs that do not fall under federal regulations;

- Establish a program that other agency programs would reference when developing corrective action programs; and

- Implement a consistent, streamlined approach to expedite remediation of affected properties.

The new program allows owners and operators to make their own cost-effective decisions, while ensuring that the response action is protective of human health and the environment. It uses a three-tiered approach that incorporates risk assessment techniques to set (1) protective concentration levels in environmental media and (2) reasonable response objectives for protecting human health and the environment. The first tier, which uses generic assumptions in the risk assessment, is the most conservative. The second and third tiers allow owners and operators to incorporate site-specific data into the risk assessments to increase protective concentration levels and determine appropriate response actions. This flexibility in investigation versus remediation allows owners and operators to adjust the relationship between investigation costs and remediation costs and helps achieve the lowest combined costs. In addition to standardizing the corrective action process, the program also provides for a facilitywide approach to recognize the complexity of and accommodate the need for continuing operation while managing corrective action at large operating facilities such as refineries. 
The TRRP initiative sets several significant precedents for implementing the RBB. It recognizes the need for flexibility while protecting public health and the environment, and it strives to reduce the redundancy and duplication found in several environmental regulations that often cover the same contaminant in the same media. Moreover, it sets a clear precedent regarding the acceptability of and need for using risk-based standards to help ensure the cost-effective protection of public health and the environment.

4. Permitting of grandfathered facilities. Representatives of several citizens groups explained that the current regulatory structure often exempts certain facilities from having to comply with laws and regulations. As a result, regardless of how strict such laws and regulations may be, the public health and the environment can still be negatively affected by these "grandfathered" refineries (and other facilities). In Texas, facilities operating before 1971 are exempt from the Texas New Source Review Permitting program. Thirty refineries in Texas have grandfathered emissions (i.e., emissions not subject to New Source Review). On average, between one quarter and one third of air emissions from these refineries are exempt. At some refineries, virtually none of the emissions are subject to control.

In 1996, the Texas legislature directed the TNRCC to develop a voluntary emission reduction plan for permitting existing significant sources. The TNRCC appointed an 11member advisory committee - the Clean Air Responsibility Enterprise (CARE) Advisory Committee - to develop recommendations for achieving this goal. The committee met several times and prepared a concept paper that recommended incentives such as tax abatements and emissions trading. It also recommended that a facility seeking a CARE permit undergo an "abbreviated health-effects review." The TNRCC recommends that such a review examine the following three elements:

- Whether the facility is proposing to use current BACT;

- Whether the facility is in a remote location and is at an acceptable distance from the nearest receptor; and

- Whether existing monitoring data can demonstrate that there will be no off-site impacts, or whether the facility proposes to use fence-line or stack monitoring technology to demonstrate ongoing protection of public health.

If a facility failed to demonstrate protection of health by not having at least one of these elements, it would undergo a qualitative health-effects review that would examine toxicity and volume of emissions in light of the meteorology and nature of the area surrounding the facility.

The TNRCC held hearings on these recommendations throughout the state and incorporated comments into the plan. It is now developing final rules, which are expected to address the application process, control technology requirements, public participation, and the review of health effects. The CARE program is consistent with the 
revised RBB approach of identifying all sources of releases and basing compliance requirements on local health considerations.

Facilitating the implementation of regulatory flexibility in Texas is the TNRCC's formal recognition that reform is needed. In April 1996, the TNRCC established a regulatory reform process to review its rules to "ensure that they are as clear and simple as possible, give the maximum flexibility to our staff and the regulated community to enable them to reach our environmental goals, do not impose burdens without producing corresponding benefits, and follow legislative direction" (TNRCC 1996). The process uses several ad hoc teams under the direction of a Regulatory Reform Committee and representative outside parties to work on reforms ranging from immediate targeted fixes to more long-term and far-reaching reforms. Ideas for regulatory reform are solicited from the staff and outside parties. The TNRCC considers the regulatory reform process to be "one of the agency's top priorities" (TNRCC 1996). 


\section{Section 6 Revised NPA}

As discussed in Section 4.2.3, workshop participants asked for clarification, additional explanation, and details on many of the elements that made up the NPA strawman approach. They also stressed that all three components of the NPA needed to embody meaningful public participation. Some suggested improving the NPA structure by adding an initial component that would establish refinery-specific NPA goals. Many of the workshop comments pertained to both the RBB and the NPA, and both revised prototypes address several of these issues and concerns in the same way. The following discussion of the revised NPA does not reiterate the details of such modifications. Rather, it notes where the revisions to the NPA are the same as those to the $\mathrm{RBB}$ and provides references to appropriate sections in the revised RBB discussion.

Lessons learned from developing and, in some cases, implementing ongoing efforts that reflect NPA elements have been incorporated into the revised NPA, and examples of such applications are included in the discussion of the revised NPA. Because the revised NPA serves to establish a basic framework for developing and implementing a site-specific negotiated agreement, participating stakeholders will work together to provide details on many of the aspects to be included in their facility-specific NPA.

\subsection{Overview}

The NPA is a goal-based, facilitywide, multimedia regulatory program. The facility-specific baseline of residuals and environmental management costs reflects current operating conditions. It provides a starting point for setting release limits and a benchmark for measuring progress toward meeting those limits. Release limits are negotiated on the basis of goals established by state or federal environmental policies or by the parties involved in negotiations and on the basis of current and anticipated regulations.

Release limits are specified in terms of total allowable residual-specific releases (e.g, 500 tons of $\mathrm{SO}_{2}$ emissions per year) or in terms of residual-specific percentage reductions from baseline conditions. Release limits are to be developed so that existing and potential future regulatory limits will not be exceeded. The negotiated limits provide the basis for the negotiated agreement, which serves as a facilitywide, multimedia permit.

Because limits are negotiated separately for each refinery (as opposed to the more traditional "one-size-fits-all" approach) the limits can reflect actions that the refinery may have already taken to reduce releases below required regulatory limits. Limits are negotiated on a facilitywide rather than a source-specific basis, much the same as they are in the RBB. However, whereas the RBB allows for trading across environmental media, the NPA calls for mediaspecific limits. In the context of the NPA, the term multimedia refers to the type of permit. In other words, contrary to the current situation in which refineries must obtain several permits for 
numerous pollutants released to various media, the NPA requires only one permit, and that permit contains total allowable release limits for all pollutants to all media.

The permit remains in effect for a negotiated period of time, expected to be at least 10 to 15 years. At the end of the period, the refinery would be expected to have met its release reduction goals. To provide for continuous improvement, the limits could become increasingly stringent over time, and milestones could be set to assess progress.

Compliance in the revised NPA is assured via monitoring and reporting requirements, inspections of monitoring equipment, and a penalty system that includes both monetary and nonmonetary incentives and penalties. Penalties would be assessed for failure to meet reporting requirements, failure to maintain proper functioning of monitoring equipment, and failure to meet the release limits. The penalty system could be structured to reflect the number and nature of violations.

Many of the revised NPA elements are similar to those of other successful regulatory reinvention approaches that have been developed and implemented in the United States and overseas. In addition, the revised NPA reflects approaches recommended by various organizations as ways to improve the environmental regulatory system. Appendix H provides examples of efforts and studies that support the concepts an provisions of the revised NPA. The following examples provide support for the NPA concept.

\subsubsection{Project XL}

The EPA designed the Project XL (which stands for "eXellence and Leadership") regulatory reinvention program to encourage regulated entities to develop alternative environmental management strategies that exchange flexibility in how environmental responsibilities are met for "superior environmental performance" (EPA 1999i). Successful projects must demonstrate stakeholder support; provide for pollution prevention; establish accountability through reporting, monitoring, or other evaluations; and provide lessons that can be transferred to other facilities. Established in 1995, the program sought 50 pilot projects in the first year. As of August 1999, 14 projects had been implemented (EPA 1999i). The lessons learned from Project XL are directly applicable to the revised NPA and will be discussed in the following sections. Appendix I identifies several XL projects with provisions similar to those of the revised NPA.

\subsubsection{The Netherlands Covenants Program}

In 1989, the Dutch government published its National Environmental Policy Plan (NEPP), which changed how the government, business, and society in the Netherlands approached environmental protection. Designed to be a single, comprehensive, ecosystem-based policy to integrate all areas of environmental concern, the goal of the NEPP was to achieve sustainability within one generation, or 25 years. Planners developed eight themes or categories of environmental problems (e.g., climate change, waste disposal) and seven target groups (e.g., industry, agriculture) to help meet the NEPP objectives. Each target group consists of several sectors. For example, the industrial group includes primary metals, chemicals, and eight other sectors. For each sector, representatives of that sector and government officials negotiate a 
voluntary agreement, or covenant, which includes theme-specific goals for the sector to meet. It also includes timelines for achieving those goals and provisions for monitoring progress and developing contingency plans. Individual companies within each sector prepare their own company environmental plans (CEPs), which include measures to meet the goals and a strategic plan for implementing the company's environmental policy for the next four years. The company begins developing its CEP by inviting all its licensers (permitting agencies) for an initial meeting at which the licensers explain their minimum environmental requirements. Meeting participants examine the commitments in the sector's covenant and discuss how the company can help meet those commitments. The company then identifies its emissions and sources of emissions and develops a list of problems and potential solutions. After a series of negotiations between the licensers and the company, the company and the authorities sign the agreed-upon CEP, which forms the basis for the new license.

Because it does not contain detailed prescriptions, the license offers the company operational flexibility. However, the company is expected to develop internal management procedures and rules, and it must file clear and transparent reports. Once a company implements its CEP, it is exempt from other environmental regulations. If it fails to comply with its CEP, its regulatory system reverts to the traditional command-and-control approach. Results for the primary metals sector indicate that companies are introducing more process-oriented measures and are more open to incorporating new technologies. Generally, industry appears more enthusiastic about the program than government officials, some of whom argue that companies are not accepting their responsibilities. While the program has produced environmental improvements, it still must address several issues, such as judicial concerns, the roles of environmentalists (who at the beginning were not a part of the process, but who are now becoming more involved), and the fact that some existing requirements can hamper implementation of the new policy (Hofhuis 1999).

\subsubsection{Comprehensive State Permits}

Several states have begun implementing facilitywide, multimedia, or other comprehensive permit programs intended to reduce regulatory burden, increase operational flexibility, and provide equal or better environmental performance. Three examples are summarized below.

1. State of New Jersey Facilitywide Permit (FWP). The 1990 New Jersey Pollution Prevention Act authorized the state, under a pilot program, to issue FWPs for up to 15 facilities. An FWP is a single permit issued to an industrial facility that includes the facility's environmental requirements for air, water, and hazardous waste permitting and its pollution prevention plan. It allows a facility to change process equipment and raw material without the state's prior approval, provided it does not exceed process-level caps. The first step toward obtaining an FWP is for the facility to prepare a pollution prevention plan. The plan identifies the use, generation, and release of hazardous substances and nonhazardous pollutants for each production process in the facility. The facility then identifies pollution prevention opportunities, sets five-year pollution prevention goals, and selects strategies for implementing those goals. Once the pollution prevention plan is complete, the New Jersey Department of Environmental Protection (NJDEP) develops process-level limits and other conditions for all air, water, and 
hazardous waste releases on the basis of the facility's process-level accounting data (Spinello 1997). According to the NJDEP Office of Pollution Prevention, and Permit Coordination (OPPPC), the FWP program has demonstrated "benefits to the public from increased environmental and administrative efficiency. Prior to the advent of the facilitywide permitting program, existing single-medium permit programs inadvertently allowed risks from releases of hazardous substances to be shifted from one medium to another by transferring pollutants from air to water, air to land, or water to land. Through the process of drafting and issuing facilitywide permits, the Department has been able to identify, reduce, and control these releases and cross-media transfers, thereby benefitting the public" (NJDEP 1999d).

2. Oregon's Green Permits Program. In 1997, the Oregon Legislature created the Green Permits Program to encourage regulated entities to achieve environmental results that are "significantly better than otherwise provided by law" (Oregon 1999). Key principles underlying the Green Permits Program are the following: environmental performance must exceed minimum compliance requirements, measurable environmental performance goals must be established, meaningful stakeholder involvement is expected, and the system will balance "simplicity and certainty with flexibility and innovation" (Oregon 1999). The Oregon program requires that the permit include an environmental management system. Pilot facilities agree to analyze environmental impacts, set targets for reduced environmental impact, facilitate meaningful dialogue with stakeholders, develop an environmental report for public distribution, and propose incentives for the Oregon Department of Environmental Quality (DEQ) to offer to recognize superior environmental performance. Early results indicate improved environmental performance for all four facilities that have begun implementing the framework. The DEQ notes that experience gained at the facilities will foster efficient implementation once rules for the program are adopted (Oregon 1999).

3. Texas Clean Industries 2000. This is a voluntary pollution prevention partnership among industry, communities, and the TNRCC. Program participation is at the facility level, and all 180 participants committed to the following: (1) reduce TRI chemical releases at their facility by a minimum of 50\% from 1987 levels before the year 2000; (2) implement an internal environmental management program to assure high levels of compliance with state and federal standards; (3) participate in or establish a dialogue about the environmental planning and management in their area, community, and city; and (4) participate in one or more community environmental projects each year. The TNRCC reports that as a result of the program, waste generation has been cut by 8.7 million tons or 10.3\% between 1994 and 1996; TRI releases have decreased by 47\% between 1987 and 1996; and more than 500 community environmental projects have been established (TNRCC 1999).

While each of the above programs shares common elements with the NPA, none is identical in all aspects. For example, although XL projects require active public participation (as does the NPA), they tend to focus on exchanging superior environmental performance for relief from one or a few specific regulatory requirements. Thus, the scope of the NPA approach, which provides flexibility with respect to potentially all current regulatory requirements, is much broader than 
that of the Project XL program. The Netherlands Covenants program focuses on negotiation, target setting, and facilitywide agreements for all pollutants and all media, as does the NPA, but it also relies on a more top-down approach. Thus, the Dutch covenants are signed at the sectoral level rather than the facility level, and those covenants are tied to national-level goals. In the NPA, the goals are facility-specific, with no direct link to overriding goals established at sectoral or national levels. The state permitting programs generally share the concept of facilitywide permitting but rely less on public participation and negotiation than does the revised NPA. Other significant differences also exist. For example, the targets and limits in the New Jersey program are tied to chemical use rather than releases, and they require the facility to account for materials and set limits on a process-specific basis. The Oregon system requires an environmental management system, which the NPA does not. The Texas system, while it requires active public participation, does not appear to embody economic performance as a key component. Regardless of the differences in the particular facets of these programs, each provides support for the development and testing of the NPA. The following sections highlight how these efforts comport with the revised NPA.

\subsection{Public Participation}

As noted in Section 4, workshop participants expressed particular concern about the need for increased public participation in both the RBB and NPA strawman approaches. While the NPA strawman approach included collaboration between the regulatory agencies and the refinery in establishing the baseline and setting the limits, stakeholders expressed the need for a stronger, more active public role throughout the process. Numerous recent studies echo the need for and importance of stakeholder participation in environmental decision making (see Appendix $\mathrm{H}$ ). The revised NPA includes public participation all three components.

The breadth, depth, and impact of public participation in the revised NPA is expected to be the same as in the revised RBB system. However, the participation mechanisms will differ to reflect the differences between the two approaches with regard to technical concepts and degree of departure from the current system. The revised RBB, which more significantly departs from the current system than does the revised NPA, involves the implementation of such concepts as cumulative risk and risk-based trading across pollutants and environmental media. The revised NPA is much closer to the current system; the key difference between the revised NPA and the current system is that the limits are negotiated with stakeholder input rather than set by government agencies. The two-tiered revised RBB public participation approach facilitates implementation by distributing technical information collection and evaluation activities among participants who can then report back to the RBB Board, allowing the RBB Board to focus on oversight and decision making. Because the revised NPA requires less technical research and development than the revised RBB, a single NPA Council that includes public representation is considered to be the optimal approach for efficiently and effectively incorporating stakeholder participation throughout all components.

\subsection{NPA Council}

The NPA Council will be responsible for the success of the entire process, including forming the initial goals, setting the baseline, negotiating the release limits, and assuring 
compliance. As such, it determines and approves limits, enforces limits, and, if necessary, modifies limits. The NPA Council consists of representatives of the refinery; state, local, and federal regulatory agencies; and the local citizenry. It may also include representatives of the refinery's corporate offices, local elected officials, and national environmental groups.

NPA Council representatives are either voting or nonvoting members. The numbers of voting members and nonvoting members and the organizations represented will vary depending on the specific refinery. However, a suggested model would include 6 voting members (with membership akin to that of the RBB Board (i.e., two each from the refinery, the regulatory agencies, and the local citizenry) and 9 to 14 nonvoting members, for a total of 15 to 20 NPA Council members. This overall size is consistent with the recommended sizes of committees convened for similar purposes. For example, a 1995 Pennsylvania directive on regulatory negotiation recommended an optimal size of 15 to 20 members for negotiating purposes (Seif 1995).

As with the RBB public participation process, careful selection of NPA Council members is critical to allow for fruitful discussion and successful negotiations. All members must be willing and able to commit necessary time and effort to the process. They must be flexible, willing to negotiate, and knowledgeable (or willing to learn) about refinery processes. Local community members should be selected by the communities they represent and should speak for broad community values, preferences, and concerns. The 1995 Pennsylvania directive also listed other factors to consider when convening negotiating bodies. These included the following:

- Each stakeholder group represented must have an adequate incentive to negotiate an agreement in a timely manner.

- All parties must feel they will gain something by negotiating.

- No fundamental values can be in conflict.

- Multiple issues are subject to negotiation (so that tradeoffs are possible).

The NPA Council should incorporate these recommendations.

The voting members of the NPA Council would be responsible for decisionmaking, with input from nonvoting members. Consensus is not viewed as necessary within the Council, and conflict is to be expected. As with the RBB Board, decisions could be made by requiring agreement from at least four of the six members. The mix of nonvoting members should balance significantly affected stakeholder groups, including not only public participants but also additional refinery and regulatory representatives. A separate committee or experienced facilitator may assist with the selection process. Even with careful selection, the public participation and negotiating processes can involve failures, and initial expectations should not be high. The goals of the public participation process are cooperation, open communication, and understanding. Many workgroup participants noted the need for trust among various interest groups. As noted in a report on the Dutch covenants process, trust need not exist at the beginning of the negotiations, but it must develop over time. Regarding the relationship between industry 
and the regulators, the Dutch found that "once the initial mistrust had been overcome, both sides showed themselves willing to concentrate on the main point, the ultimate objective: how to achieve very specific reductions in emissions" (Pronk and Blankert 1999).

The revised NPA envisions a kick-off meeting focusing on process rather than technical details. At this meeting, the participants, with the aid of a neutral and experienced facilitator, should discuss schedules, roles, responsibilities, and priorities and agree to a set of ground rules. The types of ground rules to be considered and other aspects of the public participation process (e.g., use of facilitators, role of consensus, and the use of studies on successful public participation approaches) are essentially the same as those described for the revised RBB in Section 5.3. The results of the initial meeting and all subsequent meetings of the NPA Council should be thoroughly documented and signed by all members. The NPA Council may wish to consult EPA's Project XL Stakeholder Involvement: A Guide for Project Sponsors and Stakeholders, which provides detailed information on implementing effective stakeholder processes (EPA 1999j). The following sections describe components of the revised NPA, which the NPA Council will oversee and implement.

\subsection{Establish Goals for the Revised NPA}

Responding to the suggestion that the NPA should include an initial goal-setting component, the revised NPA calls for the NPA Council to establish overall NPA goals before establishing the baseline. Goals can be expressed in such terms as types of pollutants to be targeted for reduction, environmental impacts to be mitigated, pollution prevention expectations, or anticipated new technology implementation to be accomplished via the NPA. Such goals will help focus the entire NPA effort. Thus, they will help in prioritizing activities necessary to establish the baseline and they will assist negotiators in prioritizing pollutants for which release limits will be set. They will also help guide refinery personnel as they implement alternative approaches to meet the negotiated limits. Goals can also be used to scale incentives and penalties for compliance assurance.

Goal setting is an integral part of the Dutch covenants program and, in fact, forms its basis. In that program, national-level goals are set in the NEPP - the single environmental policy that applies throughout the country. The industry target groups and the sectors within those groups use those goals in developing the sector-specific covenants. Thus, when an individual company within a sector begins to develop its own CEP, it refers to the goals already set at the higher levels. In the United States, national-level environmental goals are not, at this point, as formal and integrated as those in the Netherlands. Also, individual sectors in the United States have not adopted and tailored goals for their own environmental management efforts, as the various economic sectors in the Netherlands have done. An overall set of refinery-sector goals obviously would facilitate NPA goal setting at the refinery level. Without such established goals, the NPA Council must develop the goals on the basis of its own analyses of local conditions and, to the extent available, state- and national-level priorities and goals. 


\subsection{Establish the Revised NPA Baseline}

Once the NPA Council establishes the goals, it oversees the development of a refineryspecific baseline that includes residual releases and costs of environmental management. This baseline provides a benchmark for setting limits and against which future releases can be compared. Workshop participants sought clarification on several issues regarding the NPA baseline. These include the following: whether the public would be involved in setting the baseline; whether the baseline would reflect current operating conditions or current regulations; how refineries would be prevented from setting the baseline lower than it actually is to imply greater progress; how to identify sources of releases and pollution prevention opportunities; whether all residuals, regardless of impact, would be treated the same way; how the data would be validated; and how costs would be used. As described below, the revised NPA baseline addresses these issues.

In establishing the revised NPA baseline, the NPA Council would oversee three activities:

1. Develop inventory of refinery residuals,

2. Develop inventory of environmental management costs, and

\section{Document results.}

The NPA Council would be responsible for identifying the data to be included in the baseline, how the data will be collected and verified, and how the data will be used. It will also oversee the process, which, because of its detailed nature, will be conducted by staff from the refinery with input and possible assistance from regulatory agencies and local citizens. Collecting the data will be a time- and resource-intensive undertaking. Time and resources could be reduced by focusing data collection on, for example, the most harmful pollutants or most prolific sources. Thus, in addition to the above three tasks, the NPA Council could attempt to narrow the undertaking by screening and prioritizing residuals and sources for data collection and limit setting. Such screening levels would be determined on the basis of factors such as refinery size and complexity, available resources, and location relative to residential areas, in conjunction with the overall goals established at the beginning of the process.

\subsubsection{Develop Inventory of Refinery Releases}

To determine the most effective means of improving environmental performance, a baseline of residuals generated by the refinery would be established through facilitywide observation, interviews, record reviews, measurements, and, if necessary, estimation. This baseline would identify each residual (i.e., any solid, liquid, or gas in any medium or phase that is not an intermediate or end product for sale or legitimate use in commerce) before its recovery, recycling, treatment, or disposal. Ideally, the baseline would identify the quantities of all residual releases from all sources at the refinery, whether or not the releases and sources were currently regulated. The identification would be done because many residuals and sources may not be regulated but may still contribute significantly to a refinery's total emissions. However, as noted, resource constraints may require the NPA Council to limit the number of residuals or sources 
included in the baseline. All identified residuals would be linked to a process or source and a medium to facilitate the identification of pollution prevention opportunities.

The inventory development process will involve the refinery, the regulators, and the public, via the NPA Council. As was the case for the revised RBB, the process for identifying releases could be modeled on the Yorktown study (see Section 5.4.1); the lessons learned from that approach could be incorporated with stakeholder input. For example, local citizens might be able to identify sources of fugitive emissions near plant boundaries that are not currently regulated. They might also be able to provide the NPA Council with data on local health impacts that could help focus residual and source identification efforts. It is conceivable that some citizens (e.g., retired refinery workers) might wish to work with refinery staff to participate in measurement efforts. Such assistance, combined with oversight from the regulatory agencies, would help assure the pubic that the collected data were valid and that the refinery was establishing a baseline consistent with actual data.

Some workshop participants asked whether the baseline would reflect current operating conditions or current regulations. Because the release limits will be negotiated independently for each refinery, and because actual releases will be compared with baseline releases for that refinery to determine if goals are being met, the baseline should reflect current operating conditions. Current and anticipated regulatory requirements might provide guidance in setting the limits. However, goals will be set in terms of quantities of releases or percent reductions from the baseline. The NPA Council may consider recent capital additions and resulting residual reductions that may impact the baseline when setting the limits.

As noted in Section 5.4.1, identifying all sources and all releases is a resource-intensive undertaking. However, the NJDEP has suggested a means for locating where hazardous substances are used or generated within a facility; the NPA Council could use this means as it develops the inventory (NJDEP 1999a). The suggested means is based on a materials balance approach (also known as throughput accounting or total use accounting) and is used by New Jersey facilities seeking facilitywide permits. The approach begins by listing the products (including intermediate products) generated at the facility. For each product, analysts use process flow diagrams to identify and describe processes, from end to beginning, and to identify sources. Components of the process flow diagrams include raw material inputs, products, and nonproduct output streams from various sources. (Sources in this context are locations in a production process where nonproduct output leaves a production process prior to treatment. This interpretation differs from the conventional definition of source as a place where a permitted release leaves a facility and enters the environment.) Analysts also conduct a qualitative materials accounting check to ensure that no nonproduct output streams have been overlooked, by determining where process inputs appear in product, pass through a source as nonproduct output, or are consumed. Acknowledging that process flow diagrams will not capture fugitive emissions, the approach suggests that analysts conduct a facility walkthrough to follow each process from the point where hazardous substances first enter the facility to where product and nonproduct outputs are generated and moved off site. Areas where nonproduct output leaves a process in an unusual way (e.g., through open vessels, leaks) are identified and added to the relevant diagrams. Processes that do not appear on the diagrams, such as cleaning equipment with a hazardous substance, should be considered processes that create a "desired result" rather 
than a product. This residuals identification process provides a tested approach that can be used by the NPA Council to begin conducting a residuals baseline inventory, and the NPA Council can modify the approach as appropriate.

\subsubsection{Develop Inventory of Environmental Management Costs}

Benefits to the refinery from implementing the NPA (or any other regulatory improvement) pertain principally to costs. While enhanced public recognition, improved community relations, and other nontangibles can be positive derivatives from using an alternative regulatory approach, the chief benefit is better cost control or even cost reduction. Although difficult to quantify, understanding the current or baseline costs of environmental management will help the refinery identify and assess the benefits of implementing an NPA. Unfortunately, as environmental cost accounting literature reports, many environmental management costs are misallocated (generally to overhead) and therefore cannot be tracked, evaluated, or easily reduced. For example, the Yorktown refinery study found that prior to investigation, environmental costs were estimated to be $3 \%$ of noncrude operating expenses, but the study showed these costs to be at least $22 \%$ of noncrude operating costs (EPA 1997a). Identifying and allocating environmental management costs at the outset will enable the refinery to measure the cost impacts of implementing the NPA over time. It will also make it easer to target new technology use or pollution prevention options on the basis of potential cost savings, therefore contributing to overall cost-effective operations.

Accurately identifying and allocating environmental management costs is not a trivial exercise. An early evaluation of the Dutch NEPP showed that at least half of the companies surveyed were unable to estimate the cost of environmental measures, largely because the environmental measures are process-oriented and difficult to discern (KPMG Milieu 1993). According to the American Petroleum Institute's (API's) Pollution Prevention Task Force, while industry engineering personnel are proficient at identifying costs to implement new projects,

their skills in identifying the benefits of pollution prevention may not be as well developed. Also, the complexity of refining operations can cause analysts to overlook some waste-related costs. Ignoring such costs prevents facilities from evaluating the "true" costs of waste generation and environmental management, which, in turn, constrains companies from accurately evaluating potential pollution prevention projects (API 1993). Before conducting an inventory of environmental management costs, the NPA Council will need to determine the scope and scale of the data collection effort and the potential uses for the data collected. Scope refers to the types of costs to be inventoried and could include conventional costs (e.g. capital equipment, labor), potentially hidden costs (e.g., reporting, inspections, protective equipment, environmental insurance), contingent costs (e.g., penalties, property damage, legal expenses), or relationship/image costs (e.g., relationships with lenders, relationships with investors). Scale refers to the level at which costs are inventoried (e.g., plant level, process level.) Baseline cost data can be use to compare costs over time for informational purposes, facilitate budget decisions, and make product pricing decisions. The NPA Council is urged to review the current 
literature on environmental cost accounting as it develops the baseline cost inventory. The EPA has prepared several helpful environmental cost accounting reports. ${ }^{9}$

Once the NPA Council has determined the scope, scale, and purposes of the cost inventory, it should agree on the approach for the data collection activities and oversee them. A generally accepted approach would include the following steps (EPA 1997b):

- Identify environmental management costs,

- Prioritize and select costs for further investigation,

- Quantify or qualify the costs, and

- Allocate the costs to responsible processes or products.

Additional detail and factors to consider when undertaking this process are discussed in EPA (1997b).

When conducting the inventory, the NPA Council should consider total costs. The life-cycle costing concept uses different terms to capture total costs (e.g., direct, indirect, recurring, nonrecurring), but the important issue is that the NPA Council agree on the cost data it plans to collect, the level of detail at which those costs will be collected, and how the collected cost data will be used.

\subsubsection{Document Results}

The results of the baseline inventory of residuals and costs should be documented in a clear and transparent form for use by the NPA Council. All baseline residuals data should be available to the public. (Many of these data are already publicly available via the TRI.) Because some of the cost data may be sensitive, the NPA Council should determine, on the basis of information supplied by the refinery, balanced with an assessment of the public's need to know, the extent to which cost data should be made public.

Some workshop participants asked how the NPA would treat confidential information. The NJDEP has developed a confidentiality policy that may provide guidance for the NPA Council as it addresses this issue. In 1993, the NJDEP established a task force to address confidentiality issues. Its recommendations guided the NJDEP in developing procedures to protect confidentiality needs of businesses. The current policy requires that information in a facility's pollution prevention plan be treated as confidential and remain at the facility unless and until the NJDEP requires submittal of the plan for review. The NJDEP has the authority to inspect the plan on site, but New Jersey State law restricts the inspectors from disclosing data on trade

9 For example, see An Introduction to Environmental Accounting (EPA 1995b), Environmental Cost Accounting for Chemical and Oil Companies (EPA 1997a), Applying Environmental Accounting to Electroplating Operations: An In-Depth Analysis (EPA 1997b), and the EPA's Environmental Accounting Web site. 
secrets in the plan and subjects any inspector who discloses such information to dismissal, suspension, fines, or conviction. The NJDEP is legally entitled to postpone a confidentiality determination until it receives a request for disclosure from a person not authorized to see it. The NJDEP reports that only seven of 600 facilities have submitted confidentiality claims regarding throughput data reporting requirements, and no specific issues of concern have arisen (NJDEP 1999d).

\subsubsection{Prioritize Pollutants for Setting Release Limits}

Conducting residual and cost inventories can be time and resource intensive. Also, most stakeholders agree that some residuals may pose potentially greater harm to public health and the environment than others. Finally, the effort to negotiate limits for all residuals to all media simultaneously may be so great that participants may become too overwhelmed to conduct the baseline and subsequent tasks, thereby forgoing the intended benefits of regulatory flexibility and improved environmental conditions. Many workshop participants asked if the residuals would be weighed or if they would all be treated in the same fashion. The revised NPA does not require all residuals to be treated in the same manner. To set priorities on the residuals identified in the baseline, the NPA Council should first review the goals it set at the outset. These goals may readily dictate the priorities. For example, the goals may have been to reduce releases of greenhouse gases (based on a national goal) and reduce toxics (based on a local concern of residents.) The NPA Council could then propose setting limits for residuals that contribute to meeting these goals first. The NPA Council may also develop a screening or ranking process to prioritize the residuals before attempting to set release limits by using such factors as the amount and source of specific constituents and the medium into which they are discharged, human health effects data, and interactions with other constituents. For many contaminants (e.g., criteria air pollutants), considerable data are available through the EPA and the literature. For others (e.g., toxic metal compounds), data may be limited, but as environmental research continues to expand, additional information is expected to become available that will facilitate such scaling.

While the baseline provides a benchmark for setting limits, the NPA is not limited to the residuals identified in the baseline. For example, the NPA may also include specific pollution prevention or new technology implementation requirements. Thus, the baseline may also entail a pollution prevention assessment, which identifies potential areas for emissions reduction through pollution prevention activities.

\subsection{Set Risk-Based Release Limits in the Revised NPA}

Key distinguishing features of the NPA, when compared with the current regulatory system and many other reinvention approaches, are the negotiations and the resulting agreement. Although other regulatory reinvention approaches use negotiations, facilitywide or multimedia permits, and covenants, the revised NPA combines all of these components and provides a long enough timeframe to allow refineries to implement process changes or other approaches to meet the residual release limits set in the NPA. 


\subsubsection{Background on Negotiations, Covenants, and Permits}

The following sections provide background and examples of how negotiated rulemakings; European covenants; technology compacts; Japanese environmental agreements; and multimedia, facilitywide, integrated permits have been used by various agencies and organizations and how they can be applied in the NPA.

\subsubsection{Negotiated Rulemakings}

The Administrative Procedures Act (APA) of 1982 (United States Code, Volume 5, pages 551-559 [5 USC 551-559]) established procedures for agencies to follow in developing regulations. The APA requires an agency to notify the public that a rule is being considered and allows interested parties to submit data, comments, or arguments regarding the potential rule. However, the normal rulemaking process can become adversarial, with such submissions made primarily to provide documentation that ultimately can be used in legal proceedings. Parties often take extreme positions, withhold information, and hide potential areas of compromise (Woods 1995). In an attempt to halt this trend, and to develop more acceptable rules and minimize subsequent legal challenges, the Administrative Conference of the United States recommended allowing affected parties to negotiate with each other to develop proposed rules. In 1990, Congress passed the Negotiated Rulemaking Act (Public Law 101-648), which established a framework for and encouraged the use of the negotiated rulemaking process. The process generally includes the following components:

- Formal commitment from the parties to participate fully and in good faith and to communicate with their constituents who are not a part of the negotiating process;

- Training sessions with a mediator to enhance negotiating skills and practice consensusbuilding techniques;

- Setting of ground rules (e.g., schedules, confidentiality protections, rights of withdrawals, standards of communication with outside parties); and

- Keeping all members informed and providing for input, and allowing small groups to work on specific issues.

Over the past several years, the EPA and other federal and state agencies have used regulatory negotiations successfully (EPA 1998d). The Better Business Bureau has identified several advantages and disadvantages of regulatory negotiation (Woods 1995). Advantages include the following:

- Agencies can receive more complete information than they could if they relied on their own expertise or data submitted solely to support affected parties' positions.

- Affected parties are more likely to communicate their true interests rather than merely stating their positions. 
- Because collaborative efforts are used to solve problems, solutions tend to be more creative than those developed under traditional rulemaking processes.

- Resulting rules are more likely to be accepted and enforced because they are perceived as having greater legitimacy than those developed solely by regulators.

- The agency can conserve resources used to develop and enforce its rules.

Disadvantages include the following:

- Initially, the regulatory agency and the affected parties spend more resources, and some parties may be unable to participate without outside support.

- The approach can appear to focus on private rather than public interests.

- Some critics charge that rules resulting from regulatory negotiations are formed on the basis of compromise rather than empirical data.

The successes of the regulatory negotiation approach to date, combined with the ability to address some of its criticisms, suggests that it is an appropriate mechanism to use in the revised NPA. Thus, the negotiation process in the revised NPA will include (via the NPA Council, which will be the negotiating body) representatives from all significantly affected parties. Because all parties are represented, it is expected that empirical data will be important in justifying claims and positions. Although resource issues will have to be addressed, it seems appropriate to dedicate resources to negotiations early in the process, given that the benefits of the NPA process are expected to be significant over the longer term.

\subsubsection{European Covenants}

To date, other countries have used covenants (agreements between industry and the regulator or among industry, the regulator, and the public) more than the United States has. The following discussion is derived from a 1997 paper that describes the use of environmental agreements in Europe (Rehbinder 1997). Europe has used two types of environmental agreements. The first, environmental contracts or covenants (such as those used in the Netherlands), legally bind the parties. The second, nonbinding contracts or "gentlemen's agreements," are those in which industry, after negotiating with the State, issues a formal declaration or "self commitment." Such self commitments are common in Germany. Austria, Denmark, and Sweden use both types of agreements. Existing environmental agreements cover products (e.g., packaging, labeling), product wastes (e.g., recycling, end-of-life vehicles), and emissions reduction. Most contain reporting and monitoring provisions to ensure that the agreement is effective and to gain sufficient public acceptance.

- The Netherlands experience with covenants. In the past 20 years, more than 100 environmental covenants have been signed in the Netherlands. The basic metals covenant is similar to the agreement envisioned for the revised NPA, except that the metals covenant is at the sectoral level while the revised NPA is at the refinery level. Parties to 
the agreement are the primary metals industry association, major producers, and various regulatory authorities. Although the covenant is binding, it does not substitute for applicable legal provisions; rather, it provides terms for permit authorities to consider when issuing or modifying permits. The covenant addresses all major air, water, soil, and waste, emissions, radiation, noise, odors and industrial safety. It establishes interim and final reduction targets from a 1985 base year, and the parties commit to achieve all targets. The agreement also provides for translating the targets into requirements for individual firms through CEPs, which are negotiated between individual firms and the regulatory authority. A steering committee composed of representatives from the Primary Metals Foundation and the participating ministries ensures that the sum of the reductions provided by the individual CEPs meets the sector-level targets. Although the public is not a direct party to the negotiations, drafts of the CEPs are subject to public participation. If the regulatory authorities concur with the CEP, they agree to consider it when granting or modifying permits. This generally means that the CEP will be binding; if the plan is violated, existing regulations are applied. Companies must submit annual reports that describe progress made in achieving the company targets to the regulatory authorities, but there is no independent verification mechanism. The steering committee may adjust the plan, typically by prolonging deadlines, if there are enough economic or technological problems to justify prolonging the deadlines or if newly acquired knowledge or emissions profiles suggests that adjustments are appropriate. All parties to the covenant must agree to any such adjustments.

- The German experience with self-commitments. German industry has issued about 60 environmental self-commitments during the past 20 years. These commitments are unilateral declarations of national associations (or in some cases individual firms) that result from intense discussions with appropriate ministries. An example is the 1995 declaration of German industry to reduce $\mathrm{CO}_{2}$ emissions or energy production per production unit by $20 \%$ by the year 2005 , with a base year of 1987 . (After reunification, the base year was changed to 1990 to limit the crediting of "windfall profits" in emissions reductions from plant closures after reunification.) The declaration includes a complex monitoring system supervised by an independent economic research institute, and the results are publicly available. Nearly 20 individual self commitments from various sectors exist under this umbrella agreement, and many contain targets that exceed the general $20 \%$ target.

Some observers view the increased use of these types of commitments as an opportunity to implement flexible environmental policy, and others see it as a sign of regulatory weakness. While industry and many regulatory authorities believe environmental agreements lead to efficiency gains, flexibility, and reduced operating costs, they are less convinced that such agreements lead to increased development and use of new technologies. Opponents to environmental agreements (typically environmental groups and some regulatory authorities) cite poor industry compliance, the limited power of sector associations over firms, and negative public perception. Because environmental associations view the negotiation process as a means to weaken or postpone regulation, they want to limit the contractual approach to supplementing rather than substituting for existing regulations. 


\subsubsection{Technology Compacts}

Given the uncertainty about the impact of environmental agreements on the development and use of new technology, covenants in the form of technology compacts should be mentioned. Technology compacts would replace end-of-pipe technology requirements with process redesigns based on phased increments of technological change developed by users of the technology. Such technology compacts would create long-term processes for improving technology. While no examples of existing technology compacts could be identified, researchers have suggested the use of such compacts to increase commercialization of new technologies (Banks and Heaton 1995). Such technology compacts would provide long-term goals for technological change directed toward enhanced environmental performance and improved market competitiveness. Parties would include relevant regulatory agencies and individual firms or groups of firms. Other parties could include labor, customers, and technology developers. In a technology compact, a facility (or firm) would commit to performance goals that produce environmental benefits significantly greater than current standards over a 10-year period. Regulators would commit to enforcement flexibility, acceptance of new technology, and consistency in regulatory requirements. Compliance evaluation would include a mid-course assessment of progress and problems.

\subsubsection{Japanese Environmental Agreements}

In Japan, roughly 40,000 environmental protection agreements have been implemented since 1964 (Enterprise for the Environment 1996). Parties to these agreements are typically local governments and individual companies, with public interest groups or local citizens participating in the negotiating process and in the final agreement. Most agreements address methods of reducing emissions for a specific company and provide for emissions limits, monitoring, and inspection, and compensation for damages in the event of a violation. Often, the agreements will include pollutants or actions that are not statutorily regulated. Both local governments and local citizens participate in the inspections. Violations can result in large compensation awards, and most agreements stipulate the placement of funds on reserve or the purchase of insurance for compensation purposes. The agreements are popular in Japan because they provide flexibility and can be tailored to a specific industry, community, and set of citizen concerns. Problems cited with the agreements are that vague provisions are difficult to enforce, that industry tends to have more bargaining power than the local government, and that citizen participation is only offered to some members of the community.

\subsubsection{Multimedia, Facilitywide, Integrated Permits}

Permits that cover multiple environmental issues at a single facility are generally labeled multimedia, facilitywide, or integrated, or some combination of these terms. Advantages of such comprehensive permits include the following:

- Elimination or reduction of conflicting or duplicative requirements;

- Reduction in transfers of pollutants across environmental media (particularly if the permitting body includes representatives from the various media); 
- Increased flexibility to meet requirements cost effectively;

- Stimulation of experimentation with new technologies and process designs; and

- Increased certainty and more stable operating environments.

Two examples of such permits follow here.

- Integrated permitting in Sweden. Sweden's permitting system, which regulates total emissions to air, water, and land on a facility-specific basis, embodies two key concepts. First, pollution control is accomplished by using the most advanced and efficient technologies practicable. Second, standards apply on a case-specific basis. Thus, the degree of control of a given pollutant can vary with the site. The following highlights of a Saab-Scania integrated permit (Hinrichsen 1990) provide information relevant to the revised NPA. The first part of the three-part permit contains detailed production information supplied by the company (including data on raw material consumption and waste streams), a detailed list of discharges to the environment, and the company's proposal for future emissions levels. The second part is an analysis of the proposal by the local and national environmental regulatory agencies. The third part documents the releases allowed to various media. Existing pollution control technologies and the general state of technological achievement are used to determine release levels. The entire permit is roughly 50 pages long, and a separate document provides a list of parameters to be monitored, monitoring methodologies, and reporting requirements. In some cases, measurements for priority pollutants are to be taken every minute, and if discharges rise above permitted levels, plant managers can make immediate adjustments. The company is subject to compulsory inspections of the plant's monitoring techniques and reporting procedures. Violations or negligence are penalized by fines, imprisonment, or fees that correspond to the amount of money the company made by avoiding compliance. Issues of concern related to the permitting process include the following: vague wording can thwart efforts to reduce emissions and adequately monitor and inspect operations; legal sanctions may not be sufficient; and the lack of a national policy may hinder efforts to develop environmental priorities.

- State-specific, facilitywide, multimedia permits. Section 6.1 described multimedia permitting programs in New Jersey, Oregon, and Texas. These programs appear to be successful, but none has existed long enough to allow for a meaningful, comprehensive evaluation. However, the failure to implement a facilitywide permit for a $3 \mathrm{M}$ plant in Minnesota, despite a two-year effort to do so, does provide some instruction to be applied to the development of an NPA. In 1994, 3M and the Minnesota Pollution Control Agency (MPCA) began designing a facilitywide multimedia permit under Project XL. The permit would have set requirements typically found in multiple permits covering air, water, and hazardous wastes. It was to provide flexibility to the plant, allowing it to make operational changes as long as plantwide emissions limits were not exceeded. Features of the permit included the following: 
- Replacement of 22 individual permits with one permit;

- Inclusion of air, water, and solid waste requirements;

- Verification of facilitywide emissions limits by using material inputs and material contents of final products;

- Innovative environmental management system to integrate the environmental protection function into the overall strategic operations of the company;

- Stakeholder involvement (the state regulatory authority would have the authority to revoke the permit if $3 \mathrm{M}$ failed to satisfactorily address substantive issues raised by a majority of members of its stakeholder groups);

- Reporting of actual emissions on the Internet; and

- Monitoring and evaluation procedures that would have relied on public disclosure of mass balance input-output statistics.

After two years of hard work by all of the parties involved, negotiations broke down, and the permit was never issued. An analysis of the problems encountered during the negotiations suggested several reasons that the permit was not granted (Marcus et al. 1999). They included the following: conflicting goals, insufficient legal means, insufficient delegation of power from upper levels within EPA, lack of coordination within different organizations, insufficient trust, and the inability to anticipate and address barriers. The $3 \mathrm{M}$ permit and the NPA contain many similar elements. The NPA Council can benefit from the lessons learned in the $3 \mathrm{M}$ case by stressing the need for consistent and agreed-upon goals and the need for commitment by all parties to reach an acceptable agreement.

\subsubsection{Factors to Consider}

On the basis of comments received from workshop participants, a review of potential benefits and pitfalls of various negotiating instruments and agreements, and lessons learned from the above examples for negotiated agreements, the project team suggests the NPA Council consider the following factors when setting the limits and negotiating the agreement.

\subsubsection{Public Participation}

Obtaining input from all potentially affected parties at the beginning and throughout the process is critical. The NPA Council, which consists of both nonvoting and voting representatives of the affected community, will ensure not only that such input is received, but also that it is used in the actual goal setting. 


\subsubsection{Process for Setting Limits}

Factors that the NPA Council should consider in setting the limits include the following: the goals that the NPA Council set before establishing the baseline, baseline emissions, current regulatory requirements, anticipated regulatory requirements, and recent investments the refinery may have made to reduce emissions. If not already included as part of the goals, other factors to be considered include the relative harm and contribution of specific contaminants to the health and environment of the local community, the use of pollution prevention techniques to reduce releases, and current and expected technological changes that could facilitate emissions reductions at the refinery. Negotiated release limits will be, at a minimum, as demanding as those required by current aggregate regulations.

The negotiated agreement may need to address refinery production issues. For example, volumes and types of products may change during the performance period. The NPA Council should discuss whether and how such anticipated (or unanticipated) changes would impact the release limits and targets. For example, while a premise of the NPA holds that the refinery can make process or product changes as long as the limits are not exceeded, a major refinery expansion may require an upward adjustment in the limits. The State of New Jersey addresses this issue by requiring limits to be expressed on a per pound of product basis.

The NPA Council may also decide to use the results of the pollution prevention assessment (if conducted while the baseline was established) as a basis for setting limits or requiring certain pollution prevention activities as part of the permit. In setting the limits, the NPA Council will need to consider realistic time estimates for new technology implementation. The NPA Council may also consider setting priorities for the limits. Such priorities will help the refinery establish optimal approaches, and the NPA Council could use these priorities if it needed to assess penalties. Thus, the penalties could be scaled to increase as the priority of the goal increases. All limits are envisioned to be media- and pollutant-specific rather than source- or process-specific.

\subsubsection{Unit of Measure for Limits}

The nature of the pollutant will dictate the form (or units) of the limit. For example, for criteria air pollutants and greenhouse gas emissions, the form should probably be in terms of a percentage reduction and a total pound-per-year reduction from baseline emissions. For toxic air pollutants, the form should reflect the fact that short-term increases can pose hazards to the local population that might be masked by annual measurements. Thus, the limit for toxic pollutants might be expressed in terms of pounds per day or pounds per hour. As noted, limits will be set on a facilitywide basis, so that the refinery can alter processes within the refinery, as long as the total refinerywide caps are not exceeded. Although some existing facilitywide permit systems (e.g., New Jersey) base limits on materials use and set limits both at the facility level and at the process level, the generic NPA assumes limits will be set on releases from the refinery as a whole. This facilitywide aspect is similar to the bubble concept in the RBB, except that the RBB considers risk as the unit of currency, thereby allowing trades across media and pollutants. The NPA does not provide for trading. 
The agreement may also include performance measures that consider information beyond satisfaction of emissions limits or reduction goals. Additional performance measures could include introduction of new process or control technologies, incorporation of stakeholder views into decision making, improved time and effort associated with compliance assurance, and cost savings to the refinery and the regulators (see Section 5.6.4).

\subsubsection{Performance Period}

As noted, release limits will form the basis of the facilitywide permit or NPA. The length of the permit will be negotiated. However, after examining the lengths of similar permits under other programs, and in acknowledgment of the fact that implementing process changes can require significant planning, design, and financial commitments, the project team recommends a permit length of 10 to 15 years or more. Such a period allows for the achievement of long-term environmental improvements through effective and efficient process and design changes. The Dutch NEPP, for example, allows for significant change to occur over a 25-year period (i.e., within a generation). To allow for long-term process gains that cannot be measured on a year-toyear basis, and also to allow for opportunities to review progress and address potential shortcomings of the NPA over time, interim milestones or checkpoints are recommended every four or five years. These milestones will provide an opportunity to realign goals or dates if necessary. For example, if an expected new technology was actually not available when anticipated, the NPA Council could adjust the performance period of the agreement. The NPA Council might establish pollutant-specific milestones if deemed appropriate. In addition to these structured opportunities for progress review, the NPA Council might require the refinery to make information on emissions reductions goals and actual releases available for public review via the Internet or other reporting format. Experience indicates that such public reporting (e.g., as required by the TRI) provides powerful incentives to continually reduce releases.

\subsubsection{Format and Content of Agreement}

The agreement will focus on limits, schedules, monitoring, reporting requirements, and penalties. In addition to providing pollutant- and media-specific limits, the NPA Council may also decide it should require other conditions (e.g., the implementation of certain pollution prevention measures). Similarly, depending on the individual situation, the permit might address resource use, community involvement, or other factors consistent with the overall goals of environmental responsibility, economic performance, and pollution prevention technology development and use. The NPA should also allow for modifications on the basis of milestone progress. The NPA Council would need to thoroughly review the grounds for any proposed modifications. It would need to balance the objectives of providing the refinery with long-term assurance that regulations and requirements will not change with those of demonstrating that the environmental goals of the agreement are being met. The permit would also contain procedures for resolving disputes. Consistent with the program's overall goal of providing flexibility, the permit would contain no specific end-of-pipe or other technological requirements. As a result, the document is expected to be shorter than traditional permits. On the basis of existing permit programs, the agreement could be as few as 10 pages and will probably be no more than 50 pages. To ensure transparency, the permit should include tools to enhance understanding, such as a glossary of terms, a user's guide, and possibly a "plain English" version. 


\subsubsection{Information Dissemination}

The negotiated limits for the refinery will be publicly available via the Internet. The NPA Council should also consider posting the entire NPA on the Internet. Doing so will help address the need for public access to information and will help the public evaluate subsequent reports to assess progress.

\subsubsection{Dispute Resolution}

While the NPA is based on negotiations, disagreements may arise during those negotiations and during the NPA's implementation. Various dispute resolution mechanisms could be used, but the project team believes that the time, cost, and ill will that often accompany formal legal procedures should be avoided by using court adjudication only as a last resort. Disputes should be resolved using a continuum of alternative dispute resolution (ADR) techniques, beginning with the most basic (dialogue between the disputing parties) and proceeding, as necessary, to binding arbitration. The following approaches, in order of implementation, are recommended:

- Dialogue. When a dispute occurs or is perceived to have occurred, the disputing parties should discuss and attempt to resolve the concerns among themselves.

- Facilitation or assisted negotiation. If such bilateral discussions fail, the parties can select a third party to help them articulate their objectives, set a schedule for completing talks, and identify and overcome obstacles to resolving the dispute. If the dispute is technical, a third party with expertise in the subject matter could help investigate and determine findings of fact.

- Mediation. If facilitated negotiations fail, the parties could select a neutral third party, with no decision-making authority, to help them reach a voluntary, negotiated settlement. Mediators may be more active than facilitators in identifying and overcoming barriers to resolution.

- Arbitration. If mediation fails, the parties could move to arbitration, a more adversarial proceeding where a neutral third party or panel hears the dispute and renders a decision. Arbitrators generally have greater substantive knowledge of the technical issues rather than the legal issues. Arbitration may be binding or nonbinding.

The EPA's Office of Enforcement and Compliance Assurance advocates the use of ADR at any point where negotiations are possible. It suggests several advantages of using the ADR process (EPA 1995c). Those pertinent to the revised NPA include the following:

- Obstacles to agreement can be identified quickly.

- Dialogue can be restored when communication has broken down.

- Mediators can help prevent stalemates by narrowing the issues and identifying obstacles to resolution. 
- Constructive working relationships can develop.

- Parties can be encouraged to identify settlement options tailored to their particular needs.

- Ongoing relationships can be preserved.

- Transaction costs can be lower.

\subsection{Assure Compliance in the Revised NPA}

The revised NPA requires a greater degree of interaction among all interested parties to assure compliance than do traditional permitting approaches. Via the NPA Council, the public participates in the development of compliance assurance mechanisms, which include incentives for enhanced performance and penalties for violating limits or monitoring, reporting, or other requirements. The NPA is an enforceable agreement, and the public may play an active role in implementing compliance assurance mechanisms, such as reviewing reports and assisting with monitoring and inspection activities. The NPA strives to focus a refinery's resources on costeffective pollution prevention options and monitoring of progress. By allowing a refinery to develop and use innovative technologies, redesign processes, change catalysts, implement pollution prevention, and take other actions to satisfy the conditions of the negotiated agreement, the revised NPA provides maximum flexibility to the refinery. The following subsections describe various compliance measures envisioned as part of the revised NPA.

\subsubsection{Monitoring}

As noted in the revised RBB discussion (see Section 5.6.2), measuring performance should be based on release data collected by monitors as opposed to data derived from models of releases. Properly functioning monitors provide greater accuracy than models, and stakeholders generally have more confidence in data from monitors than from models. In developing monitoring requirements, the NPA council should consider how the results will be used. Monitoring can be done to accomplish the following:

- Determine any needs for real-time feed or process adjustments to avoid (or correct for) exceeding allowable limits;

- Provide direct input for publicly available reports;

- Evaluate progress against milestones; and

- Obtain process results, which might encourage a refinery to change processes to improve results. 
The NPA Council might require monitoring for other purposes as well. Once the purposes of the monitoring are identified, the NPA Council can determine what will be monitored and how frequently. It may also suggest methods for monitoring, but generally these methods will be determined by the refinery, as long as they produce accurate and timely results. The refinery will be responsible for implementing quality assurance protocols to meet the requirements of the revised NPA.

\subsubsection{Reporting}

The NPA strawman approach advocated streamlined reporting, and many stakeholders expressed concern that streamlined reporting would mean reduced reporting. They stressed that increased flexibility must also mean increased accountability, and such accountability is achieved largely through reporting. The revised NPA does not envision less reporting; rather it seeks more efficient, effective, and transparent reporting. Reporting in the revised NPA, as in the revised $\mathrm{RBB}$, reflects the understanding that not only is public reporting of clear, well-defined, and easily understood information critical to the success of a negotiated agreement, but publicly available information is expected to provide a powerful compliance incentive. Thus, reporting in the revised NPA should include not only raw data, but also the context of the data and annotations or explanations useful for interpreting that data. An example of such a report is the State of New Jersey's Internet-based report on toxic air pollution in the state. The report provides information not only on levels of air toxics but also on estimated risks of exposure to air toxics, suggestions on what residents can do to reduce air toxics, data on trends in toxic levels over time, and maps illustrating differences in concentrations across the state (NJDEP 1999b).

NPA reports should indicate the progress of the refinery in meeting its goals. The facilitywide permit program in New Jersey requires reporting on the progress of facility-level data for each pollutant and each process. Although not required, the program suggests that facilities should also report financial progress by using a cost accounting framework to assess the economic impacts of implementing various options. The Pollution Prevention Alliance (PPA), a group of local and national environmental and other public interest groups organized by the Environmental Defense Fund, has recommended ways to help ensure accountable reporting of progress (PPA 1996). The project team recommends that the revised NPA incorporate these recommendations, which include the following:

- Reporting tools must emphasize information that helps identify pollution prevention opportunities.

- Reporting systems must be understandable to all interested parties.

- Reporting information must be readily available to workers and the public.

- Reporting requirements should be consolidated to eliminate redundancies and better match the way businesses make decisions.

The New Jersey system also addresses confidentiality of facility data. Thus a facility

manager may submit a confidentiality claim for a progress report when the manager believes that 
the disclosure of the information is likely to cause economic harm to the company. When the regulator receives a public request to see a progress report for which a confidentiality claim has been filed, it will assess the claim and determine whether it is justified according to previously established confidentiality provisions (NJDEP 1999c).

Many of the reports envisioned in the revised NPA are similar to those in the revised RBB. Section 5.6.1 describes various types of reports (e.g., pollution prevention and other refinery actions, monitoring information, public participation) that strive to deliver meaningful data without excessive burdens on the preparers or readers of that data. As does the revised RBB, the revised NPA envisions that hard copy reports will be available and electronic reports will be accessible via the Internet. Ideally, many of the Internet reports would be generated in real time on the basis of data input directly from monitors, thus streamlining the reporting requirements.

To reward good performance, the NPA Council may consider a tiered reporting structure that links reporting frequency to emissions releases. Such an approach is a component of the Merck Project XL, which requires Merck to cap emissions of certain pollutants below recent actual levels, ensuring reductions in $\mathrm{SO}_{2}$ and $\mathrm{NO}_{\mathrm{X}}$ emissions to protect visibility and reduce acid deposition. The project will also test an innovative three-tiered approach to monitoring as an incentive to minimize emissions. Each tier has specific monitoring, recordkeeping, and reporting requirements tied to the emissions released by the plant. Thus, if the plant emits $75 \%$ or less of the sitewide cap, it must comply with the Tier 1 requirements; if emissions increase to $90 \%$ of the cap, more frequent and comprehensive Tier 2 requirements apply; and if emissions reach the cap, the most stringent Tier 3 requirements apply (EPA 1997c). Another option for the NPA Council to consider is self-certification. Self-certification allows facilities to certify compliance with limits without filing detailed reports, while ensuring regulatory and public access to data when needed. For example, one of the goals of the Weyerhaeuser XL project is to reduce the burden of unnecessary paperwork while simultaneously enhancing stakeholders' abilities to understand the environmental benefits of the project and track the facility's compliance with regulatory requirements and progress toward goals. Thus, the Project XL agreement calls for Weyerhaeuser to submit annual and mid-year summary reports to stakeholder groups and regulatory agencies, and to make available all backup data and reports to stakeholders on request (EPA 1997d).

\subsubsection{Inspections}

Because the NPA (similar to the RBB) does not require the refinery to use specific technologies or approaches to meet goals, rigorous site inspections are not needed to determine if such technologies are being used properly. However, the NPA Council should provide for inspections of monitoring equipment. These site inspections should not burden the refinery or those conducting the inspections but should complement the reporting mechanisms by ensuring that monitoring technologies are performing as designed and measuring the correct pollution parameters at the right time. The NPA Council may consider having local citizens participate in such inspections to provide meaningful stakeholder input with regard to compliance assurance and to reduce the burden on regulatory agencies. 


\subsubsection{Incentives}

The negotiation approach itself is an incentive in that it allows a refinery to work "outside the box" of traditional command-and-control system requirements. Nonetheless, the NPA Council could provide additional incentives to encourage a refinery to meet performance goals ahead of schedule or to surpass the performance goals specified in the NPA. For example, if the refinery met its goals significantly ahead of schedule (the exact amount would be set forth in the NPA) or if it significantly surpassed its milestone goals, it could bank a portion of such credits against future required emissions reductions, use them to negotiate limits applicable to future refinery expansions, or perhaps negotiate a trade against another pollutant goal, which, for some unexpected reason, had been difficult to meet.

\subsubsection{Penalties}

The revised NPA, like the revised RBB, will specify actions for which penalties may be assessed and the nature of such penalties. These actions could include the following: exceeding the negotiated performance limits; failing to maintain proper functioning of monitoring equipment; failing to provide reports as scheduled; and providing inaccurate reports. The revised NPA envisions monetary and nonmonetary penalties that could be structured to increase with the degree or frequency of violation or with the the potential hazard of the pollutant involved. For example, a monetary penalty could scale the cost per ton of pollutant exceeding the limit according to the potential harm of the pollutant. Similarly, lower penalties could be assessed if a failed control measure used a new pollution prevention technology that simply did not perform to expectations, and the refinery agreed to replace or modify it so that the expectations were met.

In assessing penalties, the NPA Council should avoid creating cross-media transfers. Such transfers could result if a given quantity of a specific contaminant released to the air posed greater harm than the same quantity of the same pollutant released to water, and the cost to reduce the quantity released to air is greater than the cost to reduce the quantity released to water. The NPA Council should review such cases to avoid unintended shifts in releases to media that could result in greater harm and should consider adjusting penalties to account for such potential shifts. The NPA Council may decide to consider other factors in assessing penalties. For example, when assessing penalties for environmental violations in New Zealand, the courts consider not only the nature of the environment affected and the extent of damage caused, but also such factors as whether the violation was deliberate, the attitude of the violator, remorse, and extent of any profits realized by the violation (Gow 1995).

Regardless of the penalty structure, the NPA Council should ensure that penalties are significant enough to discourage noncompliance. The NPA Council may decide that severe violations (as defined in the NPA) would be penalized by reverting to the otherwise applicable regulations, perhaps with additional penalties.

\subsubsection{Compensating Local Interests}

The concept of directing penalties to communities that was set forth in the NPA strawman approach was revised to include nonmonetary contributions that accomplish the same community 
benefit. Examples of both kinds of contribution (monetary and nonmonetary) exist. For example, the TNRCC has approved several projects in which penalty dollars assessed for violations of water quality or solid waste regulations were directed to specific projects to benefit local communities. For example, the City of Harlingen, which was fined $\$ 41,000$ for water quality violations, will create wetlands and ponds on more than 250 acres to complement a wildlife and nature park project (BNA 1999c). Nonmonetary compensation could be modeled after the EPA's supplemental environmental projects (SEPs), which are environmentally beneficial projects that a violator agrees to undertake but that are not otherwise legally required. The EPA has suggested several categories of SEPs, including public health, pollution prevention, pollution reduction, environmental restoration and protection, promotion of environmental compliance, and emergency planning and preparedness. Examples of SEPs include (1) implementing a comprehensive asthma detection and treatment program for schools in a Pennsylvania county, valued at \$250,000; (2) funding a citywide lead poisoning prevention project, valued at $\$ 200,000$; and (3) donating a hazardous materials response and command vehicle to the city of Philadelphia and restoring 2 acres of tidal wetlands along the Schuylkill River, valued at $\$ 655,988$ (EPA 1999k).

\subsection{NPA Implementation Issues}

Workshop participants identified potential implementation issues associated with the NPA that centered around resource requirements, stakeholder roles, jurisdictional issues, and statutory conflicts. Many of these concerns can be addressed by applying lessons learned and arguments used in similar regulatory reinvention approaches that have addressed these issues. However, the potential statutory conflicts would need to be resolved before the revised NPA could be implemented. The following subsections identify potential implementation issues and provide suggestions for how they could be mitigated.

\subsubsection{Resource Requirements}

\subsubsection{Financial Resources}

All stakeholder groups expressed concern that implementing a new approach such as the NPA would require resources beyond those needed in the current system. Public participation entails time and financial commitments on the part of volunteers who may already be overcommitted to similar activities. Bringing all participants up to a minimum common denominator of knowledge may require training that will need to be funded or conducted by organizations with limited resources. Regulators noted that the costs to change internal procedures, reorganize offices, obtain knowledge about new monitoring technologies, and focus on multimedia enforcement approaches may be significant and that such costs can dampen state regulators' enthusiasm for accepting new approaches. Refiners also noted that although they favor increased flexibility, the resources they might need to redesign processes and acquire, test, and use new technologies could be so high that many of them would prefer to operate under the existing structure, where the requirements are, in many cases, known.

All of these concerns are legitimate. However, most of the costs will be incurred early in the process. The revised NPA is a long-term approach, and the cost savings are expected to result in 
the later years, as the redesigned approaches and new technologies result in lower emissions required by the negotiated limits, which are reductions from current or projected limits. As the environmental results begin to improve, it is expected that the regulatory costs will decrease. Inspections and reporting are expected to decrease as electronic monitoring of releases increases. At the same time, the increased use of negotiating techniques (which the EPA is promoting via regulatory reinvention approaches such as Project XL) will place all participants further along on the learning curve. The experience regulators gain as they use these approaches can be applied to the revised NPA.

Similarly, the public will become more educated and experienced as it participates in these activities. Knowledge will be easier to gain as more facilities and jurisdictions post environmental results and explanatory text on the Internet. Regarding refinery resources, the Dutch program may be instructive. Many Dutch companies have found that the process of developing a CEP "taps into new sources of creativity within the company, and environmental measures are often devised which turn out also to reduce costs. Environmental technology developed by a company can also often be sold to other companies with similar problems. The freedom to decide which environmental investments should be made and when also benefits companies financially" (Pronk and Blankert 1998). Regarding costs for specific investments, the Dutch have devised a method that uses internal indicators (profit margin, solvency margin, profitability, capitalization, quick ratio, and current ratio) and external indicators (competitiveness and market position) to analyze the impact of specific environmental costs in the context of a company's market position and economic prospects. The tool is being used to facilitate discussion about environmental investments for individual companies.

As experience with new approaches increases, the benefits gained from these approaches should not only translate directly into lower implementation costs but also encourage other new approaches, possibly creating a snowball effect that will, over time, reduce resource requirements. The increasing number and variety of pilot programs (e.g., Project XL, statesponsored facilitywide permitting programs) should provide real data to support or refute the validity of the above predictions. For example, the Dutch Covenants program is credited with reducing industrial emissions of $\mathrm{SO}_{2}$ by $44 \%, \mathrm{NO}_{\mathrm{x}}$ by $22 \%$, volatile organic compounds by $43 \%$, and fine particulates by $52 \%$ since 1990 . While not all sectors show the same level of progress, the Dutch note that environmental benefits should not be evaluated solely in terms of emissions reductions. Thus, the creation of a durable system through which government and industry have learned to work together is an important outcome of the new approach.

In the interim, the EPA could help fund alternative approaches such as the revised NPA in several ways. For example, it could provide grant money for testing an NPA approach. State regulatory bodies and the EPA could require that revenue from penalties for violations be placed in a fund to provide training and other assistance to help implement new programs. Also, the EPA could dedicate funding to research new technologies that individual companies may not be able to fund on their own. 


\subsubsection{Timing}

Workshop participants noted that the time required to address potential statutory issues; train regulators about multimedia, facilitywide approaches; and bring the appropriate parties together to establish a baseline, set limits, and negotiate an agreement would be significant. This concern is valid. Nonetheless, as are the financial resource requirements, the timing requirements are expected to be greatest in the early part of the process. The benefits of that up-front effort should become more evident in the middle to later periods of the agreement. The Dutch Environmental Ministry has concluded that the Dutch Covenants approach, including all of the discussion and negotiation, is a "fast-track way of initiating change" (Pronk and Blankert 1998). One Dutch environmental specialist compared the time to develop a typical covenant (about 3 years) with the time to prepare environmental permits in the United States (sometimes 8 to 10 years). To facilitate implementation, the refinery may consider dedicating one person to work on the NPA; in the Netherlands, a company environmental plan was completed in six months by one person hired specifically to develop the plan (Pronk and Blankert 1998).

One workshop participant (a local citizens group representative) suggested that the concern about timing is not about the speed of implementation. Rather it is about the fact that efforts to develop and pursue alternatives are often undertaken without being sufficiently followed up and implemented. Explaining that "we always start things, we lay the groundwork, we prepare the paperwork, and we listen to others, but we often do not pursue the next steps," this participant expressed concern that the process of developing the two alternative environmental regulatory approaches would stop before one or the other or both fully were developed and implemented. The representative emphasized the importance of having a vision and having the initiative to push it further in order to effectively change the current system.

Workshop participants suggested several measures to be undertaken in the near term to help facilitate eventual implementation. These included providing training for local citizens, expanding monitoring efforts by refineries, and making meaningful reports publicly available.

\subsubsection{Jurisdictional Issues}

The pollution control system in the United States can be characterized as a complex mix of responsibilities shared by different levels of government. For example, the EPA issues national standards for air and water pollution, but states implement these standards on the basis of permits generally negotiated and issued by state agencies. Some programs grant the EPA the authority to review permits; other programs do not. While state and local agencies generally conduct enforcement activities, the EPA and U.S. Department of Justice also have enforcement powers. State and local agencies are responsible for most pollution monitoring. Refineries and other industries have long decried this mix of players, noting that many of the permits they must obtain can conflict with, overlap, or duplicate other requirements. Changing the institutions so that a single, facilitywide, multimedia permit could effectively replace the existing mix is not a small challenge. However, other countries have similar situations and have worked to integrate activities. For example, before implementation of the NEPP, a Dutch company seeking an environmental permit had to work with a variety of autonomous federal, provincial, and municipal agencies as well as the local water board. Although these various organizations still 
exist, many of their functions have become integrated as a result of that country's new environmental policy. Thus, before a company can prepare its environmental plan (which implements the goals of the sector-specific covenant), all of the government agencies involved must meet to determine minimum environmental requirements. By so doing, the problems and potential solutions are viewed by all agencies simultaneously, thereby facilitating solutions acceptable and appropriate to all agencies.

\subsubsection{Role of National Environmental Groups}

Some state regulators expressed concern about the role of national environmental groups in the NPA, noting that often such groups can dissolve the consensus achieved by local parties. Regulators noted instances where a refinery, state regulators, and local citizens had agreed to innovative regulatory approaches with benefits for all. As an example, Texas regulators cited the Mobil refinery in Beaumont, Texas, located near a large minority community. Refinery personnel worked with community leaders and made progress with them. However, toward the end of the process, outside public interest groups became involved and raised issues that ultimately led to the demise of the project. Regulators expressed the view that national groups pressure local groups, threaten to sue the EPA, and use other means to sabotage agreements. Such examples can inject unease into the exploration and attempted implementation of alternative regulatory approaches. At the same time, they highlight the need for early involvement by all potentially affected parties. By airing all concerns early in the process, the chances of later failure can be reduced.

\subsubsection{Obtaining Buy-in for New Ideas}

As noted in Section 5.7.1, EPA, federal, and state environmental regulations reflect the single-medium laws from which they are derived. The focus of EPA, with offices dedicated to specific environmental media, may perpetuate a bias toward this structure that discourages consideration of multimedia approaches to environmental protection. As described in Section 5.7.2.1, these laws and their regulations define a wide variety of permits, schedules, enforcement, and standards. Concerns over these requirements discourage consideration of multimedia environmental programs. The threat of citizen suits also discourages regulatory personnel, who are responsible for enforcing a plethora of single-medium requirements, from experimenting with innovative approaches that deviate from established protocols.

EPA workshop participants noted that multimedia approaches are often not promoted because of insufficient information. This practice leads stakeholders to attack the EPA's science, undermining its credibility. Texas regulators said they were willing to consider innovative regulatory approaches but expressed frustration over the unwillingness of the EPA to embrace innovation. The project team recognizes that change is difficult. However, it again points to the recent undertakings in New Jersey, Texas, and Oregon regarding multimedia permits as well as a number of XL Projects that are implementing new approaches. These concrete examples, combined with an increasing recognition that the current system needs improvement, are expected to facilitate acceptance of new approaches over the next several years. 


\subsubsection{Federal Statutory Conflict}

As they will for the RBB, existing federal statutory and regulatory requirements will constrain implementation of the NPA. The NPA, like the RBB, uses a multimedia approach, whereas the current system uses a single-medium approach. Also, the revised NPA, like the $\mathrm{RBB}$, provides incentives to promote pollution prevention. Current laws provide few incentives and, in many cases, provide disincentives (e.g., requiring the use of particular technologies for particular emitting sources) for using pollution prevention technologies or reducing emissions from unregulated sources. Additional obstacles are created by the overlap and numerous inconsistencies among these laws (see Section 5.7.2.1). Even though the risk-related issues that are associated with the RBB are not associated with the NPA, most of the other potential statutory conflicts apply. Section 5.7.2.1 describes these potential conflicts and suggests potential approaches for mitigating them. 


\section{Section 7 Conclusions}

Two future-oriented environmental regulatory programs for petroleum refineries were developed by the ETI project team. These approaches, a risk-based approach (RBB) and a goalbased approach (NPA), strive to meet the potentially conflicting goals of environmental responsibility and economic performance by using pollution prevention and other technologies to do so.

The goal-based approach, because it requires less change to the current system and relies less on the findings of forthcoming scientific and technological research, may be more readily implemented in the near term. The risk-based approach, which requires the development, testing, and acceptance of modeling systems and data on characteristics such as pollutant toxicities, exposure routes, dose-response relationships, and cumulative effects, will likely require more time to implement. However, various recently completed, ongoing, and projected studies on these and related topics will provide much of the information needed to implement the RBB within a 20 -year time frame, which is consistent with the overall project parameters.

In developing the alternative approaches, input was collected from potentially interested parties. Participants in seven workshops - each representing a particular interest group provided constructive criticism, candid observations, and thoughtful suggestions for improving the approaches. Workshop participants generally supported the concept of developing futureoriented alternatives for meeting environmental responsibility and economic performance goals that provide flexibility and accountability. They also said that the ETI refinery project should build on the momentum established to date; the current regulatory system needs to be changed; and the ETI approach, given its integrated format and interaction with stakeholder groups, provides an appropriate format to do so. Workshop participants asked for more details and clarification on many of the elements of the individual approaches and suggested that public participation be integrated into each step. They also suggested that implementing the approaches would require continued input from stakeholders, clear indicators of progress, and the ability to revise elements of the approaches to respond to changing environmental concerns.

Several other government and nongovernment studies support the concepts of regulatory reform reflected in the ETI approaches, with many advocating greater use of risk assessment. In addressing the workshop comments, the project team combined the results of other regulatory reform and related research activities with its own thinking to revise the approaches. Thus, the approaches represent concrete compilations of EPA and other reinvention activities that integrate a variety of reform ideas.

In its draft summary report on the Integrated Risk Project (EPA 1999e), the EPA SAB said that further development of an integrated approach to decision making would help facilitate the transition to a "new phase of environmental protection in the next century." It recommended that the EPA expand the use of tools to reduce risk and to "use them in creative coordinated ways to 


\section{$\Delta$}

reduce multiple risks to multiple receptors in communities ... The best way to test the potential of integrated environmental decision making, identify its weaknesses, improve its methodologies, and gain its benefits is by applying it to real-world problems" (EPA 1999e, p. 2). Further development of one or both of the two approaches identified here could provide a mechanism to conduct such testing.

The revised approaches could now benefit from further comment and eventual pilot testing. Such experimentation, either with a U.S. or foreign refinery that could provide actual data or through a hypothetical case study that could provide modeled data, would yield additional information to further improve and refine the approaches. This testing would also further the iterative and integrated approach of testing ideas, generating feedback, and continually revising the approaches.

Sharing interim thinking with various groups generated the interest of several organizations in tracking or participating in case studies to test the prototypes. For example, through DOE, the project team provided information to the Venezuelan government for a possible test of the prototypes at crude-oil upgrading facilities in that country. Also, some state regulators offered to help launch pilot tests at refineries in their jurisdictions. National environmental groups suggested expanding the prototype approaches to the entire petroleum industry (to reflect a broader, life-cycle approach). The EPA's Regulatory Reinvention Office expressed interest in following the activities as part of its reformed Common Sense Initiative. Perhaps most importantly, several local citizens groups have endorsed the ETI approach, thereby increasing the likelihood of its acceptance and possible implementation.

Finally, developing the future-oriented alternative regulatory programs for petroleum refineries has produced benefits beyond those originally expected. These include exchanging information with public interest groups on scientifically based approaches to environmental regulation and providing lessons learned to the EPA for its broader reinvention efforts. There is also a potential for applying these prototypes to other industrial sectors. 


\section{Section 8 References}

API, 1993, Background Discussion of Total Cost Accounting within Petroleum Operations, American Petroleum Institute, Pollution Prevention Task Force, Washington, D.C., p. 1, July.

ATSDR, 1999, Minimal Risk Levels (MRLs) for Hazardous Substances, Agency for Toxic Substances and Disease Registry, Atlanta, Ga., pp. 1-2, May [http://www.atsdr.cdc.gov/ mrls.htm].

Banks, R.D., and G.R. Heaton, Jr., 1995, “An Innovation-Driven Environmental Policy,” Issues in Science and Technology, pp. 49-50, fall.

BNA, 1999a, "Statement on Peer Review Panel on EPA Mixtures Guidance by Panel Chair Gail Charnley, Released May 21, 1999," Daily Environment Report, No. 101, p. E-2, Bureau of National Affairs, Inc., Washington, D.C., May 26.

BNA, 1999b, "EPA Pursues Performance-Based Measurement for Compliance," The Reinvention Report, p. 10, Bureau of National Affairs, Inc., Washington, D.C., May 19.

BNA, 1999c, "Regulators Approve Eight Projects to be Funded by Environmental Penalties," Daily Environment Report, No. 243, p. A-4, Bureau of National Affairs, Inc., Washington, D.C., Dec. 20.

Browner, C., and F. Hansen, 1997, "Cumulative Risk Assessment Guidance - Phase I Planning and Scoping," interoffice memorandum from Browner (Administrator, U.S. Environmental Protection Agency) and Hansen (Deputy Administrator), to EPA Assistant Administrators, General Counsel, Inspector General, Associate Administrators, Regional Administrators, and Staff Office Directors, July 3.

Butler, J.P., and L.A. Nieves 1997, "Cumulative Risk Assessment Phase of the Chicago Cumulative Risk Initiative," paper prepared by Argonne National Laboratory, Argonne, Ill., for U.S. Environmental Protection Agency, Office of Pollution Prevention and Toxics, Oct.

Butler, J.P., and L.A. Nieves, 1998, Summary of Purpose, Scope, and Technical Approach: Evaluating Cumulative Risks in the Chicago Metropolitan Area, prepared by Argonne National Laboratory, Argonne, Ill., for U.S. Environmental Protection Agency, Office of Pollution Prevention and Toxics, May.

Dosher, J.R., and Carney, J.T., 1994, "Sulfur Increase Seen Mostly in Heavier Fractions of Lower-Quality Crudes,” Oil \& Gas Journal, pp. 43-48, May 23. 
Energy Information Administration, 1984-1998, Petroleum Supply Annual, 1984 through 1998, U.S. Department of Energy, Office of Oil and Gas, Washington, D.C.

Enterprise for the Environment, 1996, Roundtable on Key Elements of the Environmental Management System in Foreign Countries, Washington, D.C., Nov. 6.

EPA: See U.S. Environmental Protection Agency.

GAO: See U.S. Government Accounting Office.

Gow, L.J.A., 1995, “Implementing Sustainability: New Zealand's Experience with Its Resource Management Act," speech by Gow (Acting Secretary for the Environment, Ministry for the Environment, New Zealand), at the New Zealand Embassy, Washington, D.C., June 6 [http://rri.org/gparchive/nz-gows.html].

Hinrichsen, D., 1990, "Integrated Permitting and Inspection in Sweden," pp. 155-160 in Integrated Pollution Control in Europe and North America, N. Haigh and F. Irwin (editors), The Conservation Foundation, Washington, D.C., and the Institute of European Environmental Policy, London, England.

Klee, H., Jr., and Podar, M., 1992, Amoco-U.S. EPA Pollution Prevention Project, Project Summary, Amoco Corporation and U.S. Environmental Protection Agency, Washington, D.C., pp. 1-7, June.

KPMG Milieu, 1993, National Environmental Policy Plan 2, Evaluation of Industry, final report, KPMG Milieu/IVA Tilburg, The Hague, March [http://www.rri.org/gparchive/policyplan.html].

Marcus, A., et al., 1999, Impasse in the Movement toward a New Competence in Environmental Management, prepared by the University of Minnesota under U.S. Environmental Protection Agency Grant R824754, p. 3, Apr. 16.

NESCAUM, 1999, Hazardous Air Pollution Prevention (HAP2) Project Final Report to EPA, Northeast States for Coordinated Air Use Management, Boston, Mass., and Northeast Waste Management Officials' Association, Boston, Mass., p. 1, Feb. 23.

New Jersey Department of Environmental Protection, 1999a, Step 4: Identify Your Processes and Sources, Office of Pollution Prevention and Permit Coordination, Trenton, N.J. [http://www. state.nj.us/dep/opppc/guide/step4.htm].

New Jersey Department of Environmental Protection, 1999b, Air Toxics in New Jersey, toxics release information, Trenton, N.J. [http://www.state.nj.us/dep/airmon/airtoxics].

New Jersey Department of Environmental Protection, 1999c, Step 11: Tracking and Reporting Progress, Office of Pollution Prevention and Permit Coordination, Trenton, N.J. [http://www. state.nj.us/dep/opppc/guide/step11.htm]. 
New Jersey Department of Environmental Protection, 1999d, Pollution Prevention Program, Proposed Readoption with Amendments: N.J.A.C. 7:1K, Office of Pollution Prevention and Permit Coordination, Trenton, N.J., pp. 15 and 17, Nov. 11.

NJDEP: See New Jersey Department of Environmental Protection.

NPC, 1993, U.S. Petroleum Refining - Meeting Requirements for Cleaner Fuels and Refineries, National Petroleum Council, Committee on U.S. Petroleum Refining, Washington, D.C., Aug.

Oil and Gas Journal, 1999, "Fenceline-Monitoring System Solves Big Problem," pp. 61-63, May 24.

Oregon, 1999, Green Permits and the Environmental Management Systems Incentives Project, Oregon Department of Environmental Quality, Portland, Ore., pp. 1 and 4, July update.

PPA, 1996, Alternative Regulatory Pathway: Evaluation Criteria, Pollution Prevention Alliance, Oct. [http://alt-path.com/arp.htm].

Presidential Commission, 1997, Framework for Environmental Health Risk Management, final report, Vol. 1, Presidential/Congressional Commission on Risk Assessment and Risk Management, Washington D.C., Jan. [http://www.riskworld.com/Nreports/1997/risk-rpt/html/ epajana.htm].

Pronk, J., and H. Blankert, 1998, Silent Revolution, Dutch Industry and the Dutch Government Are Working Together for a Better Environment, No. 18204.191, Ministry of Housing, Spatial Planning and the Environment (VROM), The Netherlands, Oct.

Rehbinder, E., 1997, "Environmental Agreements. A New Instrument of Environmental Policy," European University Institute, Robert Schuman Centre for Advanced Studies, San Domenico di Fiesole, Italy, Hean Monnet Chair Paper RSC No. 97/45, Apr. [http://www.iue.it/RSC/WPTexts/rehbinder.html].

RRI, 1999, Case Study: The Netherlands National Environmental Policy Plan, Resource Renewal Institute, San Francisco, Calif. [http://www.rri.org/envatlas/europe/netherlands/nlindex.html].

Seif, J.M., 1995, Secretarial Directive: Regulatory Negotiation, in memo from Seif (Secretary, Commonwealth of Pennsylvania, Department of Environmental Protection, Harrisburg, Penn.) to Deputies and Bureau Directors, Nov. 27 [http:www.dep.state.pa.us/dep/subject/involved/ regneg-guide.html].

Spinello, J.F., 1997, “Case Study: New Jersey — Facility-Wide Permitting Program,” presented at Environmental Regulatory Innovations Symposium, Nov. 5-7 sponsored by Environmental Council of the States and the Minnesota Pollution Control Agency, Minneapolis, Minn. [http://www/pca/state.mn.us/hot/es-nj.html]. 
TNRCC, 1996, Statement on the Regulatory Reform Process, Texas Natural Resource Conservation Commission, Austin, Texas, Apr. [http://www.tnrcc.state.tx.us/comm/ refstate.html].

TNRCC, 1999, Clean Industries 2000, Texas Natural Resource Conservation Commission, Austin, Texas, pp.1-6, July [http://www.tnrcc.state.tx.us/exec/oppr/ci2000/ci2000.html].

U.S. Environmental Protection Agency, 1986, Guidelines for Health Risk Assessment of Chemical Mixtures, Federal Register, Vol. 51, p. 34014, Sept.

U.S. Environmental Protection Agency, 1989, "National Emission Standards for Hazardous Air Pollutants," Federal Register 54:38044, Final Rule, Sept. 14.

U.S. Environmental Protection Agency, 1995a, Profile of the Petroleum Refining Industry, EPA310-R-95-013, Office of Enforcement and Compliance Assurance, p. 52, Sept.

U.S. Environmental Protection Agency, 1995b, An Introduction to Environmental Accounting as a Business Management Tool: Key Concepts and Terms, EPA-742-R-95-001, Office of Prevention, Pesticides, and Toxic Substances, June.

U.S. Environmental Protection Agency, 1995c, The Alternative Dispute Resolution Fact Sheet, Office of Enforcement and Compliance Assurance, May [http://es.epa.gov/oeca/osre/ 950500-2.html].

U.S. Environmental Protection Agency, 1997a, Environmental Cost Accounting for Chemical and Oil Companies: A Benchmarking Study, EPA-742-R-97-004, Office of Prevention, Pesticides, and Toxic Substances, p. 7, June.

U.S. Environmental Protection Agency, 1997b, Applying Environmental Accounting to Electroplating Operations: An In-Depth Analysis, EPA-742-R-97-003, Office of Prevention, Pesticides, and Toxic Substances, May.

U.S. Environmental Protection Agency, 1997c, Merck \& Co.: Final Project Agreement, Merck \& Co., Inc. Stonewall Plant, Jan. 16.

U.S. Environmental Protection Agency, 1997d, Weyerhaeuser Company: Final Project Agreement, Flint River Operations, Jan. 17.

U.S. Environmental Protection Agency, 1998a, minutes of a public meeting of the EPA Science Advisory Board, Environmental Models Subcommittee of the Executive Committee, May 5 and 6 [http://www.epa.gov/science1/ems598ns.htm].

U.S. Environmental Protection Agency, 1998b, Human Health Risk Assessment Protocol for Hazardous Waste Combustion Facilities, EPA-530-D-98-001A, Office of Solid Waste and Emergency Response, pp. 1-14, July. 
U.S. Environmental Protection Agency, 1998c, Sector Facility Indexing Project Progress Report: July 1998, EPA-305-R-98-002, Office of Enforcement and Compliance Assurance, July.

U.S. Environmental Protection Agency, 1998d, Negotiated Rulemaking at the Environmental Protection Agency [http://www.epa.gov/ooaujeag/stakeholders/history.htm].

U.S. Environmental Protection Agency, 1999a, Constructive Resource Engagement Guide: Practical Advice for Dialogue among Facilities, Workers, Communities, and Regulators, EPA745-B-99-008, Office of Prevention, Pesticides, and Toxic Substances, June.

U.S. Environmental Protection Agency, 1999b, Users Manual for OPPT's Risk-Screening Environmental Indicators Model: Version 1.0, Office of Prevention, Pesticides, and Toxic Substances, July 6.

U.S. Environmental Protection Agency, 1999c, Residual Risk Report to Congress, EPA-453/R99-001, Office of Air Quality Planning and Standards, p. 21, March.

U.S. Environmental Protection Agency, 1999d, An SAB Advisory: The National Human Exposure Assessment Survey (NHEXAS) Pilot Studies, EPA-SAB-IHEC-ADV-99-004, Science Advisory Board, Integrated Human Exposure Committee, Feb.

U.S. Environmental Protection Agency, 1999e, Integrated Environmental Decision-Making in the Twenty-First Century: Summary Recommendations - Peer Review Draft, EPA-SAB-EC-99, Science Advisory Board, Integrated Risk Project Steering Committee.

U.S. Environmental Protection Agency, 1999f, “Air Toxics Monitoring Concept Paper,” Office of Air Quality Planning and Standards, p. 1, Apr. 7.

U.S. Environmental Protection Agency, 1999g, Draft Economic Incentive Program Guidance, EPA-452/D-99-001, Office of Air and Radiation, Sept.

U.S. Environmental Protection Agency, 1999h, "Peer Review U.S. Environmental Protection Agency Workshop on Guidance for Conducting Health Risk Assessment of Chemical Mixtures," Federal Register 64(85):23833, May 4.

U.S. Environmental Protection Agency, 1999i, Project XL 1999 Comprehensive Report, EPA100-R-99-008, Office of the Administrator, p. iii, Oct.

U.S. Environmental Protection Agency, 1999j, Project XL Stakeholder Involvement: A Guide for Project Sponsors and Stakeholders, EPA-100-F-99-001, Office of Reinvention, Feb.

U.S. Environmental Protection Agency, 1999k, Index of Supplemental Environmental Projects, Region III, Dec. 20 [http://www.epa.gov/reg3ecej/enforcement/sepindex.htm, updated quarterly].

U.S. Environmental Protection Agency, 19991, Aiming for Excellence, EPA-100-R-99-006, Office of the Administrator, July. 
U.S. Environmental Protection Agency, 1999m, Guidance for Conducting Health Risk Assessment of Chemical Mixtures, NCEA-C-0148, Office of Research and Development, National Center for Environmental Assessment, Risk Assessment Forum Technical Panel, p. 2, Apr.

U.S. Government Accounting Office, 1997a, Air Pollution: Overview and Issues on Emissions Allowance Trading Programs, GAO/T-RCED-97-183, statement of P.F. Guerrero (Director, Environmental Protection Issues, Resources, Community, and Economic Development Division, GAO), p. 12, July.

U.S. Government Accounting Office, 1997b, EPA's and States' Efforts to "Reinvent" Environmental Regulation, GAO/T-RCED-98-33, statement of P.F. Guerrero (Director, Environmental Protection Issues, Resources, Community, and Economic Development Division, GAO), p. 2, Nov. 4.

U.S. Government Accounting Office, 1997c, Challenges Facing EPA's Efforts to Reinvent Environmental Regulation, GAO/RCED-97-155, report to Congressional requesters, p. 50, July.

Wood, D.B., 1997, "Blueprint for Cleaner Skies under Fire," The Christian Science Monitor, July 28.

Woods, R., 1995, "Regulatory Negotiation: Early Involvement Ensures Rules People Can Live with," BBB Solution 5(3):1, Council of Better Business Bureaus, Dispute Resolution Division [http://www.bbb.org/complaints/regneg.html]. 


\section{Appendix A Recent Regulatory Reform Efforts}

Table A.1 Recent Regulatory Reform Efforts

\begin{tabular}{|c|c|c|c|}
\hline Reform Effort & Goal & Features & $\begin{array}{l}\text { Relationship to } \\
\text { ETI Program }\end{array}$ \\
\hline $\begin{array}{l}\text { Common Sense } \\
\text { Initiative (CSI) }\end{array}$ & $\begin{array}{l}\text { To bring government officials, } \\
\text { environmental groups, and } \\
\text { industry together to create } \\
\text { industry-specific strategies to } \\
\text { work toward "cleaner, cheaper, } \\
\text { and smarter" ways to achieve } \\
\text { environmental protection } \\
\text { through consensus-based } \\
\text { decision making. }\end{array}$ & $\begin{array}{l}\text { Focuses on six industrial sectors, } \\
\text { one of which is petroleum } \\
\text { refining. } \\
\text { Incorporates formal stakeholder } \\
\text { process. }\end{array}$ & $\begin{array}{l}\text { Initiative helps focus } \\
\text { efforts on issues to be } \\
\text { addressed by the } \\
\text { regulatory reform effort. }\end{array}$ \\
\hline $\begin{array}{l}\text { Effluent Trading } \\
\text { in Watersheds }\end{array}$ & $\begin{array}{l}\text { To implement effluent trading } \\
\text { on a national scale as a cost- } \\
\text { effective approach for reducing } \\
\text { water pollution. }\end{array}$ & $\begin{array}{l}\text { Dischargers that have reduced } \\
\text { pollution below the minimum level } \\
\text { required to meet water quality } \\
\text { standards can sell their excess } \\
\text { pollution reductions to other } \\
\text { dischargers within the same } \\
\text { watershed. }\end{array}$ & $\begin{array}{l}\text { Program provides basis } \\
\text { for some of the trading } \\
\text { options envisioned in one } \\
\text { of the approaches. }\end{array}$ \\
\hline $\begin{array}{l}\text { Environmental } \\
\text { Leadership } \\
\text { Program (ELP) }\end{array}$ & $\begin{array}{l}\text { To recognize and provide } \\
\text { incentives to facilities } \\
\text { developing and demonstrating } \\
\text { innovative approaches to } \\
\text { establishing and ensuring } \\
\text { compliance with environmental } \\
\text { requirements. }\end{array}$ & $\begin{array}{l}\text { Innovative approaches can include } \\
\text { implementation of an environmental } \\
\text { management system, routine audits } \\
\text { through third-party verification or } \\
\text { self-certification, sharing } \\
\text { environmental performance } \\
\text { information with the public, and } \\
\text { pollution prevention practices. }\end{array}$ & $\begin{array}{l}\text { Concepts apply to } \\
\text { program, and specific } \\
\text { approaches may be } \\
\text { incorporated into the } \\
\text { multimedia regulatory } \\
\text { program. }\end{array}$ \\
\hline $\begin{array}{l}\text { Open Market } \\
\text { Air Emission } \\
\text { Trading }\end{array}$ & $\begin{array}{l}\text { To expand current air pollution } \\
\text { trading program to include a } \\
\text { more flexible approach for } \\
\text { complying with ozone air quality } \\
\text { standards. }\end{array}$ & $\begin{array}{l}\text { Encourages use of technologies } \\
\text { prior to required phase-in schedules. } \\
\text { Provides incentives for reducing } \\
\text { emissions to levels below current } \\
\text { requirements. Allows emissions } \\
\text { trading to occur without prior } \\
\text { U.S. Environmental Protection } \\
\text { Agency (EPA) approval as long as } \\
\text { reporting and public health } \\
\text { standards are met. }\end{array}$ & $\begin{array}{l}\text { Program provides basis } \\
\text { for some of the trading } \\
\text { options envisioned in one } \\
\text { of the approaches. }\end{array}$ \\
\hline $\begin{array}{l}\text { Performance- } \\
\text { Based } \\
\text { Reduction of } \\
\text { National } \\
\text { Pollutant } \\
\text { Discharge } \\
\text { Elimination } \\
\text { System } \\
\text { (NPDES) Permit } \\
\text { Monitoring } \\
\text { Frequencies }\end{array}$ & $\begin{array}{l}\text { To reduce monitoring } \\
\text { requirements for facilities with } \\
\text { long records of excellent } \\
\text { performance. }\end{array}$ & $\begin{array}{l}\text { Allows reductions in monitoring } \\
\text { frequency as required by the } \\
\text { existing permit. Provides for } \\
\text { additional reductions in monitoring } \\
\text { frequency if permittees agree to } \\
\text { collect and provide additional } \\
\text { ambient monitoring information. }\end{array}$ & $\begin{array}{l}\text { Concepts may be } \\
\text { incorporated into the } \\
\text { compliance assurance } \\
\text { provisions. }\end{array}$ \\
\hline
\end{tabular}


Table A.1 Recent Regulatory Reform Efforts (Cont.)

\begin{tabular}{|c|c|c|c|}
\hline Reform Effort & Goal & Features & $\begin{array}{l}\text { Relationship to } \\
\text { ETI Program }\end{array}$ \\
\hline $\begin{array}{l}\text { Permit } \\
\text { Improvement } \\
\text { Team (PIT) }\end{array}$ & $\begin{array}{l}\text { To identify improvements in air, } \\
\text { waste, and water permitting } \\
\text { programs to provide more } \\
\text { environmental protection at less } \\
\text { cost. }\end{array}$ & $\begin{array}{l}\text { Relies on public, performance- } \\
\text { based permitting to establish a } \\
\text { defined level of performance to be } \\
\text { achieved by permittee. Provides the } \\
\text { public with adequate information to } \\
\text { monitor the permitting process and } \\
\text { compliance of permitted facility. }\end{array}$ & $\begin{array}{l}\text { Findings of PIT may be } \\
\text { incorporated with respect } \\
\text { to permits. }\end{array}$ \\
\hline Project XL & $\begin{array}{l}\text { To test innovative ways to } \\
\text { achieve better and more cost- } \\
\text { effective public health and } \\
\text { environmental protection } \\
\text { through site-specific pilot } \\
\text { projects. }\end{array}$ & $\begin{array}{l}\text { The EPA evaluation criteria include, } \\
\text { among others, environmental } \\
\text { performance that is superior to what } \\
\text { would be achieved through } \\
\text { compliance with current and } \\
\text { reasonably anticipated future } \\
\text { regulation, cost savings, paperwork } \\
\text { reduction, and stakeholder support. }\end{array}$ & $\begin{array}{l}\text { Experience with this } \\
\text { program provides lessons } \\
\text { learned for developing the } \\
\text { alternative ETI } \\
\text { approaches. }\end{array}$ \\
\hline $\begin{array}{l}\text { Regulatory } \\
\text { Negotiation }\end{array}$ & $\begin{array}{l}\text { To apply consensus-based } \\
\text { decision-making techniques in } \\
\text { rule making. }\end{array}$ & $\begin{array}{l}\text { The EPA and representatives of all } \\
\text { major groups affected by a rule } \\
\text { attempt to reach agreement on } \\
\text { regulatory requirements in order to } \\
\text { increase public acceptability and } \\
\text { decrease litigation. }\end{array}$ & $\begin{array}{l}\text { This was used as a basis } \\
\text { for one of the approaches. }\end{array}$ \\
\hline $\begin{array}{l}\text { Enterprise for } \\
\text { the Environment }\end{array}$ & $\begin{array}{l}\text { To engage environmentalists, } \\
\text { business, labor, government } \\
\text { officials, and others in a policy } \\
\text { dialogue aimed at rebuilding a } \\
\text { consensus on the strategic } \\
\text { direction of environmental } \\
\text { policy. }\end{array}$ & $\begin{array}{l}\text { Studies whether policy reforms are } \\
\text { needed to shift the current } \\
\text { regulatory system toward one that is } \\
\text { more flexible and performance- } \\
\text { based and uses market } \\
\text { mechanisms and alternative } \\
\text { enforcement and compliance } \\
\text { approaches to achieve } \\
\text { environmental protection. }\end{array}$ & $\begin{array}{l}\text { Program bolsters support } \\
\text { for ETI by contributing to } \\
\text { the debate on how best to } \\
\text { implement more cost- } \\
\text { effective and efficient } \\
\text { approaches to } \\
\text { environmental protection. }\end{array}$ \\
\hline $\begin{array}{l}\text { Reinventing } \\
\text { Environmental } \\
\text { Regulations }\end{array}$ & $\begin{array}{l}\text { To be one of the earliest (March } \\
\text { 1995) formal recognitions of the } \\
\text { need for regulatory reform in } \\
\text { the environmental arena. }\end{array}$ & $\begin{array}{l}\text { Lists } 10 \text { principles for reinventing } \\
\text { environmental regulations (e.g., } \\
\text { regulations must be performance } \\
\text { based, provide maximum flexibility } \\
\text { in means for achieving } \\
\text { environmental goals, and require } \\
\text { accountability for results) and } 35 \\
\text { high-priority actions. }\end{array}$ & $\begin{array}{l}\text { Many of the principles } \\
\text { (e.g., flexibility, pollution } \\
\text { prevention, collaborative } \\
\text { decision making, market } \\
\text { incentives) are reflected in } \\
\text { the ETI approaches. }\end{array}$ \\
\hline $\begin{array}{l}\text { Executive Order } \\
12866\end{array}$ & $\begin{array}{l}\text { To contain the administration's } \\
\text { principles for regulatory } \\
\text { planning and review. }\end{array}$ & $\begin{array}{l}\text { Regulatory goals should be } \\
\text { expressed in terms of outcomes } \\
\text { rather than design standards or } \\
\text { methods of compliance. }\end{array}$ & $\begin{array}{l}\text { The ETI approaches } \\
\text { embody the concept of } \\
\text { performance-based goals } \\
\text { and flexibility to promote } \\
\text { technology innovation and } \\
\text { use. }\end{array}$ \\
\hline
\end{tabular}


Table A.1 Recent Regulatory Reform Efforts (Cont.)

\begin{tabular}{|c|c|c|c|}
\hline Reform Effort & Goal & Features & $\begin{array}{l}\text { Relationship to } \\
\text { ETI Program }^{\mathrm{a}}\end{array}$ \\
\hline $\begin{array}{l}\text { National } \\
\text { Academy of } \\
\text { Public } \\
\text { Administration } \\
\text { (NAPA) }\end{array}$ & $\begin{array}{l}\text { To review the EPA's role in } \\
\text { setting the nation's } \\
\text { environmental priorities. } \\
\text { (Congress asked NAPA [a } \\
\text { nonprofit, nonpartisan } \\
\text { organization] to do this.) }\end{array}$ & $\begin{array}{l}\text { NAPA found that more rational, less } \\
\text { costly strategies for protecting the } \\
\text { environment are needed if } \\
\text { environmental progress is to } \\
\text { continue. It also identified the need } \\
\text { for profound changes in the legal } \\
\text { foundation, to support flexibility and } \\
\text { accountability, and in EPA's } \\
\text { management structure, to remove } \\
\text { the barriers from its various media- } \\
\text { specific programs. }\end{array}$ & $\begin{array}{l}\text { The ETI alternatives } \\
\text { embrace multimedia } \\
\text { approaches. NAPA's } \\
\text { recommendations for } \\
\text { statutory change are } \\
\text { consistent with ETI's } \\
\text { future-oriented } \\
\text { approaches, elements of } \\
\text { which can conflict with } \\
\text { current statutes. }\end{array}$ \\
\hline $\begin{array}{l}\text { EPA Office of } \\
\text { Reinvention }\end{array}$ & $\begin{array}{l}\text { To develop initiatives to } \\
\text { address environmental } \\
\text { problems that have yet to be } \\
\text { solved through the current } \\
\text { system. }\end{array}$ & $\begin{array}{l}\text { Recent report, Aiming for } \\
\text { Excellence (EPA 1999I), commits } \\
\text { agency to pursue more reinvention } \\
\text { activities with regard to incentives } \\
\text { and to promote environmental } \\
\text { management systems. }\end{array}$ & $\begin{array}{l}\text { Ideas are consistent with } \\
\text { ETI approaches, except } \\
\text { that EPA reinvention time } \\
\text { frame is } 12-18 \text { months } \\
\text { versus } 20 \text {-plus years for } \\
\text { ETI approaches }\end{array}$ \\
\hline
\end{tabular}

a ETI is the U.S. Environmental Technology Initiative.

b EPA, 1999l, Aiming for Excellence, EPA-100-R-99-006, U.S. Environmental Protection Agency, Office of the Administrators, July 11. 
A 


\section{Appendix B \\ Guidelines and Principles for Developing Alternative Regulatory Approaches}

Table B.1 Guidelines for Developing Alternative Regulatory Approaches

\begin{tabular}{|ll|}
\hline \multicolumn{1}{|c|}{ Guideline } & \multicolumn{1}{c|}{ Explanation } \\
\hline $\begin{array}{l}\text { 1. Petroleum products will continue to play an } \\
\text { important role in U.S. society. }\end{array}$ & $\begin{array}{l}\text { For the foreseeable future, petroleum products are certain to } \\
\text { play an important role in the U.S. economy and society. }\end{array}$ \\
$\begin{array}{l}\text { 2. Regulatory approaches will be limited to } \\
\text { refinery activities. }\end{array}$ & $\begin{array}{l}\text { Approaches developed through this project will focus on } \\
\text { petroleum refineries and closely associated operations. The } \\
\text { extraction of crudes and use of finished products will not be } \\
\text { addressed. }\end{array}$ \\
$\begin{array}{l}\text { 3. Regulatory approaches shall ensure protection } \\
\text { of human health and the environment. }\end{array}$ & $\begin{array}{l}\text { Alternative regulatory approaches will be more efficient in } \\
\text { protecting human health and the environment by doing things } \\
\text { faster, smarter, and cheaper. }\end{array}$ \\
\hline
\end{tabular}


Table B.2 Principles for Developing Alternative Regulatory Approaches

\author{
Principle \\ Explanation
}

1. Regulatory approaches shall be rooted in the ethic of pollution prevention.

2. Regulatory approaches shall encourage, stimulate, and reward innovation.

3. Regulatory approaches shall focus on reducing the net environmental effect associated with refinery operations.

4. Administrative and information requirements under alternative regulatory approaches shall not inhibit innovation or performance.

5. Regulatory approaches shall involve stakeholders in the regulatory process.

6. Regulatory approaches should reflect site-specific conditions and environmental issues.

7. Regulatory approaches should not adversely impact the economic performance of petroleum refineries.
Regulations governing tomorrow's refineries shall be rooted in the ethic of pollution prevention, which strives to reduce, up front, the potential risks to human health and the environment. A fundamental goal of pollution prevention is to continually strive to reduce overall emissions and waste generation, with a focus on reducing emissions and wastes known to present risks to human health and the environment.

Alternative regulatory approaches shall encourage, stimulate, and reward the application and use of administrative and technological innovations for improving process design, operations and maintenance, and pollution control technology performance. Prescriptive requirements shall be minimized. Alternative regulatory approaches shall leave maximum flexibility for innovation. Prescriptive criteria or implicit preference for an approach or technology should be avoided because of the possibility of restricting or discouraging innovation and improvement.

Alternative regulatory approaches shall focus on the notion that refinery operations should pursue activities that have a lesser or reduced effect on human health and the environment. This lesser or reduced effect is a function of multiple attributes. A specific pollution prevention option, for example, may result in the generation of another waste; the issue is whether this secondary waste is more or less harmful to human health and the environment than the original waste. The scale (local versus global) and temporal reversibility aspects of impacts are other examples of attributes that have an impact on net environmental effects. Although analyses shall consider all benefits and shortcomings of alternate approaches, the focus shall be on reducing the net environmental effect associated with a refinery. (Occupational health and safety are not within the scope of the project.)

Wherever possible, these requirements shall be consistent and streamlined. These requirements should leave maximum flexibility for technological or other innovations.

The development of a new regulatory program should be an open process. The participation of stakeholders in actions such as regulatory permitting and reporting and other key approach components will be encouraged.

Site-specific environmental conditions (such as climate, geology, hydrology, and potentially exposed populations) vary significantly across the United States. These conditions can affect the performance of technology and operations. Alternative regulatory approaches will need to incorporate this variability.

Recognizing the social and economic importance of the petroleum refining industry to the United States, alternative regulatory approaches should not result in deteriorating economic performance by refineries. Regulatory approaches should not place undue burden on refineries, which might result in de facto subsidies for alternative fuels or technologies. 


\section{Appendix C Environmental Laws and Regulations Affecting Petroleum Refineries}

\section{Table C.1 Environmental Laws and Regulations Affecting Petroleum Refineries ${ }^{a}$}

\begin{tabular}{|c|c|c|}
\hline Statute & Current Regulatory Programs & $\begin{array}{c}\text { Recent and Impending } \\
\text { Regulations }\end{array}$ \\
\hline Clean Air Act (CAA) & $\begin{array}{l}\text { Stationary Source Regulations } \\
\text { - New Source Review and New Source } \\
\text { Performance Standards } \\
\text { - National Emissions Standards for } \\
\text { Hazardous Air Pollutants (NESHAP) }\end{array}$ & $\begin{array}{l}\text { - } \text { Title V operating permit program } \\
\text { - } \quad \text { NESHAP } \\
\text { Chemical accident prevention and } \\
\text { risk management } \\
\text { - Plantwide applicability limits under } \\
\text { new source review }\end{array}$ \\
\hline Clean Water Act (CWA) & $\begin{array}{l}\text { - National Pollutant Discharge Elimination } \\
\text { System (NPDES) Program } \\
\text { - Storm water discharges }\end{array}$ & $\begin{array}{l}\text { Revision of effluent guidelines for } \\
\text { petroleum refining sector }\end{array}$ \\
\hline $\begin{array}{l}\text { Resource Conservation } \\
\text { and Recovery Act (RCRA) }\end{array}$ & $\begin{array}{l}\text { - Standards for accumulation, manifesting, } \\
\text { and record keeping } \\
\text { - Identification of hazardous waste } \\
\text { - Treatment, storage, and disposal facility } \\
\text { permits for hazardous waste } \\
\text { - Restrictions on land disposal } \\
\text { - Standards for managing used oil } \\
\text { - Emission standards for tanks and containers } \\
\text { - Underground storage tanks } \\
\text { - Corrective action program }\end{array}$ & $\begin{array}{l}\text { - Recovered oil exemption } \\
\text { - Rotential listing of waste streams } \\
\text { Rule for hazardous waste } \\
\text { identification }\end{array}$ \\
\hline $\begin{array}{l}\text { Safe Drinking Water Act } \\
\text { (SDWA) }\end{array}$ & $\begin{array}{l}\text { - Underground Injection Control program } \\
\text { - Sole source aquifer program }\end{array}$ & \\
\hline $\begin{array}{l}\text { Comprehensive } \\
\text { Environmental Response, } \\
\text { Compensation, and } \\
\text { Liability Act (CERCLA) }\end{array}$ & $\begin{array}{l}\text { - Regulations on reporting releases of } \\
\text { hazardous substances } \\
\text { - Hazardous substance response } \\
\text { (e.g., removal actions) } \\
\text { - Emergency Planning and Community Right- } \\
\text { To-Know Act notification requirements and } \\
\text { toxic release inventory (TRI) reporting } \\
\text { requirements }\end{array}$ & $\begin{array}{l}\text { Expansion of chemicals reported } \\
\text { under toxic release inventory } \\
\text { Expansion of requirements under } \\
\text { TRI for chemical use data }\end{array}$ \\
\hline Oil Pollution Act of 1990 & $\begin{array}{l}\text { Spill prevention control and countermeasures } \\
\text { plan }\end{array}$ & \\
\hline
\end{tabular}

a Regulations are those that affect refinery operations only. Regulations that pertain to mobile sources and fuel specifications, although they are significant for refineries, are not the topic of this effort and are excluded from this table. 
A 


\section{Appendix D Goals and Indicators for Regulatory Options}

\section{GOALS}

1. Economic performance. Regulatory approaches should not weaken the economic performance of U.S. refineries.

2. Environmental responsibility. Regulatory approaches should provide for the protection of public health and the environment.

3. Pollution prevention technology innovation and use. Pollution prevention technology should be developed and used to help meet the goals of economic performance and environmental responsibility.

\section{INDICATORS}

\section{Economic Performance}

1. Reduce environmental capital costs. Environmental capital costs for refinery regulations and for product quality (cleaner fuels) represent more than $40 \%$ of the total capital expenditures that the refining industry expects to have made between 1991 and 2000 (NPC 1993). If all other factors remain equal, a regulatory program that reduces capital costs for environmental requirements will improve economic performance. Operating and maintenance costs include both fixed costs (for personpower, maintenance, taxes, and insurance) and variable costs (for fuel, power, catalysts, and chemicals). The regulatory approaches identified might require less in capital costs but more in operating costs. For example, a performance-based approach might result in a higher rate of increase for monitoring costs than for capital costs. Similarly, approaches that encourage recycling rather than disposal might result in higher operating costs.

2. Reduce environmental administrative costs. The following activities could lead to reduced environmental administrative costs:

- Streamline environmental administrative processes. An example is a multimedia program that helps to relax or eliminate requirements or prohibitions associated with one environmental medium in return for additional control in another medium.

- Decrease record keeping and reporting. Regulatory approaches that consolidate record keeping and reporting and provide for synergistic interactions with relevant agencies can streamline administrative processes. 
- Reduce permitting requirements and costs. Current regulatory programs require detailed permitting procedures for new plants, modifications to existing plants, and new technology use. Reductions in these requirements can streamline administrative processes.

- Make program introduction and implementation easier. Given that new program start-up and learning costs may be significant, an alternative regulatory program, once in place, should be clearly understandable and require minimal interpretation by the legal profession.

3. Reduce environmental regulatory cost uncertainties. Uncertainties in projecting environmental regulatory costs can be significant and can weaken overall economic performance. Implementation of unanticipated regulations can cause costs to increase more significantly than they would if implementation had been anticipated.

\section{Environmental Responsibility}

4. Reduce quantity of residuals produced. Residuals that are not generated in the first place cannot be incorporated into other products or residuals, will not require treatment or disposal (which could result in cross-media or long-term environmental or health impacts), and will not be released into the environment, where they could cause further harm.

5. Reduce quantity of residuals released to the environment. This indicator allows for recycling and other controls that limit the amount of residuals entering the environment.

6. Reduce toxicity of residuals produced. Tracking reductions in the production of toxic residuals, as a subset of all residuals, provides data necessary for assessing risks to public health and the environment. When all other factors remain equal, a reduction in the production of toxic residuals will reduce risks more than an equal reduction in the production of nontoxic residuals will.

7. Reduce toxicity of residuals released. Assessing the reduction in toxicity of residuals released to the environment provides an indication of risk. It also allows for considering the reduction of residuals, which, while still produced, are not released to the environment because of recycling or end-of-pipe treatments.

8. Use fewer nonfeedstock toxics. If fewer nonfeedstock toxics are used, the potential for toxics to be released to the environment will be reduced. (Use of feedstocks, which always have toxic components, cannot be reduced. Therefore, reduced use of toxics depends on reduced use of nonfeedstock toxics.)

9. Incorporate public participation. An important aspect of environmental responsibility is to integrate community concerns in environmental compliance programs while the programs are being developed. This indicator addresses stakeholder involvement and hazard communication. By providing the public with accurate, complete, and understandable 
information, the programs allow people to make informed judgments on the degree of health risk and to compare those risks with other risks. An approach that incorporates the community into the development process is assumed to meet this indicator of environmental responsibility.

\section{Pollution Prevention Technology Innovation and Use}

10. Promote penetration of new technologies into the refinery industry. This indicator incorporates several elements:

- Allow sufficient time for implementation. End-of-pipe controls often do not require detailed reengineering procedures. In contrast, pollution prevention technologies can require rethinking of entire production processes, requiring greater investments of time and money.

- Allow for failure of new technology used in good faith. This element addresses the issue of how to treat new environmental technologies that were designed and developed in good faith but failed to accomplish their goals. This element can be demonstrated if the program is structured to provide flexibility in the event of such failures.

- Provide incentives for new technology development and use. The regulatory program should include identifiable components that demonstrate the flexibility needed to give companies the incentive to develop and use new pollution prevention technology.

- Encourage use of new technology. Approaches that require or imply the use of new technologies as part of the regulatory program would meet this indicator.

11. Reduce reliance on end-of-pipe technologies. The command-and-control approach to environmental regulatory programs has resulted in technology lock-in. Technology lock-in is the low-risk (from an economic perspective) reliance on previously approved approaches and equipment for meeting end-of-pipe discharge requirements.

\section{REFERENCE}

NPC, 1993, U.S. Petroleum Refining - Meeting Requirements for Cleaner Fuels and Refineries, National Petroleum Council, Committee on U.S. Petroleum Refining, Washington, D.C., Aug. 
A 


\section{Appendix E Summary of Workshop Comments}

\section{GUIDELINES AND PRINCIPLES}

- Do not imply that economics is more important than the environment

- Guidelines and principles may be too limiting; include product use as well as refinery operations

- Increased flexibility implies increased accountability; address accountability

- Rephrase or eliminate words that appear vague or could be considered as providing potential loopholes (e.g., protect, issues)

- Include worker risk

- Include accidental risks

- Address impacts of noise, flares, and odors

\section{CLARIFICATIONS AND BETTER DEFINITIONS OF TERMS}

- Clarify the term "flexibility" from a compliance perspective

- Define "streamlined reporting" (should not mean less reporting but rather that reliable, meaningful data must be available to stakeholders on a periodic basis)

- Define "better environmental performance"

- Explain how the transition from today's regulatory structure will be achieved

\section{COMPONENTS}

- Add component at beginning to establish need

- Include setting goals while setting release limits; goals should include credibility and public disclosure

- Add component at end for performance evaluation

- Include accountability when assuring compliance

\section{PROTOTYPE DESIGN}

- Address perception that approaches may weaken or appear to weaken existing regulations; help stakeholders understand tradeoffs from the existing system

- Ensure that approaches provide both environmental and economic benefits, clarify economic benefits to refiners, show how overall costs will be reduced, and provide for measurement of overall costs (a reason is because it is unlikely that a refiner would adopt any approach unless it paid back the capital investment or significantly reduced compliance costs)

- Explain how performance will be evaluated; develop environmental indicators 
- Expand and develop public participation:

- Incorporate public participation into all components (baseline, setting limits, compliance assurance)

- Define roles, rules, responsibilities, selection process, and types of forums; define who "sits at the table" and who makes the decisions

- Define what "stakeholder input" means (e.g., do stakeholders have sign-off approval; do they have an opportunity to "have their say")

- Explain how public participation is ensured when resources are limited

- Educate stakeholder groups (some may have a limited knowledge of risk or misunderstand risk and how it can be considered when setting standards)

- Identify who selects the participants

- Include providing public access to information as part of involving stakeholders

- Specify how participants will know when agreement has been reached

- Identify who (e.g., everyone at the table) will involved in negotiations

- Identify the roles of elected officials

\section{COMMENTS COMMON TO BOTH APPROACHES}

\section{Implementation}

- Provide concrete examples to increase the probability that refineries will "buy in"

- Identify the "hows" (failure to do so is the reason that so many new approaches have not been adopted)

- Identify resource requirements (state regulators need resources and training; the development of baseline information will be expensive; obtaining regulator/citizen/refinery agreement will be difficult; refineries do not have the data necessary to implement the new approaches)

- Provide clear statutory authority to help the U.S. Environmental Protection Agency foster these approaches

- Identify how to achieve protection from suits filed by citizens.

\section{Establishing Baseline}

- Identify not only residuals but also sources (to help identify pollution prevention opportunities)

- Define a point in time at which the baseline would be established

- Explain if approaches are designed to control all facility-specific residuals or to focus on selected residuals (if they are to focus, explain screening methodology) 


\section{Setting Limits}

- Identify what process will be used for contesting limits

- Identify who will receive information developed as part of the process of setting limits

\section{Assuring Compliance}

- Define "assessing penalties" (are they more than a financial penalties and, if so, what are they)

- Identify how the results for two refineries with two different sets of goals can be compared (need for performance indicators), because even though programs are site-specific, people will want to compare results

- Identify how results will be measured and characterized

- Identify what incentives and disincentives will be used to assure compliance

- Provide details on trading, particularly with respect to toxics

- Clearly define how enforcement will occur, what will be enforceable, and how regulators can be assured that a refinery is compliant (regulators do not want to rely solely on information provided to them by refineries)

- Identify what will happen if, despite compliance assurance methods, refineries fail to meet the release limits

\section{COMMENTS SPECIFIC TO RISK-BASED BUBBLE (RBB)}

\section{Establishing Baseline}

- Explain how (if) information on contextual meaning of residuals will be made publicly available (such information would help in establishing the baseline and appropriate release limits)

- Explain how residuals and other environmental information from nearby sources will be obtained and used in characterizing the environment surrounding the refinery

- Explain quality assurance/quality control procedures for independently verifying information solicited from stakeholder groups in characterizing the environment

- Define the process for determining residuals of concern, including the establishment of criteria for determining residuals of concern

- Explain design protocols for establishing the baseline

\section{Setting Limits}

- Explain who sets the goals

- Explain how acceptable risk levels are determined, identify who determines acceptable risk levels, address whether all communities should use the same risk goal (e.g., 1 in 1,000,000) or whether they should establish different risk goals 
- Explain how protocols for setting limits are designed

- Explain the process for considering or planning with other industries to establish risk-based limits

- Define "cumulative" (consider cumulative effects in setting limits today even if an effect may not be experienced for 20 years; while the program assumes that the baseline characterization would incorporate releases and effects of other sources, disagreements regarding the actual sources of releases may affect actual implementation)

- Define conditions for reopeners (e.g., is consensus among all parties required)

- Explain how distinctions between acute risk and chronic risk would be addressed

- Define how quality is ensured for modeling

- Identify sources of data for model input and how such data would be obtained

\section{Assuring Compliance}

- Explain how the initial allocations for trading would be made for each pollutant for each facility, particularly when other sources are nearby (trading within a facility is viewed as acceptable; trading across facilities raises concerns)

- Explain how pollutants subject to trading will be identified, how trading will occur, and what tools will be developed and used to implement cross-pollutant and cross-media trading

- Explain who is responsible for deciding how and what chemicals are traded and how they are traded (how chemicals are traded could lead to disparate impacts on different populations)

- Address the temporal aspects of trading (i.e., flexibility is perceived to come first, with environmental benefits secondary)

- Explain whether caps would be set for pollutants traded across media, and if they are, how they would be set

- Specify the type(s) of monitoring (communities generally prefer fence-line monitoring and refineries generally oppose it; citizens stress the need for increased monitoring in strategic locations, and citizens can be trained to operate the equipment)

- Explain how monitoring results would be made publicly available (doing so will help assure compliance)

\section{COMMENTS SPECIFIC TO NEGOTIATED PERFORMANCE AGREEMENT (NPA)}

\section{Establishing Baseline}

- Explain what is meant by costs and how the identification of costs pertains to the negotiated agreement (include economic externalities and environmental impact costs as well as environmental management costs)

- Use total cost accounting; examine life-cycle considerations

- Explain how confidentiality of cost information will be protected

- Place releases in context (e.g., show how a release affects health and the environment; show how it compares with a previous release) 
- Clarify whether the baseline is current operating experience or current regulations (using current regulations as a starting point may be unsatisfactory because current regulations are not sufficiently comprehensive)

- Explain whether all reductions from the baseline are treated equally or if residuals are ranked in some way

- Explain how refiners will be prevented from "adjusting" baseline numbers to show progress relative to that baseline

- Provide for continuous improvement (i.e., ensure that emissions can be reduced to below the baseline; address concern that baseline is static and is used as a target)

\section{Setting Limits}

- Explain how reductions made before the date when the baseline was established are considered when setting limits

- Ensure that measurement units reflect the impacts they are intended to address (tons per year might allow significant short-term impacts)

- Define the lifespan of the NPA; explain how the NPA time period relates to existing terms for permits under which the refinery is currently operating

- Identify specifically who is involved in the negotiating

- When anticipated regulations are used to set limits, explain what happens if the regulations, when promulgated, are more stringent than the ones on which the limits were based

- Explain how early reductions are considered in setting limits

\section{Assuring Compliance}

- Define what (if any) milestones will need to be met before the end of the negotiated performance period

- Address the timing of penalties (e.g., determine if penalties can be assessed at any point during the NPA or only at the end of the period, and, if they can be assessed at any point, explain the mechanism for such assessments)

- Explain what is meant by "compensating affected interests" and provide specific examples

- Reconsider whether disputes should be resolved by using an arbitration panel (while common ground may be reached, that common ground may not be protective of human health)

- Explain how the NPA controls for the possibility that goals will be set too high and therefore not met and also how refiners would be penalized in such cases 
A 


\title{
Appendix F Studies and Efforts That Support the Concepts and Provisions of the Risk-Based Bubble
}

\author{
Table F.1 Studies and Efforts That Support the Concepts and Provisions of the \\ Risk-Based Bubble (RBB)
}

\begin{tabular}{|c|c|c|}
\hline Effort/Organization & Goal/Objective & Congruence with/Support for RBB \\
\hline $\begin{array}{l}\text { California Air Toxics } \\
\text { Program }\end{array}$ & $\begin{array}{l}\text { To examine risks attributable to specific } \\
\text { chemicals, and for those risks that warrant } \\
\text { action, to examine all sources to determine a } \\
\text { cost-effective strategy for reducing emissions } \\
\text { and exposures. }\end{array}$ & $\begin{array}{l}\text { Embodies RBB concepts of cost-effective } \\
\text { means for reducing emissions and } \\
\text { exposures to pollutants identified as } \\
\text { contributing to total cumulative risk. }\end{array}$ \\
\hline $\begin{array}{l}\text { Chicago Cumulative } \\
\text { Risk Initiative }\end{array}$ & $\begin{array}{l}\text { To measure cumulative risks to metropolitan } \\
\text { area residents. }\end{array}$ & $\begin{array}{l}\text { Uses environmental loading profile (similar } \\
\text { to RBB baseline inventory) and } \\
\text { workshops, develops a methodology to } \\
\text { approximate cumulative risks for local } \\
\text { residents, and implements pollution } \\
\text { prevention activities, all of which are } \\
\text { reflected in RBB. }\end{array}$ \\
\hline $\begin{array}{l}\text { Contaminated Surface } \\
\text { Waterway Cleanup } \\
\text { Initiative (proposed } \\
\text { August 1999) }\end{array}$ & $\begin{array}{l}\text { To make all U.S. waters safe for swimming and } \\
\text { fishing by using total maximum daily load } \\
\text { requirements and site-specific approaches. } \\
\text { Would establish caps for pollution entering a } \\
\text { given waterway, require timetables for } \\
\text { implementing emission reduction plans, and } \\
\text { allow for trading. }\end{array}$ & $\begin{array}{l}\text { Consistent with the RBB approach of } \\
\text { examining total cumulative impacts in } \\
\text { specific geographic areas rather than } \\
\text { pollutant-specific releases associated with } \\
\text { broad national standards. }\end{array}$ \\
\hline $\begin{array}{l}\text { Community Participation } \\
\text { in Superfund Risk } \\
\text { Assessments } \\
\text { (U.S. Environmental } \\
\text { Protection Agency } \\
\text { [EPA]) }\end{array}$ & $\begin{array}{l}\text { Draft reference guide to determine where } \\
\text { community input can augment and improve } \\
\text { estimated exposures and risks from a site. }\end{array}$ & $\begin{array}{l}\text { Guidance ideas are consistent with those } \\
\text { of the RBB. It acknowledges the need for } \\
\text { local community input with regard to } \\
\text { exposures and the cost of obtaining such } \\
\text { input. }\end{array}$ \\
\hline $\begin{array}{l}\text { Community-Based } \\
\text { Environmental } \\
\text { Protection (EPA } \\
\text { Reinvention Office) }\end{array}$ & $\begin{array}{l}\text { To provide a foundation for implementing } \\
\text { community-based environmental protection } \\
\text { that focuses on a definable geographic area; } \\
\text { work collaboratively with a full a range of } \\
\text { stakeholders; assess the quality of air, water, } \\
\text { land, and living resources as a whole; integrate } \\
\text { environmental, economic, and social } \\
\text { objectives; and monitor and redirect efforts } \\
\text { through adaptive management. }\end{array}$ & $\begin{array}{l}\text { Consistent with the RBB approach of } \\
\text { setting risk-based release limits by } \\
\text { considering multimedia impacts and } \\
\text { sources, using participatory process at a } \\
\text { defined geographic level, and providing for } \\
\text { evaluation and modification of program } \\
\text { over time. }\end{array}$ \\
\hline $\begin{array}{l}\text { Core Performance } \\
\text { Measures } \\
\text { (EPA guidance for } \\
\text { evaluating state } \\
\text { environmental } \\
\text { programs) }\end{array}$ & $\begin{array}{l}\text { Developed in conjunction with states to achieve } \\
\text { greater environmental protection, better } \\
\text { measurement of environmental progress, and } \\
\text { the most efficient use of public resources. } \\
\text { Contains three kinds of core performance } \\
\text { measures (environmental outcomes, program } \\
\text { outcomes, and program outputs). }\end{array}$ & $\begin{array}{l}\text { Can provide lessons learned on } \\
\text { developing and using environmental and } \\
\text { program performance indicators for } \\
\text { measuring RBB performance. }\end{array}$ \\
\hline
\end{tabular}


Table F.1 Studies and Efforts That Support the Concepts and Provisions of the RBB (Cont.)

\begin{tabular}{|c|c|c|}
\hline Effort/Organization & Goal/Objective & Congruence with/Support for RBB \\
\hline $\begin{array}{l}\text { Cumulative Risk } \\
\text { Guidelines (EPA) }\end{array}$ & $\begin{array}{l}\text { Major multiyear effort to explore new scientific } \\
\text { and policy realms to account for multimedia, } \\
\text { multipollutant, and multipathway risks. }\end{array}$ & $\begin{array}{l}\text { Can provide lessons learned applicable to } \\
\text { the RBB. }\end{array}$ \\
\hline $\begin{array}{l}\text { Cumulative Risk } \\
\text { Assessment }- \\
\text { Planning and Scoping }\end{array}$ & $\begin{array}{l}\text { To provide a process for considering sources, } \\
\text { pathways, and effects needed to identify and } \\
\text { assess cumulative risks (July 1997). }\end{array}$ & $\begin{array}{l}\text { Formally recognizes the importance of an } \\
\text { agency commitment to addressing the } \\
\text { technical and legal challenges of } \\
\text { considering multiple risks. Information } \\
\text { from this effort will help in implementing } \\
\text { the RBB. }\end{array}$ \\
\hline $\begin{array}{l}\text { Cumulative Air Toxics } \\
\text { Reduction Program } \\
\text { (California South Coast } \\
\text { Air Quality Management } \\
\text { District) }\end{array}$ & $\begin{array}{l}\text { This permit program is designed to limit } \\
\text { cumulative risks from an existing facility to } 100 \\
\text { in } 1,000,000 \text { and from a new facility to } 10 \text { in } \\
1,000,000 \text {. Regulators have discussed } \\
\text { considering the cumulative air quality impact } \\
\text { from nearby facilities when deciding whether to } \\
\text { issue a permit for a new or modified facility. }\end{array}$ & $\begin{array}{l}\text { The implementation of the regulatory } \\
\text { program, which considers cumulative } \\
\text { effects on a local area, provides a } \\
\text { precedent for implementing RBB } \\
\text { provisions that consider cumulative risks. }\end{array}$ \\
\hline $\begin{array}{l}\text { Enlibra (Western } \\
\text { Governors Association) }\end{array}$ & $\begin{array}{l}\text { These environmental policy principles are } \\
\text { designed to emphasize collaborative, flexible } \\
\text { decision making; focus on performance-based } \\
\text { rather than process-based systems; use } \\
\text { objective science and economic incentives; } \\
\text { and recognize environmental differences in } \\
\text { geographic regions. }\end{array}$ & $\begin{array}{l}\text { Its principles of collaborative, flexible } \\
\text { decision making that reflect specific } \\
\text { geographic locations are embodied in the } \\
\text { RBB. }\end{array}$ \\
\hline $\begin{array}{l}\text { Environmental } \\
\text { Monitoring for Public } \\
\text { Access and Community } \\
\text { Tracing (EMPACT) } \\
\text { (EPA) }\end{array}$ & $\begin{array}{l}\text { This } 1996 \text { Presidential initiative is designed to } \\
\text { provide environmental information to the public } \\
\text { in near real time. EMPACT systems reside in, } \\
\text { are managed by, and are maintained by } \\
\text { individual pilot communities. EMPACT uses the } \\
\text { latest technology to track environmental } \\
\text { conditions, present information in plain } \\
\text { language, and ensure that information is } \\
\text { accurate and useful. }\end{array}$ & $\begin{array}{l}\text { The EMPACT handbook can facilitate } \\
\text { RBB collection and distribution of data to } \\
\text { the public. It addresses providing data } \\
\text { through an open access system with } \\
\text { understandable format, data quality } \\
\text { assurance, Web site construction, and } \\
\text { selection of other communication tools. }\end{array}$ \\
\hline $\begin{array}{l}\text { EPA Center for } \\
\text { Environmental Models }\end{array}$ & $\begin{array}{l}\text { In July 1999, the Science Policy Council } \\
\text { approved the formation of a center to guide the } \\
\text { development, calibration, and evaluation of } \\
\text { environmental models. Goals are to ensure } \\
\text { consistency and clarity, account for uncertainty, } \\
\text { provide for peer review, and expand outreach } \\
\text { and public involvement in model development. }\end{array}$ & $\begin{array}{l}\text { A central location of peer-reviewed models } \\
\text { will facilitate the development of the risk- } \\
\text { based modeling system envisioned in the } \\
\text { RBB. }\end{array}$ \\
\hline $\begin{array}{l}\text { GAO Letter on Key } \\
\text { Management Issues } \\
\text { Facing the EPA } \\
\text { (U.S. Government } \\
\text { Accounting Office) }\end{array}$ & $\begin{array}{l}\text { This April } 1998 \text { letter stated that the EPA } \\
\text { needs to improve its performance in } \\
\text { establishing priorities that better reflect risks to } \\
\text { human health and the environment and } \\
\text { consider risks across pollution problems. }\end{array}$ & $\begin{array}{l}\text { Recognizes need to incorporate risk in } \\
\text { regulatory reinvention activities. }\end{array}$ \\
\hline
\end{tabular}


Table F.1 Studies and Efforts That Support the Concepts and Provisions of the RBB (Cont.)

\begin{tabular}{|c|c|c|}
\hline Effort/Organization & Goal/Objective & Congruence with/Support for RBB \\
\hline $\begin{array}{l}\text { Good Neighbor } \\
\text { Initiatives Guidance } \\
\text { (EPA) }\end{array}$ & $\begin{array}{l}\text { To bring together industry, local government, } \\
\text { and community groups in voluntary } \\
\text { partnerships to address environmental issues } \\
\text { in industrialized areas. EPA regions are to } \\
\text { establish such projects by } 2001 .\end{array}$ & $\begin{array}{l}\text { Establishing a good neighbor initiative } \\
\text { could provide a foundation for initiating a } \\
\text { RBB Board and associated public } \\
\text { participation necessary for implementing } \\
\text { the RBB at specific refineries. }\end{array}$ \\
\hline $\begin{array}{l}\text { Government } \\
\text { Performance and } \\
\text { Results Act (GPRA) }\end{array}$ & $\begin{array}{l}\text { To require government agencies to plan their } \\
\text { activities and evaluate the success of their } \\
\text { programs relative to the outcomes of their } \\
\text { actions. }\end{array}$ & $\begin{array}{l}\text { Consistent with inclusion of performance- } \\
\text { based measures in compliance } \\
\text { assurance. }\end{array}$ \\
\hline $\begin{array}{l}\text { Guidance for } \\
\text { Conducting Health Risk } \\
\text { Assessment of } \\
\text { Chemical Mixtures }\end{array}$ & $\begin{array}{l}\text { New guidance (April 1999) designed to update } \\
\text { earlier guidance on assessing risk from } \\
\text { exposure to multiple chemicals and } \\
\text { incorporates state-of-the-art research } \\
\text { methodology. Provides different approaches } \\
\text { depending on type of available data. }\end{array}$ & $\begin{array}{l}\text { Indicates that the EPA and others are } \\
\text { continually developing and incorporating } \\
\text { advances in the areas of chemical } \\
\text { mixtures health risk assessment, which } \\
\text { will help in developing risk-based limits. }\end{array}$ \\
\hline $\begin{array}{l}\text { ISO } 14000 \\
\text { Environmental } \\
\text { Management Standards } \\
\text { ((International } \\
\text { Standards Organization) }\end{array}$ & $\begin{array}{l}\text { To call for continuous improvement in } \\
\text { environmental management and provide for } \\
\text { environmental performance measurement. }\end{array}$ & $\begin{array}{l}\text { Reexamination of limits helps ensure } \\
\text { continuous improvement. RBB } \\
\text { incorporates environmental and program } \\
\text { performance measures }\end{array}$ \\
\hline $\begin{array}{l}\text { EPA/State Agreement } \\
\text { on Regulatory } \\
\text { Innovation } \\
\text { (ECOS) }\end{array}$ & $\begin{array}{l}\text { To give states greater authority to experiment } \\
\text { with innovative approaches to environmental } \\
\text { protection. It establishes principles for the } \\
\text { development, testing, and implementation of } \\
\text { regulatory innovations proposed by states. }\end{array}$ & $\begin{array}{l}\text { Strives to offer flexibility through site- } \\
\text { specific approaches while requiring } \\
\text { accountability. It focuses on integrated } \\
\text { strategies for facilities and communities } \\
\text { rather than on pollutant-by-pollutant } \\
\text { approaches. }\end{array}$ \\
\hline $\begin{array}{l}\text { Minimum Risk Levels } \\
\text { (MRLs) for Hazardous } \\
\text { Substances (ATSDR) }\end{array}$ & $\begin{array}{l}\text { MRLs for } 280 \text { substances are designed to } \\
\text { screen hazardous substances not likely to have } \\
\text { appreciable noncancer health risk. }\end{array}$ & $\begin{array}{l}\text { Supports RBB concept of using risk to } \\
\text { target environmental action. Results could } \\
\text { be used to help set priorities for } \\
\text { establishing limits. }\end{array}$ \\
\hline $\begin{array}{l}\text { National Human } \\
\text { Exposure Assessment } \\
\text { Survey (EPA) }\end{array}$ & $\begin{array}{l}\text { Research effort designed to collect } \\
\text { biomonitoring data and information on } \\
\text { pathways of exposure of high-risk chemicals in } \\
\text { two or more environmental media. }\end{array}$ & $\begin{array}{l}\text { Real-life exposure data from multiple } \\
\text { substances and exposure routes will } \\
\text { improve and accelerate development of } \\
\text { risk-based limits. }\end{array}$ \\
\hline $\begin{array}{l}\text { National Academy of } \\
\text { Engineering Report on } \\
\text { Environmental } \\
\text { Performance Metrics }\end{array}$ & $\begin{array}{l}\text { To call for the U.S. government to lead an } \\
\text { effort to develop standardized yardsticks for } \\
\text { measuring industrial environmental } \\
\text { performance. It recommends that companies } \\
\text { and industry sectors set quantitative } \\
\text { environmental goals and then track and report } \\
\text { progress in meeting those goals. }\end{array}$ & $\begin{array}{l}\text { Is consistent with the RBB requirement to } \\
\text { account for and measure environmental } \\
\text { progress, given the additional flexibility in } \\
\text { meeting environmental goals. }\end{array}$ \\
\hline
\end{tabular}


Table F.1 Studies and Efforts That Support the Concepts and Provisions of the RBB (Cont.)

\begin{tabular}{|c|c|c|}
\hline Effort/Organization & Goal/Objective & Congruence with/Support for RBB \\
\hline $\begin{array}{l}\text { Performance-Based } \\
\text { Measurement System } \\
\text { (PBMS) (EPA) }\end{array}$ & $\begin{array}{l}\text { To allow facilities to use alternative } \\
\text { measurement systems that reflect site-specific } \\
\text { needs as long as the alternatives meet EPA- } \\
\text { established performance criteria that address } \\
\text { acceptable levels of uncertainty, performance } \\
\text { verification, etc. }\end{array}$ & $\begin{array}{l}\text { Such site-specific flexibility in } \\
\text { measurement systems provides a } \\
\text { precedent for allowing flexibility in broader } \\
\text { environmental compliance technologies } \\
\text { and other alternative approaches. }\end{array}$ \\
\hline $\begin{array}{l}\text { Pilot Projects to } \\
\text { Measure Effectiveness } \\
\text { of Compliance } \\
\text { Assurance Programs }\end{array}$ & $\begin{array}{l}\text { Part of the EPA's effort to move from traditional } \\
\text { enforcement metrics (e.g., number of } \\
\text { enforcement actions) to outcome-based } \\
\text { metrics that measure the effect of compliance } \\
\text { on human health and the environment. }\end{array}$ & $\begin{array}{l}\text { May provide lessons learned for } \\
\text { measuring success of RBB approach. }\end{array}$ \\
\hline $\begin{array}{l}\text { Presidential/ } \\
\text { Congressional } \\
\text { Commission on Risk } \\
\text { Assessment and Risk } \\
\text { Management in } \\
\text { Regulatory Decision } \\
\text { Making }\end{array}$ & $\begin{array}{l}\text { To investigate the appropriate uses of risk } \\
\text { management in regulatory programs to prevent } \\
\text { human health effects that may result from } \\
\text { exposure to hazardous substances. It proposes } \\
\text { a framework for risk management that would } \\
\text { evaluate total exposure to substances from all } \\
\text { sources in all media, rather than single-risk } \\
\text { exposures from a single medium. }\end{array}$ & $\begin{array}{l}\text { Suggests a framework similar to the RBB } \\
\text { (i.e., identifies/characterizes environmental } \\
\text { problems, puts environmental problems } \\
\text { into context, determines risk management } \\
\text { goals, engages stakeholders). It } \\
\text { advocates and provides framework for } \\
\text { assessing total cumulative risk. Its } \\
\text { recommendations (e.g., requiring } \\
\text { Congress to consider legislative changes } \\
\text { that increase flexibility for meeting } \\
\text { environmental protection goals) could } \\
\text { facilitate RBB implementation. }\end{array}$ \\
\hline $\begin{array}{l}\text { Proposal to Require } \\
\text { Data on Exposure for } \\
\text { Manufactured or } \\
\text { Imported Chemicals } \\
\text { (EPA) }\end{array}$ & $\begin{array}{l}\text { The August } 1999 \text { proposal would require } \\
\text { companies that manufacture or import } \\
\text { chemicals to submit exposure data for the } \\
\text { EPA's Chemical Substances Inventory. Data } \\
\text { would include number of ecosystems or size of } \\
\text { human population exposed; potential exposure } \\
\text { routes; magnitudes, concentrations, } \\
\text { frequencies, and durations of potential } \\
\text { exposures. }\end{array}$ & $\begin{array}{l}\text { A centralized source of chemical exposure } \\
\text { data will help in setting priorities and } \\
\text { developing risk-based release limits. }\end{array}$ \\
\hline $\begin{array}{l}\text { Proposed Economic } \\
\text { Incentive Program } \\
\text { Guidance (EPA Office of } \\
\text { Air and Radiation) }\end{array}$ & $\begin{array}{l}\text { To provide guidance for using market-based } \\
\text { strategies to provide compliance flexibility, } \\
\text { encourage technology innovation, and reduce } \\
\text { emissions of air pollutants in the most efficient } \\
\text { manner. Examples include source-specific } \\
\text { emissions caps and multisource emission cap- } \\
\text { and-trade programs. }\end{array}$ & $\begin{array}{l}\text { The guidance provides for cross-pollutant } \\
\text { trading (e.g., trade nitrogen oxides } \\
\text { emissions for volatile organic compound } \\
\text { emissions). The exchange of releases } \\
\text { among various pollutants and media, as } \\
\text { long as total cumulative risk levels are } \\
\text { acceptable, is a key provision of the RBB. }\end{array}$ \\
\hline $\begin{array}{l}\text { Quantitative } \\
\text { Environmental } \\
\text { Indicators of } \\
\text { Contamination (New } \\
\text { Jersey Department of } \\
\text { Environmental } \\
\text { Protection) }\end{array}$ & $\begin{array}{l}\text { To use a set of basic scientific measures, } \\
\text { coupled with geographic information system } \\
\text { techniques, to summarize the quality of soil } \\
\text { and groundwater and the impact of } \\
\text { groundwater contamination on surface water at } \\
\text { contaminated sites in communities. }\end{array}$ & $\begin{array}{l}\text { Provides examples of the kinds of } \\
\text { reporting techniques that can be used to } \\
\text { share cumulative environmental } \\
\text { information with the public and regulators. }\end{array}$ \\
\hline
\end{tabular}


Table F.1 Studies and Efforts That Support the Concepts and Provisions of the RBB (Cont.)

\begin{tabular}{|c|c|c|}
\hline Effort/Organization & Goal/Objective & Congruence with/Support for RBB \\
\hline $\begin{array}{l}\text { Residual Risk Report to } \\
\text { Congress (OAQPS) }\end{array}$ & $\begin{array}{l}\text { To describe the methods and framework that } \\
\text { the EPA will use to assess public health and } \\
\text { the environmental risk that may remain after } \\
\text { implementation of maximum achievable control } \\
\text { technology (MACT) regulations. It provides } \\
\text { background on risk assessment techniques, } \\
\text { data, and the current state of knowledge. }\end{array}$ & $\begin{array}{l}\text { Comprehensive and current (March 1999) } \\
\text { source of information on data, data } \\
\text { sources, risk assessment methodologies, } \\
\text { current state of knowledge, etc. It provides } \\
\text { the foundation needed to begin developing } \\
\text { a process for establishing risk-based } \\
\text { limits. }\end{array}$ \\
\hline $\begin{array}{l}\text { Risk Screening } \\
\text { Environmental } \\
\text { Indicators (RSEIs) } \\
\text { (EPA Office of } \\
\text { Prevention, Pesticides } \\
\text { and Toxic Substances } \\
\text { [OPPT]) }\end{array}$ & $\begin{array}{l}\text { RSEls combine data on quantities of air } \\
\text { pollutants released, toxicity, populations } \\
\text { exposed, and dose to generate chronic human } \\
\text { health indicators for more than } 500 \text { chemicals } \\
\text { and } 38,000 \text { sites. The indicators provide a } \\
\text { screening-level, risk-related perspective for } \\
\text { comparing chemical releases and can help } \\
\text { rank and prioritize chemicals for risk-related } \\
\text { targeting and community-based environmental } \\
\text { protection. OPPT plans to develop indicators } \\
\text { for acute human health impacts and chronic } \\
\text { and acute ecological impacts. }\end{array}$ & $\begin{array}{l}\text { RSEls could be used to establish priorities } \\
\text { for setting the risk-based limits. In } \\
\text { addition, the RSEls embody a vast } \\
\text { amount of data that could be used to } \\
\text { establish risk-based limits. The existence } \\
\text { of this newly available system (released by } \\
\text { the EPA in July } 1999 \text { and under } \\
\text { development for } 10 \text { years) and its data will } \\
\text { hasten the ability to develop risk-based } \\
\text { limits. }\end{array}$ \\
\hline $\begin{array}{l}\text { Science Advisory Board } \\
\text { (SAB) Integrated Risk } \\
\text { Project (IRP) }\end{array}$ & $\begin{array}{l}\text { An SAB draft report suggests a framework for } \\
\text { environmental decision making integrate } \\
\text { scientific information, comparative risk, } \\
\text { economic considerations, impact of public } \\
\text { values, and a range of management options. } \\
\text { It recommends focusing on reducing total } \\
\text { aggregate risk rather than reducing the worst } \\
\text { risks. }\end{array}$ & $\begin{array}{l}\text { The SAB draft report recommendation that } \\
\text { the EPA shift from a chemical-by-chemical } \\
\text { approach to an integrated process that } \\
\text { considers total aggregate risk for meeting } \\
\text { environmental protection goals is } \\
\text { consistent with the RBB. Similarly, the } \\
\text { need to incorporate public opinion and } \\
\text { performance measures is reflected in the } \\
\text { RBB. }\end{array}$ \\
\hline $\begin{array}{l}\text { Sector Facility Indexing } \\
\text { Project (SFIP) (EPA } \\
\text { Office of Enforcement } \\
\text { and Compliance } \\
\text { Assistance) }\end{array}$ & $\begin{array}{l}\text { To provide greater public access to compliance } \\
\text { and facility-level information for five sectors, } \\
\text { one of which is petroleum refining. It currently } \\
\text { includes compliance, enforcement, chemical } \\
\text { release, production, and demographic data. } \\
\text { The EPA is working to include an indicator that } \\
\text { combines Toxic Release Inventory (TRI) data } \\
\text { with toxicity data to develop an index that } \\
\text { considers chronic human health hazards. }\end{array}$ & $\begin{array}{l}\text { Supports RBB concept of considering not } \\
\text { only the quantities of emissions but also } \\
\text { their toxicity. }\end{array}$ \\
\hline $\begin{array}{l}\text { EPA/State Long-Term } \\
\text { Air Monitoring Strategy }\end{array}$ & $\begin{array}{l}\text { Effort is in early stages. The concept is to } \\
\text { develop a long-term strategy to monitor all air } \\
\text { contaminants over a period of } 10 \text { years or } \\
\text { longer. }\end{array}$ & $\begin{array}{l}\text { The ability to use the results of existing } \\
\text { monitoring efforts would facilitate RBB } \\
\text { implementation. }\end{array}$ \\
\hline
\end{tabular}


A 


\section{Appendix G Summary of Approaches to Legislative Change}

Table G.1 Summary of Approaches to Legislative Change

\begin{tabular}{|c|c|c|c|}
\hline \multirow[b]{2}{*}{ Parameter } & \multicolumn{3}{|c|}{ Approach } \\
\hline & Pilot-Study Waiver & Strategic Waiver & $\begin{array}{c}\text { Individual Statutory } \\
\text { Change }\end{array}$ \\
\hline $\begin{array}{l}\text { Form of legislative } \\
\text { action }\end{array}$ & $\begin{array}{l}\text { Amendment to } \\
\text { appropriations bill }\end{array}$ & Separate bill & $\begin{array}{l}\text { Amendments to individual } \\
\text { statutes }\end{array}$ \\
\hline Precedent/example & $\begin{array}{l}\text { Provisions for expediting } \\
\text { cleanup at a particular } \\
\text { Superfund site }\end{array}$ & $\begin{array}{l}\text { S. } 1348, \text { The Innovative } \\
\text { Environmental Strategies Act } \\
\text { of } 1997 \text {, introduced by Sen. } \\
\text { Joseph Lieberman in October } \\
1997 \\
\text { - Accountable Pipeline Safety } \\
\text { and Partnership Act of } 1996\end{array}$ & $\begin{array}{l}\text { - Resource Conservation and } \\
\text { Recovery Act (RCRA) reform } \\
\text { proposals } \\
\text { - Safe Drinking Water Act } \\
\text { (SDQA) Amendments of } \\
1996\end{array}$ \\
\hline Advantages & $\begin{array}{l}\text { Relatively good chance } \\
\text { for passage; relatively } \\
\text { simple to draft }\end{array}$ & $\begin{array}{l}\text { Could be applied to other } \\
\text { industries and could be used } \\
\text { beyond pilot studies }\end{array}$ & $\begin{array}{l}\text { Could result in permanent } \\
\text { changes that would provide } \\
\text { more flexibility for more } \\
\text { industries }\end{array}$ \\
\hline Disadvantages & $\begin{array}{l}\text { Illicits concern from } \\
\text { regulators and } \\
\text { environmental groups } \\
\text { that the approach could } \\
\text { set a precedent }\end{array}$ & $\begin{array}{l}\text { May be difficult to pass because } \\
\text { it is a stand-alone measure }\end{array}$ & $\begin{array}{l}\text { Requires a lot of time to } \\
\text { develop line-by-line language to } \\
\text { address all possible sections of } \\
\text { all possible legislation }\end{array}$ \\
\hline
\end{tabular}


A 


\title{
Appendix $\mathrm{H}$ Studies and Efforts That Support the Concepts and Provisions of the Negotiated Performance Agreement
}

\author{
Table H.1 Studies and Efforts That Support the Concepts and Provisions of the \\ Negotiated Performance Agreement (NPA)
}

\begin{tabular}{|c|c|c|}
\hline Effort/Organization & Goal/Objective & Congruence with/Support for NPA \\
\hline $\begin{array}{l}\text { National Commission } \\
\text { on Environment }\end{array}$ & $\begin{array}{l}\text { To promote legislation that provides for the } \\
\text { issuance of multimedia permits that cover all } \\
\text { forms of pollution from all sources within a } \\
\text { facility. }\end{array}$ & $\begin{array}{l}\text { Multimedia permitting is the core of the NPA; } \\
\text { legislation requiring such permitting would } \\
\text { facilitate implementation. }\end{array}$ \\
\hline $\begin{array}{l}\text { Air Information } \\
\text { Reporting System } \\
\text { (AIRS) for Refineries }\end{array}$ & $\begin{array}{l}\text { Formerly known as the One Stop Reporting } \\
\text { and Public Access Project, the goal of this } \\
\text { Common Sense Initiative project of the } \\
\text { petroleum refining sector is to identify and } \\
\text { recommend modifications to existing air } \\
\text { reporting requirements that are duplicative } \\
\text { and/or obsolete and to address community } \\
\text { needs for increased understanding of and } \\
\text { access to reported environmental } \\
\text { information. }\end{array}$ & $\begin{array}{l}\text { Recognizes the need to provide for } \\
\text { streamlined reporting and to increase public } \\
\text { access and understanding of environmental } \\
\text { reports. The initial phases were expected to } \\
\text { lead to the development of a multimedia pilot } \\
\text { of regulatory reporting requirements, which } \\
\text { could be directly applicable to the NPA. }\end{array}$ \\
\hline $\begin{array}{l}\text { Aspen Institute's } \\
\text { Alternative Path }\end{array}$ & $\begin{array}{l}\text { To develop a new environmental } \\
\text { management system through dialogue with } \\
\text { leaders of business, government, } \\
\text { environmental, and other public interest } \\
\text { groups. }\end{array}$ & $\begin{array}{l}\text { Consistent with the NPA approach of } \\
\text { allowing companies to design a more } \\
\text { efficient environmental management plan } \\
\text { with increased flexibility in return for } \\
\text { developing that plan in an open, transparent, } \\
\text { stakeholder process. }\end{array}$ \\
\hline $\begin{array}{l}\text { Enterprise for the } \\
\text { Environment }\end{array}$ & $\begin{array}{l}\text { To recommend changes to the nation's } \\
\text { environmental protection system so it } \\
\text { supports collaboration in decision making } \\
\text { (so all interests are heard) and encourages } \\
\text { facilitywide, multimedia approaches for } \\
\text { identifying pollution prevention opportunities. }\end{array}$ & $\begin{array}{l}\text { These objectives are central to the NPA } \\
\text { approach of using multimedia permitting to } \\
\text { meet environmental protection goals that } \\
\text { incorporate pollution prevention. }\end{array}$ \\
\hline $\begin{array}{l}\text { Project XL } \\
\text { (U.S. Environmental } \\
\text { Protection Agency } \\
\text { [EPA]) }\end{array}$ & $\begin{array}{l}\text { To exchange increased flexibility and } \\
\text { regulatory relief for superior environmental } \\
\text { performance, on a project-specific basis. } \\
\text { Stakeholder involvement is a key } \\
\text { component of meeting these goals. }\end{array}$ & $\begin{array}{l}\text { Consistent with the NPA approach of using } \\
\text { collaborative working relationship with } \\
\text { industries and people who believe their } \\
\text { community could be affected by the project. }\end{array}$ \\
\hline $\begin{array}{l}\text { Netherlands Covenant } \\
\text { Program }\end{array}$ & $\begin{array}{l}\text { To change the nation's environmental } \\
\text { protection system through the use of } \\
\text { negotiated covenants, which set specific } \\
\text { environmental targets, linked to overall } \\
\text { national objectives. Such targets are to be } \\
\text { met according to an agreed-upon schedule. }\end{array}$ & $\begin{array}{l}\text { Embodies the concept of negotiating an } \\
\text { agreement with the regulators and the } \\
\text { regulated entities, which is key to the NPA. It } \\
\text { also uses stakeholder input and provides } \\
\text { penalties for noncompliance. }\end{array}$ \\
\hline
\end{tabular}

Continued 
Table H.1 Studies and Efforts That Support the Concepts and Provisions of the NPA (Cont.)

\begin{tabular}{|c|c|c|}
\hline Effort/Organization & Goal/Objective & Congruence with/Support for NPA \\
\hline $\begin{array}{l}\text { New Jersey Facility- } \\
\text { Wide Permit Program }\end{array}$ & $\begin{array}{l}\text { To improve environmental quality via } \\
\text { multimedia emissions reduction, maximize } \\
\text { opportunities for pollution prevention, } \\
\text { provide greater operational flexibility, and } \\
\text { streamline regulatory requirements. }\end{array}$ & $\begin{array}{l}\text { Provides a working model for multimedia, } \\
\text { facilitywide permits. It also provides } \\
\text { approaches for identifying residuals for } \\
\text { baselines and for setting limits. Results of } \\
\text { the program will provide lessons learned for } \\
\text { implementing the NPA. }\end{array}$ \\
\hline $\begin{array}{l}\text { EPA Environmental } \\
\text { Permits Improvement } \\
\text { Team } \\
\text { Recommendations }\end{array}$ & $\begin{array}{l}\text { To improve environmental permitting } \\
\text { processes by using public performance- } \\
\text { based permitting. It is based on the principle } \\
\text { that public access to information can lead to } \\
\text { improved environmental results with less } \\
\text { government involvement in facility activities. } \\
\text { It shifts the focus of permitting toward } \\
\text { measurement and assurance of a defined } \\
\text { level of performance, while providing } \\
\text { flexibility as to how a permittee will meet } \\
\text { performance standards. }\end{array}$ & $\begin{array}{l}\text { The NPA embodies the concept of increased } \\
\text { flexibility along with increased accountability } \\
\text { via public access to reports. It is thus } \\
\text { consistent with the recommendations of the } \\
\text { Environmental Improvement Permits Team. }\end{array}$ \\
\hline $\begin{array}{l}\text { National Advisory } \\
\text { Council for } \\
\text { Environmental Policy } \\
\text { and Technology } \\
\text { (NACEPT), Technology } \\
\text { Innovation and } \\
\text { Economics Committee }\end{array}$ & $\begin{array}{l}\text { To investigate the use of permitting and } \\
\text { compliance polices to foster pollution } \\
\text { prevention in the context of industrial } \\
\text { production and manufacturing. }\end{array}$ & $\begin{array}{l}\text { Multimedia permitting systems could } \\
\text { facilitate the introduction and use of pollution } \\
\text { prevention technologies and practices } \\
\text { throughout U.S. industry. }\end{array}$ \\
\hline $\begin{array}{l}\text { National Pollution } \\
\text { Prevention Roundtable }\end{array}$ & $\begin{array}{l}\text { To promote pollution prevention strategies } \\
\text { and seek bipartisan Congressional support } \\
\text { to reauthorize the Pollution Prevention Act. It } \\
\text { recommends that states develop and } \\
\text { implement multimedia pollution prevention } \\
\text { planning programs for facilities and that } \\
\text { current funding levels for pollution } \\
\text { prevention programs be increased. }\end{array}$ & $\begin{array}{l}\text { Its recommendations are consistent with the } \\
\text { NPA's principle of fostering pollution } \\
\text { prevention as a means of meeting } \\
\text { environmental objectives. It recognizes the } \\
\text { need for financial and legislative support to } \\
\text { facilitate use of pollution prevention. }\end{array}$ \\
\hline $\begin{array}{l}\text { National Air Quality } \\
\text { Operating Committee }\end{array}$ & $\begin{array}{l}\text { The EPA Air Office Suggested that this } \\
\text { committee be formed to adopt the long- } \\
\text { range view of EPA's Air Program. }\end{array}$ & $\begin{array}{l}\text { The goal of seeking cooperative strategies to } \\
\text { identify long-range goals is consistent with } \\
\text { the NPA's long-range approach. }\end{array}$ \\
\hline $\begin{array}{l}\text { Action Plan for } \\
\text { Innovation (EPA) }\end{array}$ & $\begin{array}{l}\text { To use stakeholder outreach to improve } \\
\text { enforcement and compliance. It } \\
\text { recommends involving communities in } \\
\text { developing supplemental environmental } \\
\text { projects and publicizing innovative } \\
\text { supplemental environmental projects } \\
\text { (SEPs). }\end{array}$ & $\begin{array}{l}\text { Concept is consistent with the NPA's } \\
\text { compliance assurance concept of } \\
\text { compensating local communities for refinery } \\
\text { violations of release limits. }\end{array}$ \\
\hline
\end{tabular}


Table H.1 Studies and Efforts That Support the Concepts and Provisions of the NPA (Cont.)

\begin{tabular}{|c|c|c|}
\hline Effort/Organization & Goal/Objective & Congruence with/Support for NPA \\
\hline $\begin{array}{l}\text { Reinventing } \\
\text { Environmental } \\
\text { Regulations - National } \\
\text { Performance Review }\end{array}$ & $\begin{array}{l}\text { In this } 1995 \text { report, often cited as the basis } \\
\text { for reinventing environmental regulations, } \\
\text { the President and Vice President identified } \\
25 \text { high-priority actions to improve the } \\
\text { existing regulatory system and move toward } \\
\text { a better environmental management system } \\
\text { in the } 21^{\text {st }} \text { century. The report found that } \\
\text { using performance standards with economic } \\
\text { incentives encourages innovation and that } \\
\text { increased flexibility requires increased } \\
\text { accountability and responsibility. }\end{array}$ & $\begin{array}{l}\text { The report's recommendations to increase } \\
\text { the use of (1) regulatory negotiation, } \\
\text { (2) electronic communication of } \\
\text { environmental information, and } \\
\text { (3) multimedia, consolidated reporting are all } \\
\text { consistent with and embodied in the NPA. }\end{array}$ \\
\hline $\begin{array}{l}\text { EPA Report on } \\
\text { Reinventing } \\
\text { Environmental } \\
\text { Protection (Office of the } \\
\text { Administrator) }\end{array}$ & $\begin{array}{l}\text { To provide progress report on EPA } \\
\text { approaches to reinventing environmental } \\
\text { regulation. It notes the role of the } \\
\text { Government Performance and Results Act } \\
\text { of } 1993 \text { in setting national environmental } \\
\text { goals. }\end{array}$ & $\begin{array}{l}\text { National-level goals can help focus the goals } \\
\text { and objectives of specific NPAs. }\end{array}$ \\
\hline $\begin{array}{l}\text { Community-Based } \\
\text { Environmental } \\
\text { Protection (EPA } \\
\text { Reinvention Office) }\end{array}$ & $\begin{array}{l}\text { To provide a foundation for implementing } \\
\text { community-based environmental protection } \\
\text { that focuses on a definable geographic area. } \\
\text { It promotes collaboration with a full range of } \\
\text { stakeholders. }\end{array}$ & $\begin{array}{l}\text { Consistent with the NPA approach of } \\
\text { considering multimedia impacts and } \\
\text { sources, using the participatory process at a } \\
\text { defined geographic level, and providing for } \\
\text { evaluation and modification of the program } \\
\text { over time. }\end{array}$ \\
\hline $\begin{array}{l}\text { Core Performance } \\
\text { Measures } \\
\text { (EPA guidance for } \\
\text { evaluating state } \\
\text { environmental } \\
\text { programs) }\end{array}$ & $\begin{array}{l}\text { Developed in conjunction with states to } \\
\text { achieve greater environmental protection, } \\
\text { better measurements of environmental } \\
\text { progress, and the most efficient use of } \\
\text { public resources. Contains three kinds of } \\
\text { core performance measures (environmental } \\
\text { outcomes, program outcomes, and program } \\
\text { outputs). }\end{array}$ & $\begin{array}{l}\text { Can provide lessons learned on developing } \\
\text { and using environmental and program } \\
\text { performance indicators for measuring NPA } \\
\text { performance. }\end{array}$ \\
\hline $\begin{array}{l}\text { Enlibra (Western } \\
\text { Governors Association) }\end{array}$ & $\begin{array}{l}\text { These environmental policy principles are } \\
\text { designed to emphasize collaborative, } \\
\text { flexible decision making; focus on } \\
\text { performance-based rather than process- } \\
\text { based systems; use objective science and } \\
\text { economic initiatives; and recognize } \\
\text { environmental differences in geographic } \\
\text { regions. }\end{array}$ & $\begin{array}{l}\text { Principles of collaborative, flexible decision } \\
\text { making that reflect specific geographic } \\
\text { locations are embodied in the NPA. }\end{array}$ \\
\hline $\begin{array}{l}\text { Environmental } \\
\text { Monitoring for Public } \\
\text { Access and Community } \\
\text { Tracing (EMPACT) } \\
\text { (EPA) }\end{array}$ & $\begin{array}{l}\text { This } 1996 \text { Presidential initiative is designed } \\
\text { to provide environmental information to the } \\
\text { public in near real time. EMPACT systems } \\
\text { reside in, are managed by, and are } \\
\text { maintained by individual pilot communities. } \\
\text { EMPACT uses the latest technology to track } \\
\text { environmental conditions, present } \\
\text { information in plain language, and ensure } \\
\text { that information is accurate and useful. }\end{array}$ & $\begin{array}{l}\text { The EMPACT handbook can facilitate NPA } \\
\text { collection and distribution of data to the } \\
\text { public. It addresses providing data through } \\
\text { an open access system with understandable } \\
\text { format. }\end{array}$ \\
\hline
\end{tabular}


Table H.1 Studies and Efforts That Support the Concepts and Provisions of the NPA (Cont.)

\begin{tabular}{|c|c|c|}
\hline Effort/Organization & Goal/Objective & Congruence with/Support for NPA \\
\hline $\begin{array}{l}\text { Good Neighbor } \\
\text { Initiatives Guidance } \\
\text { (EPA) }\end{array}$ & $\begin{array}{l}\text { To bring together industry, local } \\
\text { government, and community groups in } \\
\text { voluntary partnerships to address } \\
\text { environmental issues in industrialized areas. } \\
\text { EPA regions are to establish such projects } \\
\text { by } 2001 .\end{array}$ & $\begin{array}{l}\text { Establishing a good neighbor initiative could } \\
\text { provide a foundation for initiating an NPA } \\
\text { Council and associated public participation } \\
\text { necessary for implementing the NPA at } \\
\text { specific refineries. }\end{array}$ \\
\hline $\begin{array}{l}\text { Government } \\
\text { Performance and } \\
\text { Results Act (GPRA) }\end{array}$ & $\begin{array}{l}\text { To require government agencies to plan } \\
\text { their activities and evaluate the success of } \\
\text { their programs relative to the outcomes of } \\
\text { their actions. }\end{array}$ & $\begin{array}{l}\text { Consistent with inclusion of performance- } \\
\text { based measures in compliance assurance. }\end{array}$ \\
\hline $\begin{array}{l}\text { EPA/State Agreement } \\
\text { on Regulatory } \\
\text { Innovation } \\
\text { (ECOS) }\end{array}$ & $\begin{array}{l}\text { To give states greater authority to } \\
\text { experiment with innovative approaches to } \\
\text { environmental protection. It establishes } \\
\text { principles for the development, testing, and } \\
\text { implementation of regulatory innovations } \\
\text { proposed by states. }\end{array}$ & $\begin{array}{l}\text { Strives to offer flexibility through site-specific } \\
\text { approaches while requiring accountability, } \\
\text { which is consistent with the revised NPA. }\end{array}$ \\
\hline $\begin{array}{l}\text { National Academy of } \\
\text { Engineering Report on } \\
\text { Environmental } \\
\text { Performance Metrics }\end{array}$ & $\begin{array}{l}\text { To call for the U.S. government to lead an } \\
\text { effort to develop standardized yardsticks for } \\
\text { measuring industrial environmental } \\
\text { performance. It recommends that } \\
\text { companies and industry sectors set } \\
\text { quantitative environmental goals and then } \\
\text { track and report progress in meeting those } \\
\text { goals. }\end{array}$ & $\begin{array}{l}\text { Consistent with the NPA requirement to } \\
\text { account for and measure environmental } \\
\text { progress, given the additional flexibility in } \\
\text { meeting environmental goals. }\end{array}$ \\
\hline $\begin{array}{l}\text { Performance-Based } \\
\text { Measurement System } \\
\text { (PBMS) (EPA) }\end{array}$ & $\begin{array}{l}\text { To allow facilities to use alternative } \\
\text { measurement systems that reflect site- } \\
\text { specific needs as long as the alternatives } \\
\text { meet EPA-established performance criteria } \\
\text { that address acceptable levels of } \\
\text { uncertainty, performance verification, etc. }\end{array}$ & $\begin{array}{l}\text { Such site-specific flexibility in measurement } \\
\text { systems provides a precedent for allowing } \\
\text { flexibility in broader environmental } \\
\text { compliance technologies and other } \\
\text { alternative approaches. }\end{array}$ \\
\hline $\begin{array}{l}\text { EPA/State Long-Term } \\
\text { Air Monitoring Strategy }\end{array}$ & $\begin{array}{l}\text { Effort is in early stages. The concept is to } \\
\text { develop a long-term strategy to monitor all } \\
\text { air contaminants over a period of } 10 \text { years } \\
\text { or longer. }\end{array}$ & $\begin{array}{l}\text { The ability to use the results of existing } \\
\text { monitoring efforts would facilitate NPA } \\
\text { implementation. }\end{array}$ \\
\hline
\end{tabular}




\section{Appendix I XL Projects Relevant to the Revised Negotiated Performance Agreement}

Table I.1 XL Projects Relevant to the Revised Negotiated Performance Agreement (NPA)

\begin{tabular}{|c|c|c|c|}
\hline XL Project & $\begin{array}{c}\text { Components Similar } \\
\text { to NPA }\end{array}$ & Benefits/Regulatory Relief & $\begin{array}{c}\text { Lessons Applicable } \\
\text { to NPA }\end{array}$ \\
\hline $\begin{array}{l}\text { Merck \& Co., } \\
\text { Stonewall Plant, } \\
\text { Elkton, VA }\end{array}$ & $\begin{array}{l}\text { - Goal-based limits } \\
\text { Facilitywide air pollution cap: } \\
\text { Emissions of } \mathrm{SO}_{2} \text { and } \mathrm{NO}_{x} \text { to } \\
\text { remain at least } 20 \% \text { below } \\
1992 \text { and } 1993 \text { levels } \\
\text { - Incentives: Reporting and } \\
\text { recordkeeping requirements } \\
\text { become more stringent as } \\
\text { emissions approach } \\
\text { facilitywide cap }\end{array}$ & $\begin{array}{l}\text { Eliminates permit reviews for } \\
\text { every process change } \\
\text { - Can reduce facilitywide caps } \\
\text { instead of implementing } \\
\text { specific control technologies } \\
\text { that could be required by } \\
\text { future regulations }\end{array}$ & $\begin{array}{l}\text { - Community stakeholders } \\
\text { need technical support } \\
\text { early in the process } \\
\text { - Third-party facilitation can } \\
\text { help negotiating processes } \\
\text { - Transaction costs for } \\
\text { stakeholders are high }\end{array}$ \\
\hline $\begin{array}{l}\text { Weyerhaeuser, } \\
\text { Flint River } \\
\text { Operations, } \\
\text { Pulp Manufact- } \\
\text { uring } \\
\text { Operations, } \\
\text { Oglethorpe, GA }\end{array}$ & $\begin{array}{l}\text { Facilitywide permit to reduce } \\
\text { wastewater discharges, air } \\
\text { emissions, and solid waste } \\
\text { generation } \\
\text { - Goal-based emissions } \\
\text { reductions: Allowable air } \\
\text { emissions to be reduced by } \\
60 \% \text { by using two emissions } \\
\text { caps - one for the plant's four } \\
\text { major sources and the other for } \\
\text { the remaining sources. } \\
\text { - Consolidated reporting: } \\
\text { Federal, state, and local air } \\
\text { and water reports consolidated } \\
\text { into two reports per year }\end{array}$ & $\begin{array}{l}\text { - Allows process modifications } \\
\text { to be made without obtaining } \\
\text { prior approval } \\
\text { - Streamlines wastewater } \\
\text { permit renewal process } \\
\text { - Eliminates unnecessary } \\
\text { sampling } \\
\text { - Replaces monthly reporting } \\
\text { with annual certification } \\
\text { - Avoids } \$ 10 \text { million in future } \\
\text { capital spending and saves } \\
\$ 200,000 / \text { year from solid } \\
\text { waste recycling }\end{array}$ & $\begin{array}{l}\text { - Site visits build trust and } \\
\text { educate regulators } \\
\text { - Stakeholders need } \\
\text { technical assistance early } \\
\text { in the process } \\
\text { - Using clear language in } \\
\text { agreement facilitates } \\
\text { implementation } \\
\text { - Studies on changes to } \\
\text { manufacturing processes } \\
\text { take time }\end{array}$ \\
\hline $\begin{array}{l}\text { Vandenberg Air } \\
\text { Force Base, } \\
\text { Santa Barbara } \\
\text { County, CA }\end{array}$ & $\begin{array}{l}\text { Agreed-upon, goal-based } \\
\text { limits: Reduction in annual } \\
\text { emissions of ozone-causing } \\
\text { chemicals by } 10 \text { tons or more } \\
\text { by } 2002 \\
\text { - Program details specified in } \\
\text { enforceable emissions } \\
\text { reduction plan prepared by the } \\
\text { facility }\end{array}$ & $\begin{array}{l}\text { - Achieves less administrative } \\
\text { burden with new method of } \\
\text { grouping activities; resources } \\
\text { saved will be used to reduce } \\
\text { emissions } \\
\text { - Incorporates negotiated } \\
\text { protocol for source testing } \\
\text { and validation; allows facility } \\
\text { to use tests that cost } \$ 600 \\
\text { rather than the EPA's test, } \\
\text { which costs } \$ 3,000\end{array}$ & $\begin{array}{l}\text { The potential for citizen } \\
\text { lawsuits creates anxiety } \\
\text { among those who would } \\
\text { otherwise be willing to try } \\
\text { innovative approaches } \\
\text { - The involvement of too } \\
\text { many people slows } \\
\text { negotiations } \\
\text { - Research and } \\
\text { development are resource- } \\
\text { intensive } \\
\text { - An agreement that } \\
\text { provides flexibility during } \\
\text { implementation helps } \\
\text { overcome obstacles }\end{array}$ \\
\hline
\end{tabular}

Continued 
Table I.1 XL Projects Relevant to the Revised NPA (Cont.)

\begin{tabular}{|c|c|c|c|}
\hline XL Project & $\begin{array}{c}\text { Components Similar } \\
\text { to NPA }\end{array}$ & Benefits/Regulatory Relief & $\begin{array}{c}\text { Lessons Applicable } \\
\text { to NPA }\end{array}$ \\
\hline $\begin{array}{l}\text { Massachusetts } \\
\text { Environmental } \\
\text { Results Program }\end{array}$ & $\begin{array}{l}\text { - Facilitywide, performance- } \\
\text { based, self-certification } \\
\text { program } \\
\text { - Pollution prevention to be used } \\
\text { to improve environmental } \\
\text { performance }\end{array}$ & $\begin{array}{l}\text { - Streamlines permitting and } \\
\text { reporting requirements }\end{array}$ & $\begin{array}{l}\text { - Clear performance } \\
\text { standards written in plain } \\
\text { English facilitate } \\
\text { implementation }\end{array}$ \\
\hline $\begin{array}{l}\text { Intel } \\
\text { Semiconductor } \\
\text { Manufacturing } \\
\text { Facility, } \\
\text { Chandler, AZ }\end{array}$ & $\begin{array}{l}\text { - Facilitywide pollution cap } \\
\text { - Consolidated reporting: } \\
\text { - Stakeholder input to } \\
\text { redesign reports } \\
\text { - Environmental results } \\
\text { published on Internet }\end{array}$ & $\begin{array}{l}\text { - Allows facility to change } \\
\text { equipment and processes } \\
\text { and allows new facilities to } \\
\text { be built without air permit } \\
\text { reviews as long as emissions } \\
\text { remain below plantwide limits } \\
\text { - Avoids production delays by } \\
\text { eliminating } 30 \text { to } 50 \text { permit } \\
\text { reviews per year }\end{array}$ & $\begin{array}{l}\text { - Agreement could have } \\
\text { been expedited had public } \\
\text { stakeholders received } \\
\text { education and training on } \\
\text { environmental issues and } \\
\text { the technical aspects of } \\
\text { the industry } \\
\text { - Ongoing technical } \\
\text { assistance for } \\
\text { stakeholders is necessary }\end{array}$ \\
\hline $\begin{array}{l}\text { Lucent } \\
\text { Technologies, } \\
\text { Inc., Facilities in } \\
\text { Allentown and } \\
\text { Reading, PA, } \\
\text { and Orlando, FL }\end{array}$ & $\begin{array}{l}\text { - Consolidated permit } \\
\text { - Participatory approach to goal } \\
\text { setting: Regulators participate } \\
\text { in setting environmental goals } \\
\text { and tracking performance; } \\
\text { environmental organizations, } \\
\text { community groups, employees, } \\
\text { and others provide input }\end{array}$ & $\begin{array}{l}\text { Allows for an annual review } \\
\text { of consolidated, multimedia, } \\
\text { permit rather than multiyear } \\
\text { renewals of individual } \\
\text { permits }\end{array}$ & $\begin{array}{l}\text { - A critical mass of } \\
\text { companies is needed to } \\
\text { complete processes such } \\
\text { as the XL project; they } \\
\text { "jump start" implementa- } \\
\text { tion of long-term } \\
\text { approaches to ensure } \\
\text { sustainability }\end{array}$ \\
\hline $\begin{array}{l}\text { Anderson } \\
\text { Corporation, } \\
\text { Window and } \\
\text { Door Manufact- } \\
\text { uring Plant, } \\
\text { Bayport, MN }\end{array}$ & $\begin{array}{l}\text { Emission rate is tied to } \\
\text { incentives that penalize higher } \\
\text { emissions and reward } \\
\text { reductions in emissions of } \\
\text { volatile organic compounds }\end{array}$ & $\begin{array}{l}\text { - Allows production changes to } \\
\text { be made without obtaining } \\
\text { prior approval }\end{array}$ & $\begin{array}{l}\text { Because the agreement } \\
\text { was signed in June } 1999 \text {, } \\
\text { it was too soon to } \\
\text { determine any lessons } \\
\text { learned }\end{array}$ \\
\hline
\end{tabular}

Source: U.S. Environmental Protection Agency, 1999, Project XL 1999 Comprehensive Report, EPA-100-R-999-008, Office of Administrator, Oct. (EPA 1999i). 